\title{
AN EVALUATION OF LANGUAGE IN BRAIN TUMOUR PATIENTS USING A NEW COGNITIVE-MOTIVATED TESTING PROTOCOL
}

\author{
by
}

Josh Faulkner

\author{
A thesis \\ submitted to the Victoria University of Wellington \\ in fulfillment of the \\ requirements for the degree \\ of Doctor of Philosophy in Psychology
}

Victoria University of Wellington

2015 


\begin{abstract}
In patients undergoing tumour resection surgery, assessment of language is vital, given its crucial role in everyday social functioning. However, despite the unique neuropathological mechanisms in tumours, current literature presents variable results regarding language capabilities in this population. In this thesis we have developed a new neuropsychological test battery, the Brief Language Assessment for Surgical Tumours (BLAST), to specifically evaluate language in brain tumour patients. The BLAST adopts a core skills approach, which identifies and examines 11 core cognitive skills that have been derived based on current cognitive and psycholinguistic theories, and are required for everyday language processing. In this study, we administered the BLAST to a cohort of 40 undifferentiated tumour surgery patients, both pre and postoperatively.
\end{abstract} Also tested were 60 healthy controls categorised into three age groups (18-29, 30-50 and $51+$ years). We examined various aspects of overall test performance in order to evaluate: 1) the overall sensitivity of the test battery at detecting abnormalities in this population; 2) selectivity: the relative incidence of impairments across the various subtests; and 3) their sensitivity to change following surgery. We also explored the effects of lesion localisation and other lesion characteristics (malignancy, oedema and volume) on test performance. Following this, we then used participants' test performance to create operationalised measures of our 11 core cognitive skills, and evaluated these measures in a similar way to the basic test scores. Finally, we used Voxel-Based Lesion Symptom Mapping to determine the specific anatomical predictors for each core cognitive skill score. When investigating overall task performance, we found that $94 \%$ of preoperative patients and $90 \%$ of postoperative patients were impaired in at least one task within the BLAST. Also, $65 \%$ and $68 \%$ of patients had impaired scores on at least one core skill preoperatively and postoperatively respectively. It was also found that the core skills measures 
were effective at discriminating amongst different neurological profiles. Specifically, patients with a left posterior tumour had significantly lower scores than other groups on measures of accessing semantic knowledge, lexical selection and phonological encoding, either pre or postoperatively, or both. Conversely, patients with a left frontal tumour had significantly lower scores on measures of articulatory motor planning and verb retrieval. Our Voxel-LesionSymptom-Mapping analysis corroborated these findings. Lesions within the left superior temporal lobe significantly predicted lows scores in accessing semantic knowledge, lexical selection and phonological encoding. Conversely, lesions within the left inferior, as well as the superior posterior frontal lobe, significantly predicted low scores on goal-driven response selection, articulatory-motor planning and verb retrieval.

We conclude that a core skills approach may be a more effective means of assessing language in tumour populations than more conventional tools that emphasise overall task performance. Such derived measures are sensitive to impairments in this population, and are less likely to be confounded by nonlinguistic impairments that can impact significantly on overall task scores. They may also be useful in guiding postoperative rehabilitation. Further, the scores derived here are associated with quite specific neural substrates, making them potentially useful in guiding surgery and reducing postoperative linguistic deficits. Finally, we conclude that the investigation of tumour populations can also provide unique theoretical insights into language processing and its neural underpinnings in its own right. 


\section{Acknowledgements}

I have been truly privileged to be involved in this research project, and there are a number of people I would like to thank. Firstly, I would like to thank every participant for their generous time and the effort they spent taking part in this research. Thank you in particular to the participants with a brain tumour and their families. I have been so amazed by these patients' willingness to be involved in my research during an extremely difficult time. I have learnt so much from working with this group both at a research, clinical, and personal level.

I would also like to give a special thank you to my primary supervisor Dr Carolyn Wilshire. I cannot thank her enough for her continual support, expertise, knowledge, and sense of humor. Without her, this project would be nonexistent. I am so incredibly grateful to have had the opportunity to work with her, and the skills I have learnt I will use for the rest of my career. I would also like to thank neurosurgeons Mr Andrew Parker and Mr Aliashkevich, neurosurgical administrator Leigh Storm, and Clinical Neuropsychologist Kay Cunningham for their involvement in this project. Further, thank you to my research assistant Bridget Burmester. Thank you to Victoria University for their financial support by a Victoria University $\mathrm{PhD}$ Scholarship (2012-2015) and a travel grant (FSRG 2013), and to Neurological Foundation of New Zealand for their financial support by a Small Project Grant.

Outside of the University, I have been so fortunate to have the support of my family and friends. I would like to thank in particular my wonderful parents, Ian Faulkner and Anita Faulkner, who have supported me throughout my studies. Thank you also to my sister, Francesca Faulkner, for her support and sense of humour. Lastly, I am so thankful to my partner, Chelsea

Leadbetter, for her abundant love and endless support - practically, financially, and emotionally. I could not have gone through this journey without you. 


\section{Contents}

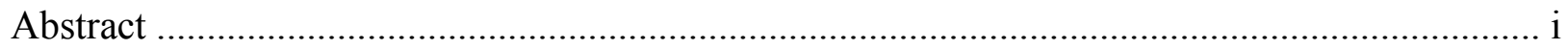

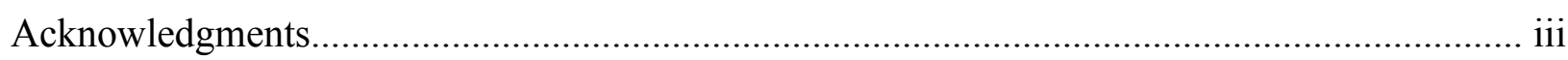

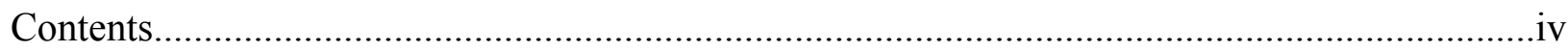

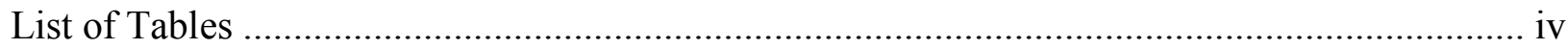

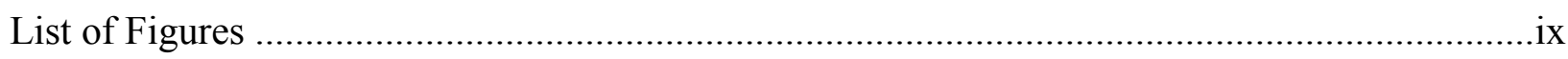

Chapter 1: Introduction and Literature Review............................................................... 1

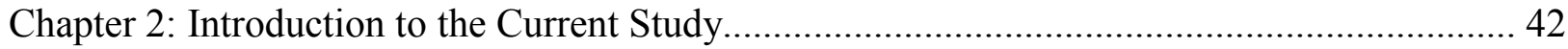

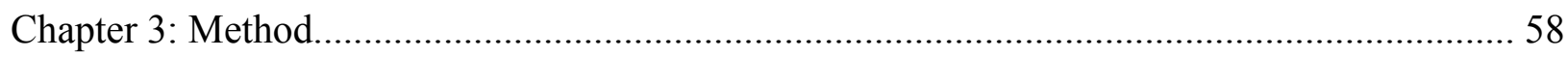

Chapter 4: Overall Task Performance ............................................................................... 90

Chapter 5: Examination of Core Cognitive Skills......................................................... 102

Chapter 6:Voxel-Based Lesion Symptom Mapping Analysis............................................. 126

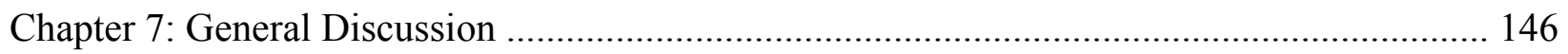

Appendices:

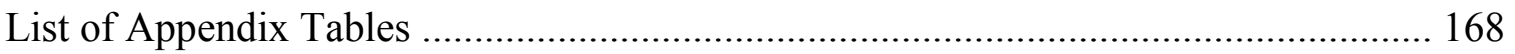

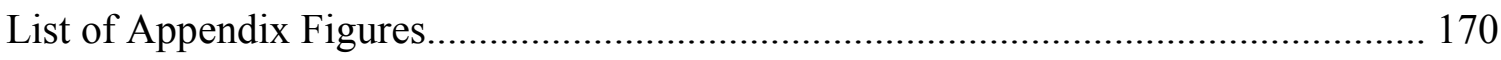

Appendix A: Brain Tumour Patients' Case Descriptions........................................... 172

Appendix B: Information Sheet for Healthy Controls............................................. 194

Appendix C: Neurological Status Questionnaire................................................... 197

Appendix D: Information and Consent Form for Brain Tumour Patients..................... 198

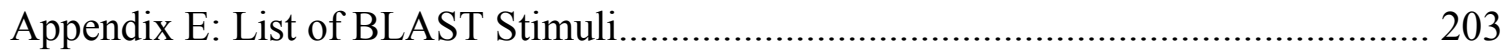

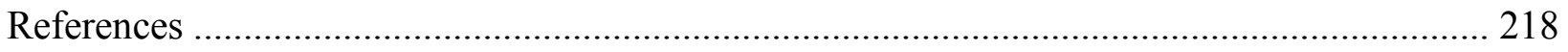




\section{List of Tables}

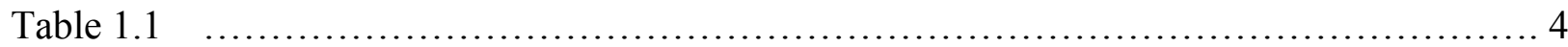

The WHO grading of central nervous system tumours

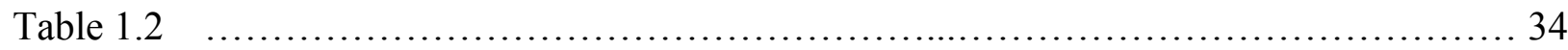

A summary of the core cognitive skills identified from cognitive theories of language and a description of the language profile in patients with a deficit to each skill

Table 1.3

A summary of the cortical regions associated with each cognitive skill assessed by the BLAST

Table 2.1

The eight tests selected for inclusion in BLAST, and where relevant, the variables that were manipulated within each.

Table 2.2

Task profiles used to operationalise the core cognitive skills assessed by the BLAST

Table 2.3 56

Predictions derived for specific brain regions that significantly predict performance in each core cognitive skill.

Table 3.1

The number of patients in each anatomical group pre and postoperatively based on presurgical radiology reports

Table 3.2. 64

The demographic and clinical information of each patient who completed the BLAST

Table 3.3 68

The mean Log CELEX lemma frequencies for the two versions of the Picture Naming Task 
Table 3.4 70

Types of errors coded for in the picture-naming task

Table 3.5

72

The average Selection strength and frequency values for the two versions of the verb generation task

Table 3.6 75

The average frequency and syllable length for the two versions of the picture word verification task

Table 3.7 78

Mean log frequency (Kucera, 1967) and imageability for the four blocks in the first section of the reading test

Table 3.8 85

Key variables manipulated in each of the tests within the protocol, and, where relevant, mean values for these variables

Table 4.1

Tumour patients' overall scores on each task within the BLAST as a percentage both pre and postoperatively

Table 4.2 97

The percentage of patients impaired on each task in the BLAST by tumour type both pre and postoperatively 
Table 4.3

The percentage of patients impaired on each task in the BLAST by tumour oedema presence both pre- and postoperatively

Table 4.4 99

Correlations coefficient calculated for the relationship between tumour volume and overall task performance both pre and postoperatively

Table 5.1 103

Summary of the task profiles associated with each of the 11 core cognitive skills assessed by the BLAST

Table 5.2

Formulae used to calculate each cognitive skill using the key performance measures outlined in table 5.1

Table 5.3

The scores derived for each cognitive skill for each patient who completed the BLAST both preand postoperatively

Table 5.4

The mean T scores for each cognitive skill for high and low malignant tumours, and meningioma's both pre and postoperatively

Table 5.5

Mean T Scores of the core cognitive skills assessed by the BLAST by oedema presence both preand postoperatively 
Correlations between tumour volume and cognitive skill performance both pre and postoperatively

Table 6.1

The percentage of gyri with significant power to detect an effect at the FDR $p<.01$ and FDR $p<$. 05

Table 6.2

The percentage of significant voxels at each significant brain region at the FDR $p<.05$

threshold for preoperative performance

Table 6.3

The percentage of significant voxels at each significant brain region at the FDR $p<.01$ and FDR $p<.05$

Table 6.4

The percentage of gyri with significant voxels at the FDR $P<.05$ threshold for postoperative performance in accessing semantic knowledge, phonological encoding, goal driven response selection and articulatory-motor planning

Table 7.1

Key behavioural and VLSM findings from the anatomical localization analysis of the core cognitive skills both pre- and postoperatively 


\section{List of Figures}

Figure 1.1 The two-stage theory of single word production (adpated from Dell et al., 1997)... 17

Figure 1.2 The four key cognitive skills involved in single word production using picture naming

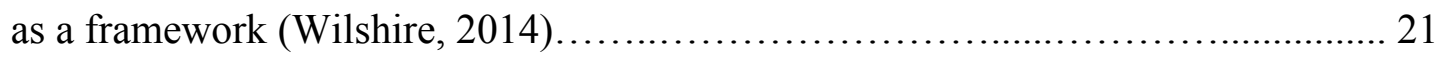

Figure 2.1 The set of 11 core language skills derived from current cognitive theories of language that are assessed in the BLAST.

Figure 3.1 Lesion overlay map for individuals in the left frontal group ..................... 59

Figure 3.2 Lesion overlay map for individuals in the left posterior group................... 60

Figure 3.3 Lesion overlay map for individuals in the right frontal group................... 60

Figure 3.4 Lesion overlay map for individuals in the right posterior group.................... 61

Figure 3.5 Item presentation on a laptop computer for the picture naming task................ 69

Figure 3.6 Item presentation in the verb generation task ...................................... 73

Figure 3.7 Item presentation in the picture word verification task............................. 76

Figure 3.8 Item presentation in the Stroop task......................................... 81

Figure 4.1 The proportion of patients who were significantly different to their appropriate control group based on the number of tasks within BLAST that were completed both pre and postoperative............................................... 93

Figure 4.2 The proportion of patients significantly different to their appropriate control group for each task within BLAST both pre and postoperatively........................... 94

Figure 4.3 The percentage of patients impaired in the most sensitive tasks within BLAST by tumour localization both pre- and postoperatively............................ 96 
Figure 5.1 The numbers of cognitive skills within BLAST that patients were significantly different to their respective controls groups on, both pre and postoperatively..... 111

Figure 5.2 The percentage of patients impaired on each cognitive skills assessed by the BLAST both pre and postoperatively 112

Figure 5.3 The percentage of patients significantly different to there respective control group on each cognitive skill both pre and postoperatively 114

Figure 5.4 The mean T Score for each core cognitive skill for the control group and the right anterior and right posterior group both pre and postoperatively. Error bars represent standard error of the mean.

Figure 5.5 The mean T Score for each core cognitive skill for the control group and the left anterior and left posterior group both pre and postoperatively. Error bars represent

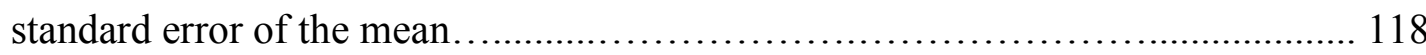

Figure 6.1. Overall preoperative lesion overlap map for brain tumour patients............... 130

Figure 6.2. Power map for preoperative brain tumour patients showing axial slices on a standardised template.

Figure 6.3 VLSM analysis for preoperative performance on accessing semantic knowledge, lexical selection, goal driven response selection, verb retrieval and articulatory motor planning. 134

Figure 6.4 Overall postoperative lesion overlap map for brain tumour patients. 136

Figure 6.5 Power map for postoperative brain tumour patients.

Figure 6.6 VLSM analysis for postoperative performance on accessing semantic knowledge, goal-driven response selection, verb retrieval and articulatory-motor planning.....140 


\section{Chapter 1: Introduction and Literature Review}

Each year, doctors in the United States diagnose approximately 17,000 new primary brain tumours, and 100,000 secondary brain tumours (Porter, McCarthy, Freels, Kim \& Davies, 2010). According to Cancer New Zealand, approximately 100-120 people are diagnosed with a specific malignant brain tumour each year ("Brain Cancer: Glioblastoma multiforme (GBM)," 2013). Over the past few decades, there have been significant improvements in outcomes for patients with brain tumours. The proliferation of more effective treatment procedures, such as the use of new chemotherapy agents and surgical interventions (e.g., awake craniotomy), is increasing life expectancy in this population. With the development of these new techniques, surgeons and clinicians have been able to shift their attention from acute management towards the consideration of quality of life issues, and more specifically how it can be maximised. One important goal in this respect has been the preservation of language function during surgical intervention, since the ability to communicate is such an essential component of everyday life.

Effective language assessment is central to this goal. First, effective preoperative language assessment can identify candidates for awake craniotomy, a surgical technique that has been found to be more effective than standard resective surgery at reducing postoperative language deficits (Ali, Fadel, \& Abouldahab, 2009; De Benedictis, Moritz-Gasser \& Duffau, 2010; Duffau, 2007; Gupta et al., 2007; Peruzzi, Bergese, Viloria, Puente, Abdel-Rasoul \& Chiocca, 2011; Sacko et al., 2011). Second, preoperative assessment can also help assist in the selection of language tasks for intraoperative testing during awake craniotomy. This can help the neurosurgeon identify cortical tissue that is essential to language for each patient, and thereby help preserve language function (De Witte \& Mariën, 2013). Third, the detection of language impairments by postoperative assessment can guide more effective rehabilitation (Davie, 
Hutcheson, Barringer, Weinberg, \& Lewin, 2009). However, before these goals can be met, it is imperative that we examine in detail the language capabilities of brain tumour patients generally. This thesis aims to carry out such an endeavour. The following section will give a brief overview of the characteristics and etiologies of a brain tumour. Following this, we then explore the current literature pertaining to the implications of a brain tumour on language functioning.

\section{Brain Tumours: A Brief Overview}

A brain tumour is a solid abnormal mass of tissue within the brain or the central spinal canal. It comes in various shapes, locations and sizes, and exhibits many different types of growth patterns (Ricard, Idbaih, Ducray, Lahutte, Hoang-Xuan, \& Delattre, 2012). Brain tumours are created by abnormal and uncontrolled cell division. They can develop within the brain itself (e.g., glial cells: astrocytomas, oligodendroglioma, ependymomas), but also in lymphatic tissue (e.g., cerebral lymphoma), in blood vessels (e.g., hemangioblastoma), in the cranial nerves (e.g., schwannoma), in the brain meninges (e.g., meningioma), the skull (e.g., chondrosarcomas pituitary gland (e.g., pituitary adenoma), or pineal gland (e.g., pineocytoma). Tumours that grow in this manner are called primary brain tumours. The most common type of primary brain tumours is gliomas $(50.4 \%)$, followed by meningiomas $(20.8 \%)$ and then pituitary adenomas (15\%) (Park, Kim, Sade \& Lee, 2009). Brain tumours may also spread from cancers primarily located outside the central nervous system; these are called secondary or metastatic tumours. The most common source of origin for metastatic tumours derives from carcinomas of the breast, lung, and malignant melanoma. Metastatic tumours occur more frequently than primary brain tumours (4:1) (Marsh, 2009).

Currently, no one knows exactly what causes brain tumours, and there have only been a few risk factors identified. For example, children who receive radiation to the head have a higher 
risk of later developing a brain tumour (for review see Pettorini, Park, Caldarelli, \& Massimi, 2008), as do people who have rare genetic conditions (Behin, Hoang-Xuan, Carpentier, Delattre, 2003). However these cases only represent a fraction of those who are diagnosed with a primary brain tumour each year.

Brain tumours can result in a range of neurological symptoms, and these can be divided into three main categories. The first set of symptoms derives from increased intracranial pressure. Clinically, this translates into headaches, vomiting, altered state of consciousness, dilation of the pupil, and papilledema (swelling of the optic disc, located at the back of the eye). The second results directly from damage to the brain by either compression or infiltration of the tumour. Any type of focal neurological symptom may occur, such as motor, cognitive, and behavioural impairment, and/or personality or emotional changes. The third and final category is irritation. This includes such symptoms as abnormal fatigue, weariness, absences, and tremors (see especially Arber, Faithfull, Plaskota, Lucas \& de Vries, 2010; Cahill, LoBiondo-Wood, Bergstrom, \& Armstrong, 2012; Davies \& Clarke, 2004; Forsyth \& Posner, 1993; Gutin \& Posner 2000; Omuro, Leite, Mokhtari \& Delattre, 2006; Squires, 1989;). Importantly, this also includes epileptic seizures, which are the most common neurological symptom that will motivate a brain tumour patient to seek medical attention. For example, Taphoorn and Klein (2004) reported that $80 \%$ of brain tumour patients had a seizure prior to diagnosis.

The neuroanatomical locations, as well as the rate of growth and invasiveness of a brain tumour, are key determiners of this symptomology (DeAngelis, 2001). Tumour growth and invasiveness are directly related to the histological features of a tumour (Bosman, Carneiro, Hruban \& Theise, 2010). Broadly speaking, a tumour can either be cancerous (malignant) or non-cancerous (benign). More specifically, the World Health Organization (WHO) has 
developed a malignancy scale, from grade I-IV, to quantify the histological features of brain tumours (Kleihues \& Sobin, 2000). The specific histologic features used for each grade are presented in Table 1.1. Patients with a high-grade brain tumour have a much poorer prognosis than lower grade tumours. A high-grade tumour carries a prognosis of 6-18 months, depending on age and disability at presentation. A low-grade brain tumour can carry a prognosis of many years, but will ultimately transform to a higher grade if there is incomplete surgical resection of the tumour (Marsh, 2009).

Table 1.1

The WHO grading of Central Nervous System tumours

WHO grade Lesions with low proliferative potential, a frequently discrete nature, and

I the possibility of cure following surgical resection alone

WHO grade Lesions that are generally infiltrating and low in mitotic activity but

II recur

WHO grade Lesions with histologic evidence of malignancy, generally in the form of

III mitotic activity, clearly expressed infiltrative capabilities, and anaplasia

WHO grade Lesions that are mitotically active, necrosis-prone, and generally

IV associated with rapid preoperative and postoperative evolution of disease.

\section{Brain Tumours and Language}

There is consensus in the literature that a brain tumour can have a profound impact on cognitive functioning (see especially Klein et al., 2002; Meyers, Hess, Yung \& Levin, 2000; Murray et al., 2000; Scheibel, Meyers \& Levin, 1990; Talacchi, Santini, Savazzi \& Gerosa, 2011; Taphoorn \& Klein, 2004; Taylor et al., 1998). One such aspect of cognitive functioning 
within this domain is language. It has been difficult to establish the exact prevalence of linguistic dysfunction in this population, and this is due to a number of pitfalls evident throughout the literature. Specifically, these research investigations that have set out to investigate language dysfunction in brain tumour patients differ greatly as to the characteristics of the patient sample, and type of surgical intervention used. Such studies investigate language function in awake craniotomy patients only, a procedure that may be especially indicated for when a tumour is in "classical" language areas (e.g., Broca's and Wernicke's area). Language dysfunction is likely to be more prevalent in this kind of sample than in an undifferentiated sample. Others investigate language function only in left hemisphere patients, and often only in cases where the tumour is in close proximity to the traditional language areas. Consequently, it is difficult to draw any definitive conclusions across these investigations, and very little is therefore known about language capability in tumour patients more generally.

Furthermore, there is huge variation in the testing protocols used throughout these investigations. Measures used range from informal self-report measures (e.g., Thomas et al., 1995) to formal aphasia assessments (e.g., Duffau, Peggy Gatignol, Mandonnet, Capelle \& Taillandier, 2008; Whittle et al. 1998) through to specific neuropsychological protocols (e.g., Bello et al., 2007; Sanai, Mirzadeh, \& Berger, 2008). Caution must therefore be used in interpreting linguistic deficit rates in this population, and one must always consider the sample of brain tumour patients used, as well as the sensitivity of the linguistic assessment methodology.

With this in mind, from the growing body of research exploring linguistic deficits in brain tumour patients, it is clear that language dysfunction occurs in this population. In preoperative samples, estimates of the prevalence of language deficits range from $37 \%$ to $63 \%$ (Bello et al., 2007; Haglund, Berger, Shamseldin, Lettich, \& Ojemann, 1994; Recht, McCarthy, 
O’Donnell, Cohen \& Drachmann, 1989; Sanai, Mirzadeh, \& Berger, 2008; Tandon \& Mahapatra, 1993; Thomas, O’Connor \& Ashley, 1995; Whittle, Pringle \& Taylor, 1998;). For example, Thomas, O'Connor and Ashley (1995) found that in an undifferentiated sample of 116 patients with a high-grade glioma, 37\% had a "speech deficit" at presentation, according to the report of the patients themselves and/or their primary caregivers. Whittle, Pringle and Taylor (1998) found that in 40 left hemisphere brain tumour patients about to undergo tumour resection surgery, 62.5\% were classified as dysphasic according to the Western Aphasic Battery (WAB) (Kertesz, 1982). Finally, Sanai, Mirzadeh, and Berger (2008) also found that of 250 left hemisphere glioma patients, $36.4 \%$ had a language deficit preoperatively. This was identified by impairment on at least one of the following tasks: counting, object naming, single word reading, sentence repetition, and writing words and sentences.

These patterns of linguistic dysfunction occur as a result of the infiltration, displacement, and compression of both gray and white matter (subcortical nuclei) within the cerebral cortex. Furthermore, it is well know that language abilities rely on the integrity of neural networks whose cortical nodes are frequently located within more than one lobe of the left hemisphere (sometimes both hemispheres) and are connected by white matter pathways (for review see Friederici, 2014). A brain tumour can also impact on language by causing disruption in the connectivity of white matter pathways due to deviation, infiltration, edematous and destruction (Jellison, Field, Medow, Lazar, Salamat \& Alexander, 2004).

Second, language dysfunction in brain tumour patients may occur as a result of the surgery itself. This may occur due to the resection of brain tissue essential for language function (known as eloquent cortex), or from postoperative complications such as swelling and inflammation (Heimans \& Reijneveld, 2012). Discrepancies in the literature again have made it 
difficult to draw conclusive evidence about the effects of neurosurgery on language processing.

Some research has found a low incidence of language deficits caused by surgery in postoperative brain tumour patients. For example, Duffau and colleagues (2008) assessed a series of 115 left hemisphere patients with grade II gliomas on the Boston Diagnostic Aphasia Examination (Goodglass, Kaplan \& Barresi, 2001) both before and after awake craniotomy surgery, and observed new language impairments following surgery in only $2 \%$ of patients (Duffau, Peggy Gatignol, Mandonnet, Capelle, \& Taillandier, 2008). McGirt and colleagues (2009) conducted a comprehensive retrospective analysis of 306 undifferentiated patients who had undergone resection of glioblastoma. They found that 5\% of patients developed an acquired language deficit postoperatively (the language assessment protocol used in this investigation was not explicitly stated) (McGirt, Mukherjee, Chaichana, Than, Weingart, \& Quinones-Hinojosa, 2009).

However, several other studies have suggested that language impairments induced as a result of surgery may be more common. For example, Bello and colleagues (2007) conducted a comprehensive language examination in 88 left hemisphere glioma patients who had undergone intraoperative mapping of subcortical language tracts during an awake craniotomy. The participants performed a range of language tasks, including picture naming, famous face naming, action naming, word repetition (real and nonsense words), picture-word matching, and letter/category fluency (e.g., "name as many items as you can think of that start with letter 'F'/ belong to the category 'animals'). In patients whose intraoperative mapping revealed positive subcortical language sites $(\mathrm{N}=52)$, evaluation of language three days after surgery showed new deficits or worsening of existing language deficits in $67.3 \%$ of patients (Bello et al., 2007). In addition, Ilmberger and colleagues (2008) conducted a prospective longitudinal study to evaluate language in 149 patients with a tumour in close proximity to or within language areas (therefore 
the location of the tumour was confined to the left hemisphere). To achieve this, the Aachen Aphasia Test (AAT: Huber, Poeck, \& Willmes, 1983) was used. This standardized battery consists of five subtests: 1) the token test, which involved pointing to and manipulating geometric forms in response to a command; 2) repetition of phonemes, words and sentences; 3) written language, consisting of reading and writing single words; 4) naming, involving naming visually presented objects, colours and scenes; and 5) comprehension, using the picture word matching task. Patients were classified as having a language deficit whenever they showed at least mild disturbance in one of the subtests above. Using this classification, it was found that within 21 days after surgery $32 \%$ of patients without preoperative deficits had a new language deficit (Ilmberger, Ruge, Kreth, Briegel, Reulen, \& Tonn, 2008). Thus, brain tumour patients maybe particularly susceptible to new or worsening of existing language function shortly postsurgery (for a review of similar findings see Finch \& Copland, 2014).

Within this body of literature, a limited pool of research investigations have suggested that the linguistic profiles of brain tumour patients are distinctly different from those observed in post-stroke aphasia. First, it has been found that language impairments in brain tumour patients are more likely to appear as mild deficits in common aphasic testing protocols. In contrast, language impairments evoked by a stroke are more likely to be severe and appear as more globalised deficits in common aphasic testing. For example, Anderson, Damasio and Tranel (1990) compared a sample of 17 brain tumour participants (eight with a left hemisphere tumour, nine with a right hemisphere) with an equal sized sample of unilateral stroke patients. Each stroke patient was anatomically matched to one of the tumour patients on the basis of lesion location and size. It was specified that lesions in stroke cases could be either as large or smaller than the lesion in the matched tumour cases. The Multilingual Aphasia Battery (Benton, 1969) 
was administered as well as the Boston Diagnostic Aphasia Examination Reading Sentences and Paragraphs subtest (Goodglass \& Kaplan, 1983). Despite the close matching of the two groups, there were major differences in each group's performance. Of the left hemisphere cases, all of those in the stroke group had more severe language deficits than did those in the tumour group, despite the fact that the average lesion size was larger in the brain tumour group. The left hemisphere stroke subjects showed greater impairment than the tumour group in all subtests of the Multilingual Aphasia Examination (Benton, 1969), as well as on the Boston Aphasia Examination Reading subtest. Furthermore, Davie, Hutcheson, Barringer, Weinberg, and Lewin (2009) used the Western Aphasia Battery (Kertesz, 1982) to evaluate the language performance of 65 patients who had recently undergone malignant tumour resection. They found that anomic aphasia was the most common type of aphasia in this group (48\% of patients), whereas global aphasia was the least common (3\% of patients). This markedly contrasts with the profile of aphasia in stroke patients, where there are higher rates of global aphasia (20-40\%) and lower rates of anomic aphasia (9-28\%) (for similar findings of aphasia profile in stroke, see Kauhanen et al., 2000; Kertesz \& Sheppard, 1981; Pashek \& Holland, 1988; Pedersen et al., 2004).

There also appears to be differences in the patterns of language recovery observed between brain tumour patients postoperatively and in post-stroke aphasia (Shafi \& Carozza, 2012). In post-stroke aphasia, some degree of recovery typically occurs spontaneously within eight to 12 weeks, and peaks after one year with only minimal improvements thereafter (see Berthier, 2005). In brain tumour patients, studies have shown that the majority of patients who experience a decline in language function immediately post surgery, will experience considerable recovery of function within three months of surgery (Finch \& Copland, 2014; Wu et al., 2011). This pattern has been attributed to a number of factors that are more salient in brain tumour 
compared to stroke. These include resolution of postsurgical oedema, transient retraction injury, initial displacement of neural structures, and neuroplastic mechanisms (Bello et al., 2007). However, although such a pattern has been argued within the literature, it must be noted here that some studies do present a far less promising picture of language recovery in this population. For example, Papagno, Casarotti, Comi, Gallucci, Riva, and Bello, (2012) found that at three months post surgery, a significant proportion of left hemisphere brain tumour patients were still impaired on certain language tasks. Specifically, $48 \%$ of left temporal patients who had a low-grade glioma were impaired in a naming famous people task ${ }^{1}$, and $40 \%$ of left frontal patients were impaired in a letter fluency task. In addition, Ilmberger et al., (2008) used a battery of language tasks previously described and found that in a sample of 153 awake craniotomy patients, 17.6\% of patients had a persistent postoperative language disturbance seven months post surgery.

The emergence of evidence that indicates that language profiles in tumour patients are different to those in stroke patients is perhaps not surprising, given the contrasting pathological mechanisms between the two neurological entities. One such difference is that unlike stroke, which generally has an acute onset, tumour growth progresses gradually, allowing for the possibility of cortical reorganisation (for discussion see Miceli, Capasso, Monti, Santini \& Talacchi, 2012). Indeed, there is evidence that Broca's aphasia, which is commonly observed in post-stroke aphasia following damage to Broca's area and surrounding regions, is rarely observed when a tumour develops in that region (Plaza, Gatignol, Leroy \& Duffau, 2009). This observation might well be attributable to the greater opportunities for neuroplasticity phenomena to occur in tumour patients (Duffau, 2007). A second difference is that whereas vascular damage results directly in neural cell necrosis, tumours grow by infiltrating nonneural cells (e.g., glial

\footnotetext{
${ }^{1}$ The famous person to be named belonged to one of four professional categories (artists/scientist, athletes, actors, politicians) and are graded for the period of his/her fame.
} 
cells or meningeal tissue), and only begin to impact on neural function when there is significant displacement and compression of neural tissue. For that reason, tumour growth may be considerably advanced before any functional impairment is observed (Miceli et al., 2012). A third difference between vascular and tumour damage concerns the distribution of the lesion. The cerebral regions most vulnerable to stroke (particularly ischemia) are those that lie within the region supplied by the occluded artery/arteriole, and consequently some regions are consistently more vulnerable than others. The cerebral regions impacted by a tumour, on the other hand, can be extremely variable, particularly when the tumour involves interstitial tissue.

Therefore, it is safe to say that the language profiles observed in subjects with brain tumours are likely to differ significantly and substantially from those observed in patients with aphasia of vascular origin. Consequently, classic test batteries (e.g. the Boston Diagnostic Aphasia Examination (BDAE) (Goodglass, Kaplan \& Barresi, 2001), or the Western Aphasia Battery (WAB) (Kertesz, 1982)) that have been developed primarily for the classification of "classical" aphasic syndromes induced by a cerebrovascular accident (Broca's, Wernicke's, conduction, transcortical etc.), may not be optimal for the detection of language deficits in brain tumour patients. Indeed, this is a view that has been espoused previously by a number of researchers (De Witte \& Mariën, 2013; Meyers \& Brown, 2006; Miceli et al. 2012; Påhlson, Ek, Ahlström \& Smits, 2003; Talacchi, Santini, Savazzi \& Gerosa, 2010).

One recent study serves as a rare example of a neuropsychological assessment tailored specifically to tumour patients. The recent Milano-Biocca Battery is designed to investigate the performance of tumour patients in three cognitive domains: language, memory and executive function (Papagno, Casarotti, Comi, Gallucci, Riva, \& Bello, 2012). The entire test battery has been administered to 226 tumour patients both pre- and postoperatively, and at three months post 
surgery. To investigate language, the following tasks were administered: letter and category fluency, naming famous persons from photographs, object naming, picture-word matching, action naming, naming by description, and real word, nonword and sentence repetition. Although only preliminary results have been published, Papagno and colleagues (2012) found that the following language tasks were the most sensitive at detecting language impairments before surgery and at three-month follow up: naming famous people, action naming, object naming and category and letter fluency. In addition, patients were categorised into four groups based on the anatomical location of the tumour - left frontal, left temporal, right frontal, and right temporal. It was found that tumour localisation was a strong predictor of performance in most of the language tasks administered. Specifically, patients in the left temporal group performed more poorly than all other groups in naming people and objects, and this was true of all the testing points. They were also worse in category fluency at three months post surgery. In contrast, patients with a left frontal lesion performed more poorly than all other groups in the letter fluency task, and this was true both preoperatively and at three months post surgery (Papagno et al., 2012).

However, comprehensive neuropsychological batteries of this kind take time to administer (up to two hours for the Milano-Bicocca), and even then, the number of test results that are directly pertinent to language function is relatively small. Here we focus on just one cognitive domain - language. This will enable us to test that domain more extensively, and in doing so, perhaps identify those measures that are most sensitive at detecting impairment in this population. It is interesting to note that in the original study of the Milano-Biocca Battery, only five of the 11 language tasks in the original battery were sufficiently sensitive at detecting impairment to be considered worthy of reporting by the authors (Papagno et al., 2012). A broader, 
more exploratory assessment of language, which aims to assess as many aspects of language function as possible, as sensitively as possible, may be a useful first step in the development of shorter, more carefully tailored assessment protocols for use with a brain tumour population.

Current cognitive theories of language may provide a particularly useful starting point for such an endeavour, because such theories delineate the various core cognitive operations that are essential for performing particular language behaviours. Assessments can then be developed which target each of these "core skills". This approach offers a systematic, theory-driven method for deciding what tasks should be included in the assessment. Also, if applied effectively, this method may help to maximise the range of skills examined (thereby providing better breadth and sensitivity), and is likely to offer greater power to discriminate amongst different language profiles based on the neurological profile of the patient. The following section reviews cognitive and neuropsychological research that is relevant to this objective. It is then followed by a review of research that investigates the neural structures associated with each core skill, with a particular emphasis on lesion studies.

\section{Cognitive Theories of Language}

Current cognitive theories of language posit that key language behaviours, such as producing words and sentences, understanding spoken words and sentences, and reading, can be decomposed into several more elementary cognitive skills. Indeed, impairments to each cognitive skill have been found to be associated with a unique neurological and linguistic profile (see Table 1.2 for a summary of these skills and their associated neurological profiles). Before beginning this review, we acknowledge that within these models there is considerable debate in the literature as to the exact cognitive mechanisms associated with certain skills. These debates go beyond the aims of this investigation. We have therefore chosen to examine only those 
cognitive skills for where there is widespread agreement for their existence in the literature.

\section{Accessing Semantic Knowledge}

If we begin by considering single word production, most psycholinguistic theories start with the simple task of naming a pictured object. There is wide agreement in the literature that at least four key cognitive skills are required for this task (see Figure 1.2). The first cognitive skill, which we will call accessing semantic knowledge, refers to the process of retrieving information about the semantic category, function, colour, size, etc., of the item to be named (Friedmann, Biran \& Dotan, 2013). Many psycholinguistic models suggest that this semantic information is organised into a network consisting of an interconnected matrix of nodes, which correspond to individual features of the target item. For example, in the case of DOG, the attributes has four legs, barks, and chews bones, might all be coded for by different, but interconnected nodes, which collectively form the semantic representation for dog (e.g., Masson, 1991, 1995). Once partial information about an item is activated, activation spreads to these interconnected nodes, making additional information about the item accessible (Collins \& Loftus, 1975; Neely 1997).

Evidence to suggest that accessing semantic knowledge is a distinct entity can be drawn from the neuropsychological literature. In a neurodegenerative disorder known as semantic dementia (SD), the most prominent early feature and presenting complaint is a difficulty in "remembering" the names of people, places and things (e.g., Pijnenburg, Gillissen, Jonker, Scheltens, 2004; Thompson, Patterson, \& Hodges, 2003). Spontaneous speech retains its normal grammatical structure, but there may be frequent pauses as the speaker struggles to find a particular word, and some terms may be replaced by commoner, more general terms (e.g., "thing" instead of "kettle", and "doing" instead of "cooking"). Pronunciation and phonological skills are usually unaffected (Adlam et al., 2006; Ash, Moore, Antani, McCawley, Work, \& Grossman, 
2006). As the disease progresses, difficulties become evident in tasks involving comprehension. For example, when asked to define a word, people with SD may be able to provide only very general information (e.g., "Ostrich. Can you say that?" "Yeah, ostrich"; "What is a hippopotamus?" "An animal”) or simply absent ("I think I've heard of a hippopotamus, but I can't say what it is") (Hodges \& Patterson, 2007).

Tasks that are commonly used to examine SD are picture naming, category fluency and picture-word matching. On picture naming, individuals with SD may be particularly prone to semantic errors, where the target word is replaced by another word from the same semantic category (e.g., zebra -> "giraffe") (Garrard, Perry \& Hodges, 1997; Hodges, Graham \& Patterson, 1995; Jefferies \& Lambon Ralph, 2006). On category fluency tasks, there is also a notable decrease in the number of words that the individuals can generate when given a semantic category; in contrast, letter fluency is relatively spared (Bozeat, Lambon Ralph, Patterson, Garrard \& Hodges, 2000; Graham \& Hodges, 1997; Hodges, Patterson, Oxbury \& Funnell, 1992; Rascovsky, Salmon, Hansen, Thai, \& Galasko, 2007;). This is an unusual pattern, in most braindamaged patients, letter fluency is disproportionately impaired. Finally, on picture word matching tasks, SD patients tend to show confusion between semantically related items. They are particularly prone to errors on tasks where they must choose a picture match for a word from amongst a number of alternatives from the same semantic category (see esp. Corbett, Jefferies, Ehsan \& Lambon Ralph, 2009).

\section{Lexical Selection}

Many theories of single word production also distinguish this general semantic processing stage from a subsequent lexical (or lemma) selection stage. This cognitive skill involves selecting the appropriate word from the mental lexicon that best matches the semantic 
concept in mind. In many theories, this stage of processing in conceptualised within a spreading activation framework (e.g., Caramazza 1997; Dell, 1986; Levelt, 1999, Rapp \& Goldrick, 2000; Roelofs, 2004; Ruml, Caramazza, Capasso \& Miceli, 2005; Schwartz, Dell, Martin, Gahl \& Sobel, 2006). For example, according to Dell's (1986) model, depicted in Figure 1.1, during single word production, semantic nodes that represent aspects of the meaning of an item transmit activation to their associated lexical units. All units of associated words receive some activation (for example, if the target item is a cat, the lexical unit for "dog" will also become activated, because dogs possesses some of the same semantic attributes). However, the word that contains the greatest number of semantic features will generally receive the most activation. The lexical selection step is complete when the most highly activated lexical unit is "selected" for production.

Support for the existence of this lexical selection process, as distinct from semantic access, comes from studies that have demonstrated reduced naming efficiency in normal speakers when two or more words "compete" for selection. For example, in the picture-word interference task, participants must name a picture, which is accompanied by an irrelevant auditory or written word (for example, a picture of a tiger is accompanied by the word "lion"). Specifically, when the irrelevant distractor word is semantically related to the target, and is presented just before or at the same time as the picture, individuals are substantially slower to name the picture than they are when the word is unrelated (Glaser \& Düngelhoff, 1984; Roelofs, 1992; Starreveld \& La Heij, 1995, 1996). It has been suggested that this delay is caused by competition for selection between the two concurrently activated lexical items - that is, the name of the target item, and the distractor word itself. 


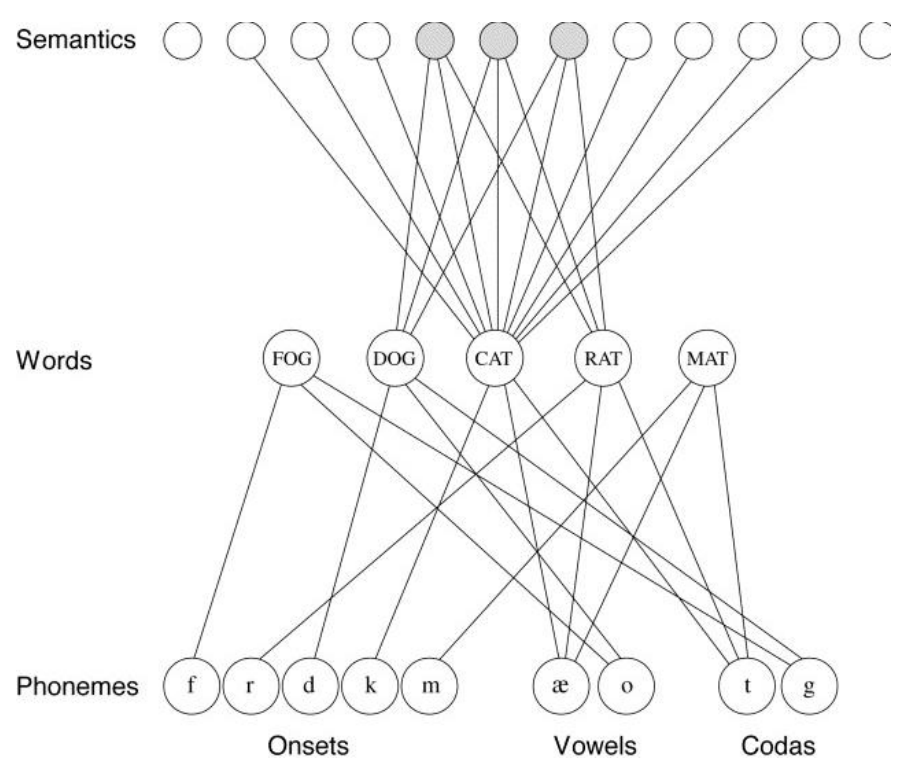

Figure 1.1: The two-stage theory of single word production (adpated from Dell et al. 1997).

One neuropsychological profile in which this lexical selection stage appears to be implicated is classical or pure anomia (Andreetta, Cantagallo \& Marini, 2012; Butterworth, 1992; Lambon Ralph, Sage \& Roberts, 2000; McNeil, Odell, \& Tseng, 1991). The hallmark feature of this disorder is poor picture naming without any accompanying impairment in general semantic knowledge (for example, comprehension may be normal; Franklin, Howard \& Patterson, 1995; Howard, 1995; Gonon, Bruckert \& Michel, 1989; Laine, Kujala, Niemi, \& Uusipaikka, 1992; Raymer et al., 1997). In picture naming tasks, these patients tend to produce a mix of errors, including many failures to respond and/or circumlocutions ("They live in the sea and they lay eggs on the beach, but I can't think of the name"). It has been argued that these kinds of errors in particular may be a consequence of a failure to retrieve any lexical item from the mental lexicon (for review, see Dell, Lawler, Harris \& Gordon, 2004). For example, case, $\mathrm{RBO}$, who suffered from a ruptured A-V malformation of the left posterior communicating artery, failed to provide any response at all to $40 \%$ of the items in a large picture naming test (Miceli, Amitrano, Capasso \& Caramazza, 1996). Individuals with this profile may also produce 
semantic errors, but in contrast to individuals with SD, they are more likely to recognise their errors as incorrect (Lambon Ralph, Sage \& Roberts, 2000). One final hallmark of individuals with this profile is a marked word frequency effect in naming and similar word production tasks: individuals are considerably more prone to errors/omissions on lower frequency words (e.g., case AW: Jacobs, Singer \& Miozzo, 2004; case FR: Avila, Lambon Ralph, Parcet, Geffner \& Gonzalez-Darder, 2001). It has been suggested that the lexical representations of high-frequency words have higher resting levels of activation or lower selection thresholds than those of lower frequency words. Therefore, they require less activation to reach the activation level that is critical for selection, so may be less affected by any impairment affecting lexical activation (for review see Nickels, 2002).

\section{Phonological Encoding}

Subsequent to lexical selection, cognitive models of single word production generally propose a stage of processing called phonological encoding. This involves retrieving information about the selected word's sound form from the mental lexicon (see especially Dell, 1986; Levelt, 1999; Rapp \& Goldrick, 2000; Schwartz et al., 2006). This abstract sound information then forms the primary input for articulatory-motor programming. Some models suggest that the phonological encoding process can itself be subdivided into a number of smaller cognitive processes. For example, Levelt (1999) proposed that the relevant phonological segments and their metrical information (for example, the number of syllables and their stress pattern) are retrieved independently and in parallel, and then subsequently combined.

There is a wealth of evidence for the existence of an aphasic disorder that arises due to a selective impairment in the ability to encode phonological information. In conduction aphasia individuals speak fluently and with ease, but produce a number of phonological errors in 
spontaneous speech and on a range of single word production tasks (for example, the person may say "pabacco" instead of "tobacco"; see Buchsbaum, et al., 2011). As many as 50\% of these individuals' responses may be phonological errors (Kohn \& Goodglass, 1985; see also Butterworth, 1992; Caplan, Vanier \& Baker, 1986; Kohn \& Smith, 1993; Pate, Saffran \& Martin, 1987; Pradat-Diehl, Tessier, Vallat, Mailhan, Mazevet \& Lauriot-Prevost, 2001; Wilshire \& McCarthy, 1996). These patients also exhibit a number of other features consistent with a deficit in phonological encoding. For example, they tend show a strong length effect in picture naming - that is, they are less accurate at producing words that contain multiple syllables (Caplan, Vanier, \& Baker, 1986; Kohn \& Smith, 1993; Pate, Saffran, \& Martin 1987; Wilshire \& McCarthy, 1996; Wilshire, 2002). Most models predict that multisyllabic items will place extra demands on phonological encoding, as additional phonemes need to be retrieved and/or inserted into the correct metric frame. In addition, they may also produce phonological errors in auditory word repetition tasks, particularly on longer words (see esp. Caplan, Vanier \& Baker, 1986; Caramazza, Basil, Koller \& Berndt, 1981; Dell, Schwartz, Martin \& Saffran 1997; Strub \& Gardner, 1974). One of the critical prerequisites for successful performance in this task is likely to be the ability to encode the phonological information of the word to be repeated.

\section{Articulatory-Motor Programming}

According to most theories, the output of the phonological encoding process consists of fairly abstract, syllabified phonological words that are then translated into articulatory-motor programmes. This final processing stage will be referred to as articulatory-motor programming. This process involves constructing a motor plan for the articulatory execution of that utterance (see esp. Romani, Olson, Semenza, Granà, 2002; Romani \& Galluzzi, 2005; Indefrey \& Levelt, 2004). According to Levelt's (1999) theory, speakers have access to a repository of syllabic 
gestures, termed the 'mental syllabary', that contains the articulatory scores for at least the most common syllables in language (Levelt 1992; Levelt \& Wheeldon, 1994). As soon as a syllable emerges from the phonological encoding process, the corresponding syllabic articulatory gesture will be selected from the repository (Indefrey \& Levelt, 2004; see also Dronkers, 1996; Kerzel \& Bekkering, 2000).

There is certainly supporting neuropsychological evidence for the existence of a distinct articulatory-motor programming stage of processing. Apraxia of speech (AOS) has been described as a disorder of motor-speech programming, which leads to errors in sequencing, timing, coordination, initiation and vocal tract shaping (Darley, Aronson, \& Brown, 1975; Kent \& Rosenbek, 1983). Hallmark features of patients with AOS are articulatory errors and prosodic abnormalities. Articulatory errors are more common on certain kinds of segments than others for example, affricates (e.g., $c h$ and $j$ ) and fricatives (e.g., $s$ and $z$ ) tend to be particularly errorprone, and errors are also more common on consonant clusters rather than singleton consonants (e.g. 'strict' will be more difficult than sit) (for review see Ogar, Slama, Dronkers, Amici, \& Gorno-Tempini, 2005). Patients with AOS additionally have a markedly reduced rate of speech considerably lower than that of individuals with aphasia that do not have AOS (Canter, Trost \& Burns, 1985). 


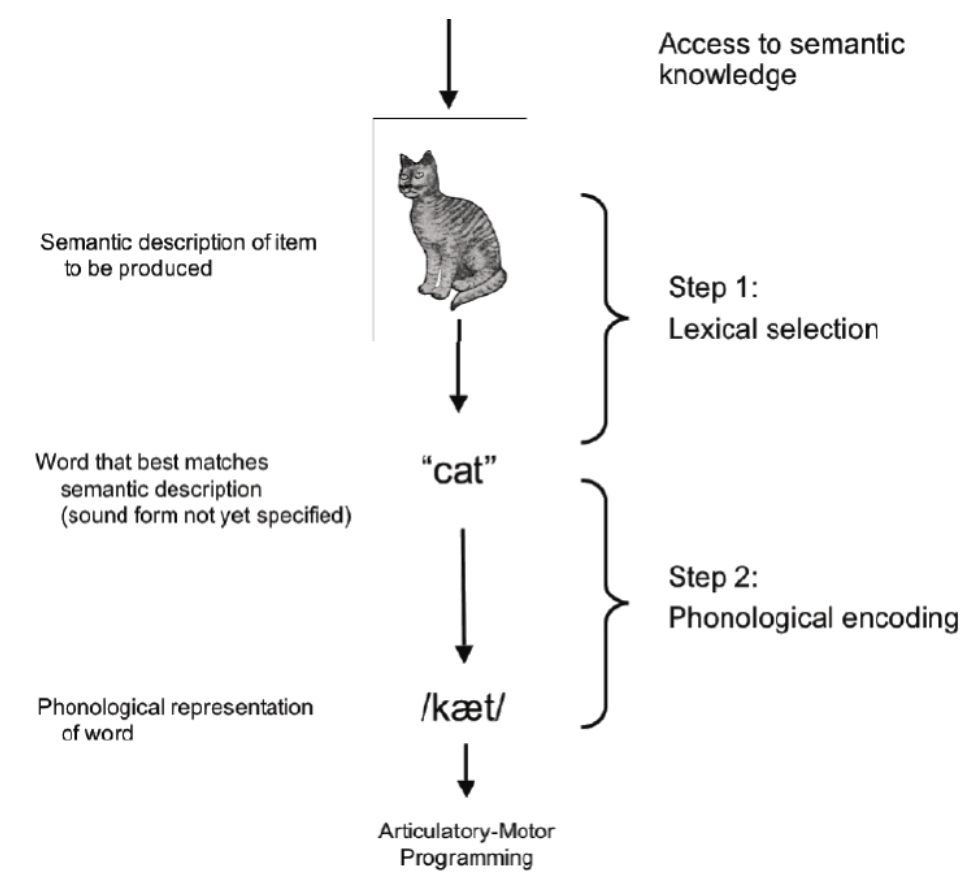

Figure 1.2: The four key cognitive skills involved in single word production using picture naming as a framework (adapted from Wilshire, 2014)

\section{Sentence-level Planning}

So far, we have considered the production of single words, but within language a crucial skill is being able to incorporate these words into a grammatically correct sentence. Cognitive theories of sentence-level production vary considerably in the specific cognitive processes they propose (see especially Dell, 1986; Garrett, 1975; Levelt, 1989; Levelt, 1999; Stemberger, 1985). Some theorists propose a frame-allocation process. That is, the speaker builds an abstract representation of a "sentence "frame" which specifies the classes of lexical elements that appear, their order, and any necessary grammatical elements. In most models the elements in the frame are defined grammatically (noun, verb, etc.). Words that fulfil these grammatical criteria can then be inserted, resulting in a fully formed ordered 'plan' of the sentence (Garret, 1975, 1976, 1982). In some models, selection of the appropriate verb is crucial for the development of an appropriate sentence frame, as the verb specifies important aspects of the frame, such as the 
number of arguments (how many direct and indirect objects) the verb can take (e.g. Ahrens, 2003; Levelt 1989, 1999; Shapiro \& Levine, 1990, Shapiro, Zurif, \& Grimshaw, 1987; Trueswell \& Kim, 1998). A rather different view recently espoused is that the sentence "plan" might be more like a proposition - that specifies the main entities, their properties and their relations to, or actions upon one another (e.g. dog-> agent, cat-> patient chase-> action; Chang, Dell and Bock, (2006)). The most salient conceptual element in this proposition wins the competition to initiate sentence planning. Sentence order and grammatical structure is then generated by applying a set of rote-learned ordering rules. In sum, a number of cognitive skills have been identified that are uniquely involved in sentence-level planning. These include grammatical frame insertion, the ability to generate verbs, as well as the development of an internally generated sentence level plan. For the purpose of the current review we will focus on the latter three cognitive processes, as these skills can be examined at the single word level. Assessment of other cognitive processes such as grammatical frame insertion needs to be examined at the sentence level, and this will be assessed in future investigations.

\section{Sentence-level Planning: Verb Production}

First and foremost, in order to produce a sentence, a speaker must be able to successfully retrieve all the key lexical content elements, most particularly the main verb (verb retrieval), as it imposes powerful constraints on the structure of the sentence to be produced (for example, it determines whether a direct or indirect object can be included (see especially, Sloan Berndt et al.1997a, 1997b; Webster \& Whitworth, 20102). This ability can be disproportionately impaired after brain damage (for a review see Mätzig et al., 2009). Black and Chiat (2003) argue that nouns and verbs differ at both the conceptual-semantic and the syntactic level. At the conceptualsemantic level, verbs differ from nouns in their sensory richness and tightness of conceptual- 
semantic fit. Moreover, as several recent authors have pointed out, they contribute to the meaning of a sentence in a different way from nouns, because they specify not just the nature of an action, but also the entire event involving that action, including the participating roles of the entities described in the sentence (Marshall, Chiat, \& Pring, 1998). At the syntactic level, verbs vary as to the number of arguments they can take, and these impose significant constraints on the syntactic structure of the sentence. So for example, the verb "fell" requires an agent argument only (e.g. "the clown fell"), whereas "kick" requires a direct and an indirect object (e.g. the horse kicked the jockey"), while "send" requires three arguments: an agent, a theme and a goal, as in "Dean sent the car to the garage" (Agent is assigned to the subject Dean, Theme is assigned to the direct object the car, and Goal is assigned to the indirect object the garage) (Thompson, Shapiro, Li \& Schendel, 1995).

Deficits in verb retrieval have long been observed in a range of aphasic syndromes, and this most likely reflects the complexity of this linguistic skill. However, it has been reported on numerous occasions that patients with nonfluent aphasia (e.g., Broca's aphasia) have significantly greater impairments in verb than noun retrieval (for review see Mätzig, Druks, Masterson \& Vigliocco, 2009). In nonfluent aphasia, utterances are often reduced to one or two words, and are separated by long pauses, even though single word naming can be relatively well preserved (e.g., McCarthy \& Kartsounis, 2000; Schwartz \& Hodgson, 2002; Williams \& Canter, 1982). In a comprehensive review of the literature, Mätzig and colleagues (2009) explored the dissociation between verb retrieval as measured by a commonly used action-naming task and an object-naming task in 269 aphasia patients. Of the nonfluent aphasics in this sample $(\mathrm{N}=132)$, three-quarters showed poorer performance in verb naming than object naming. This was the highest proportion of dissociation when compared with other aphasic subtypes. In addition, 49 
aphasics had a dissociation of greater than $30 \%$ accuracy in object-naming compared to an action-naming task. Of these, $60 \%$ of patients were nonfluent aphasics (Mätzig, Druks, Masterson \& Vigliocco, 2009). Such observations, have led some to argue that deficits in verb retrieval can explain the difficulty in producing cohesive sentences in nonfluent aphasia (see Marshall, Pring \& Chiat, 1998). Specifically, it has been found that deficits in single word action naming tasks are associated with further impairments in sentence-level processing. For example, Thompson, Lange, Schneider, and Shapiro (1997) found that a group of 10 patients with nonfluent aphasia who were disproportionately poor at an action-naming task (when compared to object naming) were also disproportionately poor at producing sentences when they contained verbs with multiple argument structures; this impairment was much less evident in sentences that contained a verb with one argument structure.

\section{Sentence-level Planning: Goal-Driven Response Selection}

To produce longer utterances, a speaker must also manage their activation levels so that no item gets selected before it is supposed to. It has been suggested that there is a dedicated mechanism that performs this function, which operates by biasing the flow of activation, either enhancing, or inhibiting lexical representations based on the current production goal (see especially, January, Trueswell \& Thompson-Schill, 2009; Schnur, Schwartz, Brecher, \& Hodgson, 2006; Scott \& Wilshire, 2010; Speer \& Wilshire, 2013; Wilshire \& McCarthy, 2002). It has been suggested that such a mechanism may play a crucial role in managing competition between elements planned in the same utterance (see Martin et al., 1999).

As previously mentioned, patients with nonfluent aphasia have immense difficulty producing sentences, despite relatively well preserved single word production. It has been suggested that these patients have a deficit in this control system and therefore have difficulties 
managing competition between conflicting representations (Biegler, Crowther, \& Martin, 2008; Hamilton \& Martin, 2005; January et al., 2009; Schnur et al., 2006; Thompson-Schill, D’Esposito, Aguirre \& Farah, 1997; Wilshire \& McCarthy, 2002). Evidence in support of this theory comes from the performance of individuals with nonfluent aphasia on tasks that appear to make heavy competition-resolution demands. For example, several individuals with nonfluent aphasia have demonstrated prolonged naming latencies in the Stroop task, where a colour name is presented and the participant must identify its display colour while ignoring what the word actually says (Hamilton \& Martin, 2005; Scott \& Wilshire, 2010). This task would appear to involve inhibiting a potentially competing response - the word name - in order to produce the desired response. Such individuals may also exhibit a characteristic pattern of performance in the verb generation task, which involves generating an action that is associated with a given noun (e.g., scissors -> "cut"). Thompson-Schill and colleagues argue that when the noun offers several alternatives (e.g., rope -> "tie", "knot”, “pull”, “drag”), competition must be resolved between these alternatives before a single response can be selected (Thompson-Schill, D’Esposito, Aguirre, \& Farah, 1997; Thompson-Schill, D’Esposito, \& Kan, 1999). Using this task, CameronJones (2008) found that nonfluent aphasics exhibited a disproportionate difficulty with multiplealternative items, when compared to items where one single verb response is dominant (e.g., scissors $->$ “cut”).

Spontaneous connected speech also differs from simple single word tasks, such as object naming, in that it involves coming up with a message intention, which is then used to "drive" sentence production. This message intention if sufficiently strongly activated and maintained exerts top-down control of the language system, enabling goal-appropriate elements to be selected, and inappropriate elements to be rejected. A difficulty with this aspect of spontaneous 
production may also impact on a person's communicative abilities. One task that arguably involves spontaneously generating a very simple message intention is letter fluency, where the participant must generate as many words as they can think of which start with a particular letter. This task requires the participant to search through their mental lexicon in order to select appropriate lexical items that adhere to goals of the task (for review see Henry \& Crawford, 2004). There is a wealth of evidence to suggest that patients who suffer damage to the left frontal region of the brain show specific impairments in this task, (Baldo \& Shimamura, 1998; Rogers et al., 1998; Schwartz \& Baldo, 2001; Stuss et al., 1998) when compared to both patients with posterior lesions and controls (Pendleton et al., 1982; Perret, 1974). For example, Robinson, Shallice, Bozzali and Cipolotti (2012) found that letter fluency performance in 47 patients with a frontal lesion primarily due to stroke was significantly worse than 20 patients with a posterior lesion, and also when compared with 35 healthy controls. Additionally, they also found that patients with a left frontal lesion performed significantly more poorly in this task, compared to patients with a right frontal lesion. Additionally Papagno et al., (2012) also found that patients with a left frontal brain tumour were more likely to be impaired on this task than patients with a brain tumour in other anatomical regions.

The two hypothesised processes described here - the management of competition and "top-down" language control - may in fact turn out to be aspects of a single common capacity. For example, the ability to select the correct word for production when several candidates are currently activated may depend crucially on the strength of the "message intention". A strong propositional message intention may provide a conceptual framework that helps the speaker ensure that each word selected has the desired function. This in turn may help to minimise direct competition between the different elements planned for production in a single sentence. 
Therefore, for the purposes of this thesis, we will refrain from taking a position on this argument and instead refer to these various abilities collectively as "goal-driven response generation".

\section{Language Comprehension: Auditory Word Identification}

So far we have considered cognitive skills that are critical for language production - both at the level of the single word and the sentence level. We now turn to the question of language comprehension. Cognitive theories of auditory language comprehension suggest that this process can be broken down in similar ways to those depicted in theories of single word production.

Firstly, if we consider the simplest example - comprehending a single auditory word - cognitive theories make a clear distinction between the auditory word identification process, whereby the auditory stimulus is associated with a single known word, and the subsequent meaning retrieval process, whereby a semantic description of the stimulus word is generated (Hickok \& Poeppel, 2007; Marslen-Wilson \& Welsh, 1978; McClelland \& Elman, 1986; Norris, McQueen, \& Cutler, 2000). Virtually all these theories agree that the former involves identification of the acoustic properties of the speech signal, and the subsequent mapping onto the most appropriate word representation in the mental lexicon, which we will call auditory word identification. Many cognitive theories of auditory word identification argue that spoken words are processed as speech unfolds, and in doing so listeners attempt to map incremental segments of the acoustic signal onto a representation in the mental lexicon. For example, according to the cohort model, first proposed by Marslen-Wilson and Welsh (1978), the first few phonemes of a spoken word activate a set or cohort of word candidates that are consistent with that input. These candidates then compete with one another for activation. As more acoustic input is analysed, candidates that are no longer consistent with the input drop out of the set. This process continues until only one word candidate matches the input or the best fitting word may be chosen if no single candidate is 
a clear winner (e.g. Cole \& Jakimik, 1980; Dahan, Magnuson, Tanenhaus, \& Hogan, 2001; Marslen-Wilson, 1987; Marslen-Wilson \& Tyler, 1980; McClelland, Elman, \& Diego 1986; Norris, McQueen, \& Cutler, 2000; Taft \& Hambly, 1986; Tyler 1984; Vitevitch \& Luce, 1999). Neuropsychological studies have found that patients with Wernicke's Aphasia (WA), as well as patients with pure word deafness have profound deficits in auditory word identification (e.g. Caplan, Gow \& Makris, 1995). WA is an acquired language impairment characterised by severely impaired single word comprehension with fluent and discorded speech (Goodglass \& Kaplan, 1983; Goodglass, Kaplan, \& Barresi, 2001). A common task used in the literature to examine this deficit is single word repetition, as successful performance in this task relies on the ability to identify the acoustic properties of the to-be-repeated word. It has been extensively reported that patients with WA demonstrate significantly poor performance on this task. Patients with WA have normal articulation; therefore failure on this task cannot be attributed to an inability to articulate the word (for review see Robson, Grube, Lambon Ralph, Griffiths \& Sage, 2013). Another task commonly used is phoneme discrimination, in which participants have to determine if two spoken words are identical. The pairs are most commonly made to differ by one phonemic feature (e.g., "cap-tap"). It has been consistently demonstrated that patients with WA have marked difficulties with this task (e.g., Basso, Casati \& Vignolo, 1977; Baum, 2002; Blumstein, Baker, \& Goodglass ,1977; Miceli, Gainotti, Caltagirone \& Masullo, 1980; Tallal \& Newcombe, 1978). In one study, Robson and colleagues (2013) had 11 patients with WA complete the phoneme discrimination task. It was found that at a group level these patients were significantly worse on this task when compared to a group of 11 age and hearing-matched controls (Robson, Grube, Lambon Ralph, Griffiths, \& Sage, 2013). 


\section{Language Comprehension: Verbal Short-Term Memory}

Analogous to sentence production, additional skills may be required during the comprehension of connected speech. One of the most important of these is likely to be verbal short-term memory, since the lexical elements must be retained online until the relevant relational information is extracted (see Caplan \& Waters, 1999 for review). In their classic theory of working memory, Baddeley, Lewis, and Vallar (1984) proposed the existence of a phonological store, a buffer store that can hold phonological information for a few seconds. This idea of a specific form of phonological short-term memory plays an important role in language comprehension, and possibly also production. It is often measured by the digit span task but another task that might also provide a particularly pure measure of phonological short-term memory is nonword repetition (Gathercole \& Baddeley, 1989; Gathercole, Willis, Emslie \& Baddeley, 1992). According to this view, repetition of nonwords requires more reliance on the temporary storage of phonological representations in short-term memory because of the reduced availability of long-term lexical knowledge to support the unfamiliar phonological forms (Archibald \& Gathercole, 2007). In healthy subjects, performance in nonword repetition it is highly correlated with digit span performance (e.g., Gathercole \& Baddeley, 1989; Gathercole, Willis, Emslie, \& Baddeley, 1992; Gupta, 2003; Gupta, MacWhinney, Feldman \& Sacco, 2003). In regards to sentence comprehension, it has been suggested that phonological short-term memory may be of importance for understanding sentences where the thematic relations amongst sentence elements cannot be inferred from the overall context, but must be established on the basis of word order and other grammatical cues (e.g., Put the white key in on the red box) (Martin \& Romani, 1994; Martin \& Feher, 1990; Vallar \& Baddeley, 1984; Walters, Caplan \& Hildebrandt, 1991). It has been found that patients with deficits in phonological STM - as 
defined by poor performance on the digit span task - typically fail to comprehend these sentences, and this has been attributed to the absence of a phonological back up. For example, Gvion and Friedmann (2012) had patients with deficits in phonological STM comprehend a sentence with an ambiguous word, situated in a context that strongly biases its meaning. However, this word gets disambiguated toward a different meaning at a later point in the sentence (e.g., the toast that the elderly couple had every breakfast was always for happy life and for love). Gvion and Friedmann (2012) found that all patients with a deficit in phonological STM had severe deficits in comprehending these sentences.

\section{Reading}

So far, we have considered only spoken language processing. However, another language process that is fundamental to everyday language use is reading. Cognitive theories of reading are also in agreement that reading can be broken down into a number of more fundamental cognitive skills. Many of these cognitive skills overlap with those involved in oral language comprehension and production, but there are also some additional unique ones (for review see Rayner \& Reichle, 2010). There is considerable evidence within the cognitive literature to suggest that the process of identifying a written word based on its visual pattern requires a very different set of cognitive skills from those used in auditory word recognition (see Dehaene \& Cohen, 2011). We shall refer to these skills collectively as visual word form identification. In general, theories of visual word identification propose that when reading a word, skilled adult readers process the component letters of a word in parallel (Harm \& Seidenberg, 2004, 1999; Plaut, McClelland, Seidenberg \& Patterson, 1996; Seidenberg \& McClelland, 1989). Evidence for this view comes from the fact that readers tend to show very little effect of word length in single word reading tasks (for review see Barton, Hanif, Björnström \& Hills, 2014). In many 
such models, visual word recognition is viewed as a cascading process, in which the features of the individual letters, such as horizontal lines, diagonal lines, and curves, activate their corresponding letter representations in parallel, which in turn activate the representations of words that contain those letters in similar positions. The most highly activated word is likely to be that which possesses all the required letters in the appropriate position (e.g., Harm \& Seidenberg, 2004, 1999).

A specific deficit in this cognitive skill is a hallmark feature of pure alexia, an inability to recognise visually presented words, despite preservation of other visual and cognitive abilities (e.g. Farah \& Wallace, 1991). Pure alexia is characterised by a very slow reading rate, with patients often appearing to identify each consecutive letter individually in order to "spell the word out" ("letter-by-letter reading"; see Hanley \& Kay, 1996). Further evidence for the use of a letter-by-letter strategy is that reading time increases incrementally with the number of letters in a word (see especially Behrmann, Black \& Bub, 1990). Although a number of theories have been postulated in the literature to account for this phenomenon, one such account proposes that in pure alexia there is a loss in the ability to recognise letters simultaneously (as described above; Behrmann, Shomstein, Black \& Barton 2001; Farah \& Wallace, 1991).

Once the appropriate visual representation for the word has been identified, dual process theories propose two main sets of subsequent processes are needed to actually pronounce the word or extract its meaning. One set capitalises on the reader's knowledge of the relationships between orthography and phonology, and applying this knowledge to generate a pronunciation of the word. This knowledge may consist of a set of correspondence rules reflecting the most common pronunciations of each letter or letter unit in a given context (e.g., Coltheart, Curtis, Atkins, \& Haller, 1993; Coltheart et al., 2001; Perry, Ziegler \& Zorzi, 2007; Zorzi, Houghton \& 
Butterworth, 1998), or of knowledge generalised from the pronunciations of similarly spelled words (e.g., Plaut et al., 1996; Seidenberg \& McClelland, 1989). These processes are therefore likely to be sensitive to the sublexical structure of the word, such as the regularity or consistency of its spelling and its length in letters. We refer to this set of processes here as orthographicphonological mapping.

The other set of processes capitalises on the reader's knowledge of the stimulus word's specific identity and/or meaning. For example, in Coltheart and colleagues (2001) model, familiar combinations of letters activate an orthographic representation of the word, which in turn activates information about its phonological form and meaning (see also Coltheart et al., 1993; Perry et al., 2007; Zorzi, Houghton \& Butterworth,1998). In Harm and Seidenberg's (2004) model, the orthographic representation of the word is mapped directly onto its semantic representation (see also Plaut et al., 1996). These processes are therefore likely to be more sensitive to word-level properties, such as a word's frequency of occurrence and its meaning. We refer to this set of processes as orthographic-sematic mapping.

These models eloquently explain the double dissociation that occurs in two reading disorders: phonological and surface dyslexia (Coltheart, 1985). Phonological dyslexia is a condition in which following brain damage to a previously skilled reader, there is a selective deficit in the ability to read nonwords (see Funnell, 1983). Nonwords have no representation within the lexicon; consequently to correctly read the words aloud, orthographic-phonological manning is required. This disorder is consistent with a selective deficit involving this set of processes (Coltheart, et al., 2001; Coltheart, Curtis, Atkins \& Haller, 1993; Coltheart \& Rastle, 1994). In surface dyslexia, in which following brain damage to a previously skilled reader, there is a specific deficit in the ability to read irregular words (e.g. yacht (Behrmann \& Bub, 1992; 
McCarthy \& Warrington, 1984)). Irregular words will be read incorrectly if one conforms to the mapping of its grapheme to its phoneme. Consequently, to read these words aloud, the visual input must be mapped to its meaning. This disorder has been interpreted as a selective impairment to the orthographic-semantic mapping processes (Coltheart, et al., 2001; Jobard, Crivello, \& Tzourio-Mazoyer, 2003).

In conclusion, our brief theoretical review of the cognitive literature has identified 11 distinct language skills that are likely to be critical for various types of language communication - including production, comprehension and reading. Furthermore, a deficit in each language skill results in a unique neurological profile, providing further confirmation that each skill is a separate entity. The list of cognitive skills is by no means exhaustive, but it does identify those skills that are agreed upon most in the literature, and for which the most supportive evidence exists. 
Table 1.2

A summary of the core cognitive skills identified from cognitive theories of language and a description of the language profile in patients with a

deficit to each skill

Cognitive Skill

Description

Specific Impairments

Language Profile

In:

\begin{tabular}{|c|c|c|}
\hline $\begin{array}{c}\text { Accessing Semantic } \\
\text { Knowledge }\end{array}$ & $\begin{array}{l}\text { Retrieving the meaning e.g. obtaining } \\
\text { information about the semantic } \\
\text { category, function, colour, size, etc. } \\
\text { of the target item }\end{array}$ & Semantic Dementia \\
\hline
\end{tabular}

Lexical Selection

Selecting the appropriate word from the mental lexicon that best matches the semantic concept in mind

Phonological Encoding

Retrieving information about the selected word's sound form from the mental lexicon Articulatory Motor
Planning

Verb Retrieval
Classical Anomia

Conduction Aphasia

Apraxia of Speech

Nonfluent Aphasia, particularly Broca's aphasia
Constructing a motor plan for the articulatory execution of that item

The argument structure of a verb is a crucial component of sentence production, and deficits in the ability to retrieve a verb, has a cascading impairment on this process
Production of semantic errors in picture naming, low category fluency score relative to letter fluency, confusions between semantically related items in tasks that involve matching a spoken word to a picture

Strong frequency effect in picture naming and disproportionately high production of omission errors. Good performance on comprehension tasks such as the picture word matching task

Strong length effect in in picture naming and disproportionately high production of phonological errors. Poor performance in single word repetition

Impaired rate of speech - both spontaneous and in more constrained word and phrase recitation tasks and articulatory errors

Deficits in action naming, and specific impairments when producing a sentence with multiple argument structures 
Cognitive Skill

Goal Driven Response Selection

Auditory Word

Identification

Phonological Short Term Memory

Visual Word Form Identification

Orthographic-

Phonological Mapping

Orthographic-Semantic Mapping
Description

Using a sentence plan to drive the selection of the correct word to incorporate into a sentence, when several candidates are activated

Identification of the acoustic properties of a speech signal, and the mapping onto the most appropriate word representation in the mental lexicon

The ability to maintain the phonological representations of words in a temporarily active state over short intervals

Identifying a written word based on its visual pattern

The relationships between orthography and phonology, and applying this knowledge to generate a pronunciation of the word

Knowledge of the to-be-read word's specific identity and/or meaning

Specific Impairments

In:

Language Profile

Nonfluent (e.g., Broca's

Aphasia) and more generally in patients with left frontal damage

Wernicke's Aphasia

(specifically, pure word deafness)

Phonological STM deficits/conduction aphasia

Pure Alexia

Phonological Dyslexia

Surface Dyslexia
Deficits in tasks that require the resolution of conflict e.g. verb generation and Stroop; and deficits in tasks that require strategic search e.g. letter fluency

Deficits in single word repetition and auditory comprehension tasks e.g. picture word matching; and difficulties in discriminating between phonological auditory stimuli

Reduced digit span. Highly disproportionate deficit in nonword repetition relative to real word repetition.

Selective disorder of word reading (spoken language may be unaffected). Abnormal word length effect, characterised by longer word reading times for longer words.

Disproportionately poor nonword reading

Disproportionately poor reading of irregular words compared to regular words 


\section{Neuroanatomy of the Core Cognitive Skills}

One of the key advantages of adopting a core skills approach is that it is likely to offer greater power to discriminate amongst different language profiles based on the localisation of the person's tumour. Therefore, it is important to be able to demonstrate that each skill is reliably associated with a distinct set of neural structures. In order to identify these neural structures, we reviewed the literature to identify the cortical structures essential to the cognitive skill in question. This was done in two ways. First, we selected a number of tasks/manipulations that are arguably highly sensitive at assessing each cognitive skill. Using this information we can then select studies that have used neuropsychological methodology to determine what specific brain regions are most reliably associated with a deficit in each hypothesised skill. Where possible, we selected neuropsychological studies that had clearly operationalised a particular cognitive skill, in a manner consistent with conceptualisation of the process in question, as summarised above. In order to have consistent evidence across each cognitive skill, emphasis was placed on largegroup lesion mapping studies, and those that used statistical methods to infer lesion-behaviour relationships, such as voxel-based lesion symptom mapping (VLSM; Bates et al., 2003) and voxel-based morphometry (VBM; Ashburner \& Friston, 2000). Second, if no such studies existed, we relied on other methodological techniques, such as more descriptive lesion overlap analyses and fMRI studies. Table 1.3 presents a comprehensive summary of how conclusions were derived regarding the critical neural regions associated with each task/manipulation involved in each core skill, and also briefly describes the most important sources of evidence that supported these conclusions. 
Table 1.3

A summary of the cortical regions associated with each cognitive skill assessed by the BLAST ${ }^{2}$

\begin{tabular}{ll}
\hline Cognitive Skill & \multicolumn{1}{c}{ Cortical Structures } \\
\hline $\begin{array}{l}\text { Accessing } \\
\text { Semantic }\end{array}$ & Left temporal pole \\
Knowledge & \\
& \\
& Left anterior temporal lobe \\
& (specifically left anterior middle \\
& temporal gyrus), superior temporal \\
& sulcus, and in white matter tracts deep \\
& to the sulcus
\end{tabular}

Left temporal lobe: BA 22 (superior temporal gyrus), 37 (fusiform gyrus), 38 (temporopolar area), 41, and 42 (auditory cortex)

\section{Key Evidence}

Picture word matching and category fluency scores both significantly correlated with the amount of cortical atrophy to this region in patients with semantic dementia (SD). Cortical atrophy was determined in six patients with SD using VBM (Mummery, Patterson, Price, Ashburner, Frackowiak \& Hodges, 2000)

Regions predictive of the production of semantic errors in picture naming in a large group VLSM study of patients with left hemisphere lesions mainly due to stroke (Schwartz et al., 2009), and in a follow-up study of this population, in which the classification of semantic errors was expanded to include mixed errors, related non-nouns and semantic circumlocutions (Walker et al., 2011).

Regions predicting poor category fluency in a left hemisphere stroke population using VLSM. Performance in letter fluency was subtracted from category fluency scores. (Baldo, Schwartz, Wilkins \& Dronkers, 2006)

\section{Conclusion for Accessing Semantic Knowledge: Left Anterior Temporal Region}

\section{Lexical Mid to posterior portion of the left \\ Selection middle temporal gyrus (MTG),} with some extension into the white matter just medial to left MTG

Left BA37: posterior inferior temporal cortex, and posterior angular gyrus; borders with middle temporal gyrus,
Regions critically predicting poor performance on the Boston Naming Test, in a left hemisphere stroke population after partialling out any effects of articulatory fluency (using examiner's rating of spontaneous speech), and visual recognition (using recognition scores on the BNT) (large group VLSM study: Baldo, Arévalo, Patterson, \& Dronkers, 2013)

Tissue dysfunction in a large sample of acute stroke patients most strongly correlated with a measure of modality-independent lexical access. Operationalised by greater than $10 \%$ errors in oral naming of pictures, tactile naming and written naming, and less than $10 \%$ errors in pictureword verification (see Deleon et al., 2007)

\begin{tabular}{ll}
\hline Conclusion for Lexical Selection: Left Posterior Tempo \\
\hline $\begin{array}{l}\text { Phonological } \\
\text { Encoding }\end{array}$ & $\begin{array}{l}\text { Left: postcentral gyrus, inferior } \\
\text { portion of the precentral gyrus, and } \\
\text { supramarginal gyrus, and the white }\end{array}$
\end{tabular}

Production of phonemic paraphasias (phonological errors) on the Philadelphia naming test (large group VLSM study of patients with left hemisphere lesions mainly due to stroke; Schwartz, Faseyitan, Kim, \& Coslett, 2012)

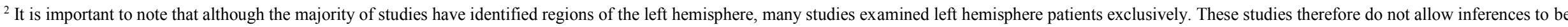
made about the degree of right hemisphere involvement
} 
matter tracts of the arcuate

fasciculus

Left superior temporal cortex (including Heschl's gyrus) and inferior parietal (angular and supramarginal gyrus)

Left: supramarginal gyrus, postcentral gyrus, precentral gyrus and insula

Left BA39: angular gyrus, superior temporal sulcus and inferior parietal lobe

Left superior temporal gyrus, close to the border with inferior parietal cortex
Regions predicting performance on word repetition and nonword repetition task. VLSM analysis using 84 patients with a left hemisphere stroke (Baldo, Katseff \& Dronkers, 2012)

Regions predicting the low scores of the p-parameter, a measure of the effectiveness of mapping between lexical and phonological representations, as outlined in the Dual Route Two Step Model (see Dell et al., 1997), a VLSM study. Predictions made using computational modeling (Dell, Schwartz, Nozari, Faseyitan \& Branch Coslett, 2013). Participants consisted of a large sample of left hemisphere patients used in a previous investigation (Schwartz et al., 2012),

Tissue dysfunction in a large sample of acute stroke patients most strongly correlated with a measure of phonological word form encoding. Operationalised using the following criteria: greater than $10 \%$ errors in tactile naming and oral naming, and less than $10 \%$ errors in written naming of pictures and semantic errors, and phonological errors, and or circumlocutions in at least one of the above tasks (see Deleon et al., 2007)

Regions predicting performance on word repetition task. VLSM analysis using 84 patients with a left hemisphere stroke (Baldo, Katseff \& Dronkers, 2012)

\section{Conclusion for Phonological Encoding: Left Posterior Temporal-parietal Regions}

Auditory Word Left posterior superior temporal

Identification sulcus/gyrus and inferior supramarginal gyrus, and inferior pre and postcentral gyrus

Left posterior temporal-parietal regions, especially the posterior superior temporal gyrus

Superior temporal gyrus, planum temporale, junction of the parietal
Regions associated with errors on an auditory discrimination task (ADT) (Martin, Schwartz, \& Kohen, 2006) (large group VLSM study of patients with left hemisphere lesions mainly due to stroke: Schwartz et al., 2012). ADT involved subjects hearing two recorded words in succession and indicating whether or not the words were identical

Regions uniquely associated with poor performance in phonological discrimination using the PALPA word and nonword minimal pair discrimination task (PALPA 1 \& 2:6, Lesser, \& Coltheart, 1992) in 10 patients with Wernicke's Aphasia (Robson, Sage \& Lambon Ralph, 2012)

Regions predicting the operationalisation of mapping between auditory input and phonological representations ( $n l$ parameter) using VLSM as outlined in the Dual Route Two Step Model, (see 


\section{Cognitive Skill Cortical Structures}

and temporal lobes (area SpT), as

well as supramarginal gyrus and

postcentral gyrus

\section{Key Evidence}

Dell et al., 1997). Predictions made using computational modeling (Dell, Schwartz, Nozari, Faseyitan \& Branch Coslett, 2013). Participants consisted of a large sample of left hemisphere patients used in a previous investigation (Schwartz et al., 2012),
Conclusion for Auditory Word Identification: Left Poste
Verbal STM Left posterior MTG and STG and inferior parietal cortex (angular and supramarginal gyrus)

Large portion of left STG, from the superior temporal pole to posterior STG as well as Heschl gyrus. Also extensions into left MTG and angular and supramarginal gyrus

Conclusion for Verbal STM: Left Posterior Temporal-parietal Regions

\begin{tabular}{lll} 
Conclusion for Verbal STM: Left Posterior Temporal-parietal Regions \\
\hline $\begin{array}{l}\text { Goal-driven } \\
\text { response }\end{array}$ & BA 4 (Primary Motor), 6 & Regions predicting poor letter fluency in a left hemisphere stroke population using voxel lesion \\
selection & (Premotor), 44 (pars opercularis), & symptom mapping. Performance in category fluency was subtracted from letter fluency scores
\end{tabular}

Somatosensory Cortex), 39

(Angular Gyrus), 40)

Left inferior and middle frontal gyrus and the anterior cingulate gyrus

Left ventrolateral prefrontal cortex, specifically the left inferior frontal gyrus, and underlying white matter

Left inferior frontal gyrus

Left inferior frontal gyrus
Regions predicting performance on Digit and Word Span. VLSM analysis using 84 patients with a left hemisphere stroke (Baldo, Katseff \& Dronkers, 2012)
Regions predicting performance on a non word repetition task. VLSM analysis using 84 patients with a left hemisphere stroke (Baldo, Katseff \& Dronkers, 2012)

Meta-analysis of 28 studies with 490 subjects on brain activation using fMRI using letter fluency (Wagner, Sebastian, Lieb, Tüscher, \& Tadić, 2014)

Regions predicting the size of the Stroop effect in reaction times (RT) (incongruent RTcongruent RT) in the Stroop task VLSM analyses of 45 patients with a frontal lobe lesion (Tsuchida \& Fellows, 2012).

Patients with damage to this region had significantly slowed reaction times in the high selection demands in a verb generation task (Thompson-Schill, D’Esposito, Aguirre, \& Farah, 1997)

Region predicting inhibitory effect of near semantic neighbours in a picture naming task (compared picture naming accuracy between items with many and few semantic neighbours). VLSM subtraction analysis of large sample of left hemisphere stroke (Mirman \& Graziano, 2013) 
Cognitive Skill

Articulatory

Motor

Programming

\section{Cortical Structures}

Left: Anterior insula, inferior

frontal gyrus, as well as the adjacent white matter and basal ganglia, and the anterior temporal areas

Left anterior insular, left frontal posterior regions anf left caudate

Left anterior insula

\section{Key Evidence}

Brain regions predicting performance in articulation and prosody subtests of the Aachen Aphasia Test in 102 left hemisphere stroke patients using VLSM (Henseler, Regenbrecht \& Obrig, 2014)

Slow rate of speech in patients with apraxia of speech was associated with atrophy in this cortical regions (Ogar, Dronkers, Brambati, Miller, \& Gorno-Tempini, 2007)

Brain region predicting articulation deficits in 25 stroke patients using computerised lesion overlap. Articulatory behaviour was assessed by analysing articulatory inconsistencies on repeated utterances, rhythm, stress and intonation, effortful trial-and-error, and articulatory movements by two specially trained speech-language pathologists (Dronkers, 1996)

\section{Conclusion for Articulatory Motor Planning: Left Insula and Left Inferior Frontal Regions}

Verb Retrieval Left Inferior frontal area (BA 45, 47)

Left: BA 44 (pars opercularis), BA 45 (pars triangularis)

\section{Conclusion for Verb Retrieval: Left Inferior Frontal Regions}

Visual Word Left fusiform gyrus

Recognition

Left fusiform gyrus

Left fusiform gyrus and inferior occipital gyrus
Regions associated with errors in an action-naming task when compared to an object naming task, using VLSM in 16 left hemisphere stroke patients (Piras \& Marangolo, 2007)

Regions predicting performance in a verb naming task when compared to an object naming task, using VLSM in a sample of 20 left hemisphere stroke patients (Piras \& Marangolo, 2010) reading tasks (Starrfelt, Habekost \& Leff, 2009)

Brain region associated with abnormal length effect slope using reaction times for each word length normalised according to overall RT. VLSM analysis of 20 patients with a left posterior lesion (Roberts et al., 2012)

Region of overlap in three cases of pure alexia (criteria: length effects in reading, occasionally preserved letter naming and backward-spelling reading procedure, and mild or no oral language impairment) (Ripamonti et al., 2014)

Conclusion for Visual Word Identification: Left Fusiform Gyrus

Orthographic-

Phonological Mapping

\section{Middle and inferior frontal gyrus,} angular gyrus, middle occipital and temporal gyrus, supramarginal gyrus and pre and postcentral
Regions predicting nonword reading performance using VLSM in 331 acute stroke patients, with testing within 24hours of assault (Cloutman et al., 2011) 
gyrus; as wells as the superior

longitudinal and superior frontooccipital fasciculi

Left posterior superior temporal gyrus, supramarginal gyrus, inferior frontal gyrus, precentral gyrus and insula

Posterior superior and middle temporal gyri, fusiform gyrus and the inferior parietal lobule

Insula, left inferior frontal gyrus, rolandic operculum and precentral gyrus
Impaired nonword reading was significantly associated with lesions to these brain regions. VLSM analysis of 70 patients with left hemisphere stroke (DeMarco, Rising, Wilson, Rapcsak, \& Beeson, 2012)

Nonword word reading accuracy is positively correlated with gray matter volume in these brain regions in 56 patients with neurodegenerative disease (Ogar et al., 2009)

Regions of overlap in a sample of 33 individuals with phonological dyslexia, defined as significantly poorer nonword than real word reading (Ripamonti et al., 2014))

\section{Conclusion for Orthographic-Phonological Mapping: Left Posterior Temporal-parietal Regions and Left Inferior Frontal Regions}

\begin{tabular}{ll}
\hline $\begin{array}{l}\text { Orthographic- } \\
\text { Semantic } \\
\text { Mapping }\end{array}$ & $\begin{array}{l}\text { Left anterior temporal pole, anterior } \\
\text { superior and middle temporal gyrus } \\
\text { and the fusiform gyrus }\end{array}$ \\
& $\begin{array}{l}\text { Left superior temporal gyrus, } \\
\text { middle temporal gyrus, left inferior } \\
\text { temporal gyrus, left middle } \\
\text { occipital gyrus, left insula and left } \\
\text { inferior occpito-frontal insula. }\end{array}$ \\
\hline
\end{tabular}

Irregular word reading accuracy is positively correlated with gray matter volume in these brain regions in 56 patients with neurodegenerative disease (Ogar et al., 2009)

Regions of overlap in five patients with surface dyslexia. Participants in this sample spoke Italian, a language that has an absence of irregular words. Surface dyslexia was therefore assessed by the rate of stress errors in three (or more) syllable words (Ripamonti et al., 2014) 


\section{Chapter 2: Introduction to the Current Study}

This thesis aims to explore the linguistic capabilities of a large cohort of surgical brain tumour patients using a newly designed assessment protocol: the Brief Language Assessment for Surgical Tumours (BLAST). The BLAST is a theory-driven assessment battery. It aims to assess key language skills identified by cognitive theories of language. These skills are viewed as fundamental cognitive building blocks for everyday language behaviours. The skills assessed have been selected because there is widespread agreement throughout the literature for their existence, and converging evidence regarding their specific neural localisation. Specifically, the BLAST aims to assess the following cognitive skills crucial for language production (both at a single word and sentence level) accessing semantic knowledge, lexical selection, phonological encoding, articulatory-motor planning, verb retrieval, goal-driven response selection and verbal short-term memory. The BLAST also aims to assess cognitive skills that make a unique contribution to language comprehension (both at a single word and sentence level): auditory word identification and verbal short-term memory; and to single word reading (at the single word level): visual word identification, orthographical-phonological mapping, and orthographicalsemantic mapping. The 11 cognitive skills assessed by the BLAST are presented in Figure 2.1. The primary benefit of this approach is that it may prove to be considerably more sensitive at detecting mild linguistic deficits, and also likely to be more effective at discriminating between different types of functional difficulties associated with different skills and neural structures. 


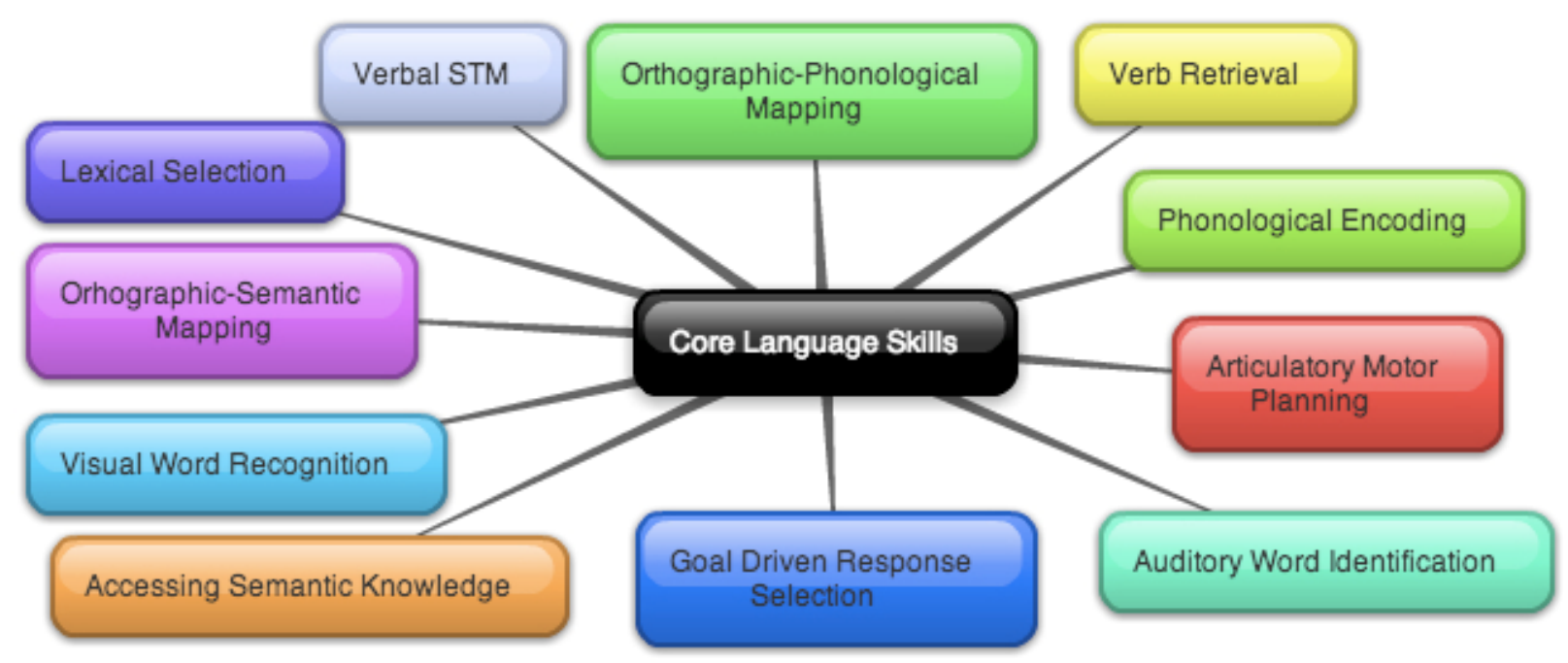

Figure 2.1. The set of 11 core language skills derived from current cognitive theories of language that are assessed in the BLAST.

By adopting a core skills approach, the BLAST has the potential to discriminate language profiles based on the anatomical localisation of the brain tumour. This is due to the finding that each core skill has specific neural underpinnings (see Table 1.3 for a comprehensive review). At the broadest level, the cognitive skills assessed by the BLAST can be categorised into the following two groups: the anterior group consisting of articulatory motor planning, verb retrieval, and goal driven response selection; and the posterior group consisting of: lexical selection, accessing semantic knowledge, auditory word identification, verbal short termmemory, visual word identification and orthographical-semantic mapping (orthographicalphonological mapping is the only cognitive skill that has not been categorised into a group, due to the extensive network of both anterior and posterior cortical regions involved in this skill. Classification into one of these groups would therefore be inappropriate). ${ }^{3}$ The next section will

\footnotetext{
3 We are aware that some neuropsychological studies found significant cortical structures in both frontal and posterior regions. Categorisation was based on cortical structures that were associated with the cognitive skill in all the studies reviewed and/or those that are most strongly associated with deficits involving that skill. It is important to note though, that this is just a broad categorisation for anatomical simplicity in order to investigate the BLAST's ability to discriminate language profiles; it is by no means definitive and mutually exclusive.
} 
outline the tasks selected to assess the 11 cognitive skills identified above. It will also provide a detailed description of the methodology used to operationalise each of these core skills from the tasks selected for the battery.

\section{The Brief Language Assessment for Surgical Tumours (BLAST)}

One of the most important considerations we had in mind whilst selecting tasks for the BLAST was to keep the entire battery as quick as possible to administer, but maintain its sensitivity. One way of minimising testing time is to assess more than one skill per test, and to tease these skills apart by manipulating different types of stimulus properties. These manipulations can then be used to derive multiple measures that can then be used to quantify each cognitive skill. In order to achieve this we have standardised each measure, allowing for multiple measures from different tasks to be combined into a single entity. For example, one of our tests selected was a single picture naming task, which was designed in such a way that effects of frequency and word length on naming accuracy could be separately assessed (frequency is a variable that been associated with the lexical retrieval stage of word production (e.g. Nickels \& Howard, 2001), whereas word length has been associated with phonological encoding (e.g., Levelt, 1999)).

In order to establish which tests to include in the assessment, and what kinds of manipulations to incorporate within them, we modelled language profiles of patients who have selective deficits in each of the core cognitive skills (see Table 1.2 for a comprehensive review). Wherever possible, we aimed to operationalise the relevant skill by using a conjunction of observations across multiple tasks, in order to maximise the selectivity of each measure. Our third and final consideration was wherever possible, we designed alternative versions of the tests, so as to minimise practice effects between the pre- and postoperative assessment phases. The 
eight tasks selected for the BLAST that adhere to these considerations are presented in Table 2.1.

The next section will describe in detail how these eight tasks are used to operationalise the 11 core cognitive skills assessed by the BLAST.

Table 2.1

The eight tests selected for inclusion in BLAST, and where relevant, the variables that were manipulated within each.

\begin{tabular}{|c|c|c|}
\hline Test & Description & Stimulus Manipulation \\
\hline $\begin{array}{l}\text { 1. Picture Naming } \\
\text { (Wilshire, 2002) }\end{array}$ & $\begin{array}{l}\text { Produce the name of a visually } \\
\text { displayed object, animal or } \\
\text { person. }\end{array}$ & $\begin{array}{c}\text { Length (monosyllabic, bisyllabic and } \\
\text { polysyllabic), Frequency (high, } \\
\text { medium, low) }\end{array}$ \\
\hline $\begin{array}{l}\text { 2. Verb } \\
\text { Generation } \\
\text { (Thompson-Schill } \\
\text { et al., 1997) }\end{array}$ & $\begin{array}{l}\text { View a pictured item, and } \\
\text { produce the name of an action } \\
\text { associated with that item }\end{array}$ & $\begin{array}{l}\text { Noun-verb association strength; where } \\
\text { half the items have a strong verb } \\
\text { associated with the noun (strong), and } \\
\text { the other half have many verbs } \\
\text { associated with the noun (weak) }\end{array}$ \\
\hline $\begin{array}{l}\text { 3. Picture Word } \\
\text { Verification } \\
\text { (Breese \& Hillis, } \\
\text { 2004) }\end{array}$ & $\begin{array}{l}\text { View a pictured item, and judge if } \\
\text { an auditory presented word is the } \\
\text { correct name for that item }\end{array}$ & $\begin{array}{c}\text { Semantically related judgements (e.g. } \\
\text { see horse but hear "deer") and } \\
\text { phonological judgements (e.g. see } \\
\text { cattle hear "cannon" }\end{array}$ \\
\hline $\begin{array}{l}\text { 4. Repetition } \\
\text { (Kay, Lesser, \& } \\
\text { Coltheart, 1996) }\end{array}$ & $\begin{array}{l}\text { Immediately repeat individual } \\
\text { auditory presented words }\end{array}$ & $\begin{array}{c}\text { Lexical status (real words and } \\
\text { nonsense words) }\end{array}$ \\
\hline $\begin{array}{l}\text { 5. Reading (Kay, } \\
\text { Lesser, \& } \\
\text { Coltheart, 1996) }\end{array}$ & $\begin{array}{l}\text { Read aloud individual written } \\
\text { words }\end{array}$ & $\begin{array}{l}\text { Length in letters, spelling-sound } \\
\text { regularity, lexical status (words vs. } \\
\text { nonsense words) }\end{array}$ \\
\hline $\begin{array}{l}\text { 6. Stroop (Stroop, } \\
\text { 1935) }\end{array}$ & $\begin{array}{l}\text { Ignore the identity of a word, and } \\
\text { name the colour it is presented in }\end{array}$ & $\begin{array}{l}\text { Congruency; the colour of the word } \\
\text { matches it's identity (congruent) or } \\
\text { does not match }\end{array}$ \\
\hline $\begin{array}{l}\text { 7. Fluency (Lezak, } \\
\text { 1982) }\end{array}$ & $\begin{array}{l}\text { Produce as many lexical items as } \\
\text { possible within one minute }\end{array}$ & $\begin{array}{c}\text { Letter (F, A, S), Category (animals, } \\
\text { fruit) }\end{array}$ \\
\hline $\begin{array}{l}\text { 8. Articulatory } \\
\text { Agility (Kaplan, } \\
\text { Goodglass \& } \\
\text { Barresi, 2001) }\end{array}$ & $\begin{array}{l}\text { Repeat a simple verbal sequence } \\
\text { as many times as possible within } \\
\text { five seconds }\end{array}$ & - \\
\hline
\end{tabular}




\section{Measurement of the Core Cognitive Skills}

The following section will outline how each of the core cognitive skills are operationalised using the BLAST. It will also summarise the key evidence used in determining the operationalisation methodology.

\section{Accessing Semantic Knowledge}

The patient group used to operationalise this cognitive skill was semantic dementia, as this population exhibits a pattern of performance that is strongly suggestive of a difficulty in retrieving semantic knowledge across multiple modalities. This includes word production and comprehension, and even in nonverbal picture-picture matching tasks (e.g. Pijnenburg, Gillissen, Jonker, Scheltens, 2004; Thompson, Patterson, \& Hodges, 2003). It has been repeatedly observed that patients with this profile exhibit the following three features: 1) semantic errors in picture naming (Garrard, Perry \& Hodges, 1997; Hodges, Graham \& Patterson, 1995; Jefferies \& Lambon Ralph, 2006); 2) poor category fluency relative to letter fluency (e.g., more difficulty listing animal names than words starting with a specified letter (Bozeat, Lambon Ralph, Patterson, Garrard \& Hodges, 2000; Garrard, Perry \& Hodges, 1997; Rascovsky, Salmon, Hansen, Thai, \& Galasko, 2007;); and 3) disproportionately poor performance in picture-word matching tasks when the distractor items are semantically related to the target (for example, pointing to a picture that shows "cat" from amongst an array of pictures of domestic animals; see esp. Corbett, Jefferies, Ehsan \& Lambon Ralph, 2009). These three criteria formed the basis of our assessment of accessing semantic knowledge. However, instead of using a conventional picture-word matching task, we used a picture-word verification task. This was motivated by the findings of Breese and Hillis (2004) who reported that a picture-word verification task, which assesses each target picture in the context of a range of different types of auditory distractors (semantically related, phonologically related, and unrelated), was more sensitive at detecting deficits in left 
hemisphere stroke patients than the picture-word matching task. In our assessment, the specific measure we used to assess semantic retrieval was the difference in scores on semantically related distractor-target pairs relative to phonologically related pairs. This difference score effectively eliminates the phonological (sound processing) component of this task, further ensuring that we are selectively assessing the semantic demands of the task.

\section{Lexical Selection for Production}

The aphasia profile most consistent with a selective deficit in lexical selection for production is that of patients with pure or "classical" anomia (Andreetta, Cantagallo \& Marini, 2012; Butterworth, 1992; Lambon Ralph, Sage \& Roberts, 2000; McNeil, Odell, \& Tseng, 1991;). It has been repeatedly observed that patients with this profile exhibit poorer performance in naming low frequency items when compared to higher frequency items in a picture naming task (e.g., case AW: Jacobs, Singer \& Miozzo, 2004; case FR: Avila, Lambon Ralph, Parcet, Geffner \& Gonzalez-Darder, 2001). Also these patients tend to produce a disproportionately high number of both omissions and semantic errors during this task (Lambon Ralph, Sage \& Roberts, 2000; Miceli, Amitrano, Capasso \& Caramazza, 1996). Therefore, for the operationalisation of lexical selection, we combined the following measures: 1) the slope of the frequency effect in picture naming, 2) the incidence of omission errors in picture naming, and 3) incidence of semantic errors in picture naming. It must be noted that since patients with pure anomia have relatively good language comprehension (Franklin, Howard \& Patterson, 1995; Gonon, Bruckert \& Michel, 1989; Howard, 1995; Laine, Kujala, Niemi, \& Uusipaikka, 1992; Raymer et al., 1997), we only looked at poor performance in the task/manipulations mentioned above relative to performance in a language comprehension task: picture word verification. Specifically, scores for lexical selection were only considered if the combined scores for the measures outlined above were below the standardised score for picture-word verification. 


\section{Phonological Encoding}

The patient group used to operationalise this skill was patients with conduction aphasia, as this population exhibits a pattern of performance that is strongly suggestive of a deficit in the ability to retrieve information about the sound form of a desired lexical item (see Buchsbaum, Baldo, Okada, Berman, Dronkers, Esposito \& Hickok, 2011). There is a wealth of evidence that these patients exhibit the following features: 1) length effects in picture naming (Caplan, Vanier, \& Baker, 1986; Kohn \& Smith, 1993, 1995; Pate et al., 1987; Wilshire \& McCarthy, 1996; Wilshire, 2002), 2) phonological errors in picture naming (Kohn \& Goodglass, 1985; see also Butterworth, 1992; Caplan, Vanier \& Baker; Kohn \& Smith, 1986; Pate, Saffran \& Martin, 1987; Pradat-Diehl et al., 2001; Wilshire \& McCarthy, 1996); and 3) poor performance in word and nonword repetition task (Strub \& Gardner, 1974; Caplan, Vanier \& Baker, 1986; Caramazza, Basil, Koller \& Berndt, 1981; Dell, Schwartz, Martin \& Saffran 1997). These criteria formed the basis of our assessment of phonological encoding. The inclusion of both words and nonwords in our repetition task enhances the likelihood we are assessing the phonological demands of the task. If only real words were included it is possible that poor performance in this task could be a result of deficits in mapping of the lexical item to its associated meaning, rather than a pure phonological encoding deficit. The inclusion of both stimulus ensures we are assessing the phonological demands of the task. In addition, for this measure we used time taken to repeat the item (latency) rather than accuracy, as it has been argued that repetition and nonword repetition latency is a more sensitive measure of ease of phonological encoding (see Vitevitch \& Luce, 1998; 2005). For the operationalisation of phonological encoding it is also vital that we account for any articulatory deficits that could confound performance in the task/manipulations described above. Consequently the average score from the above 
task/manipulations were subtracted from a measure of articulatory agility, in order to control for this possible confound.

\section{Auditory Word Recognition}

The aphasia profile most consistent with a selective deficit in auditory word recognition is that of patients with Wernicke's or fluent aphasia (e.g. Caplan, Gow \& Makris, 1995). Patients with WA consistently exhibit difficulties in identifying speech sounds, which translates to poor performance in single repetition tasks (for review see Robson, Grube, Lambon Ralph, Griffiths \& Sage, 2013), but also deficits in the ability to discriminate similar speech sounds (Basso, Casati \& Vignolo, 1977; Baum, 2002; Blumstein, Baker, \& Goodglass, 1977; Miceli, Gainotti, Caltagirone \& Masullo, 1980; Tallal \& Newcombe, 1978). These features were used to operationalise this cognitive skill. Specifically, the first measure used was disproportionately poor performance on phonological related distractor-target pairs in picture-word verification (relative to semantic judgements). The premise behind the subtraction of semantic judgments in this manipulation is to eliminate the mapping of the recognised word onto its meaning. This ensures we are selectively assessing the auditory recognition component of the task. However, it must be noted that repetition does involve other cognitive process unrelated to auditory word recognition. These include mapping the heard word onto it's appropriate meaning, and selecting the item within the mental lexicon for subsequent production (see dual-route model of repetition: McCarthy and Warrington (1984)). Consequently, we additionally compared performance on repetition to picture naming in order to partial out these potential other cognitive skills that are involved in this task that could confound performance in repetition. This ensures that we are specifically assessing the auditory word recognition component of the task. 


\section{Verb Retrieval}

Researchers have argued that the cognitive processes underlying verb retrieval are unique from other linguistic capabilities (see Black \& Chiat 2003). It is therefore important that this cognitive skill is individually assessed by the BLAST. To operationalise verb retrieval we used the verb generation task, as manipulations within this task are used to assess other cognitive skills and we aimed to adhere to a brief administration time. A possible concern with using the verb generation task to assess this cognitive skill is that it may not be as pure a measure of verb retrieval as the commonly used action-naming task. To produce a verb associated with a concrete noun, one must also access the meaning of the noun (for discussion see Martin \& Cheng, 2006). Another problems is that there is no carefully matched object naming task with which to compare to. Consequently, for the operationalization of verb retrieval using the BLAST we compared patients' performance in verb generation standardised to a $\mathrm{z}$ score, to their performance in picture naming, also standardised. This ensures that we are eliminating cognitive skills that are associated with both tasks, and therefore specifically assessing the demands of verb retrieval. We also only used items with a strong verb associate (high selection condition) to further eliminate the possible implications that deficits in resolving competition could have on overall verb generation performance.

\section{Goal-Driven Response Selection}

The patient group used to operationalise the cognitive skill goal-driven response selection, was patients with nonfluent aphasia. It has been proposed that these patients may have a deficit in a control system that manages competition between conflicting representations (Biegler, Crowther, \& Martin, 2008; Hamilton \& Martin, 2005; January, Trueswell, \& Thompson-Schill, 2009; Robinson, Shallice, Bozzali \& Cipolotti, 2010; Schnur et al., 2006; Thompson-Schill, D’Esposito, Aguirre \& Farah, 1997, Thompson-Schill, 
D’Esposito, \& Kan 1999; Wilshire \& McCarthy, 2002). Patients with nonfluent aphasia tend to perform disproportionately poorly in tasks that appear to involve selecting amongst a number of alternative responses, or coming up with a single verbal response when many alternatives are possible (Cameron-Jones 2008; Hamilton \& Martin, 2005; Robinson, Shallice, Bozzali \& Cipolotti 2010; Scott \& Wilshire, 2010). For example, they are extremely slow to response to incongruent items in the Stroop task (Scott \& Wilshire, 2010; CameronJones, 2008), and they also perform disproportionately poorly in tasks when the stimulus affords numerous responses (e.g., rope -> “tie", "knot”, "pull”, “drag”) (Thompson-Schill, D’Esposito, Aguirre, \& Farah, 1997; Thompson-Schill, D’Esposito, \& Kan, 1999). As noted above our measure of goal-driven response selection therefore included both the Stroop and the verb generation tasks there is a debate in the literature as to whether the effects seen in verb generation reflect a difficulty selecting amongst competing responses or whether they reflect a difficulty with more effortful search through the lexicon (see especially Martin \& Cheng, 2006). We remain neutral on this debate by referring to the skill as "goal-driven response selection."

The third task/manipulation we used for the operationalization of goal driven response selection is directed primarily at the more general idea of internal goal-driven responding. We selected letter fluency to assess this. For successful completion of this task one must engage in appropriate lexical search through one's mental lexicon in order to select items that adhere to the goal of the task i.e. searching for words that begin with the letter "A" (for review see Henry \& Crawford, 2004).

\section{Articulatory-Motor Planning}

The patient group used to operationalise this cognitive skill was patients with apraxia of speech, as it has been proposed that these patients have a specific deficit in in the ability to coordinate speech movements (Frey et al., 1987; Johns \& Darley, 1970). These patients 
consistently demonstrate poor performance in articulatory agility tasks (e.g. Dronkers, 1996). To operationalise articulatory-motor planning we used a common measure of verbal agility derived from the Boston Diagnostic Aphasic Examination (BDAE: Goodglass, Kaplan and Barresi, 2001). In this task, participants repeat a verbal sequence as many times as possible within five seconds. The premise behind this task is that the repetition of the same lexical item over and over again eliminates the demands on other cognitive skills such as phonological encoding and lexical selection. Consequently, the motor demands placed on the articulators are necessary for successful performance on this task.

\section{Supplementary Cognitive Skills}

The tasks used to assess the following cognitive skills were added to the BLAST halfway through the testing phase, and scores were only derived for a subset of patients. Consequently, these skills have been referred to collectively as supplementary cognitive skills.

\section{Verbal Short-Term Memory}

It has been consistently demonstrated that patients with deficits in verbal short-term memory - identified by having a poor digit span - have similarly poor performance in nonword repetition (see esp. Gathercole \& Baddeley, 1989, 1993). As a result of such findings, we operationalised this cognitive skill by calculating accuracy in a nonword repetition task.

\section{Reading Skills: Visual Word Recognition, Orthographic-Phonological Mapping, and Semantic-Phonological Mapping}

The patient groups used to operationalise the cognitive skills involved in reading (visual word recognition, orthographic-phonological mapping and orthographic-semantic mapping) was patients with specific reading disorders (pure alexia, phonological dyslexia, surface dyslexia). It has been argued that patients with pure alexia have a deficit in visual 
word recognition (e.g., Farah \& Wallace, 1991). Most pure alexic patients retain the ability to identify single letters, but adopt a letter-by-letter strategy when reading. Consequently, patients with pure alexia usually show a linear relationship between the number of letters in a word and the time taken to read it (see esp. Behrmann, Black \& Bub, 1990). Therefore the manipulation we have used for the operationalisation of visual word recognition was word length (effect of number of letters on reading time) effect in single word reading.

In contrast, phonological dyslexia is a disorder that reflects a selective breakdown in the sublexical cognitive processes associated with reading, and specifically the graphemephoneme conversion mechanism (Coltheart et al., 2001; Ellis, 1980; Patterson \& Shewell, 1987; Shallice, 1988). Patients with phonological dyslexia consistently produce disproportionate errors in non-word reading compared to read words (Beauvois \& Derouesne, 1979; Coltheart 1996; Henry, Beeson, Stark, \& Rapcsak, 2007; Roeltgen, Sevush \& Heilman, 1983; Shallice, 1981). Therefore, the manipulation we have used to operationalise orthographical-phonological mapping is performance in a nonword reading task.

Finally, it has been argued that patients with surface dyslexia have a specific deficit in the mapping from orthography to semantics (Coltheart, et al., 2001; Jobard, Crivello, Tzourio-Mazoyer, 2003). Patients with surface dyslexia consistently produce more errors in reading irregular words, compared to regular words (e.g., Behrmann \& Bub, 1992; McCarthy $\&$ Warrington, 1984). Therefore, the manipulation we used to operationalise orthographicalsemantic mapping is accuracy in reading irregular words relative to regular words. 
Table 2.2

Task profiles used to operationalise the core cognitive skills assessed by the BLAST

Core Skill

Accessing Semantic Knowledge

Phonological Encoding

Auditory Word Recognition

Goal-Driven Response Selection

Verb Retrieval

Articulatory-Motor Planning

Verbal STM

Visual Word Recognition

Orthographic-Phonological

Mapping

\section{BLAST Task Profile}

Semantic Confusions in Picture-Word Verification

Verbal Fluency: poorer category than letter

fluency

Production of Semantic Errors in Picture Naming

Strong frequency effect in Picture Naming

Production of Omission Errors in Picture Naming

Production of Semantic Errors in Picture Naming

Normal Picture Word Verification

Strong Length Effects in Picture Naming

Abnormal Latency in Repetition and Nonword

Repetition

Production of Phonological Errors in Picture

Naming

Normal Articulatory Agility

Phonological Confusions in Picture Word

Verification

Abnormal latency in Repetition

Poor Repetition accuracy relative to Picture

Naming accuracy

Abnormal Selection Effect in Verb Generation

Abnormal Congruency Effect in Stroop

Poor Letter Fluency

Poor Verb Generation in Low Selection Items

Relative to Picture Naming

Poor Articulatory Agility Score

Poor Nonword Repetition

Strong Length Effect in Single Word Reading

Poor Nonword Reading 


\begin{tabular}{cc}
\hline Core Skill & BLAST Task Profile \\
\hline Orthographic-Semantic & Poor Reading of Irregular Words Relative to \\
Mapping & Regular Words \\
\hline
\end{tabular}

\section{Aims and Hypotheses}

In this thesis, we examine the performance of a sample of 40 undifferentiated tumour patients on the BLAST preoperatively (the day before surgery) and postoperatively (two to three days after surgery). The first aim of the study is to explore the incidence of language impairments in our tumour population, both in terms of the overall task performance, and in terms of the 11 core cognitive skills. We examine overall performance of each tumour patient, on each of the eight tasks that make by the BLAST. We will explore the overall percentage of impairment, as well as the incidence of impairment on each task and differences between pre and postoperative performance. Then, based on the approach summarised in Table 2.2, we will create a measure for each patient for each of the 11 core cognitive skills assessed by the BLAST. Three aspects of participants' scores will then be examined:

1. Overall percentage of patients scoring significantly below controls on at least one cognitive skill (overall sensitivity).

2. The relative incidence of impairments across each cognitive skills (selectivity)

3. Any differences in the above measures between preoperative and postoperative assessment phase

The second aim of the study is to explore the relationships between lesion location and other lesion characteristics with our language outcome measures, particularly, those related to the key cognitive skills. To do this, we will first perform some simple group comparisons to examine whether individuals with tumours to different broad brain regions (right frontal, left frontal, right posterior, left posterior) and different tumour pathologies show reliably different cognitive skill profiles. Our specific hypotheses here are as follows: 
1. That average scores for those cognitive skills in the anterior group - namely, articulatory motor planning, goal driven response selection and verb retrieval (as operationalised in Table 2.2) will be significantly lower in the left frontal group compared to those with a tumour in other anatomical regions, including the left posterior group.

2. That the average scores for those cognitive skill in the posterior group - namely, phonological encoding, lexical selection, accessing semantic knowledge, and auditory word recognition (as operationalised in Table 2.2) will be significantly lower in the left posterior group compared to those with a tumour in other anatomical regions, including the left anterior group.

We then perform a finer-grained analysis of the relationship between core skills and lesion locations using Voxel Symptom Mapping (Bates et al., 2003). Based on our review of the literature, we derived a series of more specific hypotheses in regards to the core cognitive skills examined by the BLAST, and their neural underpinnings, which are outlined in Table 2.3. The predictions will be primarily based on preoperative performance, but postoperative performance will also be explored. In Table 2.3 the hypotheses pertaining to reading and verbal short-term memory are in parentheses; these are to be regarded as tentative, exploratory hypotheses only, due to the small number of patients who completed the relevant tasks.

Table 2.3

Predictions derived for specific brain regions that significantly predict performance in each core cognitive skill.

Cognitive Skill Accessing Semantic Knowledge Lexical Selection
Brain Region

Left anterior temporal regions

Left posterior temporal region 


\section{Cognitive Skill}

Auditory Word Recognition

Goal Driven Response Selection

Verb retrieval

\section{Brain Region}

Left posterior superior temporal lobe

Left inferior frontal gyrus

Left inferior frontal regions
Articulatory Motor Planning.

(Verbal Short-Term Memory)

(Visual Word Recognition)
Left inferior frontal regions, including the left insula

(left inferior parietal cortex)

(left fusiform gyrus)

(Orthographical-Phonological Mapping) (left posterior temporo-parietal regions, and the left inferior frontal gyrus)

(Orthographical-Semantic Mapping)

(left posterior temporal regions) 


\section{Chapter 3: Method}

\section{Participants}

Tumour Participants. 40 patients from the Neurosurgical Ward of Wellington Hospital, New Zealand participated in this study from December 2011 to December 2013. 19 patients were male and 21 were female, with a mean age of 54.40 (range 30-78, SD 13.28). The inclusion criterion for recruitment was a cerebral tumour requiring craniotomy for debulking or complete resection, irrespective of aetiology, location, and malignancy (see Appendix A for a brief case description and MRI scan of each patient tested in the current study). Participants were excluded if they had any prominent visual disturbances, English was not their native tongue, or had a cerebellar tumour. Of the 40 patients tested preoperatively, seven patients did not complete postoperative testing due to one of the following: early discharge (four), postoperative complications (two), or declined (one). Patient clinical and demographic data is reported in Table 3.2. Patient testing was approved by the New Zealand Health and Disability Ethics Committee (reference number: CEN/11/07/037).

Table 3.1 gives a breakdown of the number of patients according to broad lesion sites based on presurgical radiology reports. It is important to note that the left posterior and right posterior groups contained a very small number of patients. Any inferences made about these groups should be tentative. Two patients were not categorized into a group due to their multiple tumour presentation, and were therefore excluded from group analysis. Lesion overlap maps for the four groups based on normalised MRI images of their lesions are presented in Figures 3.1 to 3.4. Each patient's MRI scan was normalized using methodology described in Chapter 6. 
Table 3.1

The number of patients in each anatomical group pre and postoperatively based on presurgical radiology reports

\begin{tabular}{ccc}
\hline Anatomical Group & Preoperative & Postoperative \\
\hline Left Frontal & 18 & 14 \\
Left Posterior & 5 & 4 \\
Right Frontal & 10 & 9 \\
Right Posterior & 5 & 4 \\
\hline
\end{tabular}

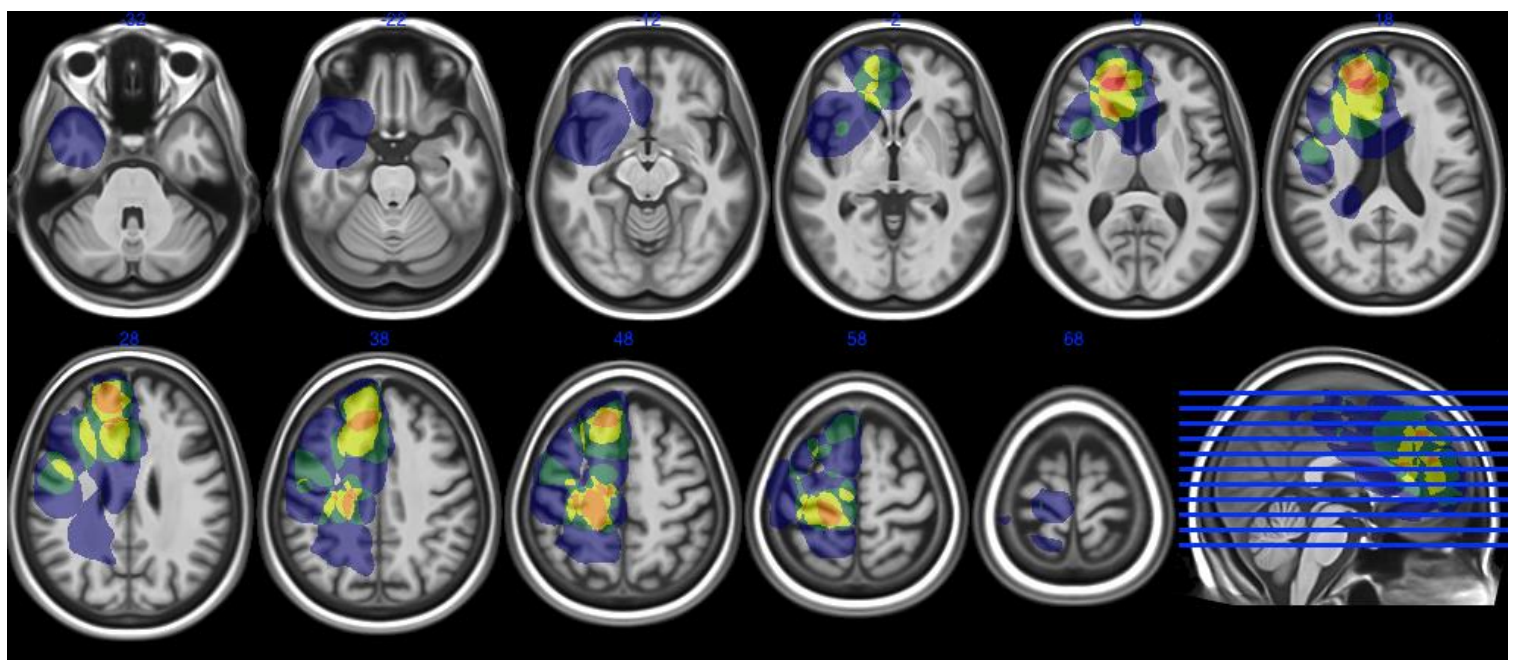

Figure 3.1. Lesion overlay map for individuals in the left frontal group ( $=18)$. Region showing axial slices on a standard template (Rorden, Bonilha, Fridriksson, Bender \& Karnath, 2012) at MNI Coordinates $=-22,-12,-2,3,8,19,28,38,48,58,68$. Red $=$ overlap between five individuals; orange $=$ overlap between four individuals; yellow $=$ overlap between three individuals; green = overlap between two individuals; blue = no overlap, i.e., lesion is confined to one individual. Further details of imaging methods and image preparation are described in Chapter 6. 


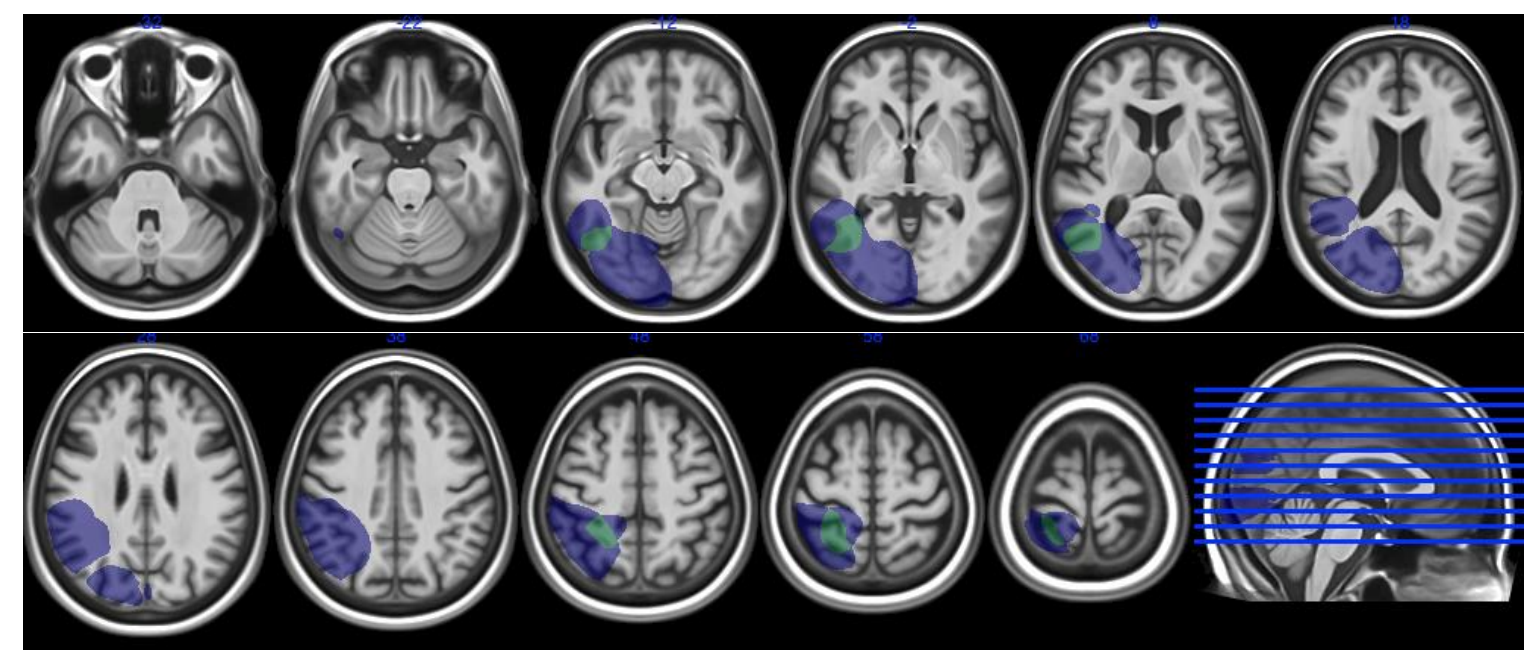

Figure 3.2. Lesion overlay map for individuals in the left posterior group. ( $\mathrm{N}=4$; note: one patient's MRI scan was unavailable). Region showing axial slices on a standard template (Rorden et al., 2012) at MNI Coordinates $=-22,-12,-2,3,8,19,28,38,48,58,68$. Green $=$ overlap between two individuals; blue $=$ no overlap, i.e., lesion is confined to one individual . Further details of imaging methods and image preparation are described in Chapter 6.

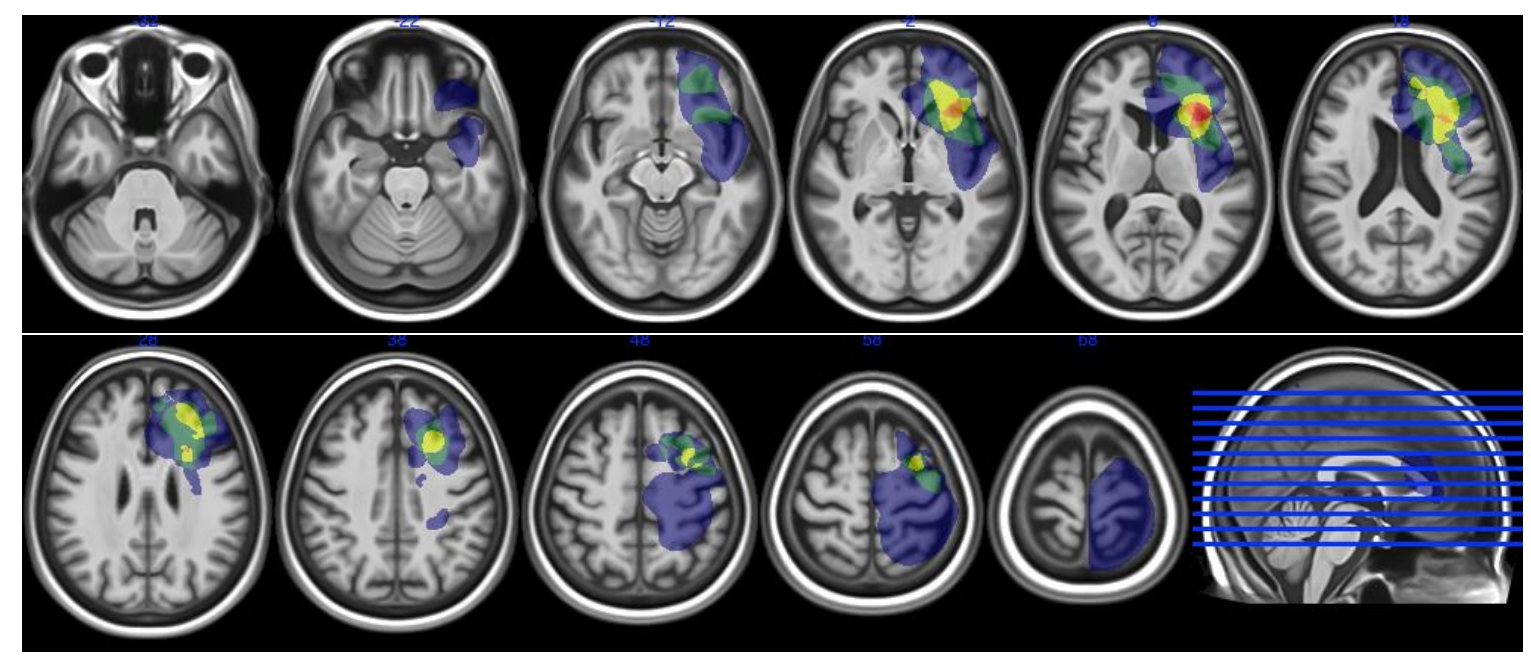

Figure 3.3. Lesion overlay map for individuals in the right frontal group ( $\mathrm{N}=9$; one patient's MRI scan was unavailable). Region showing axial slices on a standard template (Rorden et al., 2012) at MNI Coordinates $=-22,-12,-2,3,8,19,28,38,48,58,68$. Red $=$ overlap between five individuals; orange $=$ overlap between four individuals; yellow = overlap between three individuals; green = overlap between two individuals; blue = no overlap, i.e., 
lesion is confined to one individual. Further details of imaging methods and image preparation are described in Chapter 6.

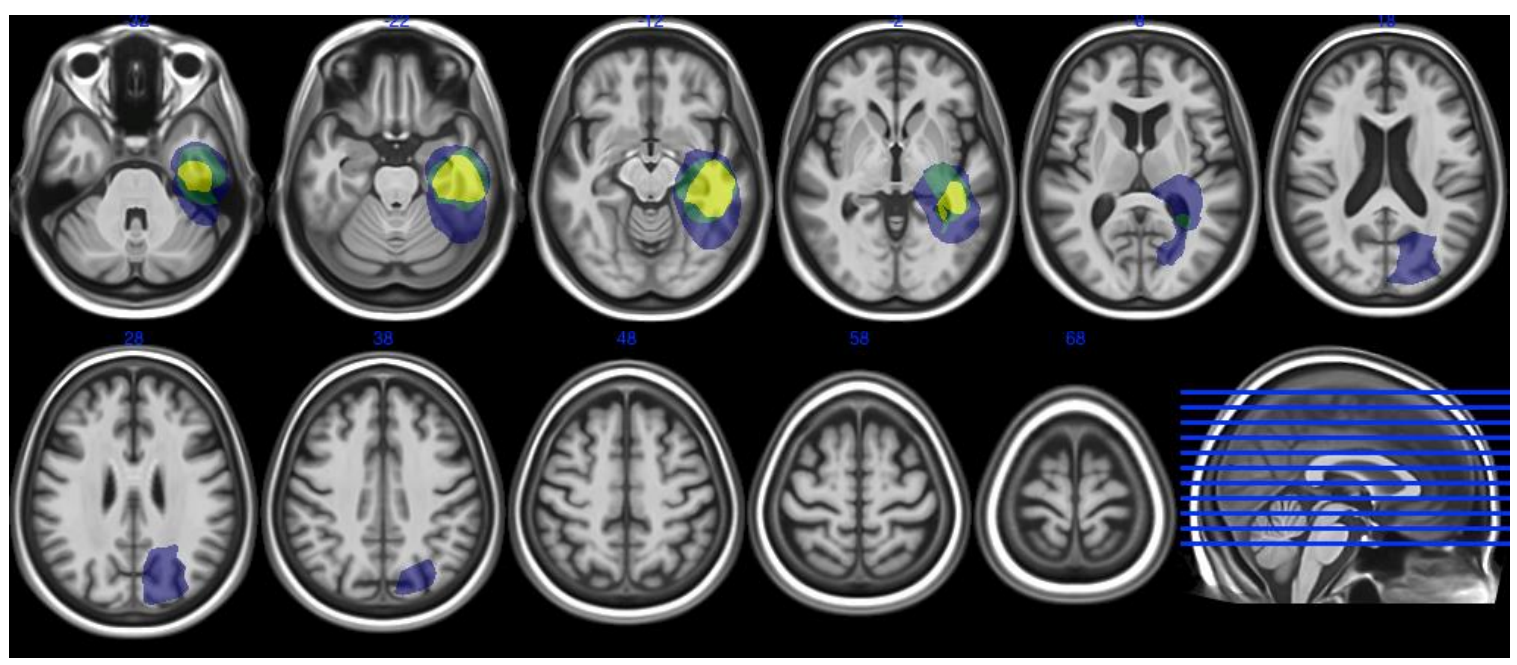

Figure 3.4: Lesion overlay map for individuals in the right posterior group ( $\mathrm{N}=4$; one patient's MRI scan was unavailable). Region showing axial slices on a standard template (Rorden et al., 2012) at MNI Coordinates $=-22,-12,-2,3,8,19,28,38,48,58,68$. Yellow $=$ overlap between three individuals; green $=$ overlap between two individuals; blue $=$ no overlap, i.e. lesion is confined to one individual. Further details of imaging methods and image preparation are described in Chapter 6 .

Healthy Controls. In addition to the brain tumour patients, 60 healthy controls also completed the testing protocol. Controls were recruited via three methods: i) through the Victoria University Psychology IPRP programme (this programme allows first year psychology students to gain course credit for completing psychological experiments); ii) through a pre-existing register of healthy controls that had indicated their willingness to be contacted about future studies in the Neuropsychology Laboratory at Victoria University; and iii) through community advertising. Testing occurred either at the Victoria University Psychology Department or the participant's home. Participants who were recruited through the Victoria University Psychology IPRP programme received course credit. All other 
participants received monetary compensation: participants that travelled to the university received $\$ 20$, and those visited by the experimenter received $\$ 10$ (money was available through a grant received by the Neurological Foundation of New Zealand). Before participating, participants were required to read through an information sheet and sign a consent form (see Appendix B), and then complete a Neurological Status Questionnaire (see Appendix C). If the participant reported any neurological injury, they were informed that they did not meet the criteria to participate in this study, and were thanked for their time.

These individuals were then organised into three subgroups comprising 20 participants each. The "young” group consisted of participants aged between 18 and 29 (mean 22.35), and comprised 10 males and 10 females. All were first year psychology students who participated in return for course credit. As there were no patients in the current study within this age group, this group did not act as controls for any of the patient reported here. The "intermediate" group were aged between 30 and 50 (mean age 37.85) and comprised 7 males and 13 females. 10 of these participants were first year psychology students, who completed the study in return for course credit; the remainder were recruited through community advertising and received $\$ 10$ (or $\$ 20$ if they had travelled to Victoria University for testing) as compensation for taking part. These individuals served as a control group for patients in the $30-50$ age group, whose mean age was 40.63 ; these two groups did not differ significantly in age $(t(34)=-1.384, \mathrm{p}=.175)$. And finally, the "mature" group consisted of participants aged 51 years or more (mean age 68.9), and comprised seven males and 13 females. They were recruited through community advertising, and reimbursed $\$ 10$ (or \$20 if they had travelled to Victoria University for testing). These participants served as a control group for patients in the $51+$ age group, whose mean age was 65.58 ; these two groups did not differ significantly in age $(t(42)=1.939, \mathrm{p}=.059)$. The inclusion criterion for participation was no reported history of neurological injury or disease, measured by 
completion of a brief neurological status questionnaire. Participants were also excluded if they had a visual impairment and English was not their native tongue. The testing of healthy controls was approved by the Victoria University School of Psychology Ethics Committee (reference number: RM019226). 
Table 3.2

The demographic and clinical information of each patient who completed the BLAST

\begin{tabular}{|c|c|c|c|c|c|c|c|c|}
\hline $\begin{array}{l}\text { Patient (51+ } \\
\text { Group) }\end{array}$ & Age & Gender & $\begin{array}{l}\text { Tumour } \\
\text { Specimen }\end{array}$ & $\begin{array}{c}\text { Anatomical } \\
\text { Group }\end{array}$ & $\begin{array}{c}\text { Tumour } \\
\text { Volume }\left(\mathrm{cm}^{3}\right)\end{array}$ & Oedema & Surgery Type & $\begin{array}{c}\text { Pre and } \\
\text { Postoperative } \\
\text { Testing }\end{array}$ \\
\hline SM & 51 & $\mathrm{~F}$ & $\begin{array}{c}\text { Cavernous } \\
\text { haemangioma - } \\
\text { Cavernoma }\end{array}$ & L Frontal & 1.9 & No & $\begin{array}{c}\text { Awake craniotomy and } \\
\text { resection }\end{array}$ & Yes \\
\hline VD & 56 & $\mathrm{~F}$ & Glioblastoma & L Frontal & 20.36 & No & $\begin{array}{c}\text { Craniotomy for debulking } \\
\text { and left frontal lesion }\end{array}$ & Yes \\
\hline $\mathrm{RF}$ & 65 & M & Meningioma & L Frontal & 17.5 & Yes & Craniotomy and removal & Yes \\
\hline SO & 58 & M & Glioblastoma & L Frontal & 15.02 & No & Craniotomy and debulking & Yes \\
\hline EMH & 75 & $\mathrm{~F}$ & $\begin{array}{l}\text { Unknown but } \\
\text { metastatic }\end{array}$ & L Frontal & 7.36 & Yes & Craniotomy and resection & Only Preoperative \\
\hline EA & 70 & $\mathrm{~F}$ & Meningioma & L Frontal & 14.64 & No & Craniotomy and resection & Yes \\
\hline $\mathrm{JM}$ & 71 & $\mathrm{~F}$ & $\begin{array}{l}\text { Metastatic with } \\
\text { oedema }\end{array}$ & L Frontal & 3.55 & Yes & $\begin{array}{c}\text { Craniotomy and } \\
\text { exploration }\end{array}$ & Yes \\
\hline $\mathrm{CR}$ & 75 & $\mathrm{~F}$ & Meningioma & L Frontal & 51.57 & No & Craniotomy and removal & Yes \\
\hline $\mathrm{BCA}$ & 56 & M & $\begin{array}{c}\text { Recurrent } \\
\text { glioblastoma }\end{array}$ & L Posterior & 86.86 & Yes & Craniotomy and resection & Yes \\
\hline $\mathrm{BD}$ & 62 & $\mathrm{~F}$ & Meningioma & L Posterior & 35.97 & No & Craniotomy and resection & Yes \\
\hline LA & 63 & $\mathrm{~F}$ & Glioblastoma & R Frontal & 3.89 & na & Craniotomy and resection & Yes \\
\hline $\mathrm{CM}$ & 57 & M & Oligodendroglioma & R Frontal & 1.67 & Yes & Craniotomy and excision & Yes \\
\hline LW & 66 & $\mathrm{~F}$ & Meningioma & R Frontal & 6.4 & Yes & Craniotomy and resection & Yes \\
\hline ES & 78 & M & Glioblastoma & R Frontal & 43.75 & Yes & $\begin{array}{c}\text { Craniotomy and subtotal } \\
\text { resection }\end{array}$ & Yes \\
\hline
\end{tabular}




\begin{tabular}{|c|c|c|c|c|c|c|c|c|}
\hline $\begin{array}{l}\text { Patient (51+ } \\
\text { Group) }\end{array}$ & Age & Gender & $\begin{array}{l}\text { Tumour } \\
\text { Specimen }\end{array}$ & $\begin{array}{l}\text { Anatomical } \\
\text { Group }\end{array}$ & $\begin{array}{c}\text { Tumour } \\
\text { Volume }\left(\mathrm{cm}^{3}\right)\end{array}$ & Oedema & Surgery Type & $\begin{array}{c}\text { Pre and } \\
\text { Postoperative } \\
\text { Testing }\end{array}$ \\
\hline $\mathrm{RG}$ & 58 & $\mathrm{M}$ & Gliosarcoma & R Posterior & 27.87 & na & Craniotomy and debulking & Yes \\
\hline TKH & 60 & M & Glioblastoma & R Posterior & 47.9 & No & Craniotomy and debulking & Yes \\
\hline JAS & 55 & M & Glioma & R Posterior & 75.25 & Yes & Craniotomy and resection & Only preoperative \\
\hline AEK & 52 & $\mathrm{~F}$ & Glioma & R Posterior & 39.66 & Yes & Craniotomy and resection & Yes \\
\hline PAJ & 65 & M & Glioblastoma & $\begin{array}{l}\text { R Frontal \& L } \\
\text { Posterior }\end{array}$ & na & No & $\begin{array}{l}\text { Craniotomy and resection } \\
\text { of } \mathrm{R} \text { posterior lesion }\end{array}$ & Yes \\
\hline SMC & 63 & M & Glioblastoma & R Frontal & 63.29 & No & Craniotomy and removal & Yes \\
\hline MRO & 60 & M & Meningioma & R Frontal & 90.51 & No & Craniotomy and removal & Yes \\
\hline $\mathrm{RJ}$ & 64 & $\mathrm{~F}$ & $\begin{array}{c}\text { Metastatic tumour } \\
\text { from right lung } \\
\text { mass }\end{array}$ & L Posterior & 14.74 & Yes & No Surgery & Only Preoperative \\
\hline GP & 73 & M & Meningioma & R Frontal & 56.98 & Yes & Craniotomy and resection & Yes \\
\hline MR & 68 & $\mathrm{~F}$ & $\begin{array}{l}\text { Metastatic } \\
\text { melanoma }\end{array}$ & L Frontal & 19.24 & Yes & $\begin{array}{l}\text { Craniotomy for resection } \\
\text { of left frontal lesion }\end{array}$ & Only Preoperative \\
\hline $\begin{array}{l}\text { Patient }(30-50 \\
\text { Group) }\end{array}$ & Age & Gender & $\begin{array}{l}\text { Tumour } \\
\text { Specimen }\end{array}$ & $\begin{array}{l}\text { Anatomical } \\
\text { Group }\end{array}$ & $\begin{array}{c}\text { Tumour } \\
\text { Volume }\left(\mathrm{cm}^{3}\right)\end{array}$ & Oedema & Surgery Type & $\begin{array}{c}\text { Pre and } \\
\text { Postoperative } \\
\text { Testing }\end{array}$ \\
\hline PM & 45 & M & $\begin{array}{l}\text { Astrocytoma with } \\
\text { possible } \\
\text { oligodendroglioma } \\
\text { component }\end{array}$ & L Frontal & 0.72 & Yes & $\begin{array}{c}\text { Awake craniotomy and } \\
\text { debulking }\end{array}$ & Yes \\
\hline DA & 33 & M & Meningioma & L Frontal & 30.69 & No & Craniotomy and resection & Yes \\
\hline $\mathrm{EH}$ & 39 & $\mathrm{~F}$ & Meningioma & L Frontal & 26.77 & Yes & Craniotomy and resection & Yes \\
\hline
\end{tabular}




\begin{tabular}{|c|c|c|c|c|c|c|c|c|}
\hline $\begin{array}{l}\text { Patient (30-50 } \\
\text { Group) }\end{array}$ & Age & Gender & $\begin{array}{l}\text { Tumour } \\
\text { Specimen }\end{array}$ & $\begin{array}{l}\text { Anatomical } \\
\text { Group }\end{array}$ & $\begin{array}{c}\text { Tumour } \\
\text { Volume }\left(\mathrm{cm}^{3}\right)\end{array}$ & Oedema & Surgery Type & $\begin{array}{c}\text { Pre and } \\
\text { Postoperative } \\
\text { Testing }\end{array}$ \\
\hline $\mathrm{LC}$ & 43 & $\mathrm{~F}$ & Astrocytoma & L Frontal & 46.75 & No & $\begin{array}{c}\text { Awake craniotomy and } \\
\text { resection }\end{array}$ & Yes \\
\hline $\mathrm{CA}$ & 47 & $\mathrm{~F}$ & Meningioma & L Frontal & 83.57 & Yes & Craniotomy and resection & Yes \\
\hline $\mathrm{CG}$ & 46 & $\mathrm{~F}$ & na & L Frontal & 58.92 & No & Craniotomy and resection & Yes \\
\hline AVG & 42 & $\mathrm{~F}$ & Meningioma & L Posterior & 71.33 & No & Craniotomy and resection & Yes \\
\hline $\mathrm{AM}$ & 38 & $\mathrm{~F}$ & Glioblastoma & L Posterior & 28.8 & Yes & Craniotomy and resection & Yes \\
\hline $\mathrm{JB}$ & 47 & $\mathrm{~F}$ & Epidermoid lesion & R Posterior & 41.11 & na & Craniotomy and resection & Yes \\
\hline $\mathrm{AE}$ & 46 & M & Haemangioma & $\begin{array}{c}\text { L Frontal \& R } \\
\quad \text { Frontal }\end{array}$ & na & No & $\begin{array}{c}\text { Craniotomy and removal } \\
\text { of left frontal lesion }\end{array}$ & Yes \\
\hline TT & 36 & $\mathrm{~F}$ & $\begin{array}{l}\text { Low-grade } \\
\text { astrocytoma }\end{array}$ & R Frontal & 11.35 & No & Craniotomy and resection & Yes \\
\hline $\mathrm{KB}$ & 30 & $\mathrm{~F}$ & Meningioma & R Frontal & na & na & Craniotomy and resection & Only Preoperative \\
\hline DAP & 45 & M & Astrocytoma & L Frontal & 31.25 & Yes & Craniotomy and resection & Only Preoperative \\
\hline $\mathrm{TF}$ & 43 & $\mathrm{~F}$ & Glioblastoma & L Frontal & 49.08 & Yes & Craniotomy and debulking & Yes \\
\hline $\mathrm{TD}$ & 30 & M & Oligodendroglioma & L Frontal & 151.59 & No & Craniotomy and debulking & Only Preoperative \\
\hline $\mathrm{DF}$ & 40 & M & Glioma & R Frontal & 50.36 & No & Craniotomy and removal & Yes \\
\hline
\end{tabular}




\section{The BLAST: Brief Language Assessment for Surgical Tumours}

The BLAST comprises the eight tasks outlined in Table 2.1. Two versions of the test battery were created, so as to minimise any carryover effects between preoperative and postoperative assessment. Below is a detailed description of each task. In addition, a comprehensive list of all stimuli contained in each task and its subsequent manipulation is presented in Appendix D.

\section{Picture Naming.}

In this task, participants are required to produce the name of a visually displayed object. The to-be-produced items varied in both frequency rating and syllable length.

1.1 Materials. This task consisted of 120 items drawn from the New Zealand Length by Frequency Naming Test (Wilshire, 2002). Pictures in this test produced at least $80 \%$ name agreement on a previous pilot study which involved 70 normal speakers of varying ages and backgrounds. This subset was selected from a pool of 180 items because they varied most appropriately in CELEX lemma frequency ratings (Baayen, Piepenbrock \& van Rijn, 1993) and length in syllables. More specifically, 40 of the items selected depict a low frequency noun (with frequency ratings of less than 70; range 4-69, mean 34.92), 40 depict a medium frequency noun (with frequency ratings between 70-200; range 72-199; mean 129.32), and the remaining 40 depict a high frequency noun (with frequency ratings of 200 or more; range 205-2441; mean 763.00). Further, each of these frequency groups comprised approximately equal numbers of monosyllabic, bisyllabic and polysyllabic items, thus creating nine different frequency $\mathrm{x}$ length combinations. Both frequency and length were balanced so that there were no systematic frequency differences between the monosyllabic, bisyllabic and polysyllabic items, and similarly, no length differences between the low, medium and high frequency words (for more information, see Wilshire, 2002). Items in each of the nine frequency $\mathrm{x}$ length combinations were then allocated to one of two versions of the task. Each 
version consisted of 60 items with 20 items each for the low, medium and high frequency manipulations; and 20 each for the mono, bi and polysyllabic manipulations. A one-way ANOVA of the log CELEX lemma frequency values (Baayen, Piepenbrock \& van Rijn, 1993) of each item confirmed that there was no significant difference between the high frequency $(F(2,58)=.162, p=.860)$, medium frequency $(F(2,58)=0.52, p=.608)$, and low frequency $(F(2,58)=4.37, p=.648)$ conditions between the two test versions (see Table 3.3).

Table 3.3

The mean log CELEX lemma frequencies for the two versions of the picture naming task Mean CELEX Lemma Frequency

\begin{tabular}{cccc} 
Version & High & Medium & Low \\
\hline 1 & 776.25 & 128.82 & 37.15 \\
2 & 758.58 & 134.90 & 40.74 \\
\hline
\end{tabular}

1.2 Procedure In this task, each individual picture is presented on a laptop screen and the participant must produce its name. For each version of the task, a practice picture was presented first, so participants were familiarized with the objectives of task. The 60 experimental items were then presented in a fixed pseudo random order. Before each picture, a fixation cross-appeared for 100ms (see Figure 3.5) followed by the presentation of a picture and a tone simultaneously. The tone acted as a marker, facilitating measurement of response latency. Participants were given unlimited time to make a response, and if an incorrect response was made, no feedback was given. Picture presentation was self-paced; the researcher initiated the presentation of the next fixation cross with a key press, after a response had been made. 


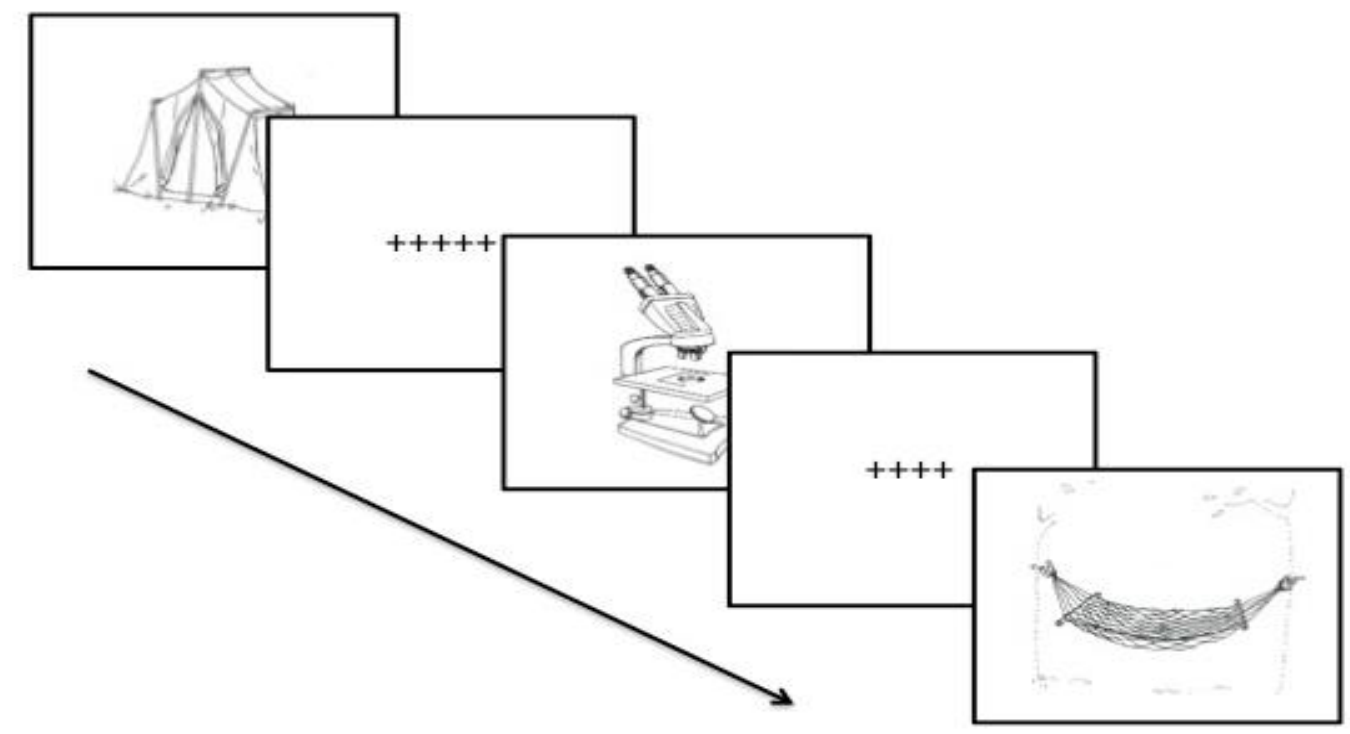

Figure 3.5. Item presentation on a laptop computer for the picture naming task

\subsection{Response Scoring}

1.3.1 Accuracy. Responses for each target item were scored as correct or incorrect. Only the first response made by the participant was scored; if the participant's first response was incorrect but was then changed to a correct response, this was still recorded as incorrect. Alternative names for an item, e.g., "spaceman" for the item "astronaut" were also scored as incorrect. Incorrect responses were further categorised by error type according to the scheme shown in Table 3.4.

The total number of correct responses for the entire test was calculated and converted to a percentage. The overall percentage of correct responses for each frequency and length manipulation was then calculated. This was then used to calculate the slope of the length and frequency effects using the slope function in EXCEL. For example, if a participant scored low frequency $=80 \%$, medium frequency $=90 \%$, high frequency $=100 \%$ on the frequency manipulation, the frequency effect would the size of the slope of these scores, which is 10. 
Table 3.4

Types of errors coded for in the picture-naming task

\begin{tabular}{|c|c|c|}
\hline Error Type & Definition & Example \\
\hline Phonological & $\begin{array}{l}\text { A real word that is phonologically } \\
\text { related to the target by above definition }\end{array}$ & boat $->$ bake \\
\hline Semantic & $\begin{array}{l}\text { A real word that is semantically related } \\
\text { to the target. Should be from same } \\
\text { category (e.g., apple, banana). } \\
\text { Associates (apple-core) don't count }\end{array}$ & boat $->$ car \\
\hline Mixed & $\begin{array}{l}\text { A real word that is phonologically and } \\
\text { semantically related to the target. }\end{array}$ & carrot $->$ cabbage \\
\hline Unrelated & $\begin{array}{l}\text { A real word that is not phonologically } \\
\text { or semantically related to the target }\end{array}$ & boat $->$ rice \\
\hline Omission & No direct attempt at word & \\
\hline Alternative & $\begin{array}{l}\text { A word that is entirely appropriate, but } \\
\text { not the target }\end{array}$ & couch $->$ sofa \\
\hline Other Error & Not classifiable as any other error. & \\
\hline
\end{tabular}

1.3.2 Response Latency. Response latencies for each correct response were also calculated using audacity software (Audacity Team, 2008). Latencies were recorded from the start of each tone, to the start of the participant's first response. Fillers such as "um" and "er" were ignored. However if an article or other modifier was produced before the noun (e.g. "a tent"), the response latency was measured from the onset of the modifier. The latency data was trimmed of outliers (exceedingly long latencies) using a two-step method. First, the data was winsorized: the longest response latency was replaced by the second longest response latency, and then the second longest response latency replaced by the third longest. Second, 
any response latencies that lay two and a half standard deviations from the winsorized mean was removed. Once this had been done, the average response time for the entire task was calculated, as well as for each frequency and length manipulation. Frequency and length effects were calculated in the same manner outlined in section 1.3.1.

\section{Verb Generation}

In the verb generation task, participants must produce an action word that is associated with a visually displayed concrete noun.

2.1 Materials. The verb generation task used was adapted from a task originally devised by Cameron Jones (2008). This task comprises 90 picturable nouns, each of which is used as a stimulus to elicit a verb describing an action associated with that item (e.g., scissors -> "cut"). In this task, half of the 90 items had low selection demands; that is, based on previous pilot testing, the commonly elicited one specific verb (the most common response was given at least five times more often than the next most popular verb response in a pilot study involving normal participants). The remaining half of the items had high selection demands (the most commonly elicited verb response was given no more than three times more often than the next most popular) (see Cameron Jones, 2008). These two groups of items will be referred to as low and high selection demands respectively. In both the high and the low selection groups, there were equal numbers of high and low frequency stimulus nouns (defined as having CELEX lemma frequency of more than 450 or less than 450 , respectively).

In our adaptation, we created two versions of the test, each containing 45 items. Version one included 22 high selection items and 23 low selection items, and in each condition, there were roughly equal numbers of low and high frequency items. Version two included 23 high selection items and 22 low selection items, again, with each condition containing roughly equal numbers of low and high frequency items. We further balanced 
items in the different groups for selection strength ratio, a measure obtained from Cameron Jones (2008) and defined as the frequency of the most common response divided by the second most common response; a high response strength ratio corresponds to a low selection demand and vice versa. We used these selection strength ratios in order to balance the selection demands for the high and low conditions across alternative test versions. In confirmation of this, an independent samples t-test revealed no significant difference in the high $(t(45)=-0.553, p=.583)$ and low $t(45)=-0.36, p=.871)$ conditions between the two versions of the task (see Table 3.5). There was also no significant difference in frequency ratings (high: $t(45)=1.202, p=.236$; low: $t(45)=-0.143, p=.887)$.

Table 3.5

The average response strength ratio and frequency values for the two versions of the verb generation task

Selection Strength Demand

\begin{tabular}{ccccc} 
Version & High & Low & High & Low \\
\hline 1 & 1.73 & 16.42 & 1828.48 & 172.14 \\
2 & 1.73 & 16.63 & 1611.86 & 177.91 \\
\hline
\end{tabular}

Frequency (CELEX LEMMA )
2.2 Procedure. In this task, participants view a pictured item, and had to produce the name of an action associated with that item (e.g., scissors -> “cut"). They were instructed to say "what the object does, or what is done with the object". They were then given the example of "dog", to which a person might respond "bark" or "fetch".

For each version of the task, a practice item was presented first. Feedback was given if the participant made an incorrect response on this item. This was to ensure participants were familiar with the task; this item was not included in further analyses. The experimental 
items were then presented in a fixed, pseudo-randomised order. As shown in Figure 3.6, each picture was presented alongside its written name. The target name was also presented aurally, simultaneously with the visual stimulus. This was done to reduce the likelihood that the participant would name the object, and it also acted as a marker to calculate response latencies. Presentation was self-paced; once the participant had made a response, the experimenter initiated the presentation of the word: 'Ready?' with a key press. This appeared for $100 \mathrm{~ms}$ and then the next experimental item appeared. If the participant made three incorrect responses, in which a noun was substituted instead of a verb (e.g., "moon"-> "night"), feedback was given from the experimenter ("remember in this task you need to name an action associated with the picture you see”). Feedback was only given once throughout the task.

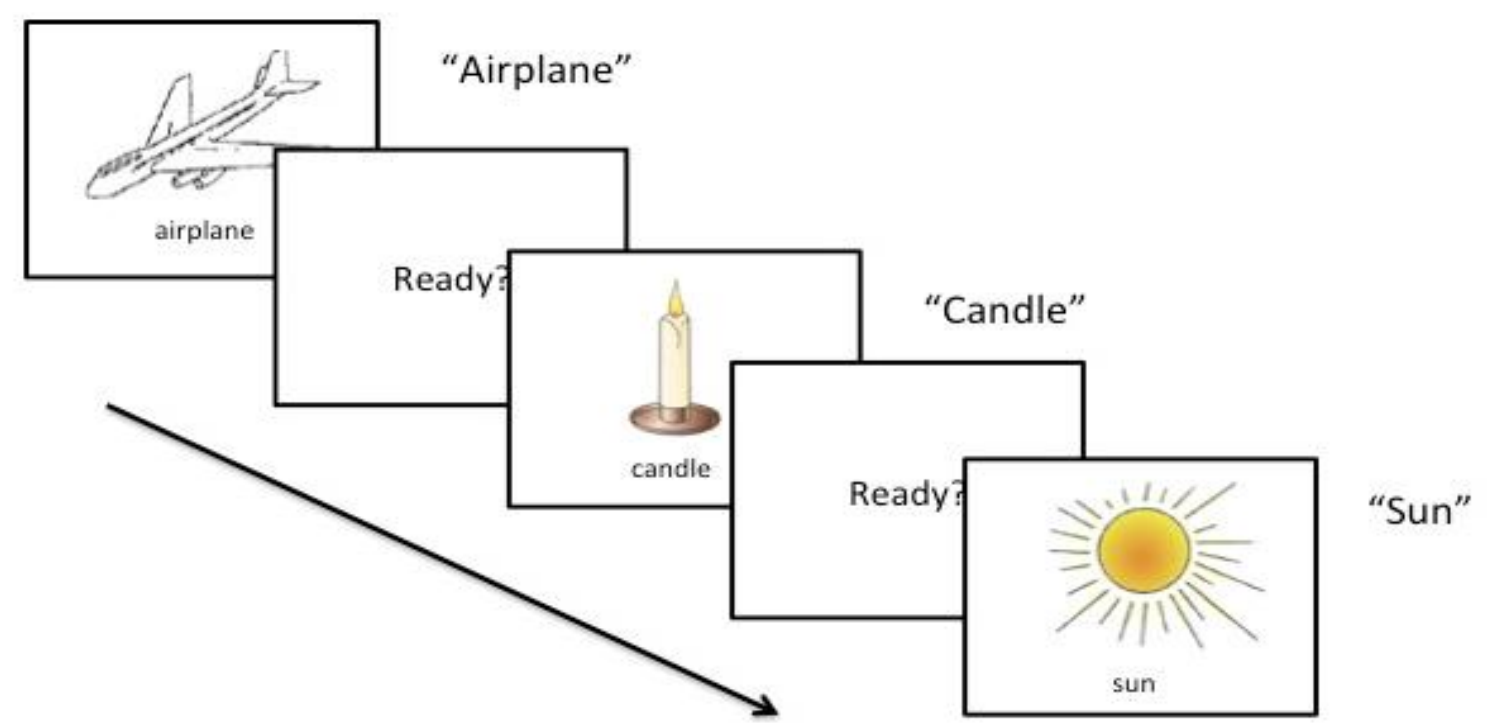

Figure 3.6. Item presentation in the verb generation task

\subsection{Response Scoring.}

2.3.1 Accuracy A response was considered correct if it consisted of a verb or action name that was: a) appropriate to the noun; and b) specific to the noun (e.g., ladder, "climb", 
is accepted, but ladder "use" was not). Inflectional forms of the verbs were also scored as correct (e.g., "climb" -> "climbed," or "climbing"). The total percentage of correct responses was calculated, as well as for both the selection and frequency manipulations.

2.3.2 Response Latencies. The procedure used here was the same as that described in Section 1.3.2, except in this task latencies were measured from the onset of the auditory noun, which accompanied the picture.

\section{Picture-Word Verification}

In this task, participants must determine if an aurally presented word matches a visually displayed object.

3.1 Materials. The 24 items used for this test were derived from a previously designed picture-word verification task, which comprised 50 target words (Wilshire, Keall, Stuart \& O'Donnell, 2007). These items were selected because they belonged to one of four semantic categories: animals, food, household objects, and weapons. The pictures used to depict these words were coloured line drawings and photographs adapted from the Rossion and Pourtois (2004) coloured Snodgrass-like drawings, and other public domain sources. All pictures yielded name agreement of $80 \%$ or more when piloted on a group of 70 participants of varying ages (see Wilshire, Keall, Stuart \& O’Donnell, 2007).

Each picture was presented four times, each time accompanied by a different auditory word: an identical word (which matched the picture's name); a phonologically related word, which shared at least the first two phonemes with the target and had the same number of syllables and stress pattern (e.g., chair-cheque); a semantically related word, which was from the same semantic category as the target word, as narrowly defined as possible (for example, horse was matched with deer, rather than simply another animal); or an unrelated word, which bore no semantic relationship to the target and shared no phonemes in the same 
position (e.g., grapes-puzzle). Frequency and length in syllables were balanced across the four different conditions (see Table 3.6).

The picture-word verification task was divided into two versions each consisting of 12 items. Frequency, semantic category and syllable length were balanced across the two versions (see Table 3.6). An independent samples t-test revealed no significant difference between syllable length and frequency between the two versions. When each picture was paired with each of its respective auditory words, this yielded a total of 48 trials per version. Participants were presented all four conditions involving each picture in the same session. Table 3.6

The average frequency (log CELEX frequency) and syllable length for the two versions of the picture-word verification task

Version 1

Version 2

\begin{tabular}{ccccc}
$\begin{array}{c}\text { Distractor } \\
\text { Type }\end{array}$ & Frequency & $\begin{array}{c}\text { Syllable } \\
\text { Length }\end{array}$ & Frequency & $\begin{array}{c}\text { Syllable } \\
\text { Length }\end{array}$ \\
\hline Identical & 2.86 & 1.75 & 2.87 & 1.75 \\
Phonological & 2.54 & 1.58 & 2.49 & 1.58 \\
Semantic & 2.52 & 1.42 & 2.54 & 1.5 \\
& & 1.67 & & 1.58 \\
Unrelated & 2.52 & & 2.54 & \\
\hline
\end{tabular}

3.2 Procedure. In this task, participants were simultaneously presented with a picture and an auditory word, and they had to judge if the auditory word matched the picture's name. This task was, therefore, a forced-choice task, where participants could only respond with a "yes" or "no". 
Each version of the task commenced with two unrelated practice items, which were not included in further analyses. If a participant made an incorrect response on any of these items, feedback was given by the experimenter. The experimental items were then presented, in fixed pseudo-random order. At the beginning of each trial, a fixation cross appeared. Then $100 \mathrm{~ms}$ later, the picture appeared, accompanied by a tone (see Figure 3.7). Presentation was self-paced; once the participant made a response the researcher initiated the presentation of the fixation cross with a key press.

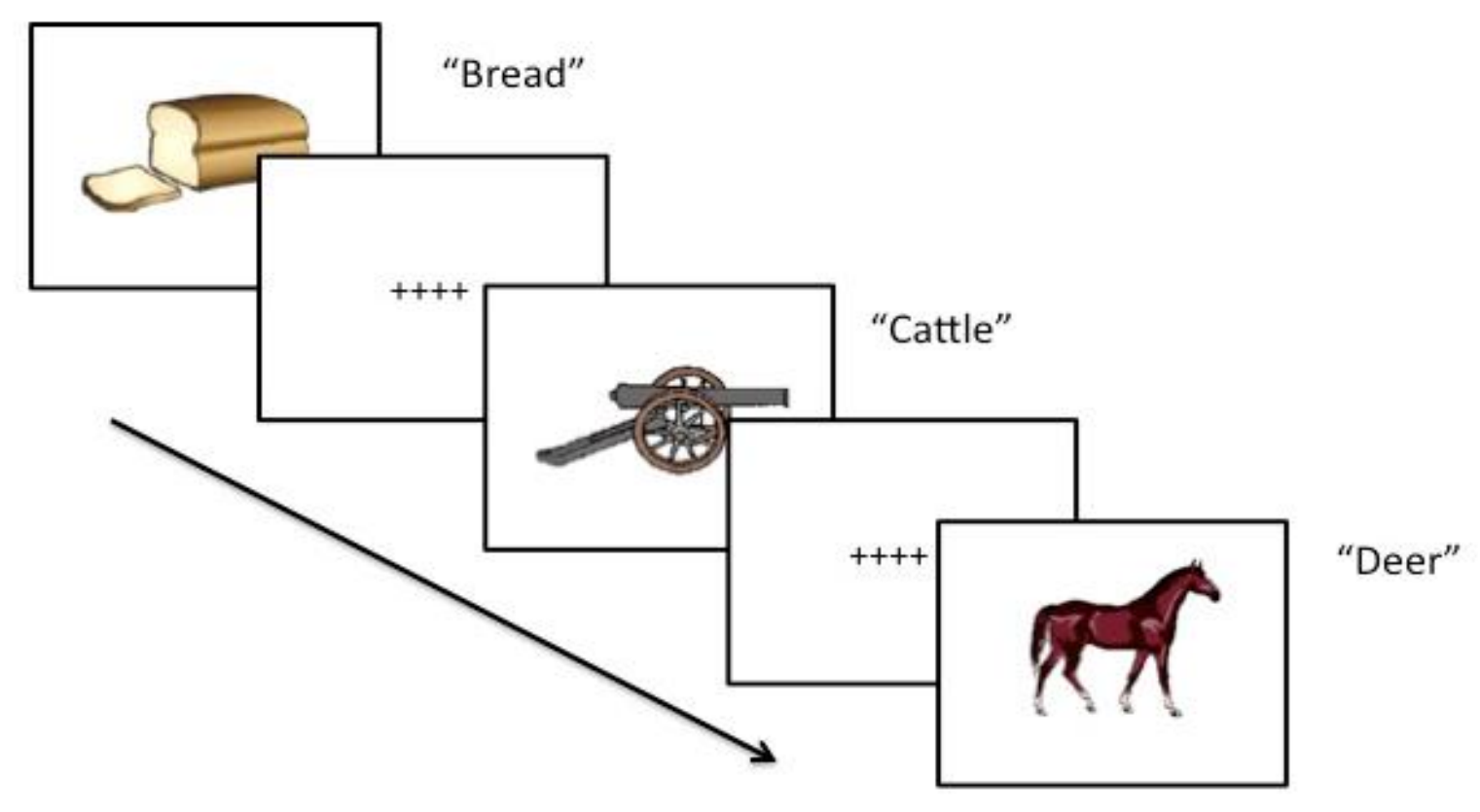

Figure 3.7. Item presentation in the picture-word verification task

3.3 Response Scoring. Responses were scored as correct or incorrect. In addition, response latencies were measured from the onset of the distractor to the onset of the participants' yes-no response, according to the procedures described in section 1.3.2. 


\section{Single Word Repetition}

In this task, participants were required to repeat an aurally presented word. The task consisted of two parts; the first consisted of real words that varied in their frequency and imageability ratings. The second consisted of a set of nonwords.

4.1 Materials. The 90 items used in this test consisted of 60 real words and 30 nonwords drawn from the PALPA word and nonword repetition test (PALPA Test 9: Kay et al, 1996). The same items were administered for both versions of the BLAST. The 60 real words comprised 30 high imageability and 30 low imageability words, and each of these sets was further divided into 15 high frequency and 15 low frequency words. The 30 nonwords were also drawn from the same PALPA subtest, each of which differs from one of the real words in the test by at least one letter (For example analogy -> atalogy).

4.2 Procedure. For this test, the experimenter pronounced a single word and participants were required to repeat that word immediately. For this task, the experimenter was oriented in a way that prevented the participant seeing their lips, in order to prevent lip reading. The experimental items were presented in two blocks - real and nonwords - and each block was presented in pseudo-random order.

4.3 Response Scoring. Each word response produced by the participant was scored as correct or incorrect. Latencies to initiate each response - measured from stimulus word onset to response onset - were also obtained, using the methods outlined in Section 1.3.2.

\section{Word and Nonword Reading.}

In this task, participants are required to read out loud a single word. This task consists of two parts; the first comprises of a set of real words that varied in word length and regularity. The second part comprises a set of nonwords.

5.1 Materials. The word reading section of the test comprised 28 real word items, the first 12 of which were taken from the PALPA Letter Length Spelling Test (PALPA Test 39; 
Kay et al., 1996). The first three items of this set had three letters, the next all had four letters, then five, and the final three items all had six letters. These blocks of words were presented in such a way that, as the test progressed, word length also increased. All items included in this section had an imageability rating score over six in the MRC psycholingustic database (Coltheart, 1981). This means that when normal (usually undergraduate) participants were asked to rate how easily they could visualise an image of the meaning of the word - on a scale from 1 to 7 - they rated these words at or above 6 (e.g., highly imageable). In addition, the words of differing length were matched for frequency, based on the logged frequency values from Kucera (1967) database (see Table 3.7); there were no reliable frequency difference amongst words from the four different length groups $(F(3,8)=.076, p=.971)$.

Table 3.7

Mean log frequency (Kucera, 1967) and imageability for the four blocks in the first section of the reading test

\begin{tabular}{cccc}
\hline Block & Syllable Length & Mean Frequency & $\begin{array}{c}\text { Mean } \\
\text { Imageability }\end{array}$ \\
\hline 1 & 1 & 1.83 & 6.38 \\
2 & 2 & 1.85 & 6.17 \\
3 & 3 & 1.86 & 6.24 \\
4 & 4 & 1.89 & 6.21 \\
\hline
\end{tabular}

The next 16 real word items in the single word reading task were taken from the Reading Word Subtest of the Comprehensive Aphasia Test (Swinburn, Porter \& Howard, 2005). These 16 items consisted of eight high frequency words (with a mean Kucera (1967) frequency of 260) and eight low frequency words (with a mean Kucera (1967) frequency of 
5.0). An independent samples t-test confirmed a statistically significant difference between the frequency conditions $(t(14)=4.57, p<.001)$. In each of these frequency groups, four of the items were high imageability words and four were low imageability words (high imageability words had a mean imageability rating of 6.04 , and low imageability words had a mean imageability rating of $3.73^{4}$ ). An independent samples t-test confirmed a statistically significant difference between the imageability conditions $(t(14)=15.559, p<.001)$. Further, sound-spelling regularity was balanced across these item groups (there were two regular words and two irregular words in each group). All words in this section were two syllables. Each word target item was presented on the centre of a laptop computer, in black size 60 font.

The nonword section of the task comprised 12 items taken from the PALPA Nonword Reading Task (Test 36: Kay et al., 1996). Similar to the first 12 words in the real word reading task, the first three items all had three letters (e.g., ked), the next all had four letters (e.g., shid), then five (e.g., glope) and the final three items all had six letters (e.g., churse).

5.2 Procedure. The word and nonword stimuli were presented in the centre of a computer screen, and participants were instructed to read the word aloud as quickly as possible. The task began with a practice item, which was not included in the response analysis. Prior to each item, a line of fixation crosses appeared on the screen for $100 \mathrm{~ms}$. Each word was presented simultaneously with a tone, to facilitate the subsequent recording of response latencies. The first 12 items in the reading test were presented in order of increasing length, as described in the previous section. The remaining 16 items were then presented in pseudo-random order. Once the participant made a response, the researcher initiated the presentation of the fixation cross with a key press.

In the nonword reading part of the task, the procedure was identical except that the participant was told the items he/she would see would be nonwords. The nonwords were

\footnotetext{
${ }^{4}$ Imageability ratings were derived from the MRC psycholingustic database (Coltheart, 1981)
} 
presented in order according of increasing length, commencing with the three-letter nonwords, and concluding with the six-letter nonwords.

5.3 Response Scoring. Each word response produced by the participant was scored as correct or incorrect. Latencies to initiate each response - measured from stimulus word onset to response onset - were also obtained, using the methods outlined in Section 1.3.2.

\section{Stroop}

The premise of this task is based on the classical Stroop task formulated by Stroop (1935) and others (for review see, Macleod, 1991). Specifically, participants are required to ignore the identity of a word, and name the colour the word is presented in.

6.1 Materials. The stimulus items consisted of 20 single colour name words selected randomly from a pool of eight different alternatives: pink, black, red, blue, green, orange, yellow and purple. There were two different conditions: congruent and incongruent (Stroop, 1935). The congruent condition consisted of six items and in this condition, the colour of the word matched that of the written word name (e.g., BLUE). The incongruent condition consisted of 13 items and in this condition, the colour of the word did not match the written word name (e.g., GREEN). Each word target item was presented on the centre of a laptop computer, in size 60 font.

6.2 Procedure. In this task, participants were required to ignore the identity of the word, and simply name the colour it was presented in. Instructions were presented on the computer screen and read verbatim by the experimenter. These were:

"In this task, you will see some words written in different colours. Your job is to name the colour that the word is written in. Ignore what the word actually says. All we want is the name of its colour. So for example, if you saw this word purple, you would answer "blue." Do you have any questions?" 
Two practice items were then presented to ensure familiarity with the task. Both items were examples from the incongruent condition. If participants got these items wrong, the experimenter gave feedback. The experimental items were then presented in a fixed pseudorandom order. Before each item, a fixation cross appeared for 100ms (see Figure 3.8). Then the word was presented simultaneously with a tone. The tone acted as a marker, to facilitate measurement of response latency. Once the participant had made a response, the researcher initiated the presentation of the next fixation cross with a key press.

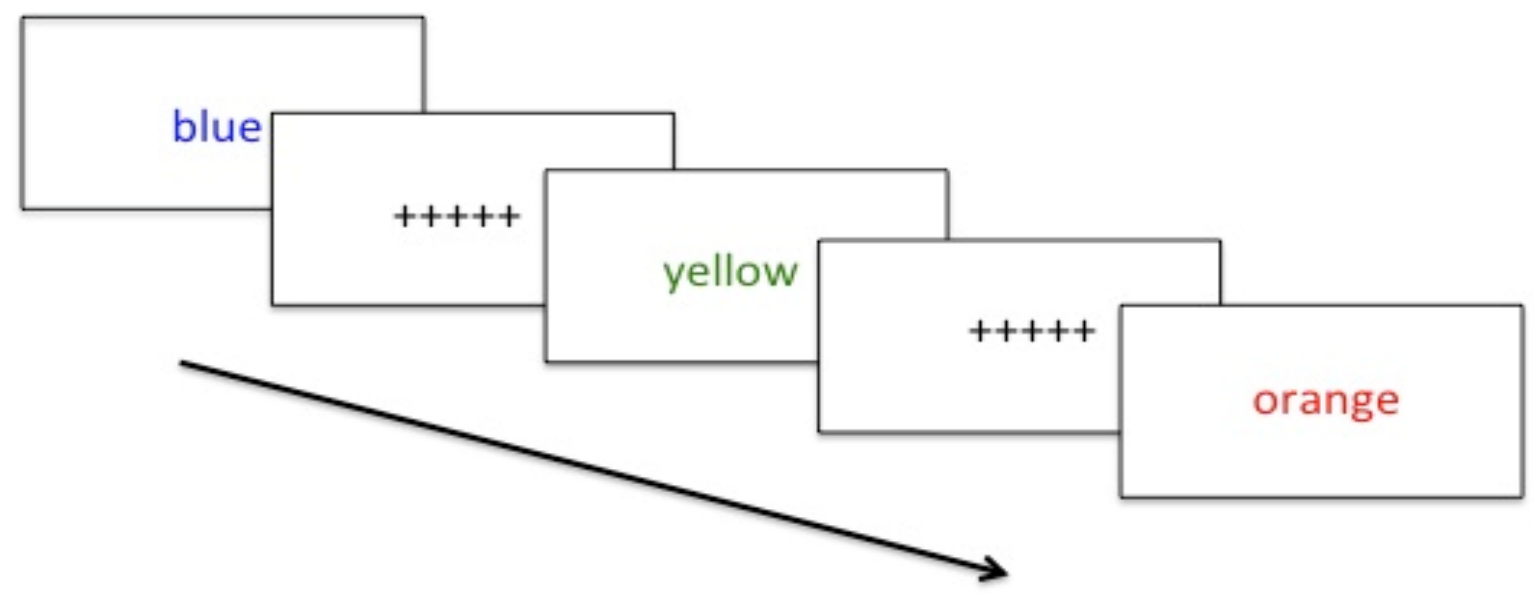

Figure 3.8. Item presentation in the Stroop task

6.3 Response Scoring. Each word response produced by the participant was scored as correct or incorrect. Latencies to initiate each response - measured from stimulus word onset to response onset - were also obtained, using the methods outlined in Section 1.3.2.

\section{Letter Fluency}

The letter fluency task is based on the oral Verbal Fluency task first developed by Arthur Benton more than 40 years ago (Mitrushina, Boone \& D'Elia, 1998). In this test, participants are provided orally with a letter of the alphabet and are required to say as many words as they can that begin with that letter within 60 seconds. 
7.1. Materials and Procedure. This test was based on standard administration and consisted of three phases, each phase involving of a different letter. The first letter was $F$, followed by $A$, and then $S$ (Spreen, 1998). The experimenter used a stopwatch to record 60 seconds, the time allocated for each phase.

The instructions given to participants were derived from Controlled Oral Word Association Test (Spreen, 1998). They were as follows:

"I will say a letter of the alphabet. Then I want you to give me as many words as you can that begin with that letter as quickly as you can. For instance, if I say 'B', you might give me 'bad', battle', 'bed'... I do not want to you to use words that are proper names such as 'Boston' or 'Bob'. Also, please do not use the same word again with a different ending such as 'eat' and 'eating'. Any questions? Begin when I say a letter. The first letter is 'F'. Go ahead."

After these instructions were given, the experimenter measured 60 seconds using a stopwatch. Once this time had elapsed, the experimenter instructed the participant as follows: "Your time is up. You next letter is A (S). Go ahead."

7.2 Response Scoring. All responses made by the participant that began with the allocated letter were scored as correct, with the exception of proper names and repetitions of or variations of the same word were excluded (e.g., fish, fishes, fishing). The total number of correct responses was calculated for each letter. Response latencies were not recorded for this task.

\section{Category Fluency}

The category fluency task was also based on standard administration (Spreen 1998), but consisted of two phases, each involving a different semantic category. The first category was animals, and the second was fruit. 
8.1 Materials and Procedure. The procedure for administration was as for the Letter Fluency task (Section 7), except for the instructions, which were as follows:

"This time I am going to say a category. Then I want you to give me as many words as that belong to that category, as quickly as you can. For instance If say countries, you might give me Australia, New Zealand etc. Any questions? Begin when I say a category. The first category is 'animals'. Go ahead."

After these instructions were given, the experimenter measured 60 seconds using a stopwatch. Once this time had elapsed the experimenter instructed the participant as follows: "Your time is up. Your next category is fruit. Go ahead."

8.2 Response Scoring. Responses were scored in the same way as for the Letter Fluency task (Section 7).

\section{Articulatory Agility Test}

This task is based on the Verbal Agility Subtest of the BDAE (Goodglass, Kaplan \& Barresi, 2001). In this task, participants are required to repeat a given word as many times as they can in a five-second period.

9.1 Materials and Procedure. The words used were the original seven items from the BDAE (Goodglass, Kaplan \& Barresi, 2001) subtest: mamma, tip-top, fifty-fifty, thanks, huckleberry, baseball player, and caterpillar. The experimenter used a stopwatch to record five seconds, the time allocated for each item. Instructions were as follows:

"I will say a word and you are required to continually repeat the following word as rapidly as you can within five seconds. Once this time is up, I will say another word and your job is to repeat that word just like you did before. Any questions?"

After these instructions were given, the experimenter measured five seconds using a stopwatch. Once this time had elapsed the experimenter concluded the trial and presented the next word item. 
9.2 Response Scoring. The total number of words correctly repeated for each item, within the designated time period was recorded. Response latencies were not recorded for this task. 
Table 3.8

Key variables manipulated in each of the tests within the protocol, and, where relevant, mean values for these variables.

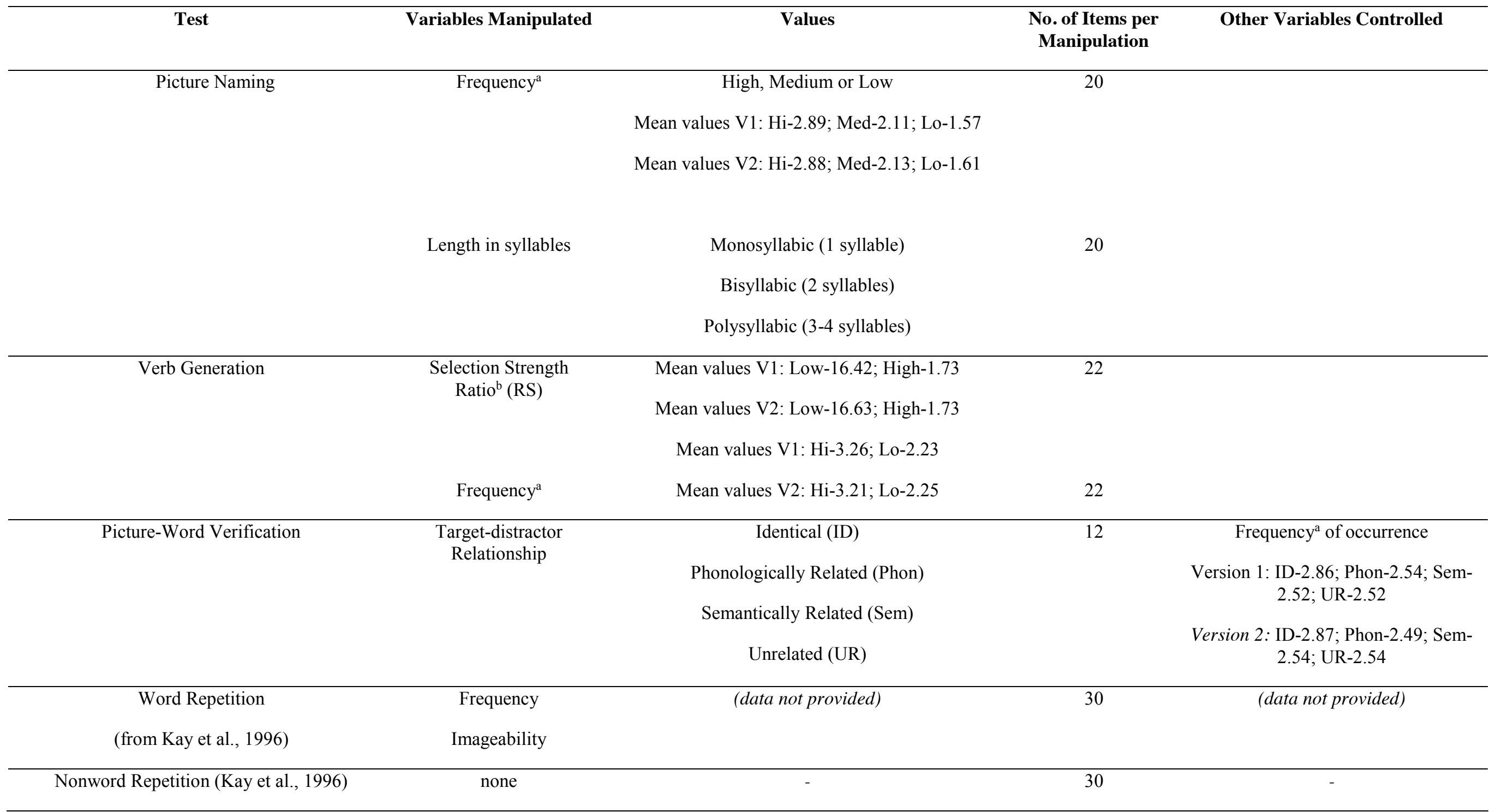




\begin{tabular}{|c|c|c|c|c|}
\hline Test & Variables Manipulated & Values & $\begin{array}{l}\text { No. of Items per } \\
\text { Manipulation }\end{array}$ & Other Variables Controlled \\
\hline \multirow[t]{2}{*}{ Stroop } & Congruency & Incongruent & 7 & \\
\hline & & Congruent & 13 & \\
\hline \multirow[t]{2}{*}{ Fluency } & Letter & $\mathrm{F}, \mathrm{A}, \mathrm{S}$ & & \\
\hline & Category & Animals, Fruit & & \\
\hline Articulatory Agility & Words & & 7 & \\
\hline \multirow[t]{3}{*}{ Reading } & Syllable Length & 1, 2, 3, 4 Syllables & 3 & $\begin{array}{l}\text { Frequency of occurrence: Mono- } \\
\text { 1.83; Bi-1.85; Tri-1.86; Quad-1.89 }\end{array}$ \\
\hline & Regularity & Regular & 8 & $\begin{array}{c}\text { Frequency }^{\mathrm{c}} \text { of occurrence: Regular- } \\
2.12 ; \text { Irrgular- } 2.12\end{array}$ \\
\hline & & Irregular & & \\
\hline Nonword Reading & none & & 12 & \\
\hline
\end{tabular}

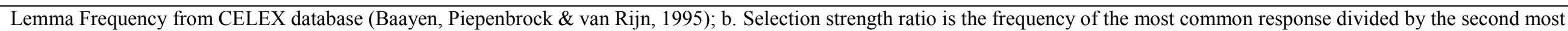
common response (Cameron Jones, 2008) c. Kucera (1967) frequency database from the English Lexicon Project (Balota et al., 2007) 


\section{General Procedure}

\section{Healthy Controls}

Testing occurred in one session and took approximately 45 minutes to complete. A Macintosh laptop was then set up directly in front of the participant and an audio tape recorder was then started to record the testing session. Task administration occurred in the following order: Picture naming Version one and two, Verb generation Version one and two, Picture-word verification Version one and two, real word and nonword repetition, real word and nonword reading, Stroop, letter and category fluency, and articulatory agility. PsyScope software (Cohen, MacWhinney, Flatt \& Provost, 1993) was used to visually present the computerised tasks on the Macintosh computer (picture naming, verb generation, picture word verification, reading and Stroop). The remaining two tasks - word/nonword repetition and articulatory agility - were administered directly by the experimenter. A stopwatch was used for the fluency and articulation tasks. A break was offered to each participant after completion of Version two of the picture-word verification. After completion of the test battery, participants were given a debrief form and thanked for their time.

\section{Brain Tumour Patients}

Preoperative Testing. Preoperative testing occurred once the patient had been admitted to the neurosurgical ward of Wellington Hospital. In most cases, this occurred the day before surgery, but in three cases, preoperative testing occurred the morning before surgery. On the surgical ward, the experimenter introduced himself to the patient and their family if applicable, and explained the purpose of the study. Before consent was obtained, the experimenter went through the information sheet in detail with the patient (see Appendix D), and their family and answered any questions they may have had. The participant was reminded that involvement in the study was voluntary, and if they declined to participate that this would not affect their subsequent treatment and care. Once informed consent was 
obtained, participants were asked to comment about any visual difficulties they might currently be experiencing. If a patient reported severe visual difficulties (for example, blind in one eye), preoperative testing was abandoned and they were thanked for their time. The experimenter noted on a patient's consent form, if they reported minor visual disturbances (for example: "I am having difficulty making out some objects"). During subsequent testing if these patients were notably struggling to elicit a response during a task with a strong visual component (e.g., picture naming), the experimenter asked the patient to comment if this was due to difficulties seeing the item on the screen. If this was confirmed by the patient, subsequent testing was abandoned and the patient was thanked for their time. Finally, each patient was also asked to comment on any speech or language difficulties they had experienced within the last three months. If the patient reported such an experience, this was noted on their consent form.

Patients were seated in front of a Macintosh laptop computer and an audiotape recorder was then started to record the testing session. Before testing was commenced patients were told that during testing if they experienced any discomfort or fatigue at any point to let the experimenter know straight away. Version one of the BLAST was then administered. Task administration occurred in the following order: picture naming, verb generation, picture-word verification, real word and nonword repetition, real word and nonword reading, Stroop, letter and category fluency, and articulatory agility. Two breaks were offered to each patient throughout the testing session. The first one was after completion of verb generation and the second after the completion of the Stroop task. All other aspects of the administration method were as for the healthy controls, with the following two exceptions. First, if the experimenter noticed any discomfort from the patient during the testing session, testing was immediately stopped, and a break was initiated. If the patient did not feel comfortable to continue testing, the rest of the session was abandoned. Second, during testing, 
if a medical staff member came to see the patient, testing was stopped and recommenced when the patient was available. Once preoperative testing was completed, the experimenter reminded the patient that they would be visited two days after their surgery for postoperative testing. Any questions the patient or their family had in regards to performance on the battery were also answered by the experimenter.

Postoperative testing. Postoperative testing occurred two-three days after surgery. This timing was variable due to individual differences in fatigue and postoperative discomfort, which constrained patient's ability to engage in testing. If a patient reported that they felt able to participate in postoperative testing, administration of the battery occurred in exactly the same manner as preoperative testing (outlined above). However, during postoperative testing, Version two of the BLAST was administered and there were no initial screening questions. In addition, if the patient reported discomfort or fatigue, or if the experimenter became aware of this, testing was immediately ceased, and resumed the following day. Once postoperative testing was completed, the experimenter answered any questions the patient or family member may have had in regards to test performance. 


\section{Chapter 4: Overall Task Performance}

Our first objective was to analyse patients' overall performance on the BLAST irrespective of the within-task manipulations. In order to achieve this, each patient's overall score on each task was compared with the average overall accuracy of the appropriate age matched control group using Crawford, Howell and Garthwaite's (1998) modified t-test. Impairment was defined as a significant difference in accuracy between the patient and control group, with $p<.05$. In addition, Chi-Square analysis was used to determine if a significant difference existed between a patient's preoperative and postoperative overall accuracy. Each patient's overall preoperative and postoperative score on each of the tasks within the BLAST is shown in Table 4.1.

Preoperatively, $94.3 \%$ of patients scored significantly below their respective control group in at least one task contained within the BLAST. In addition, as shown in Figure 4.1, preoperatively, these patients were most commonly impaired on one-three tasks within the BLAST. Similarly, postoperatively, $90.3 \%$ of patients scored significantly below their controls in at least one task within the BLAST, and these patients were also most commonly impaired on one-three tasks. 
Table 4.1

Tumour patients' overall scores on each task within the BLAST as a percentage both pre-and postoperatively

\begin{tabular}{|c|c|c|c|c|c|c|c|c|c|c|c|c|c|c|c|c|c|c|c|c|c|c|c|}
\hline & & \multicolumn{2}{|c|}{ Picture Naming } & \multicolumn{2}{|c|}{$\begin{array}{c}\text { Verb } \\
\text { Generation }\end{array}$} & \multicolumn{2}{|c|}{$\begin{array}{l}\text { Picture Word } \\
\text { Verification }\end{array}$} & \multicolumn{2}{|c|}{$\begin{array}{c}\text { Repetition } \\
\text { (Real) }\end{array}$} & \multicolumn{2}{|c|}{$\begin{array}{c}\text { Repetition } \\
\text { (Nonwords) }\end{array}$} & \multicolumn{2}{|c|}{$\begin{array}{c}\text { Reading } \\
\text { (Real) }\end{array}$} & \multicolumn{2}{|c|}{$\begin{array}{c}\text { Reading } \\
\text { (Nonwords) }\end{array}$} & \multicolumn{2}{|c|}{ Stroop } & \multicolumn{2}{|c|}{ Letter Fluency } & \multicolumn{2}{|c|}{$\begin{array}{l}\text { Category } \\
\text { Fluency }\end{array}$} & \multicolumn{2}{|c|}{ Articulation } \\
\hline & & $V I$ & $V 2$ & $V 1$ & $V 2$ & $V 1$ & $v 2$ & $v 1$ & $V 2$ & $v 1$ & $v 2$ & $V I$ & $V 2$ & $V 1$ & V2 & $V 1$ & $V 2$ & $V I$ & $V 2$ & $V I$ & $V 2$ & $V 1$ & $V 2$ \\
\hline \multicolumn{2}{|c|}{ Control Data (51+) } & 94 & 94 & 93 & 91 & 98 & 98 & 97 & 97 & 84 & 84 & 100 & 100 & 95 & 95 & 95 & 95 & 47 & 47 & 40 & 40 & 53 & 53 \\
\hline Name & $\begin{array}{c}\text { Tumour } \\
\text { Location }\end{array}$ & Pre & Post & Pre & Post & Pre & Post & Pre & Post & Pre & Post & Pre & Post & Pre & Post & Pre & $\begin{array}{c}\text { Pos } \\
t\end{array}$ & Pre & Post & Pre & Post & Pre & Post \\
\hline SM & L Frontal & 92 & 87 & $62 *$ & $78^{*}$ & na & na & 98 & 100 & na & na & na & na & na & na & 100 & 100 & 38 & 32 & 25 & 21 & na & na \\
\hline VD & L Frontal & 92 & $75^{*}$ & $60^{*}$ & $60^{*}$ & 98 & 100 & 100 & 100 & na & na & na & na & na & na & 90 & 100 & $6^{* *}$ & $15^{*}$ & $6^{*}$ & $9^{*}$ & 52 & 65 \\
\hline $\mathrm{RF}$ & L Frontal & 95 & 95 & $67 *$ & 80 & $92 *$ & 100 & 97 & 100 & 95 & 90 & 100 & 100 & $75^{*}$ & 83 & 100 & 100 & 30 & 31 & 15 & 27 & 46 & 44 \\
\hline SO & L Frontal & 82 & $82 *$ & $67^{*}$ & $64 *$ & $94 *$ & 98 & 100 & 100 & 100 & 90 & 100 & 100 & 100 & 92 & 95 & 80 & 24 & 40 & 25 & 30 & 28 & $25^{*}$ \\
\hline EMH & L Frontal & 88 & na & $71^{*}$ & na & 96 & na & 97 & na & 95 & na & na & na & na & na & 90 & na & $10^{*}$ & na & 23 & na & 53 & na \\
\hline EA & L Frontal & $63^{*}$ & $63 *$ & $78^{*}$ & $69^{*}$ & $94 *$ & $94 *$ & 97 & 100 & 85 & 100 & 100 & 100 & $67 *$ & $25^{*}$ & $75^{*}$ & 65 & $15^{*}$ & $14 *$ & 25 & 23 & 37 & 37 \\
\hline $\mathrm{JM}$ & L Frontal & 85 & $68^{*}$ & 82 & $67 *$ & 96 & 98 & 100 & 100 & 95 & 100 & 100 & 100 & 100 & 100 & 85 & 80 & $16^{*}$ & $8 * *$ & 21 & 18 & 29 & 31 \\
\hline $\mathrm{CR}$ & L Frontal & 88.33 & 95 & 93 & 82 & 100 & 94 & 97 & 100 & $60^{* *}$ & $70^{* *}$ & 100 & na & 92 & na & 85 & na & 37 & na & 25 & na & 38 & 0 \\
\hline $\mathrm{BCA}$ & L Posterior & $40^{*}$ & $42 *$ & $63^{*}$ & $77^{*}$ & $90^{*}$ & $73^{*}$ & 100 & 100 & na & na & na & na & na & na & na & na & 22 & 23 & $7^{*}$ & $8^{*}$ & 45 & 57 \\
\hline $\mathrm{BD}$ & L Posterior & 87 & $82^{*}$ & 84 & 93 & 98 & 100 & 100 & 100 & 85 & 100 & 100 & 100 & 100 & 100 & 95 & 90 & 34 & 25 & 24 & 25 & 54 & 61 \\
\hline LA & R Frontal & 92 & $80^{*}$ & 93 & 80 & $92 *$ & 98 & 99 & 99 & na & na & na & na & na & na & 100 & 100 & 24 & 19 & 23 & 18 & 49 & 46 \\
\hline $\mathrm{CM}$ & R Frontal & 95 & 88 & $73 *$ & $71^{*}$ & 96 & $96^{*}$ & 98 & 99 & na & na & na & na & na & na & 100 & 100 & $13 *$ & 30 & na & 30 & 73 & 75 \\
\hline LW & R Frontal & 93 & 97 & $76^{*}$ & $62 *$ & $94 *$ & $92 *$ & 100 & 100 & 85 & 90 & 100 & 100 & 100 & 100 & 95 & 90 & 20 & 20 & 34 & 33 & 51 & 54 \\
\hline ES & R Frontal & $72^{*}$ & $70^{*}$ & 78 & 78 & $61^{*}$ & na & $82 *$ & 97 & na & na & na & na & na & na & na & na & $8 * *$ & $7 * *$ & 17 & 19 & na & na \\
\hline RG & R Posterior & $70^{*}$ & $56^{*}$ & 84 & $60^{*}$ & $90^{*}$ & $96^{*}$ & 94 & 96 & na & na & na & na & na & na & $75^{*}$ & 100 & 21 & 24 & 25 & 25 & 37 & 36 \\
\hline TKH & R Posterior & $65^{*}$ & $67 *$ & $78^{*}$ & 80 & $88^{*}$ & 98 & 93 & 97 & na & na & na & na & na & na & 85 & 80 & 18 & 20 & 20 & 22 & 45 & 57 \\
\hline JAS & R Posterior & 92 & na & $51^{*}$ & na & 98 & na & 97 & na & na & na & $78.57^{*}$ & na & na & na & na & na & $10^{*}$ & na & $13 *$ & na & na & na \\
\hline AEK & R Posterior & 85 & $78^{*}$ & 87 & $69^{*}$ & 98 & 100 & 98 & 95 & 85 & 95 & 100 & 100 & 100 & 92 & 100 & 100 & 33 & 32 & 35 & 37 & 54 & 35 \\
\hline PAJ & $\begin{array}{l}\text { Multiple } \\
\text { Lesions }\end{array}$ & 87 & $83 *$ & $64 *$ & $67^{*}$ & 96 & $90^{*}$ & 97 & $72 *$ & 85 & na & 96.43 & 100 & na & $75^{*}$ & na & na & $3^{* *}$ & $12^{*}$ & $7^{*}$ & $9^{*}$ & 51 & 32 \\
\hline $\mathrm{SMC}$ & R Frontal & 88 & 87 & 93 & 84 & 98 & 100 & 100 & 100 & 95 & 100 & 100 & 100 & 100 & 100 & $75^{*}$ & 90 & 20 & 27 & 29 & 28 & 50 & 47 \\
\hline MRO & R Frontal & 95 & 97 & 87 & 80 & $90^{*}$ & $86^{*}$ & 100 & 100 & 100 & 100 & 100 & 100 & 100 & na & 95 & 100 & 41 & 57 & 28 & 41 & na & na \\
\hline $\mathrm{RJ}$ & L Posterior & 92 & na & 80 & na & $96^{*}$ & na & 100 & na & 90 & na & 100 & na & $58^{*}$ & na & 85 & na & $18^{*}$ & na & 23 & na & 49 & na \\
\hline GP & R Frontal & $73 *$ & $77 *$ & 87 & $76^{*}$ & $92 *$ & 98 & 93 & 100 & 90 & 95 & 100 & 100 & 100 & 100 & $70^{*}$ & $70 *$ & 22 & 33 & 25 & 32 & 40 & 49 \\
\hline
\end{tabular}




\begin{tabular}{|c|c|c|c|c|c|c|c|c|c|c|c|c|c|c|c|c|c|c|c|c|c|c|c|}
\hline \multirow[b]{2}{*}{ MR } & \multirow[b]{2}{*}{ L Frontal } & \multicolumn{2}{|c|}{ Picture Naming } & \multicolumn{2}{|c|}{$\begin{array}{c}\text { Verb } \\
\text { Generation }\end{array}$} & \multicolumn{2}{|c|}{$\begin{array}{l}\text { Picture Word } \\
\text { Verification }\end{array}$} & \multicolumn{2}{|c|}{$\begin{array}{c}\text { Repetition } \\
\text { (Real) }\end{array}$} & \multicolumn{2}{|c|}{$\begin{array}{l}\text { Repetition } \\
\text { (Nonwords) }\end{array}$} & \multicolumn{2}{|c|}{$\begin{array}{c}\text { Reading } \\
\text { (Real) }\end{array}$} & \multicolumn{2}{|c|}{$\begin{array}{c}\text { Reading } \\
\text { (Nonwords) }\end{array}$} & \multicolumn{2}{|c|}{ Stroop } & \multicolumn{2}{|c|}{ Letter Fluency } & \multicolumn{2}{|c|}{$\begin{array}{l}\text { Category } \\
\text { Fluency }\end{array}$} & \multicolumn{2}{|c|}{ Articulation } \\
\hline & & 83 & na & 80 & na & 96 & na & 100 & na & 95 & $\mathrm{Na}$ & 100 & na & 100 & na & $50^{*}$ & na & $10^{*}$ & na & 18 & na & 39 & na \\
\hline \multicolumn{2}{|c|}{ 30-50 Patient Group } & 96 & 94 & 94 & 91 & 98 & 100 & 100 & 100 & 98 & 98 & 100 & 100 & 93 & 93 & 99 & 99 & 46 & 46 & 42 & 42 & 60 & 60 \\
\hline $\mathrm{PM}$ & L Frontal & 98 & $87 *$ & $82 *$ & $62 *$ & na & na & 98 & 100 & na & na & na & na & na & na & $90^{*}$ & $45^{*}$ & 35 & $1^{* *}$ & 32 & $11^{*}$ & 70 & 65 \\
\hline $\mathrm{DA}$ & L Frontal & $90^{*}$ & $78^{*}$ & $80^{*}$ & 87 & 96 & 96 & 100 & 99 & na & na & na & na & na & na & $95^{*}$ & 100 & 38 & $23^{*}$ & 35 & $13^{*}$ & 41 & 40 \\
\hline $\mathrm{EH}$ & L Frontal & 100 & $88 *$ & 93 & 87 & $83^{*}$ & 100 & 100 & 100 & na & na & na & na & na & na & 100 & 100 & 30 & 34 & $11^{*}$ & 27 & $37^{*}$ & 58 \\
\hline $\mathrm{LC}$ & L Frontal & 97 & $77 *$ & 91 & $67 *$ & 96 & $92 *$ & 100 & 100 & 95 & 100 & na & 100 & na & 100 & 100 & 100 & 34 & $14 *$ & 41 & $13 *$ & 46 & 46 \\
\hline $\mathrm{CA}$ & L Frontal & $70 *$ & $82 *$ & $55^{*}$ & $69 *$ & $77^{*}$ & 100 & 100 & 100 & 95 & 100 & 100 & 100 & 100 & 100 & 100 & 100 & $15^{*}$ & 27 & $8^{*}$ & 18 & 43 & $36 *$ \\
\hline CG & L Frontal & 95 & 92 & $82 *$ & $78^{*}$ & 100 & 100 & 100 & 100 & 100 & 95 & 100 & 100 & na & na & 100 & 100 & 38 & 42 & 24 & 40 & 57 & 57 \\
\hline AVG & L Posterior & 98 & 98 & 93 & 87 & 96 & 98 & 100 & 100 & na & na & na & na & na & na & 100 & 100 & 36 & 29 & 21 & 31 & 77 & 78 \\
\hline $\mathrm{AM}$ & L Posterior & $80^{*}$ & $77 *$ & $78^{*}$ & 87 & 98 & na & 100 & 99 & na & na & na & na & na & na & 95 & 100 & $18^{*}$ & $11^{*}$ & 17 & $13^{*}$ & 50 & 63 \\
\hline $\mathrm{JB}$ & R Posterior & 93 & 97 & $73 *$ & $69 *$ & 98 & 100 & 100 & 100 & na & na & na & na & na & na & 100 & 100 & 44 & 43 & 41 & 42 & 74 & 71 \\
\hline $\mathrm{AE}$ & $\begin{array}{l}\text { Multiple } \\
\text { Lesions }\end{array}$ & $83 *$ & $70^{*}$ & $73^{*}$ & $56^{*}$ & 98 & $94 *$ & 98 & 100 & 95 & 100 & $92.86^{*}$ & 100 & $50^{*}$ & $33^{*}$ & $85^{*}$ & $80 *$ & 29 & 29 & 33 & 18 & 47 & 53 \\
\hline TT & R Frontal & 92 & $87^{*}$ & $82 *$ & $73 *$ & 96 & $94 *$ & 100 & 100 & 95 & 100 & 100 & 100 & 91.67 & 100 & $95^{*}$ & $90^{*}$ & 32 & 36 & 34 & 42 & 56 & 57 \\
\hline $\mathrm{KB}$ & R Frontal & $88^{*}$ & na & 87 & na & 98 & na & 100 & na & 95 & na & 100 & na & 100 & na & 100 & na & 25 & na & 27 & na & 46 & na \\
\hline DAP & L Frontal & 95 & na & $69^{*}$ & na & 98 & na & 100 & na & 100 & na & 100 & na & 100 & na & $95^{*}$ & na & 44 & na & 39 & na & 52 & na \\
\hline $\mathrm{TF}$ & L Frontal & $87 *$ & $82 * *$ & $60^{*}$ & $58^{*}$ & 96 & 100 & $92 *$ & $88^{*}$ & $80^{*}$ & $60^{*}$ & 100 & 100 & 100 & 92 & $75^{*}$ & $75^{*}$ & $6^{*}$ & $8^{*}$ & $10^{*}$ & 16 & $22 *$ & $31^{*}$ \\
\hline $\mathrm{TD}$ & L Frontal & 95 & na & 89 & na & 96 & na & 100 & $\mathrm{Na}$ & 90 & na & 100 & na & $92 *$ & na & 100 & na & 19 & na & 27 & na & na & na \\
\hline DF & R Frontal & 93 & 93 & $84 *$ & $84 *$ & $94 *$ & 100 & 100 & 98 & 100 & 95 & 100 & 100 & $83 *$ & $92 *$ & 100 & 100 & 17 & 18 & 30 & 24 & 43 & 45 \\
\hline
\end{tabular}




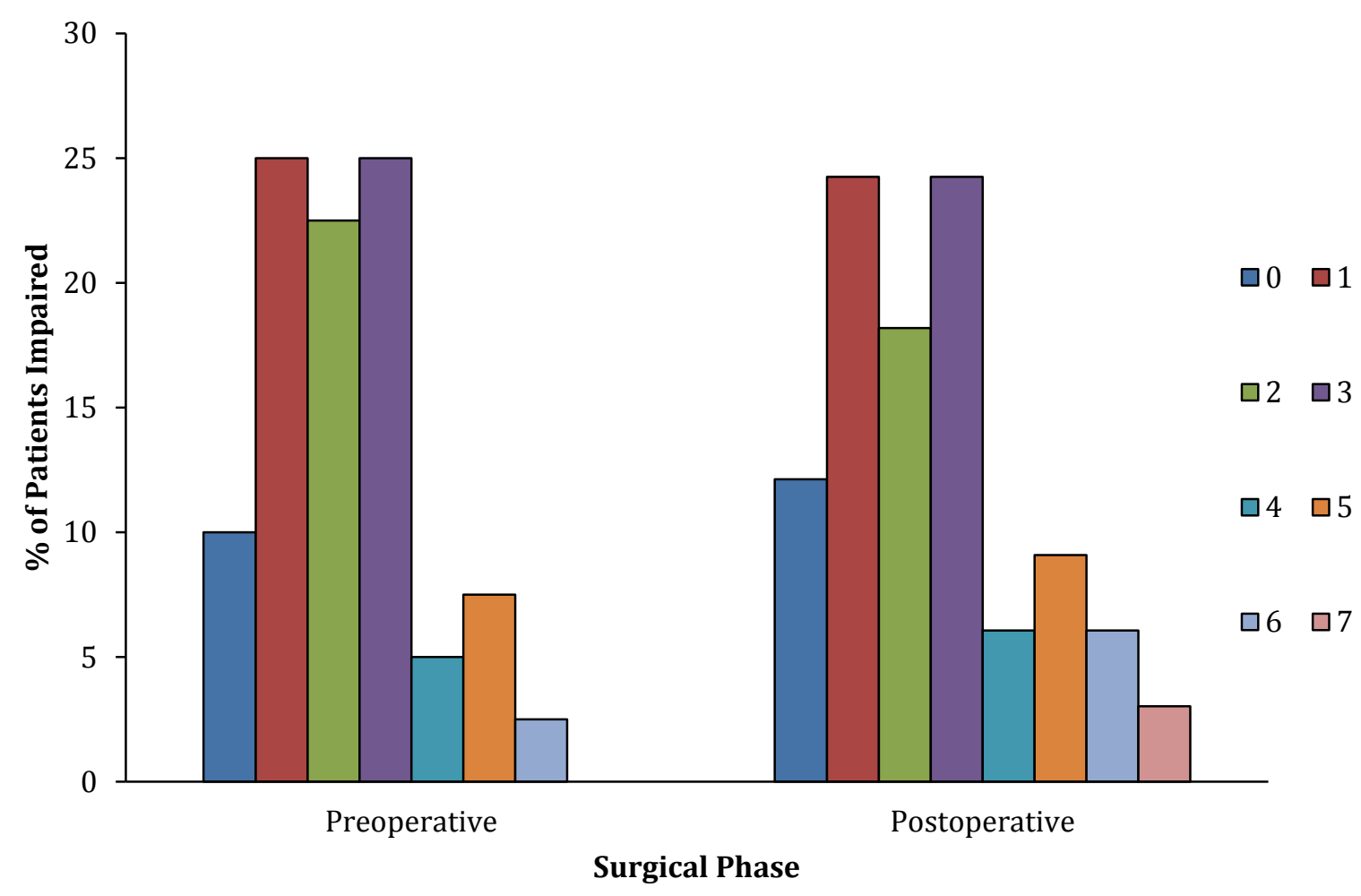

Figure 4.1. The proportion of patients who were significantly different to their appropriate control group based on the number of tasks within BLAST that were completed both pre and postoperatively

\section{Sensitivity of Specific Tasks}

Figure 4.2 shows the percentage of patients who scored significantly below their controls on each of the BLAST subtasks. Preoperatively, verb generation was the most sensitive task. Specifically, $60 \%$ of patients were impaired on this task. Other tasks that were particularly sensitive preoperatively were picture naming $(32.5 \%)$, picture-word verification (37.5\%), Stroop (30.6\%), letter fluency (37.5\%) and category fluency $(22.5 \%)$. Real word and nonword repetition, as well as real word reading and articulation were the least sensitive tasks within BLAST preoperatively, with less than $10 \%$ of patients impaired.

Patients' postoperative performance in each of the tasks within BLAST were very similar to their preoperative performance, with two exceptions: 1) there was a significantly higher incidence of impairment in picture naming postoperatively when compared to preoperatively $(66.67 \%)\left(\chi^{2}(1)=8.59, p=.003\right)$, and 2$)$ there was a trend towards fewer 
impairments on the Stroop postoperatively (16.67\%); however, this difference was not significant $\left(\chi^{2}(1)=1.72, p=.190\right)$.

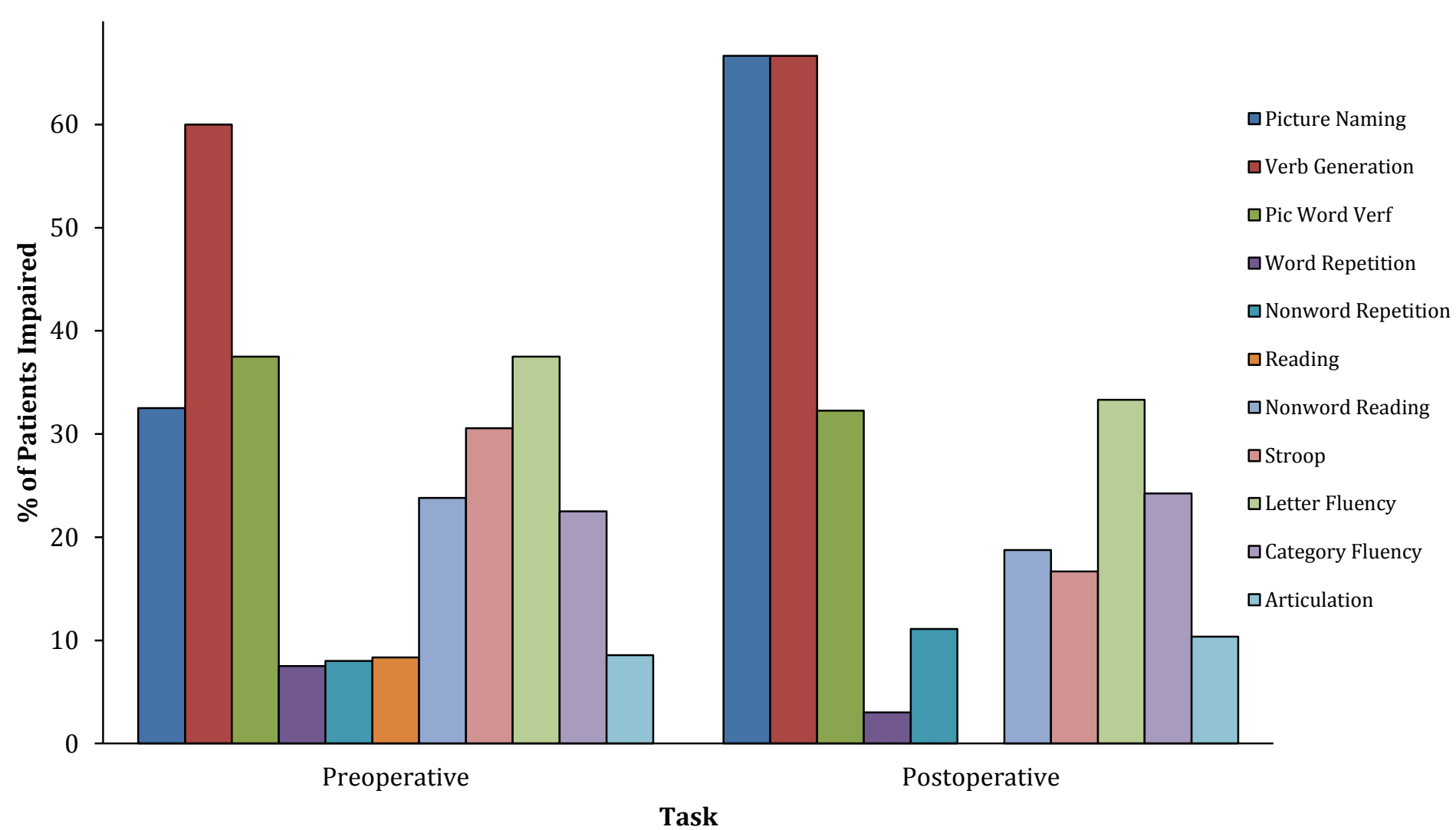

Figure 4.2. The proportion of patients significantly different to their appropriate control group for each task within BLAST both pre- and postoperatively

\section{Effects of Tumour Localisation and Tumour Characteristics}

For those tasks on which more than $25 \%$ of patients were impaired, we further broke down scores into four groups based on broad lesion localisation: left frontal and posterior, and right frontal and posterior (categorisation into these four anatomical groups is outlined in Chapter 3). Tasks meeting this criterion were: picture naming, verb generation, picture-word verification, Stroop, letter fluency and category fluency. Figure 4.3 shows the relevant results. We used logistic regression to investigate if there were any significant differences in the incidence of impairment across these different anatomical groups. 
Preoperatively, the only task that showed reliable anatomical localisation was category fluency. Specifically, patients with a left posterior tumour were significantly more impaired on this task than all other anatomical groups $\left(\chi^{2}(3)=5.92, p=.0150\right)$. In addition, postoperatively, patients with a left hemisphere lesion performed significantly more poorly in this task than patient with a right hemisphere lesion $\left(\chi^{2}(1)=9.56, p=.0020\right)$, but there was no reliable posterior/anterior difference at this testing phase. In addition, postoperatively, the incidence of letter fluency deficits also differed significantly across the four anatomical groups; patients with a left frontal lesion were significantly more impaired on this task than all than all other anatomical groups $\left(\chi^{2}(3)=18.92, p=.0259\right)$. There was no other task postoperatively that showed anatomical specificity. 


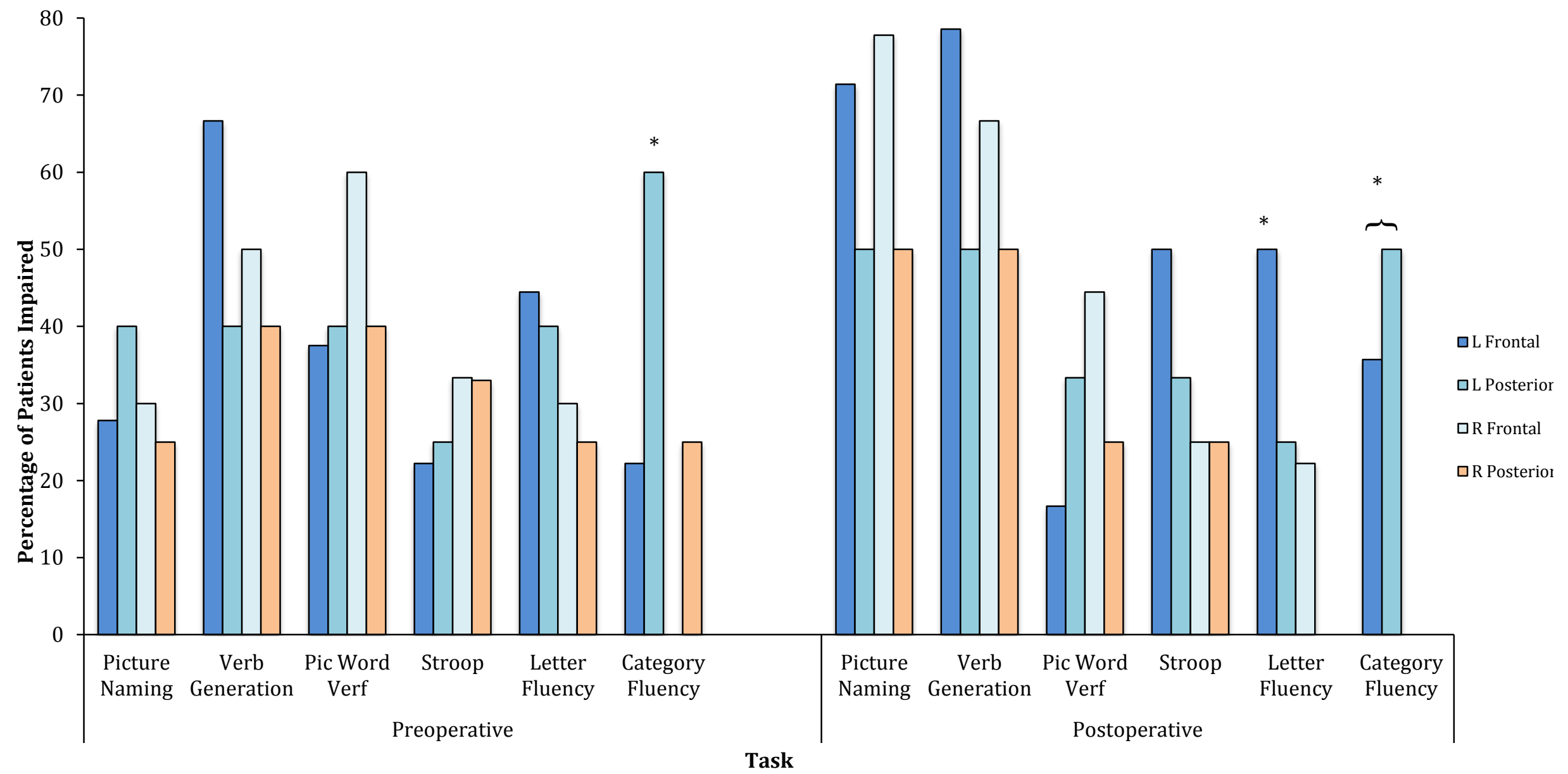

Figure 4.3. The percentage of patients impaired in the most sensitive tasks within BLAST by tumour localisation both pre- and postoperatively 
Based on their histological reports, patients were also categorised into three groups: low-grade malignancy (pre=10; post=7); high-grade malignancy $($ pre=14; post=11), and meningioma (pre $=12 ;$ post $=11)^{5}$. As shown in Table 4.2, there was no significant difference in impairment between patients with a high-grade malignant tumour and the other two tumour types. However, there was a trend towards patients with a high grade malignant tumour performing more poorly in picture naming in both pre and postoperatively. Patients with a low grade malignant tumour or a meningioma did not perform significantly more poorly on these tasks.

Table 4.2

The percentage of patients impaired on each task in the BLAST by tumour type both pre and postoperatively

\begin{tabular}{ccccccccc}
\hline Task & High & Low & Meningioma & $\begin{array}{c}\boldsymbol{p} \\
\text { value }\end{array}$ & High & Low & Meningioma & $\begin{array}{c}\boldsymbol{p} \\
\text { value }\end{array}$ \\
\hline $\begin{array}{c}\text { Picture } \\
\text { Naming }\end{array}$ & 50 & 0 & 42 & .062 & 91 & 57 & 55 & .056 \\
$\begin{array}{c}\text { Verb } \\
\text { Generation }\end{array}$ & 57 & 70 & 42 & .940 & 75 & 67 & 45 & .300 \\
$\begin{array}{c}\text { Picture- } \\
\text { Word }\end{array}$ & 57 & 22 & 50 & .920 & 18 & 29 & 36 & .433 \\
Verification & & & & & & & & \\
Stroop & 33 & 33 & 25 & .813 & 9 & 29 & 11 & .463 \\
$\begin{array}{c}\text { Letter } \\
\text { Fluency }\end{array}$ & 50 & 50 & 8 & .193 & 36 & 25 & 40 & .841 \\
$\begin{array}{c}\text { Category } \\
\text { Fluency }\end{array}$ & 21 & 33 & 8 & .967 & 30 & 13 & 30 & .627 \\
\hline
\end{tabular}

${ }^{5}$ Two patients were excluded from this analysis due to the following: no histological report in medical records $(\mathrm{N}=1)$, and a cavernoma tumour $(\mathrm{N}=1)$ 
Patients were further categorised into two groups based on the presence of oedema surrounding the tumour (Oedema present, $\mathrm{N}=18$; Oedema absent, $\mathrm{N}=16)^{6}$.The percentage of patients impaired on each of the tasks within the BLAST based on this categorisation is presented in Table 4.3. A Chi-Square analysis revealed no significant difference between the oedema present and the no oedema present on any task within the BLAST either pre- or postoperatively.

Table 4.3

The percentage of patients impaired on each task in the BLAST by tumour oedema presence both pre and postoperatively

Preoperative

Postoperative

\begin{tabular}{ccccccc} 
Cognitive Skill & Yes & No & $\boldsymbol{p}$ value & Yes & No & $\boldsymbol{p}$ value \\
\hline Picture Naming & 28 & 38 & .591 & 69 & 63 & .271 \\
Verb Generation & 67 & 56 & .403 & 69 & 63 & .851 \\
Picture-Word Verification & 47 & 40 & .406 & 36 & 20 & .564 \\
Stroop & 33 & 19 & .744 & 46 & 29 & .643 \\
Letter Fluency & 50 & 31 & .436 & 33 & 31 & .739 \\
Category Fluency & 27 & 39 & .816 & 28 & 31 & .519 \\
\hline
\end{tabular}

As shown in Table 4.4, simple regression analysis revealed negative correlations between tumour volume and overall task performance on all tasks preoperatively. However, none of these correlations reached statistical significance. Due to the considerable changes that are likely to occur to tumour voume during surgery, this analysis was not computed postoperatively.

\footnotetext{
6 Four patients were excluded from this analysis. This was either because there was no pathological report in their medical records or oedema presence/absence was not specified in their medical records
} 
Table 4.4

Correlations coefficient calculated for the relationship between tumour volume and overall task performance both pre- and postoperatively

\begin{tabular}{ccc}
\hline Task & $\begin{array}{c}\text { Preoperative } \\
\text { Correlation } \\
\text { Coefficient }\end{array}$ & $\boldsymbol{p}$ value \\
\hline Picture Naming & -0.160 & .344 \\
Verb Generation & -0.042 & .804 \\
Picture-Word & -0.106 & .544 \\
Verification & & \\
Stroop & -0.246 & .217 \\
Letter Fluency & -0.32 & .851 \\
Category Fluency & -0.05 & .500 \\
\hline
\end{tabular}

\section{General Comments}

Our first step in exploring our participants' performance on the BLAST was to examine their overall performance on each subtask. We found that a staggering proportion of patients were significantly different to their respective control group in at least one task within the BLAST (preoperatively $=94 \%$; postoperatively $=90 \%$ ). Furthermore, patients' impairments were generally quite selective: the majority were impaired on one to three tasks, and this was true both pre and postoperatively. With regard to specific task sensitivity, verb generation was the most sensitive task. Other sensitive tasks were picture naming, pictureword verification, Stroop, letter fluency and category fluency. Real word and nonword repetition, as well as real word reading and articulation, were the least sensitive tasks. Finally, the only subtask where there was a significant change in the incidence of impairment across surgical phase was picture naming: the percentage of patients impaired on this task significantly increased from the preoperative to the postoperative phase. 
Our explorations of the effect of broad tumour localisation and tumour characteristics (pathology, oedema and volume) revealed few statistically reliable findings. At the broadest level the lack of differentiation in task performance between left hemisphere and right hemisphere tumours was surprising. This finding suggests that task performance may be highly susceptible to nonlingustic factors that could also impact on performance (this issue will be discussed in more detail in the general discussion). Although at a more specific level we did find evidence for an association of category fluency with the left hemisphere posterior tumours preoperatively, and letter fluency with left hemisphere anterior tumours postoperatively. Although the number of patients in some of our anatomical groups, particularly the posterior groups, was low so the ability to conduct these types of analysis is constrained by the limited power evident in our sample. The malignancy of the tumour had no significant effect on overall performance, although there was a trend towards patients with a high grade tumour performing more poorly in picture naming. Finally, we also found that tumour oedema and tumour volume had no significant effect on overall task performance on any task within the BLAST either pre- or postoperatively.

Our exploration of overall task performance has revealed that the picture naming task has relatively high sensitivity. Not only was this task able to detect a high incidence of impairments in our sample, but it was also the only task that found a significant difference in the incidence of impairment due to surgical phase and tumour malignancy. The picture naming task arguably recruits a range of different language skills that likely involve an extensive network of cortical structures involving the left frontal and posterior regions (see esp. Baldo, Arévalo, Patterson \& Dronkers, 2013; Damasio et al 1996, 2004; Hillis et al., 2006). As a result, the picture naming task may be more likely detect a language impairment adherent in our sample due to the range of language skills involved in the task. However, at the current point we are unable to determine what specific language skill or skills is driving 
this impairment. Our next step therefore is to specifically assess these language skills, by deriving a measure based on current cognitive and neuropsychological theories of language. 


\section{Chapter 5: Examination of Core Cognitive Skills}

In order to further investigate the language capabilities of surgical brain tumour patients, each patient's performance on the BLAST was further used to generate estimates of their ability with respect to the 11 core cognitive skills identified in Figure 2.1. To do this, we used patients' pattern of performance across the various tasks and manipulations to derive key performance measures. These measures were then combined in various ways in order to establish an overall quantified value for each core cognitive skill. Table 5.1 outlines each cognitive skill and the key performance measures that were contributed to its operationalisation.

Each key performance measure listed in Table 5.1 was first obtained for each participant. It was then converted to a $\mathrm{Z}$ score using the mean and SD of the relevant control group, using the following formula:

$$
\mathrm{Z}=\frac{(\text { Individual Score }- \text { Control Group Mean })}{\text { Control Group Standard Deviation }}
$$

Key performance measures (see Table 5.1), expressed as Z scores were then combined in various ways in order to derive a total score for each of the core cognitive skills. Table 5.2 outlines the specific equations used to calculate each of these skills. Finally, each patient's cognitive skill expressed as a Z score using the measures for the relevant control group, was converted to a $\mathrm{T}$ score for ease of interpretation using the following formula:

$$
\mathrm{T} \text { Score }=(Z \text { Score } \mathrm{x} 10)+50
$$

For previous studies that have used $\mathrm{T}$ scores to present aggregate neuropsychological measures, see Miller and Rohling (2001). 
Table 5.1

Summary of the task profiles associated with each of the 11 core cognitive skills assessed by the BLAST

\begin{tabular}{|c|c|c|c|}
\hline Cognitive Skill & BLAST Profile & Key Performance Measures & Abbreviation \\
\hline \multirow[t]{3}{*}{$\begin{array}{l}\text { Accessing Semantic } \\
\text { Knowledge }\end{array}$} & $\begin{array}{l}\text { 1. Semantic confusions in picture word } \\
\text { verification }\end{array}$ & $\begin{array}{l}\text { 1. Semantic confusion accuracy minus } \\
\text { phonological confusion accuracy in picture- } \\
\text { word verification }\end{array}$ & 1. PicWrdSem \\
\hline & $\begin{array}{l}\text { 2. Verbal fluency: poorer category than letter } \\
\text { fluency }\end{array}$ & $\begin{array}{l}\text { 2. Average category fluency score minus average } \\
\text { letter fluency score }\end{array}$ & 2. CatFlu \\
\hline & $\begin{array}{l}\text { 3. Production of semantic errors in picture } \\
\text { naming }\end{array}$ & $\begin{array}{l}\text { 3. Total semantic errors } \\
\text { in picture naming }\end{array}$ & 3. PicNamSemEr \\
\hline \multirow[t]{4}{*}{ Lexical Selection } & 4. Strong frequency effect in picture naming & $\begin{array}{c}\text { 4. Accuracy in low frequency items in picture } \\
\text { naming compared to medium and high frequency } \\
\text { items }\end{array}$ & 4. PicNamFreq \\
\hline & $\begin{array}{l}\text { 5. Production of omission errors in picture } \\
\text { naming }\end{array}$ & 5. Total omission errors in picture naming & 5. PicNamOmisEr \\
\hline & $\begin{array}{l}\text { 6. Production of semantic errors in picture } \\
\text { naming }\end{array}$ & 6. Total semantic errors in picture naming & 6. PicNamSemEr \\
\hline & 7. Normal picture word verification & 7. Total accuracy in picture word verification & 7. PicWrd \\
\hline \multirow[t]{4}{*}{$\begin{array}{l}\text { Phonological } \\
\text { Encoding }\end{array}$} & 8. Strong length effects in picture naming & $\begin{array}{l}\text { 8. Accuracy in polysyllabic items in picture naming } \\
\text { compared to bisyllabic and monosyllabic items }\end{array}$ & 8. PicNamLeng \\
\hline & 9. Abnormal latency in single word repetition & 9. Average latency in real word repetition & 9.ReptLat \\
\hline & $\begin{array}{l}\text { 10. Abnormal latency in single word } \\
\text { repetition }\end{array}$ & 10. Average latency in real word repetition & 10.NonReptLat \\
\hline & $\begin{array}{l}\text { 11. Production of phonological errors in picture } \\
\text { naming }\end{array}$ & 11. Total phonological errors in picture naming & 11.PicNamPhonErr \\
\hline
\end{tabular}




\begin{tabular}{|c|c|c|c|}
\hline Cognitive Skill & BLAST Profile & Key Performance Measures & Abbreviation \\
\hline & 12. Normal articulatory agility & 12. Total score in articulatory agility & 12. ArticAgil \\
\hline \multirow[t]{3}{*}{$\begin{array}{l}\text { Auditory Word } \\
\text { Recognition }\end{array}$} & $\begin{array}{l}\text { 13. Phonological confusions in picture word } \\
\text { verification }\end{array}$ & $\begin{array}{l}\text { 13. Phonological confusions accuracy minus } \\
\text { semantic confusion in picture word verification }\end{array}$ & 13. PicWrdPhon \\
\hline & 14. Abnormal latency in repetition & 14. Average latency in real word repetition & 14.ReptLat \\
\hline & $\begin{array}{l}\text { 15. Poor repetition accuracy relative to picture } \\
\text { naming accuracy }\end{array}$ & $\begin{array}{l}\text { 15. Repetition accuracy minus picture naming } \\
\text { accuracy }\end{array}$ & $\begin{array}{l}\text { 15. ReptAcc - } \\
\text { PicNam }\end{array}$ \\
\hline \multirow[t]{5}{*}{$\begin{array}{l}\text { Goal-Driven Response } \\
\text { Selection }\end{array}$} & 16. Abnormal selection effect in verb generation & $\begin{array}{l}\text { 16. Accuracy in low selection items compared to } \\
\text { high selection items }\end{array}$ & 16. VerbSelection \\
\hline & 17. Abnormal congruency effect in Stroop & & \\
\hline & & $\begin{array}{l}\text { 17. Accuracy in incongruent items compared to } \\
\text { congruent items }\end{array}$ & 17. Stroop \\
\hline & 18. Poor letter fluency & & \\
\hline & & 18. Overall letter fluency score & 18.LettFlu \\
\hline Verb Retrieval & $\begin{array}{l}\text { 19. Poor verb generation in low selection items } \\
\text { relative to picture naming }\end{array}$ & $\begin{array}{l}\text { 19. Accuracy in verb generation high selection } \\
\text { minus accuracy in picture naming }\end{array}$ & 19. Verb - PicNam \\
\hline Verbal STM & 20. Poor nonword repetition & 20. Nonword repetition accuracy & 20. NonReptAcc \\
\hline $\begin{array}{l}\text { Visual Word } \\
\text { Recognition }\end{array}$ & 21. Strong length effect in single word readings & $\begin{array}{l}\text { 21. Response latency to read single words as word } \\
\text { length increases (length effect) }\end{array}$ & 21. ReadLengEff \\
\hline $\begin{array}{l}\text { Orthographic- } \\
\text { Phonological } \\
\text { Mapping }\end{array}$ & 22. Poor nonword readings & 22. Accuracy in nonword reading & 22. NonRead \\
\hline $\begin{array}{l}\text { Orthographic- } \\
\text { Semantic Mapping }\end{array}$ & $\begin{array}{l}\text { 23. Poor reading of irregular words relative to } \\
\text { regular words }\end{array}$ & $\begin{array}{l}\text { 23.Accuracy in irregular word reading relative to } \\
\text { regular word reading }\end{array}$ & $\begin{array}{l}\text { 23. IrrRead - } \\
\text { RegRead }\end{array}$ \\
\hline $\begin{array}{l}\text { Articulatory-Motor } \\
\text { Planning }\end{array}$ & 24. Poor articulatory agility score & 24. Total score in articulatory agility & 24. ArticAgil \\
\hline
\end{tabular}


Table 5.2

Formulae used to calculate each cognitive skill using the key performance measures outlined in Table 5.1. Notes that also calculations utilised the standardised Z-scores for each performance measure

\section{Cognitive Skill $\quad$ Formula used to Operationalise}

\begin{tabular}{|c|c|}
\hline Accessing Semantic Knowledge & $\begin{array}{c}=\text { Mean }(\text { PicWrdSem }+ \text { CatFlu }+ \\
\text { PicNamSemErr })\end{array}$ \\
\hline Lexical Selection & $\begin{array}{c}=\text { Mean }(\text { PicNamFreq }+ \text { PicNamOmisEr }+ \\
\text { PicNamSemErr })- \text { PicWrd }^{7}\end{array}$ \\
\hline Phonological Encoding & $\begin{array}{c}=\text { Mean (PicNamLeng }+ \text { ReptLat }+ \\
\text { NonRepLat }+ \text { PicNamPhonErr })- \\
\text { ArticAgil }\end{array}$ \\
\hline Auditory Word Recognition & $\begin{array}{c}=\text { Mean }(\text { PicWrdPhon }+ \text { ReptLat }+ \\
(\text { ReptAcc }- \text { PicNam }))\end{array}$ \\
\hline Goal-Driven Response Selection & $\begin{array}{c}=\text { Mean }(\text { VerbSelection }+ \text { Stroop } \\
+ \text { LettFlu })\end{array}$ \\
\hline Verb Retrieval & $=($ Verb - PicNam $)$ \\
\hline Verbal Short Term Memory & $=$ NonReptAcc \\
\hline
\end{tabular}

Verbal Short Term Memory

$=$ NonReptAcc

\footnotetext{
${ }^{7}$ For this measure we only wanted to consider scores where the patients frequency effect in picture naming (4) and their omission (5) and semantic errors (3) was larger than what would be expected based on their performance in picture-word verification. Therefore a patient's score for lexical selection was only calculated when the Mean score of (PicNamFreq +PicNamOmisEr+ PicNamSemErr) was lower than PicWrd. If a patient had a score of 6 that was larger than the mean score of $(4+5+3)$, these were scored as the respective control group's score for lexical selection.
} 
Cognitive Skill

Visual Word Recognition

Orthographic-Phonological Mapping

Orthographic-Semantic Mapping

Articulatory-Motor Planning
Formula used to Operationalise

$=$ ReadLengEff

$=$ NonRead

$=$ IrrRead - RegRead

$=$ ArticAgil 


\section{Overall Cognitive Skill Profile}

Each patient's score on the 11 core cognitive skills was compared with the mean core cognitive skill of their age matched control group using the Crawford, Howell, and Garthwaite (1998) modified t-test. Impairment was defined as a significant difference in performance between the patient's and control group's core skill scores, with $p<.05$. Individual patient's preoperative and positive core cognitive skill scores are presented in Table 5.3.

\section{Table 5.3}

The scores derived for each cognitive skill for each patient who completed the BLAST both pre and postoperatively

\begin{tabular}{|c|c|c|c|c|c|c|c|c|c|c|c|c|c|c|c|c|c|c|c|c|c|c|c|}
\hline & & \multicolumn{2}{|c|}{$\begin{array}{c}\text { Accessing } \\
\text { Semantic } \\
\text { Knowledge } \\
\end{array}$} & \multicolumn{2}{|c|}{ Lexical Selection } & \multicolumn{2}{|c|}{$\begin{array}{c}\text { Phonological } \\
\text { Encoding }\end{array}$} & \multicolumn{2}{|c|}{$\begin{array}{c}\text { Goal Driven } \\
\text { Response } \\
\text { Selection } \\
\end{array}$} & \multicolumn{2}{|c|}{ Verb Retrieval } & \multicolumn{2}{|c|}{$\begin{array}{c}\text { Auditory Word } \\
\text { Identification }\end{array}$} & \multicolumn{2}{|c|}{ Verbal STM } & \multicolumn{2}{|c|}{$\begin{array}{c}\text { Articulatory } \\
\text { Motor Planning }\end{array}$} & \multicolumn{2}{|c|}{$\begin{array}{c}\text { Visual Word } \\
\text { Identification } \\
\end{array}$} & \multicolumn{2}{|c|}{$\begin{array}{c}\begin{array}{c}\text { Orthographic- } \\
\text { Phonological } \\
\text { Mapping }\end{array} \\
\end{array}$} & \multicolumn{2}{|c|}{$\begin{array}{c}\text { Orthographic- } \\
\text { Semantic } \\
\text { Mapping } \\
\end{array}$} \\
\hline & & $V 1$ & $V 2$ & $V 1$ & $V 2$ & $V I$ & $V 2$ & VI & $V 2$ & $V 1$ & $v 2$ & $V 1$ & $V 2$ & $v 1$ & $V 2$ & $V 1$ & $V 2$ & $V 1$ & $V 2$ & $V 1$ & $V 2$ & $V 1$ & $v 2$ \\
\hline \multicolumn{2}{|c|}{ Controls (51+) } & 50.00 & 50.00 & 45.67 & 46.92 & 53.01 & 53.01 & 52.63 & 55.50 & 50.00 & 50.00 & 48.84 & 54.79 & 48.84 & 54.79 & 50.00 & 50.00 & 50.00 & 50.00 & 50.00 & 50.00 & 50.00 & 50.00 \\
\hline Name & Location & Pre & Post & Pre & Post & Pre & Post & Pre & Post & Pre & Post & Pre & Post & Pre & Post & Pre & Post & Pre & Post & Pre & Post & Pre & Post \\
\hline SM & L Frontal & 38.19 & 44.9 & 43.60 & 34.34 & 51.55 & 54.76 & 41.94 & 30.27 & $18.04^{*}$ & 50.13 & 53.83 & 62.18 & & & & & & & & & & \\
\hline VD & L Frontal & 47.44 & 38.79 & 38.83 & $17.74 *$ & 43.89 & 70.98 & $25.78^{*}$ & $9.62 *$ & $5.52^{*}$ & 48.19 & 55.52 & 63.89 & & & 49.58 & 51.10 & & & & & & \\
\hline $\mathrm{RF}$ & L Frontal & 45.11 & 53.2 & 45.67 & 46.92 & 68.73 & 71.46 & 39.74 & 40.25 & $18.76^{*}$ & 45.83 & 58.55 & 56.7 & 56.67 & 53.72 & 45.05 & 43.54 & 54.48 & 46.20 & $22.23 *$ & 34.13 & 52.36 & 52.36 \\
\hline SO & L Frontal & 30.60 & 37.12 & 45.67 & 46.92 & 64.52 & 68.33 & 44.85 & 33.29 & $31.13 *$ & 41.11 & 63.07 & 63.32 & 59.61 & 53.72 & $31.46 *$ & $29.19 *$ & 48.33 & 40.10 & 57.94 & 46.04 & 52.36 & 52.36 \\
\hline EMH & L Frontal & 52.11 & & 45.67 & & 43.04 & & 29.72 & & $28.15^{*}$ & & 52.41 & & 56.67 & & 45.05 & & & & & & & \\
\hline EA & L Frontal & 40.9 & 64.82 & 45.67 & 46.92 & 40.34 & 53.27 & 27.36 & $25.12^{*}$ & 43.86 & 57.38 & 60.68 & 63.06 & 50.78 & 59.61 & 38.26 & 38.26 & $14.96^{*}$ & 35.02 & $10.33^{*}$ & $-49.2^{*}$ & 52.36 & 9.93 \\
\hline $\mathrm{JM}$ & L Frontal & 39.4 & 34.46 & 41.41 & $20.29 *$ & 67.42 & 53.27 & 41.44 & 29.42 & 39.18 & 51.57 & 59.03 & 67.23 & 56.67 & 59.61 & $32.21^{*}$ & 33.72 & 32.96 & $20.00^{*}$ & 57.94 & 57.94 & 52.36 & 52.36 \\
\hline $\mathrm{CR}$ & L Frontal & 44.4 & 51.10 & 38.61 & 46.92 & 58.26 & 47.22 & 45.06 & & 57.17 & 40.79 & 50.98 & 50.68 & 36.05 & 41.94 & 39.01 & & 38.35 & & 46.04 & & & \\
\hline $\mathrm{BCA}$ & L Posterior & $-20.9^{*}$ & $-19.1 *$ & $-9.10^{*}$ & 46.92 & 65.36 & $45.60^{*}$ & 34.81 & 34.44 & 82.98 & 70.54 & 90.67 & 116.43 & & & 44.3 & 53.36 & & & & & & \\
\hline $\mathrm{BD}$ & L Posterior & 36.4 & 39.94 & 43.69 & $13.02 *$ & 51.30 & 42.83 & 42.62 & 44.06 & 46.99 & 66.36 & 60.98 & 62.71 & 50.78 & 59.61 & 51.1 & 56.38 & 46.26 & 53.00 & 57.94 & 57.94 & 52.36 & 52.36 \\
\hline
\end{tabular}




\begin{tabular}{|c|c|c|c|c|c|c|c|c|c|c|c|c|c|c|c|c|c|c|c|c|c|c|c|}
\hline \multirow[b]{2}{*}{ LA } & \multirow[b]{2}{*}{$\mathrm{R}$ Frontal } & \multicolumn{2}{|c|}{$\begin{array}{c}\text { Accessing } \\
\text { Semantic } \\
\text { Knowledge }\end{array}$} & \multicolumn{2}{|c|}{ Lexical Selection } & \multicolumn{2}{|c|}{$\begin{array}{l}\text { Phonological } \\
\text { Encoding }\end{array}$} & \multicolumn{2}{|c|}{$\begin{array}{c}\text { Goal Driven } \\
\text { Response } \\
\text { Selection } \\
\end{array}$} & \multicolumn{2}{|c|}{ Verb Retrieval } & \multicolumn{2}{|c|}{$\begin{array}{c}\text { Auditory Word } \\
\text { Identification }\end{array}$} & \multicolumn{2}{|c|}{ Verbal STM } & \multicolumn{2}{|c|}{$\begin{array}{c}\text { Articulatory } \\
\text { Motor Planning }\end{array}$} & \multicolumn{2}{|c|}{$\begin{array}{l}\text { Visual Word } \\
\text { Identification }\end{array}$} & \multicolumn{2}{|c|}{$\begin{array}{c}\text { Orthographic- } \\
\text { Phonological } \\
\text { Mapping } \\
\end{array}$} & \multicolumn{2}{|c|}{$\begin{array}{c}\text { Orthographic- } \\
\text { Semantic } \\
\text { Mapping } \\
\end{array}$} \\
\hline & & 43.6 & 50.00 & 45.67 & 39.92 & 61.79 & 60.33 & 45.36 & 33.83 & 52.46 & 57.87 & 57.84 & 57.91 & & & 47.32 & 45.05 & & & & & & \\
\hline $\mathrm{CM}$ & R Frontal & 46.75 & 57.59 & 45.68 & 46.92 & 42.71 & 43.47 & 32.98 & 44.63 & $18.76^{*}$ & 33.41 & 59.27 & 55.64 & & & 65.45 & 66.96 & & & & & & \\
\hline LW & R Frontal & 53.52 & 72.80 & 45.67 & 46.92 & 51.78 & 49.01 & 44.75 & 47.09 & $27.51^{*}$ & $8.55^{*}$ & 54.86 & 45.26 & 50.78 & 53.72 & 48.83 & 51.10 & 37.05 & 70.93 & 57.94 & 57.94 & 52.36 & 52.36 \\
\hline ES & R Frontal & 32.43 & 42.08 & 45.67 & 42.67 & 36.41 & 36.42 & 35.32 & $23.00^{*}$ & 59.41 & 69.83 & 54.39 & 62.17 & & & & & & & & & & \\
\hline RG & R Posterior & 42.08 & 45.61 & 45.67 & $27.62^{*}$ & 67.98 & 43.09 & 58.22 & 38.68 & 66.76 & 62.75 & 78.29 & 76.55 & & & 38.26 & 37.5 & & & & & & \\
\hline TKH & R Posterior & 35.31 & 38.87 & 45.67 & 40.08 & 46.24 & 37.17 & 39.31 & 38.68 & 73.82 & 78.73 & 74.17 & 64.22 & & & 44.3 & 53.36 & & & & & & \\
\hline JAS & R Posterior & 50.70 & & 43.95 & & 40.73 & & 46.79 & & $14.92 *$ & & 46.62 & & & & & & $-357^{*}$ & & & & -8.25 & \\
\hline AEK & R Posterior & 42.95 & 47.30 & 45.67 & 46.92 & 58.18 & 70.10 & 43.39 & 39.28 & 64.85 & 50.05 & 60.55 & 58.91 & 50.78 & 56.67 & 51.10 & 41.28 & 38.26 & 35.64 & 57.94 & 46.04 & 52.36 & 52.36 \\
\hline PAJ & $\begin{array}{l}\text { Multiple } \\
\text { Lesions }\end{array}$ & 48.00 & 60.7 & 45.67 & 46.92 & 57.07 & 73.02 & $11.91 *$ & 7.97* & 54.35 & 50.78 & 58.12 & $33.99 *$ & 50.78 & & 48.83 & 34.48 & 36.39 & $29.41^{*}$ & & & 9.93 & 52.36 \\
\hline SMC & R Frontal & 46.51 & 51.4 & 45.67 & $29.90^{*}$ & 61.26 & 55.9 & 49.35 & 44.7 & 66.56 & 50.46 & 60 & 58.7 & 56.67 & 59.61 & 48.07 & 45.81 & 47.94 & 40.80 & 57.94 & & 52.36 & 52.36 \\
\hline MRO & R Frontal & 35.67 & 46.58 & 45.67 & 46.92 & 56.41 & 53.52 & 46.26 & 43.77 & 44.37 & 38.85 & 60.89 & 70.19 & 59.61 & 59.61 & & & 45.52 & 40.75 & 57.94 & & 52.36 & 52.36 \\
\hline RJ & L Posterior & 50.60 & & 45.67 & & 58.03 & & 44.61 & & $29.85^{*}$ & & 51.77 & & 53.72 & & 47.32 & & 51.85 & & $1.59^{* *}$ & & 52.36 & \\
\hline GP & R Frontal & 49.42 & 37.40 & 45.67 & 46.92 & 45.7 & 47.19 & 39.18 & 38.76 & 68.48 & 46.93 & 60.95 & 59.51 & 53.72 & 56.67 & 40.52 & 47.32 & $-11.9^{*}$ & 56.46 & 57.94 & 57.94 & 52.36 & 52.36 \\
\hline MR & L Frontal & 46.82 & & 45.67 & & 59.56 & & 34.74 & & 45.86 & & 54.34 & & 56.67 & & 39.77 & & 35.34 & & 57.94 & & 52.36 & \\
\hline Cont & Is (30-50) & 50.5 & 52.46 & 47.85 & 46.57 & 50.04 & 50.99 & 49.10 & 50.9 & 50.00 & 50.00 & 49.59 & 49.61 & 50.00 & 50.00 & 50.00 & 50.00 & 50.00 & 50.00 & 50.00 & 50.00 & 50.00 & 50.00 \\
\hline PM & L Frontal & 46.8 & $29.4 *$ & 47.85 & $26.29 *$ & 51.54 & 35.29 & 51.5 & $-44.4 *$ & $28.58^{*}$ & $31.42^{*}$ & 46.56 & 52.31 & & & 60.66 & 55.36 & & & & & & \\
\hline DA & L Frontal & $30.7^{*}$ & 35.85 & 40.61 & 46.57 & 34.97 & 66.49 & 43.65 & $25.07 *$ & 34.61 & 68.74 & 65.27 & 70.42 & & & 29.94 & 28.88 & & & & & & \\
\hline $\mathrm{EH}$ & L Frontal & 38.82 & 38.53 & 47.85 & 40.04 & 83.86 & 57.64 & $15.44 *$ & $23.95 *$ & 37.2 & 56.8 & 56.42 & 60.24 & & & 25.7 & 47.94 & & & & & & \\
\hline $\mathrm{LC}$ & L Frontal & 46.00 & 49.00 & 47.85 & 46.57 & 65.37 & 65.25 & 42.4 & $28.12 *$ & 48.22 & 41.86 & 56.09 & 62.42 & & & 35.23 & 35.23 & & 64.26 & & 57.58 & & 52.36 \\
\hline $\mathrm{CA}$ & L Frontal & $24.86^{*}$ & 39.44 & 47.85 & 31.50 & $19.99 *$ & 39.84 & $-5.3^{*}$ & 43.79 & $23.67^{*}$ & $24.95 *$ & 79.85 & 46.28 & 41.23 & 55.56 & 32.06 & 24.64 & $8.26^{*}$ & $15.13 *$ & 57.58 & 57.58 & 52.36 & 52.36 \\
\hline CG & L Frontal & 50.82 & 49.10 & 38.87 & 46.57 & 42.14 & 56.2 & 39.16 & 44.54 & 36.72 & 34.07 & 45.78 & 52.96 & 41.23 & 55.56 & 46.89 & 46.89 & 52.91 & 50.35 & & & 52.36 & 52.36 \\
\hline AVG & L Posterior & 37.3 & 34.38 & 47.85 & 46.57 & 43.56 & $31.85^{*}$ & 46.25 & 40.29 & 42.36 & 36.27 & 54.83 & 49.55 & & & 68.07 & 69.13 & & & & & & \\
\hline
\end{tabular}




\begin{tabular}{|c|c|c|c|c|c|c|c|c|c|c|c|c|c|c|c|c|c|c|c|c|c|c|c|}
\hline \multirow[b]{2}{*}{$\mathrm{AM}$} & \multirow[b]{2}{*}{ L Posterior } & \multicolumn{2}{|c|}{$\begin{array}{c}\text { Accessing } \\
\text { Semantic } \\
\text { Knowledge } \\
\end{array}$} & \multicolumn{2}{|c|}{ Lexical Selection } & \multicolumn{2}{|c|}{$\begin{array}{l}\text { Phonological } \\
\text { Encoding }\end{array}$} & \multicolumn{2}{|c|}{$\begin{array}{l}\text { Goal Driven } \\
\text { Response } \\
\text { Selection } \\
\end{array}$} & \multicolumn{2}{|c|}{ Verb Retrieval } & \multicolumn{2}{|c|}{$\begin{array}{c}\text { Auditory Word } \\
\text { Identification }\end{array}$} & \multicolumn{2}{|c|}{ Verbal STM } & \multicolumn{2}{|c|}{$\begin{array}{c}\begin{array}{c}\text { Articulatory } \\
\text { Motor Planning }\end{array} \\
\end{array}$} & \multicolumn{2}{|c|}{$\begin{array}{c}\text { Visual Word } \\
\text { Identification }\end{array}$} & \multicolumn{2}{|c|}{$\begin{array}{c}\text { Orthographic- } \\
\text { Phonological } \\
\text { Mapping } \\
\end{array}$} & \multicolumn{2}{|c|}{$\begin{array}{c}\text { Orthographic- } \\
\text { Semantic } \\
\text { Mapping } \\
\end{array}$} \\
\hline & & $25.4^{*}$ & $25.08^{*}$ & $4.28^{*}$ & $13.90^{*}$ & 52.13 & $24.89^{*}$ & 34.42 & $29.41^{*}$ & 45.88 & 68.69 & 59.27 & 59.89 & 55.56 & 41.23 & 39.47 & 53.24 & & & & & & \\
\hline $\mathrm{JB}$ & R Posterior & 47.7 & 53.4 & 47.85 & 46.57 & 48.14 & 42.35 & 46.37 & 44.15 & $18.18^{*}$ & $22.53^{*}$ & 58.31 & 49.22 & & & 64.89 & 61.72 & & & & & & \\
\hline $\mathrm{AE}$ & $\begin{array}{l}\text { Multiple } \\
\text { Lesions }\end{array}$ & $28.00^{*}$ & $19.00^{*}$ & $28.68^{*}$ & 43.82 & 75.17 & 58.2 & 26.05 & $1.37^{* *}$ & 40.72 & 55.46 & 66.56 & 76.48 & 41.23 & 55.56 & 36.29 & 42.65 & 41.80 & 47.46 & $2.94 *$ & $15.20^{-}$ & 52.36 & 9.93* \\
\hline $\mathrm{TT}$ & R Frontal & 43.72 & 40.31 & 47.85 & 43.89 & 62.21 & 49.88 & 46.15 & 33.36 & 34.84 & 45.62 & 65.55 & 56.61 & 41.23 & 55.56 & 45.83 & 46.89 & 50.06 & $20.93 *$ & 48.48 & 57.58 & 52.36 & 52.36 \\
\hline $\mathrm{KB}$ & R Frontal & 43.20 & & 47.85 & & 57.78 & & 40.5 & & 61.16 & & 59.03 & & 41.23 & & 35.23 & & 82.77 & & 57.58 & & 52.36 & \\
\hline DAP & L Frontal & 50.81 & & 47.85 & & 54.06 & & $3.00^{*}$ & & 40.36 & 42.77 & 53.23 & & 41.23 & & 41.59 & & 50.74 & & 57.58 & & 52.36 & \\
\hline $\mathrm{TF}$ & L Frontal & 35.91 & 35.88 & 47.85 & 34.51 & 66.48 & 50.9 & $-115^{*}$ & $-20.5^{*}$ & 36.59 & $30.32 *$ & 37.75 & 43.84 & $-1.75^{*}$ & 59.05 & $9.81 *$ & $19.34 *$ & 52.18 & $25.71 *$ & 57.58 & 48.48 & 52.36 & 52.36 \\
\hline TD & L Frontal & 51.68 & & 47.85 & & 55.38 & & 50.05 & & 37.77 & & 54.56 & & $26.91 *$ & & & & 51.63 & & 48.48 & & 52.36 & \\
\hline $\mathrm{DF}$ & R Frontal & 54.46 & 49.11 & 47.85 & 32.96 & 60.87 & 36.93 & 26.3 & $29.51^{*}$ & $15.59^{*}$ & & 41.3 & 42.5 & 55.56 & 41.23 & 32.06 & 34.17 & 48.15 & 66.71 & 39.37 & 48.48 & 52.36 & \\
\hline
\end{tabular}


Preoperatively, $65 \%$ of patients were significantly different to their respective control group in at least one cognitive skill assessed by the BLAST. Furthermore, these patients were more likely to be impaired on one to two cognitive skills than three to five cognitive skills, and no patient was impaired in more than five cognitive skills preoperatively. Specifically (as shown in Figure 5.1), preoperatively $32.5 \%$ of patients were impaired in one cognitive skill, $20 \%$ in two cognitive skills, and 5\% in three, and $2.5 \%$ in four and five cognitive skills.

Postoperatively, $66.7 \%$ of patients were significantly different to controls in at least one cognitive skill assessed by the BLAST. Similar to preoperative performance patients were also most likely to be impaired on one cognitive skill, and there were no patients impaired on more than four cognitive skills. However, postoperatively, patients were less likely to be impaired on two cognitive skills and there was a slight increase in impairment in three and four cognitive skills. Specifically, 39.4\% were impaired on one cognitive skill, $12.1 \%$ on two cognitive skills, $9.1 \%$ on three cognitive and four cognitive skills.

Postoperatively, there were no patients with impairment in more than four cognitive skills (see Figure 5.1). 


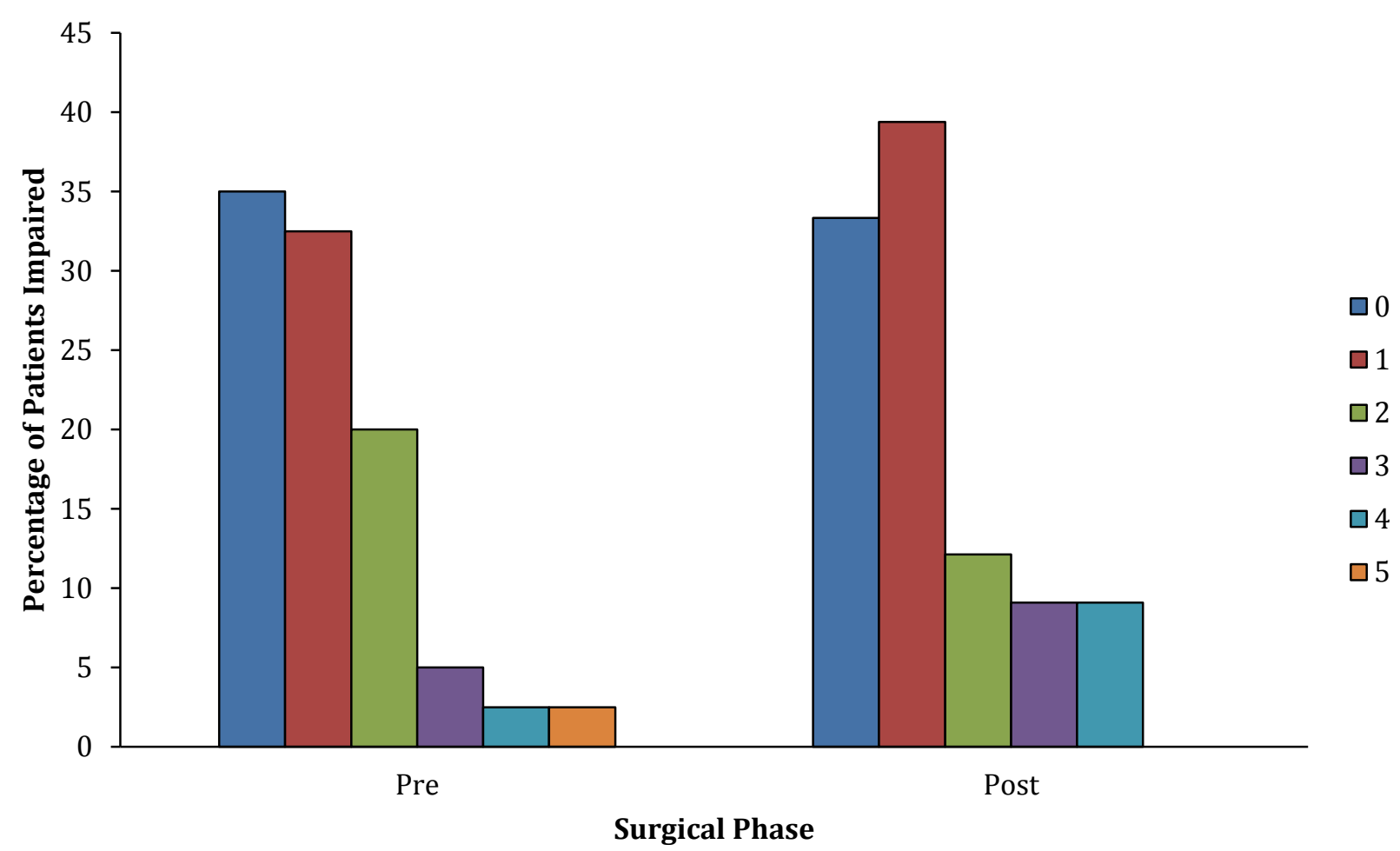

Figure 5.1. The numbers of cognitive skills within the BLAST that patients were significantly different to their respective controls groups on both pre and postoperatively

\section{Specific Cognitive Skill Integrity}

As shown in Figure 5.2, preoperatively, verb retrieval was the cognitive skill most commonly impaired in our tumour sample preoperatively. Specifically, 32.5\% of patients were impaired on this task preoperatively. The other cognitive skills were less sensitive preoperatively: accessing semantic knowledge (12.5\%), lexical selection (7.5\%), goal-driven response selection (15\%), and articulatory-motor planning (8\%). There were no patients impaired in phonological encoding and auditory word recognition preoperatively.

Postoperatively, there was a substantial increase in patients impaired on goal-driven response selection $(37.5 \%)\left(\chi^{2}(1)=4.80, p=.028\right)$. There was also an increase in patients impaired in phonological encoding (9\%) and lexical selection (21.2\%). However, these differences were not statistically significant $\left(\chi^{2}(1)=3.79, p=.051 ; \chi^{2}(1)=3.26, p=.071\right.$ respectively). Conversely, there was actually a decrease in the percentage of patients 
impaired in verb retrieval (15.1\%), although again, this difference was not statistically significant $\left(\chi^{2}(1)=2.93, p=.087\right)$. The percentage of patients impaired in accessing semantic knowledge, auditory word recognition and articulatory-motor planning did not change substantially postoperatively (see Figure 5.2).

Although it appears from Figure 5.2 that visual word identification and orthographical-phonological mapping are particularly likely to be impaired both pre- and postoperatively, inferences drawn in regards to these cognitive skills should be tentative. This is due to the relatively small number of patients that completed the tasks that measure these cognitive skills. This is also true for verbal short-term memory and orthographic-semantic mapping. For this reason, we have decided to not compare performance on these cognitive skills with the other cognitive skills mentioned above, and they have therefore been excluded from further analysis.

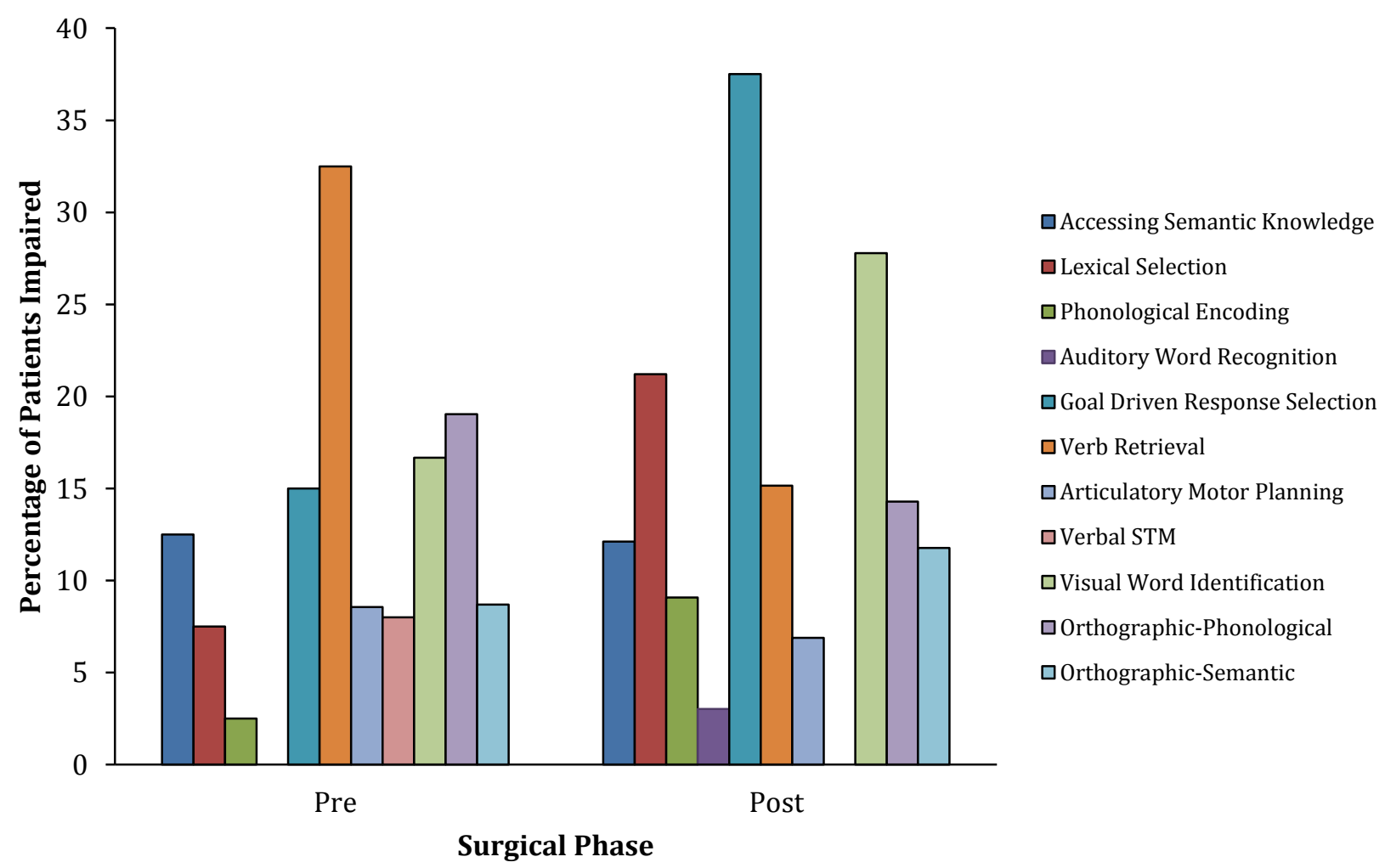

Figure 5.2 The percentage of patients impaired on each cognitive skills assessed by the BLAST both pre and postoperatively 


\section{Cognitive Skill Score by Tumour Neuroanatomy}

Performance on the cognitive skills was further broken down according to the broad anatomical location of the lesion for each patient- left frontal, left posterior, right frontal and right posterior. Visual word recognition, orthographical-phonological mapping, orthographical-semantic mapping, and verbal short-term memory were excluded from this analysis due to the relatively small number of patients who completed the tasks used to derive these cognitive skill scores.

To begin we took a very general approach to the exploration of the implications of tumour localization on cognitive skill performance. To do this we calculated the percentage of patients who were significantly different to their respective control group for each of the four broad anatomical regions. As shown in Figure 5.3, preoperatively, patients with a left posterior lesion were more likely than all other anatomical groups to be impaired on lexical selection and accessing semantic knowledge. In contrast, patients with a left frontal lesion were more likely than all other anatomical groups to be impaired in goal-driven response selection and articulatory-motor planning. This pattern of anatomical specificity persisted postoperatively, plus patients with a left posterior lesion were now more likely to be impaired in phonological encoding than all other anatomical groups. However, caution must be noted in the interpretation of these results due to the small number of patients in the posterior groups. 


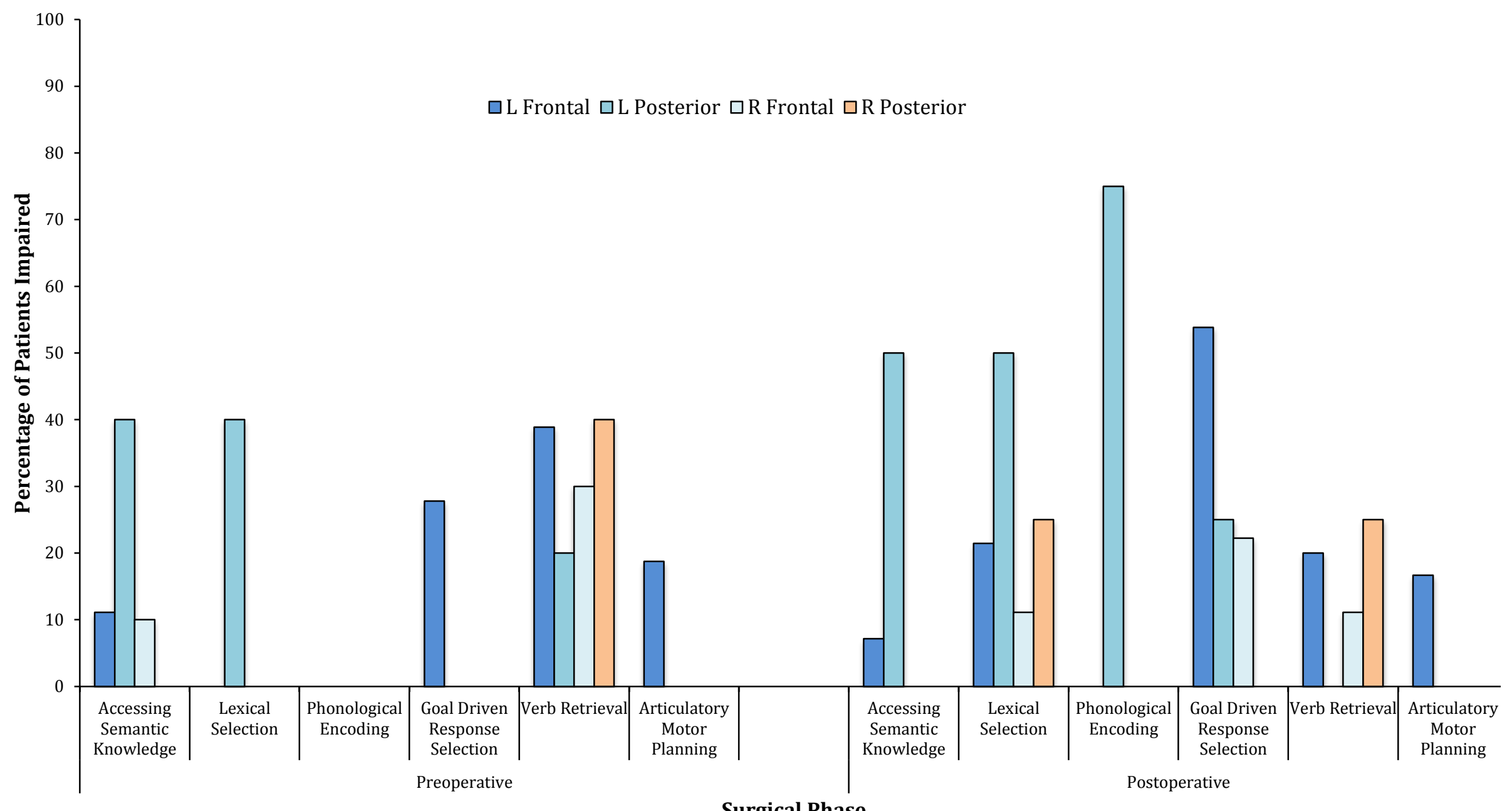

Figure 5.3. The percentage of patients significantly different to their respective control group on each cognitive skill both pre- and postoperatively 
We then conducted an additional analysis by calculating the mean cognitive skill scores for each of the four broad anatomical regions (see Figures 5.4 and 5.5). For these figures, scores were collapsed across the two groups, as there was no significant difference between cognitive skill scores for the two healthy control groups (see Table 5.3). As shown in Figure 5.4, patients with a right frontal and right posterior tumour scored similarly to controls in all cognitive skills examined in BLAST both pre and postoperatively, except for goal-driven response selection. Postoperatively, patients with a right hemisphere lesion (collapsed across other anterior and posterior groups) scored significantly more poorly in this core cognitive skill than controls $(\mathrm{F}(2,52)=7.889, p=.001)$.

Group differences were more evident for the left hemisphere lesion groups (Figure 5.5). Preoperatively, a one-way analysis of variance examining the effect of lesion group (left frontal, right frontal, left posterior, right posterior) on core skill measures revealed a significant effect of lesion group on scores for lexical selection $(\mathrm{F}(3,34)=5.719, p=.003)$, accessing semantic knowledge $(\mathrm{F}(3,34)=6.750, p=.001)$, verb retrieval $(\mathrm{F}(3,34)=3.281$, $p=.033)$, and articulatory motor planning $(\mathrm{F}(3,34)=6.750, p=.001)$. Planned comparisons revealed that lexical selection scores were significantly lower for patients with a left posterior tumour relative to all other groups $(t(34)=-3.920, p<.001)$, and possibly also scores for accessing semantic knowledge $(t(34)=1.700, p=.050)$. Conversely, scores on verb retrieval and articulatory-motor planning were significantly lower for patients with a left frontal lesion than in all other anatomical groups, $(t(34)=3.089, p=.002)$, and $(t(28)=7.780, p=.005)$ respectively. There were no significant effects of group on phonological encoding $(\mathrm{F}(3,34)=0.052, p=.984)$, auditory word recognition $\mathrm{F}(3,34)=1.354, p=.273)$, or goal driven response selection $(\mathrm{F}(3,34)=1.460, p=.243)$.

Postoperatively, a one-way analysis of variance examining the effect of lesion group (left frontal, right frontal, left posterior, right posterior) on cognitive skill measures revealed a 
significant affect of anatomical group on lexical selection $(\mathrm{F}(3,28)=2.979, p=.048)$, accessing semantic knowledge $(\mathrm{F}(3,28)=6.396, p=.003)$, phonological encoding $(\mathrm{F}(3,28)=6.464$, $p=.002)$, and articulatory motor planning $(\mathrm{F}(3,28)=6.396, p=.002)$. A planned comparison analysis found that patients with a left posterior tumour continued to score significantly lower than all other anatomical groups on accessing semantic knowledge $(t(28)=2.636, p=.0075)$ and phonological encoding $(t(28)=-4.062, p<.001)$. For lexical selection, this difference was now approaching significance $(t(28)=-1.685, p=.0502)$. Similarly, those with a left frontal lesion continued to score significantly lower than the other anatomical groups on articulatory motor planning $(t(23)=.4109, p<.001)$. Postoperatively, there was now no significant effect of anatomical group on scores for verb retrieval $(\mathrm{F}(3,28)=1.433, p=.255)$, and auditory word recognition $(\mathrm{F}(3,28)=1.533, p=.229)$. Although it appears from Figure 5.4 that patients with a left frontal lesion had lower scores on goal-driven response selection, there was still not effect of anatomical group on scores for this cognitive skill $(\mathrm{F}(3,28)=2.356, p=.096)$ ( to those obtained preoperatively).

Phonological encoding was the only cognitive skill where there was a significant interaction between tumour group and surgical phase $(\mathrm{F}(3,68)=4.837, p=.004)$. Specifically, patients with a left posterior tumour had significantly lower scores in phonological encoding compared to all other anatomical groups, but only postoperatively. 


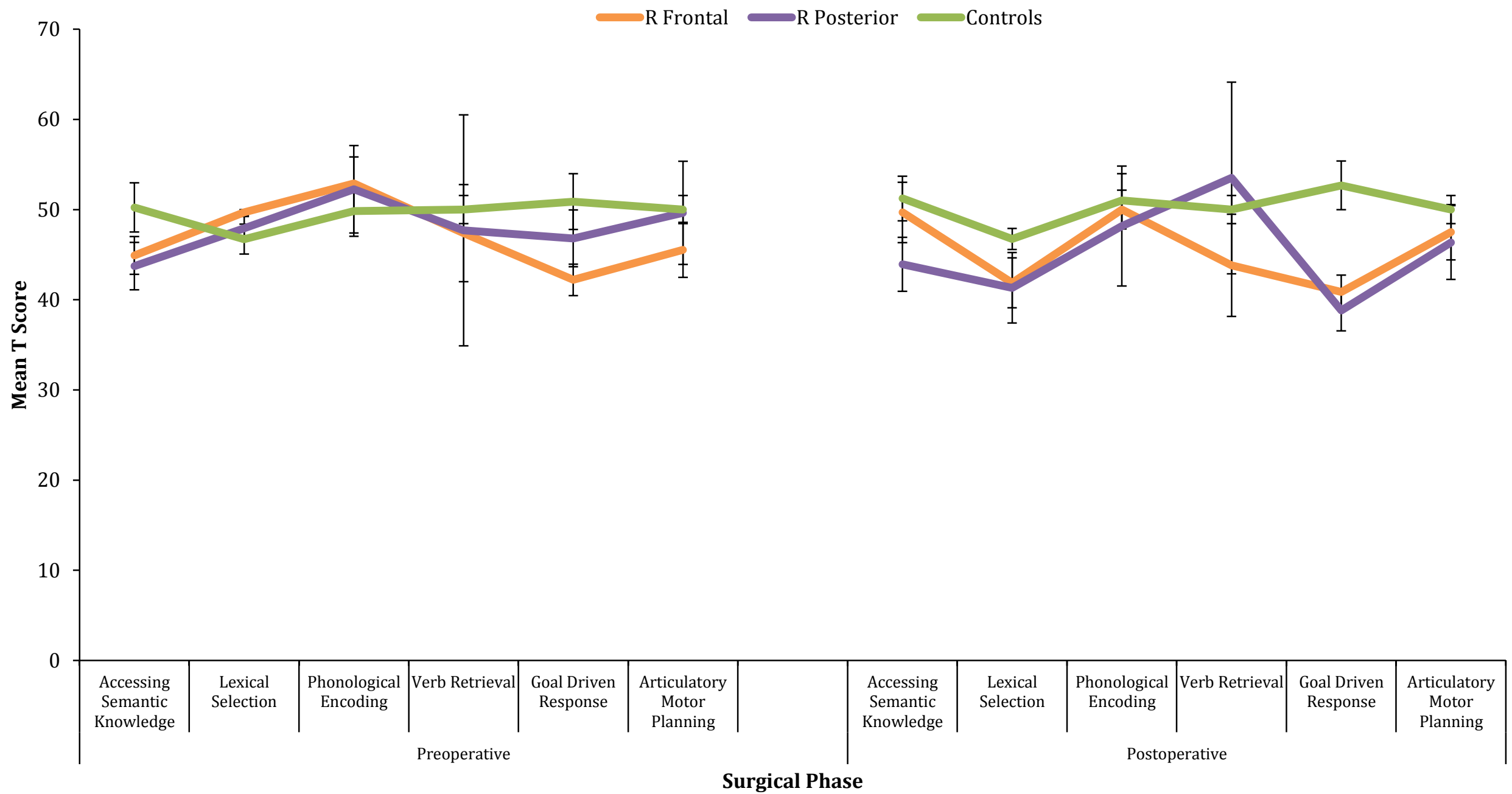

Figure 5.4 The mean T Score for each core cognitive skill for the control group and the right anterior and right posterior group both pre and postoperatively.

Error bars represent standard error of the mean. 


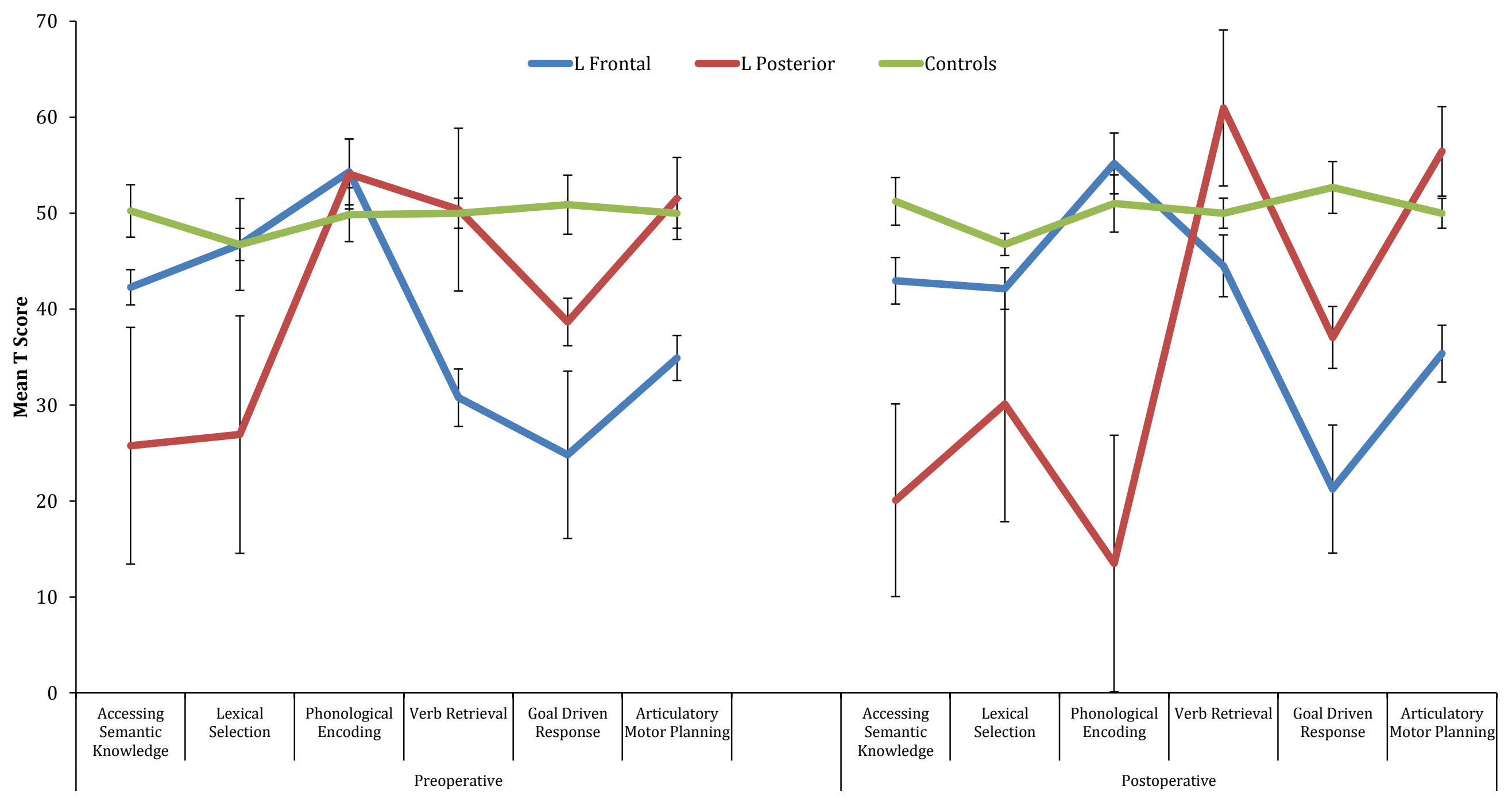

\section{Surgical Phase}

Figure 5.5. The mean T Score for each core cognitive skill for the control group and the left anterior and left posterior group both pre and postoperatively. Error bars represent standard error of the mean. 


\section{Cognitive Skill Score by Tumour Characteristic}

The average scores for high malignant, low malignant tumours and meningiomas, on each of the cognitive skills examined by the BLAST are presented in Table 5.4. One-way ANOVA revealed that there were no significant differences between the these groups on any of the core cognitive skills scores operationalised using the BLAST, neither pre- nor postoperatively.

Table 5.4

The mean T scores for each cognitive skill for high and low malignant tumours, and meningiomas both pre and postoperatively

\begin{tabular}{|c|c|c|c|c|c|c|c|c|}
\hline \multirow[b]{2}{*}{$\begin{array}{l}\text { Cognitive } \\
\text { Skill }\end{array}$} & \multicolumn{5}{|c|}{ Preoperative } & \multicolumn{3}{|c|}{ Postoperative } \\
\hline & High & Low & Meningioma & $\begin{array}{c}p \\
\text { value }\end{array}$ & High & Low & Meningioma & $\begin{array}{c}p \\
\text { value }\end{array}$ \\
\hline $\begin{array}{l}\text { Accessing } \\
\text { Semantic } \\
\text { Knowledge }\end{array}$ & 40.32 & 47.41 & 35.84 & .114 & 39.92 & 45.07 & 42.31 & .802 \\
\hline $\begin{array}{l}\text { Lexical } \\
\text { Selection }\end{array}$ & 38.41 & 46.81 & 45.22 & .154 & 32.79 & 41.59 & 41.96 & .107 \\
\hline $\begin{array}{l}\text { Phonological } \\
\text { Encoding }\end{array}$ & 56.72 & 55.76 & 49.52 & .288 & 47.95 & 47.95 & 51.63 & .582 \\
\hline $\begin{array}{c}\text { Auditory } \\
\text { Word } \\
\text { Recognition }\end{array}$ & 60.61 & 54.24 & 60.24 & .259 & 66.74 & 52.63 & 57.89 & .103 \\
\hline $\begin{array}{c}\text { Verb } \\
\text { Retrieval }\end{array}$ & 42.53 & 43.71 & 37.67 & .794 & 54.43 & 46.13 & 41.41 & .701 \\
\hline $\begin{array}{c}\text { Goal-Driven } \\
\text { Response } \\
\text { Selection }\end{array}$ & 29.46 & 35.18 & 36.89 & .716 & 29.18 & 24.28 & 35.71 & .170 \\
\hline $\begin{array}{l}\text { Articulatory } \\
\text { Motor } \\
\text { Planning }\end{array}$ & 41.67 & 45.90 & 40.96 & .682 & 43.75 & 47.71 & 42.33 & .671 \\
\hline
\end{tabular}

In addition, the average scores for tumour with oedema and tumour without oedema were calculated for each cognitive skill examined by the BLAST (see Table 5.5). 
Independent samples t-tests revealed no significant differences between these two groups on any of the core cognitive skills neither pre- nor postoperatively.

Table 5.5

Average T Scores of the core cognitive skills assessed by the BLAST by oedema presence both pre- and postoperatively

\section{Preoperative}

Yes No $\quad p$ value Yes

\section{Postoperative}

$\begin{array}{llllll}\text { Cognitive Skill } & \text { Yes } & \text { No } & p \text { value } & \text { Yes } & \text { No }\end{array}$

\begin{tabular}{ccccccc}
\hline Accessing Semantic Knowledge & 43.10 & 37.82 & .262 & 43.33 & 40.11 & .598 \\
Lexical Selection & 44.56 & 39.30 & .152 & 40.73 & 37.72 & .123 \\
Phonological Encoding & 52.62 & 53.55 & .831 & 42.00 & 52.20 & .228 \\
Auditory Word Recognition & 57.56 & 59.41 & .960 & 60.51 & 59.41 & .835 \\
$\quad$ & & & & & & \\
Verb Retrieval & 37.13 & 37.23 & .224 & 43.74 & 47.53 & .704 \\
Goal-Driven Response Selection & 26.55 & 38.73 & .987 & 28.06 & 31.21 & .524 \\
Articulatory-Motor Planning & 42.53 & 41.63 & .478 & 44.53 & 42.62 & .595
\end{tabular}

Finally, to assess the effect of tumour volume on each cognitive skill, we analysed correlations between volume $\left(\mathrm{cm}^{3}\right)$ and cognitive skill scores. As shown in Table 5.6, the correlations between tumour volume and cognitive skill were negative for all skills examined by the BLAST preoperatively, however these did not reach statistical significance. Due to the considerable changes that are likely to occur to tumour voume during surgery, this analysis was not computed postoperatively. 
Table 5.6

Correlations between tumour volume and cognitive skill performance both preoperatively

\begin{tabular}{ccc}
\hline Task & $\begin{array}{c}\text { Preoperative } \\
\text { Correlation } \\
\text { Coefficient }\end{array}$ & $\boldsymbol{p}$ value \\
\hline $\begin{array}{c}\text { Accessing Semantic } \\
\text { Knowledge }\end{array}$ & -0.217 & .197 \\
Lexical Selection & -0.151 & .340 \\
$\begin{array}{c}\text { Phonological } \\
\text { Encoding }\end{array}$ & -0.149 & .378 \\
$\begin{array}{c}\text { Auditory Word } \\
\text { Recognition }\end{array}$ & -0.143 & .397 \\
Goal Driven Response \\
Selection & -0.036 & .833 \\
Verb Retrieval & -0.280 & .093 \\
Articulatory Motor & -0.147 & .429 \\
Planning & & \\
\hline
\end{tabular}

\section{General Comments: Cognitive Skills}

In this analysis of the core language skill scores, we found that a large proportion of brain tumour patients scored significantly below healthy controls on at least one core cognitive skill and this was the case both pre- and postoperatively. It is interesting to note that $67.5 \%$ of individuals scored reliably below normal levels on at least one skill measure; this is a much smaller percentage than the $94 \%$ that were found to perform below normal levels on at least one task. One possibility is that overall task performance may be more susceptible to nonlinguistic factors such as fatigue, whereas a core skills measure which are to a large extent measures of the relative performance across different stimulus properties, may be less contaminated by such effects (this issue will be discussed in more detail in the General Discussion). Preoperatively, the most sensitive cognitive skill was verb retrieval, whereas 
postoperatively this was goal-driven response selection. Similar to overall task performance, brain tumour patients were more likely to have a specific cognitive skill deficit, than a more global impairment (i.e. be impaired on one cognitive skill than a number of cognitive skills) both pre and postoperatively.

Consistent with our hypotheses, patients with a left frontal lesion scored significantly more poorly than all other anatomical groups (left posterior, right anterior and right posterior) on verb retrieval and articulatory motor planning both pre and postoperatively. However, scores on verb retrieval were significantly lower in this group only in the preoperative phase. Interestingly, we also found that patients with a right hemisphere tumour scored significantly lower than controls on goal-driven response selection. There are two possible explanations for this unexpected finding. First, it may be the case that this cognitive skill is less lateralised than the other cognitive skills assessed by the BLAST; or second, the measures that we used to operationalise this cognitive skill may recruit more generalised cognitive processes that extend beyond those specifically involved in language (the issue of language specificity will be discussed later in the General Discussion).

We also hypothesised that patients with a left posterior lesion would score significantly more poorly on the posterior cognitive skills lexical selection, phonological encoding, accessing semantic knowledge and auditory word recognition. Although we had a relatively small number of patients within this group, we found partial support for this hypothesis. Patients with a left posterior tumour performed significantly more poorly than all other anatomical groups in lexical selection preoperatively (postoperatively, this effect only approached significance), accessing semantic knowledge (both pre- and postoperatively), and phonological encoding but only postoperatively. However, this anatomical specificity was not evident for phonological encoding preoperatively. Inconsistent with our hypothesis, there was no effect of tumour localisation on auditory word recognition. There was a low 
incidence of impairment in this cognitive skill in any individual both pre- and postoperatively; hence no group differences could be identified. This may be due to poor sensitivity in the measures used to operationalise this cognitive skill, and this will be discussed later in the General Discussion. Additionally, it may also be the case that this simple four-way division of patients into groups is insufficiently sensitive to identify some brain-behaviour relationships, especially those that occur only when a particular key portion of the anatomical region in question is damaged. More fine-grained, voxel levels analyses may be more effective at teasing part brain behaviour relationships in this situation. We also found no significant effect of tumour malignancy or the presence of tumour oedema on any cognitive skill assessed by the BLAST either pre- or postoperatively. Also there was no significant association between tumour volume and cognitive skill score in any other cognitive skill assessed by the BLAST either preoperatively.

Unfortunately, we could not fully investigate the effect of tumour localisation and characteristics on verbal short-term memory, visual word identification and orthographicphonological mapping, due to the limited number of patients assessed on these cognitive skills. Fortunately though, data collection for this project is still ongoing, and it may be possible to do this, once sufficient numbers of patients have been assessed.

Finally, there was very little difference in cognitive skill scores across surgical phases. However there were two exceptions. First, there were significantly more patients impaired on goal-driven response selection postoperatively, compared to preoperatively. It may be the case that this cognitive skill is requiring more controlled language behaviour which is particularly more susceptible to generalised postsurgical effects, such as inflammation or the effects of medication. Second, patients with a left posterior tumour had significantly lower scores on phonological encoding compared to all other anatomical groups postoperatively, but this difference was not evident preoperatively. However, the findings concerning the left 
posterior group in particular need to be treated with caution, due to the small number of patients in the left posterior group.

It should be noted that this cognitive skill assessment constitutes a first attempt at operationalising such skills that will very likely need to be further refined. In order to combine multiple measures into a single entity, we first created standard scores for each individual on each measure, using data from the relevant control group. This method is problematic for measures where controls were at or near ceiling and had very little variation. In this situation, minor variations in performance from a patient compared to controls can result in excessively low $\mathrm{Z}$ scores. The use of multiple measures to operationalise a cognitive skill would hopefully reduce the impact of any particular extreme Z-score measures on the overall cognitive skill score, but would not totally eradicate it. This is particularly salient for tasks such as picture-word verification and repetition where controls perform at or near ceiling.

There are two possible ways that this could be overcome in future. First, a more exhaustive cohort of healthy controls would establish a more representative measure of the variation in scores on each measure. Second, a surgical population could also act as a further control group, for example using a cohort of spinal surgical patients. These patients' scores may be more varied due to nonlinguistic factors that impact on task. We are aware of no studies that have used such a control group to standardise scores. However, it would be extremely interesting to evaluate the validity of this approach in future investigations.

Another concern with the way we have quantified cognitive skills here concerns the methods used to combine multiple standard scores to create a single measure of the skill in question. For example, if we consider the picture-word verification task, accessing semantic knowledge was quantified by subtracting the number of phonological confusions from the number of semantic confusions. The limitation of this approach becomes particularly evident 
when a person performs poorly in one of these conditions but performs similarly to controls in the other condition. An individual with no semantic confusions but a large number of phonological confusions will therefore score above zero on this measure - that is paradoxically, above normal. The rationale behind manipulating the same measure in different ways to assess different cognitive skills was to keep administration time as short as possible. For instances where we have operationalised a cognitive skill in this manner, future research may need to consider independent measures in order to overcome this limitation.

For this analysis, we have taken a very broad categorisation of anatomical localisation. In the next section a fine-grain voxel lesion symptom mapping analysis is conducted to further elucidate the anatomical underpinnings of the various types of core skill deficits. 


\section{Chapter 6: Voxel-Based Lesion Symptom Mapping Analysis}

As part of our exploration of brain tumour patients' linguistic capabilities, one of our objectives was to investigate the BLAST's ability to detect different linguistic profiles based on different neurological profiles. One of the key determinants of this was the localisation of the tumour. Our previous analyses have explored this using a very broad anatomical differentiation (e.g., classification into left frontal, left posterior, right frontal, and right posterior). In order to further explore the effects of tumour localisation on cognitive skill performance, but at a much finer anatomical level, this chapter will report the results of a voxel lesion symptom mapping (VLSM) analysis. VLSM analyses the strength of contribution of each lesioned voxel (the smallest element that can be defined in a threedimensional MRI space) to a specific behaviour of interest, and identifies voxels in which damage is significantly associated with poor scores on the measured behaviour (Baldo, Wilson, \& Dronkers 2012). For each voxel, a statistical test determines whether damage to this particular voxel is associated with significantly poorer scores on the relevant behavioural outcome measure (Bates et al. 2003).

\section{Participants}

\section{Voxel Lesion Symptom Mapping Method}

The participant group in this study consisted of all the individuals with a brain tumour who had completed the BLAST preoperatively, except for patients BD and AEK, whose MRI scans were unavailable. In addition, patients with multiple lesions were not included in this study. Therefore, the preoperative and postoperative analyses contained 36, and 29 patients respectively.

\section{Imaging Methods}

Imaging Acquisition. All patients whose MRI scans were available had undergone a magnetic resonance imaging scan upon admission to the Neurosurgical Ward of Wellington Hospital, prior to their surgery. Whole brain T1-weighted with inversion recovery (FLAIR), 
as well as, in most cases, T2-weighted structural scans were collected at 1.5 Tesla (T1 3D FFE $: \mathrm{TR}=25 \mathrm{~ms}, \mathrm{TE}=4.6 \mathrm{~ms}, \mathrm{FOV}=252 \mathrm{~mm} \times 238 \mathrm{~mm}$, slice thickness $=1 \mathrm{~mm}$; Sagittal 3D FLAIR: $\mathrm{TR}=4800 \mathrm{~ms}, \mathrm{TE}=329 \mathrm{~ms}, \mathrm{TI}=1660 \mathrm{~ms}, \mathrm{FOV}=252 \mathrm{~mm} \times \mathrm{a} 250 \mathrm{~mm}$, slice thickness $=1 \mathrm{~mm}$ ). While T1-weighted images provide high structural definition, T2weighted FLAIR scans are particularly useful for identifying older infarctions and scarred tissue.

Image preparation. For each individual, the lesion was manually drawn onto the participant's own T1-weighted structural preoperative image using MRIcron (Rorden, Karnath, \& Bonilha, 2007; http://www.mccauslandcenter.sc.edu/mricro/mricron/index.html), while, where possible, consulting the T2-weighted FLAIR image as additional guidance. If any uncertainty arouse regarding the exact localization of the tumour boundary, we took a more inclusive approach. This is due to the strong likelihood that a tumour may affect surrounding tissue but lack visualisation to be detected on the MRI scan. Following this, the scans and the lesions were spatially normalised using the segmentation and normalization toolbox in SPM8 (Ashburner et al., 2000; see also http://www.fil.ion.ucl.ac.uk/spm/), implemented in MatLab 8.0 (The Mathworks Inc, Natick, MA). The resultant lesion masks were then overlaid onto a standard template based on healthy elderly individuals (with a mean age of 65 years; Rorden, Bonilha, Fridriksson, Bender, \& Karnath, 2012).

\section{Image Analysis}

Lesion Overlap Analysis. First, to identify brain regions that were commonly damaged in this sample, MRIcron (Rorden, et al., 2007) was used to create a lesion overlay map for all brain tumour patients. This is an important first step because voxels that are never damaged in our population (or only rarely damaged) cannot be assessed at all. In other words, the lesion overlap map provides a rough map of the regions of cortex whose functions can reasonably be assessed using VLSM. 
VLSM Analysis. Two VLSM analyses were run for preoperative and postoperative performance on the BLAST due to differences in sample size. Shallice and colleague's argue that the postsurgical effects salient in postoperative MRI scans, such as swelling and oedma, as well as the difficulties distinguishing between residual tumour and resective tissue sites, makes postoperative MRI scans problematic for interpretation and should be avoided (Buiatti, Skrap \& Shallice, 2012; Buiatti, Mussoni, Toraldo, Skrap \& Shallice, 2011; Campanella, Mondani, Skrap, \& Shallice, 2009; Shallice, Mussoni, D’Agostino \& Skrap, 2010). In support of this, we found these problems particularly evident during an initial pilot test using postoperative MRI scans. We therefore used patients preoperative MRI scans for analyses of both pre- and postoperative scores.

The behavioural measures used for each analysis were the T scores derived for each cognitive skill, as outlined in Table 5.1. Since the behavioural measures were continuous rather than binary, inter-individual differences in the magnitude of the behaviour of interest were maintained, and statistical power maximised (Baldo et al., 2012). In the current VLSM analysis, we used the non-parametric Brunner-Munzel rank order test (Rorden, et al., 2007) provided in NPM (implemented in MRIcron; http://www.mccauslandcenter.sc.edu/mricro/npm/). Further, the analysis was set to discount any voxels damaged in a single participant.

When using VLSM, there is a general risk of increased Type I error, since this method will conduct as many statistical tests as there are voxels in the image. Several studies have recommended the use of False Discovery Rate (FDR) to correct for multiple comparisons over the more conservative Bonferroni correction (e.g., Rorden \& Karnath, 2004), because the latter dramatically increases the probability for Type II errors (Kimberg, Coslett, \& Schwartz, 2007). However, FDR does not control for familywise error rates, so it may be advisable to consider both types of corrections. In the current study, measures are reported 
using both types of corrections. In addition, problems in computing accurate values for small samples have been reported for the Brunner-Munzel rank order test (Medina, Kimberg, Chatterjee, \& Coslett, 2010). In order to account for this, we followed recommendations outlined by Medina, Kimberg, Chatterjee, and Coslett, (2010), and used permutation generated test scores for the Brunner-Munzel test (and a permutation threshold of 1000). Permutation testing involves the random assignment of each participant's behavioural score across each individual voxel. Critical values are calculated for each permutation, "thereby creating a simulation of how extreme observations would be generated under the null hypothesis of no association between lesion site and behavioural score" (Baldo et al., 2012, p. 7). This ensures accurate $Z$ scores are created, even when the sample size is small and the distribution of the data is skewed (Kimberg et al., 2007).

One danger in performing VLSM analyses across a small sample is the lack of statistical power. Therefore, we also present a statistical power map indicating the probability of detecting damage to voxels in different brain regions (the map corresponds to Rudrauf et al.'s (2008) “effective coverage map," which takes the spatial distribution of the lesions in the sample into account. However, the map presented here is based on the Wilcoxon-MannWhitney probability due to the continuous nature of the behavioural data).

Finally, for the overlap images, power map and the VLSM analysis, the resulting lesion maps were examined using the Automated Anatomy Template implemented in MRIcron (Rorden et al., 2007). This template provides the percentage of each specific brain region that is covered by the lesion map, and also the percentage of the lesion map associated with each significant brain region. 


\section{Preoperative Results}

\section{Lesion Overlap Analysis and Power Map}

Figure 6.1 displays the lesion overlap maps and Figure 6.2, displays the lesion power maps for all preoperative tumour patients (separate overlay and lesion maps were conducted pre- and postoperatively due to differences in sample size). Table 6.1 outlines the specific anatomical regions with sufficient power to detect a significant effect at the $p<.05$ false discovery rate. There was insufficient power to detect a significant effect at the familywise $p<.05$ threshold.

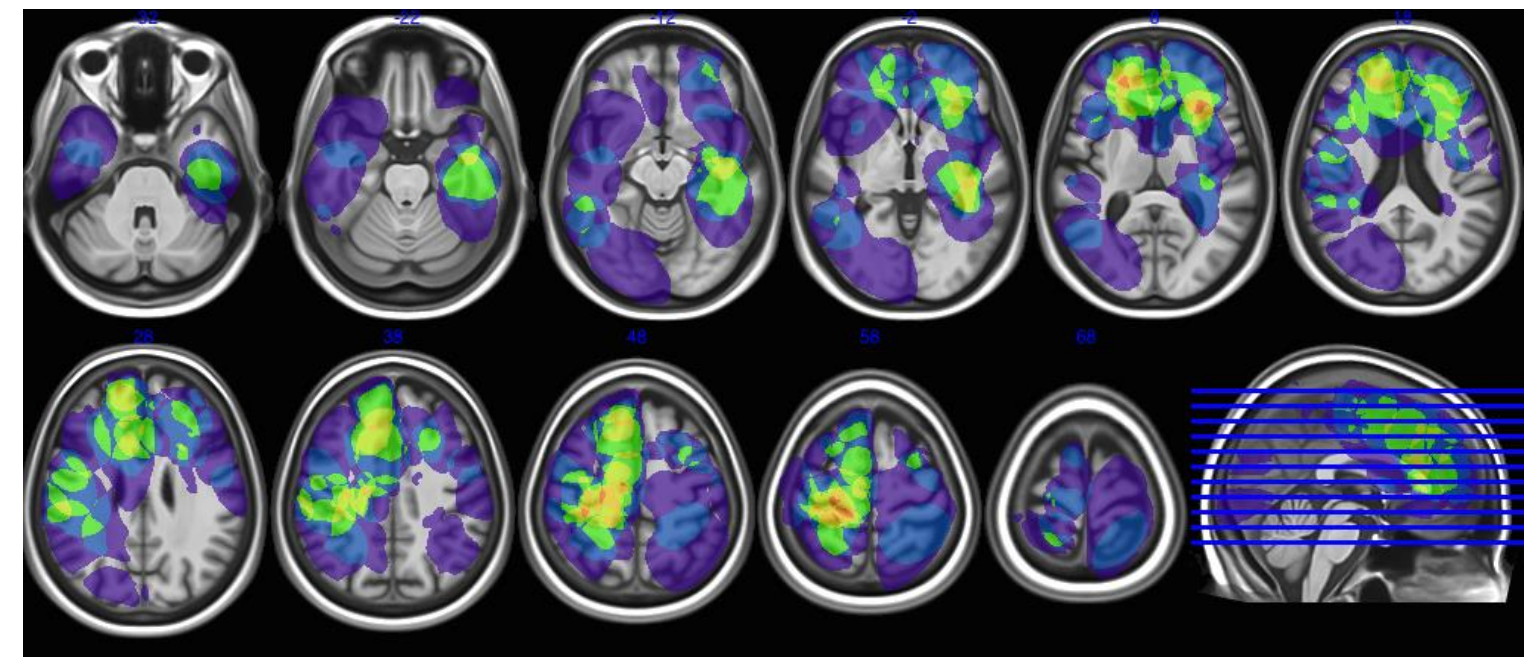

Figure 6.1. Overall Lesion overlap map for tumour patients showing axial slices on a standard Template (Rorden et al. 2012) at MNI Z coordinates $=-32,-22,-12,-2,8,18,28,38$, $48,58,68$. Red $=$ overlap between six individuals; orange $=$ overlap between five individuals; yellow $=$ overalp between four; green $=$ overlap between three individuals; blue = overlap between two individuals; purple = no overlap, lesion is specific to only one individual. 


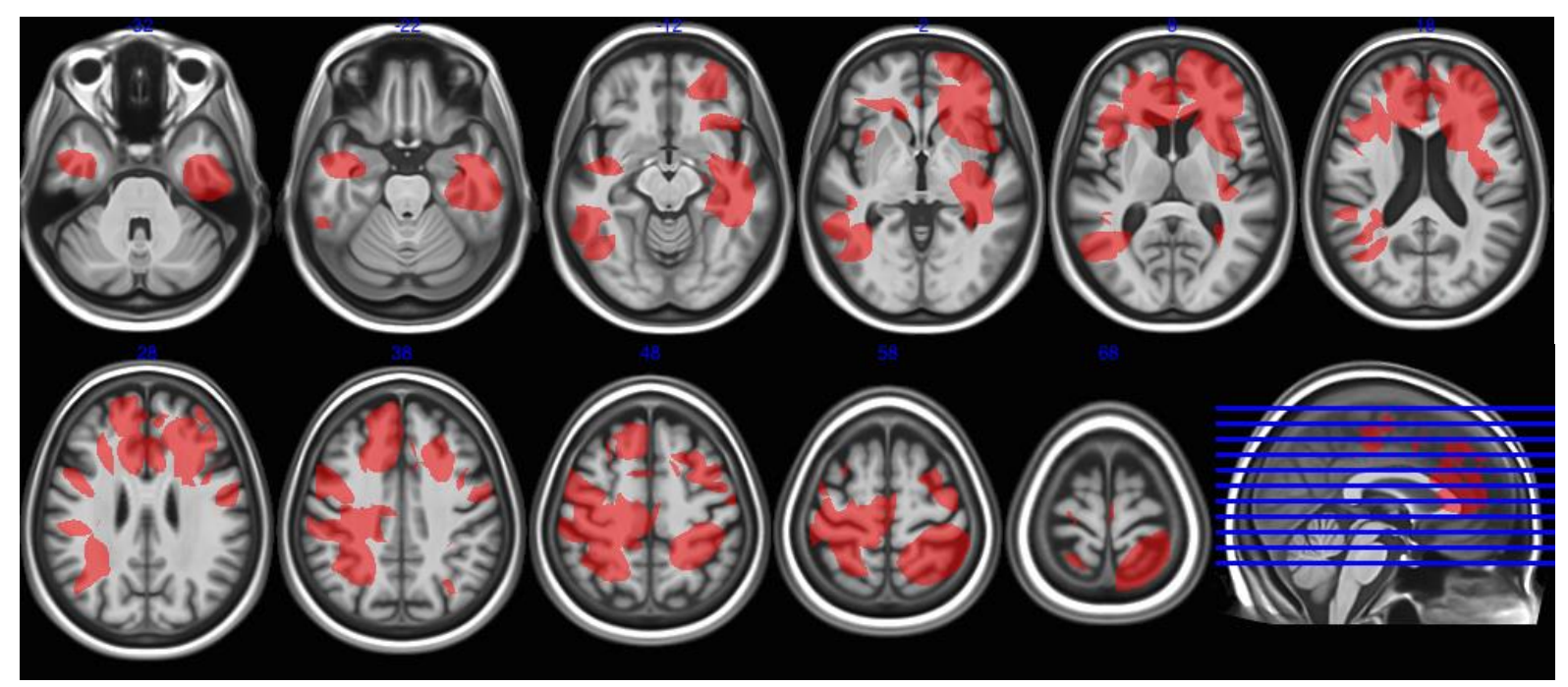

Figure 6.2. Power map for preoperative tumour patients showing axial slices on a standard

Template (Rorden et al., 2012) at MNI Z coordinates =-32, -22, -12, -2, 8, 18, 28, 38, 48, 58,

68. Red $=$ brain regions with sufficient power to detect a significant effect at FDR $p<.05$.

Table 6.1

The percentage of gyri with significant power to detect an effect at the FDR $p<.05$

\begin{tabular}{|c|c|c|c|}
\hline $\begin{array}{c}\text { FDR } \\
\text { Threshold }\end{array}$ & Hemisphere & $\begin{array}{l}\text { Percentage } \\
\text { of Gyri } \\
\text { Covered }\end{array}$ & Brain Region \\
\hline \multirow[t]{4}{*}{$p<.05$} & Left & $<100 \%$ & Anterior cingulate; middle cingulate \\
\hline & & $<60 \%$ & $\begin{array}{l}\text { Pre and post central gyrus; SMA; superior } \\
\text { parietal, and inferior temporal gyrus }\end{array}$ \\
\hline & & $<30 \%$ & $\begin{array}{l}\text { Middle, and inferior frontal gyrus; } \\
\text { supramarginal gyrus; inferior occipital and } \\
\text { middle temporal gyrus }\end{array}$ \\
\hline & & $<10 \%$ & $\begin{array}{l}\text { Angular gyrus; fusiform gyrus; middle } \\
\text { occipital, and inferior temporal gyrus }\end{array}$ \\
\hline \multirow[t]{4}{*}{$p<.05$} & Right & $<100 \%$ & Insula; anterior cingulate \\
\hline & & $<60 \%$ & Fusiform gyrus; inferior temporal \\
\hline & & $<30 \%$ & Middle, and inferior frontal; middle cingulate \\
\hline & & $<10 \%$ & $\begin{array}{l}\text { Precentral; SMA; superior frontal gyrus; } \\
\text { superior, and middle temporal gyrus }\end{array}$ \\
\hline
\end{tabular}




\section{VLSM Results}

Figure 6.3 displays the VLSM maps of significant voxels for preoperative performance in the accessing semantic knowledge, lexical selection, verb retrieval, goaldriven response selection, and articulatory-motor planning. There was no significant brain region associated with behaviour at the family wise permutation level. However a number of significant brain regions were found at the .05 false discovery rate threshold. Table 6.2 provides a detailed description of the percentage of significant lesion map/voxels at each specific brain regions detected at this threshold. A number of left posterior brain regions were significantly associated with performance on accessing semantic knowledge and lexical selection preoperatively. Most notably these were the middle and inferior temporal gyrus.

In contrast, a number of left frontal brain regions were significantly associated with performance on verb retrieval, goal-driven response selection and articulatory-motor planning preoperatively. Most notably, verb retrieval was significantly associated with the left middle cingulate, and the left postcentral gyrus; goal-driven response selection with the left insula and the left middle frontal gyrus; and articulatory-motor planning with the left superior frontal gyrus and left the anterior cingulate. There were no significant voxels at the FDR threshold for preoperative performance on phonological encoding, and auditory word identification. 
Table 6.2

The percentage of significant voxels at each significant brain region at the FDR $p<.05$

threshold for preoperative performance

\begin{tabular}{|c|c|c|}
\hline Cognitive Skill & FDR $p<.05$ & $\begin{array}{c}\text { Number of Significant } \\
\text { Voxels }\end{array}$ \\
\hline \multirow{5}{*}{$\begin{array}{l}\text { Accessing Semantic } \\
\text { Knowledge }\end{array}$} & L Middle Temporal & 37520 \\
\hline & L Inferior Temporal & 16720 \\
\hline & L Inferior Occipital & 11380 \\
\hline & L Middle Occipital & 10770 \\
\hline & L Fusiform & 625 \\
\hline \multirow[t]{5}{*}{ Lexical Selection } & L Middle Temporal & 37520 \\
\hline & L Inferior Temporal & 16720 \\
\hline & L Inferior Occipital & 11380 \\
\hline & L Middle Occipital & 10770 \\
\hline & L Fusiform & 625 \\
\hline \multirow[t]{3}{*}{ Verb Retrieval } & L Middle Cingulate & 635 \\
\hline & L Postcentral Gyrus & 111 \\
\hline & L Precentral Gyrus & 50 \\
\hline \multirow{3}{*}{$\begin{array}{c}\text { Goal Driven Response } \\
\text { Selection }\end{array}$} & L Insula & 329 \\
\hline & L Middle Frontal & 7 \\
\hline & L Inferior Frontal & 6 \\
\hline \multirow{7}{*}{$\begin{array}{c}\text { Articulatory Motor } \\
\text { Planning }\end{array}$} & L Middle Frontal & 43410 \\
\hline & L Superior Frontal & 33410 \\
\hline & L Anterior Cingulate & 13780 \\
\hline & L Middle Cingulate & 892 \\
\hline & L Supplementary Motor Area & 508 \\
\hline & L Inferior Frontal Gyrus & 360 \\
\hline & L Insula & 380 \\
\hline
\end{tabular}


Accessing Semantic

Knowledge

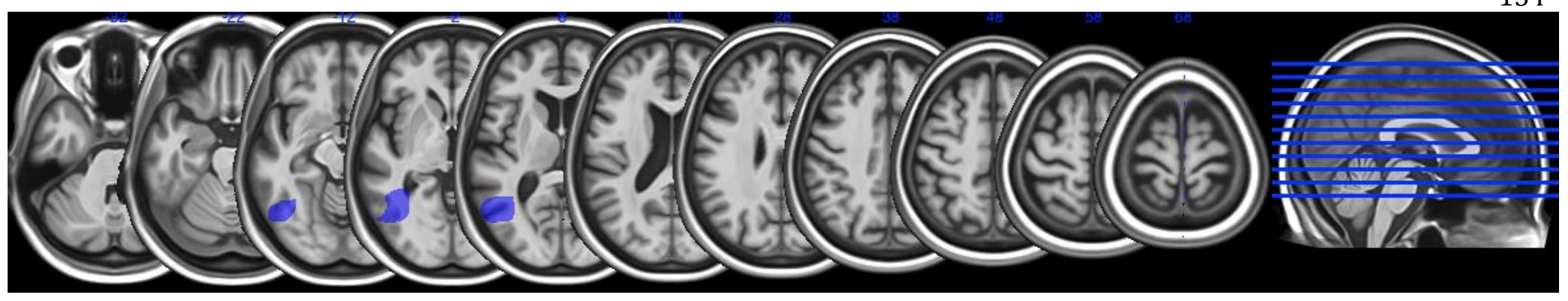

Lexical Selection

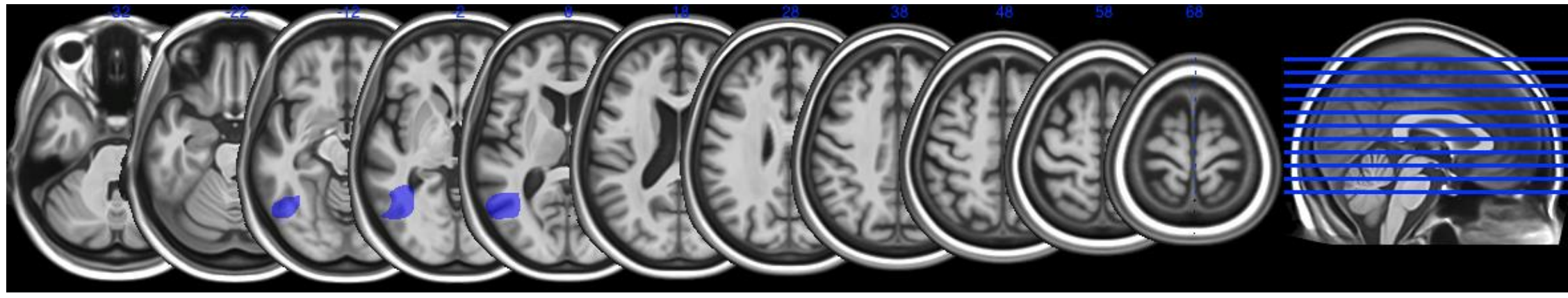

Goal-Driven

Response

Selection

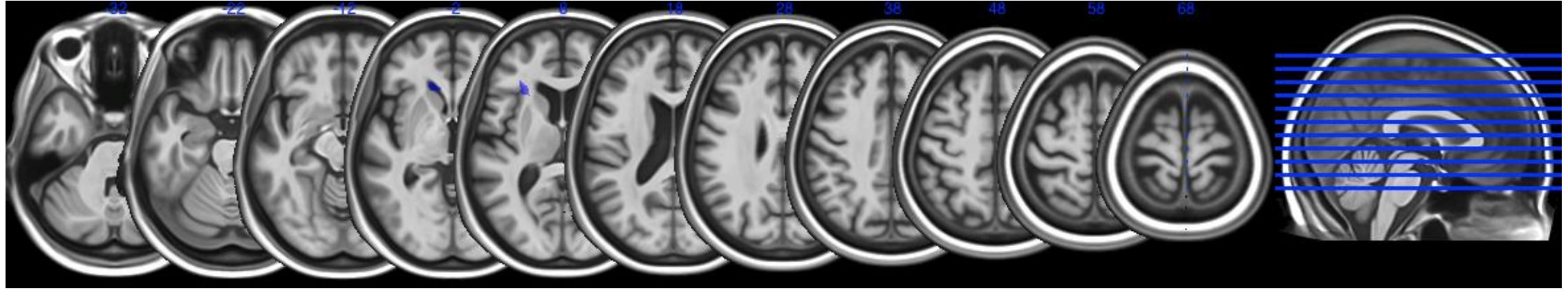


Verb Retrieval

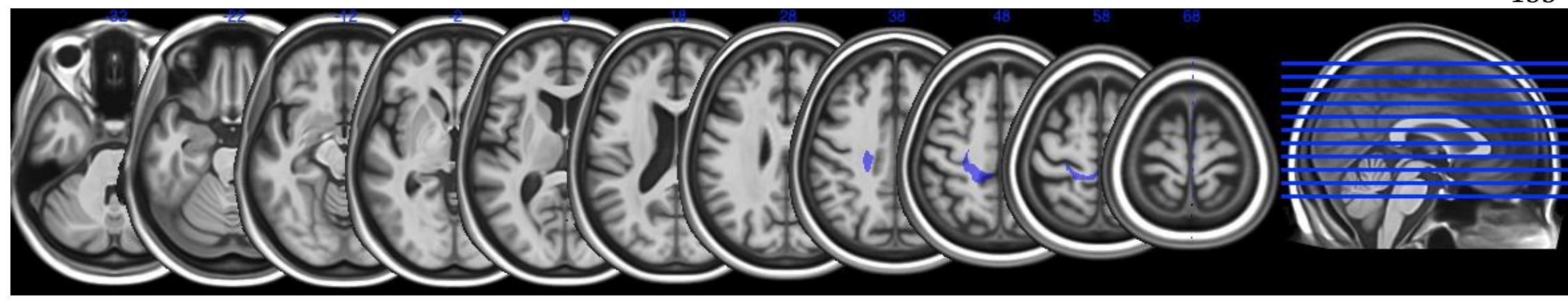

\section{Articulatory-Motor}

Planning

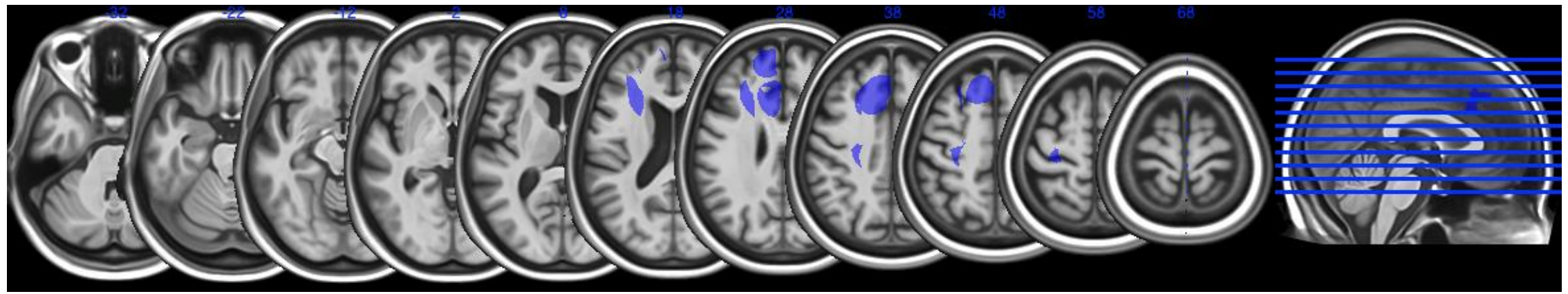

Figure 6.3. VLSM analysis for preoperative performance on accessing semantic knowledge, lexical selection, goal-driven response selection, verb retrieval,

and articulatory-motor planning showing axial slices on a standard Template (Rorden et al., 2012) at MNI Z coordinates =-32, -22, -12, -2, 8, 18, 28, 38, 48,58,

68. Blue $=$ brain regions with sufficient power to detect a significant effect at FDR $p<.05$. There were no significant brain regions at the FDR $p<.01$ threshold. 


\section{Postoperative Results}

\section{Lesion Overlap Analysis and Power Map}

Figure 6.4 displays the lesion overlap maps, and Figure 6.5, displays the lesion power maps for all postoperative tumour patients (separate overlay and lesion maps were conducted pre- and postoperatively due to differences in sample size).Table 6.3 outlines the specific anatomical regions with sufficient power to detect a significant effect at the $p<.05$ false discovery rate. There was insufficient power to detect a significant effect at the family wise $\mathrm{p}<.05$ threshold.

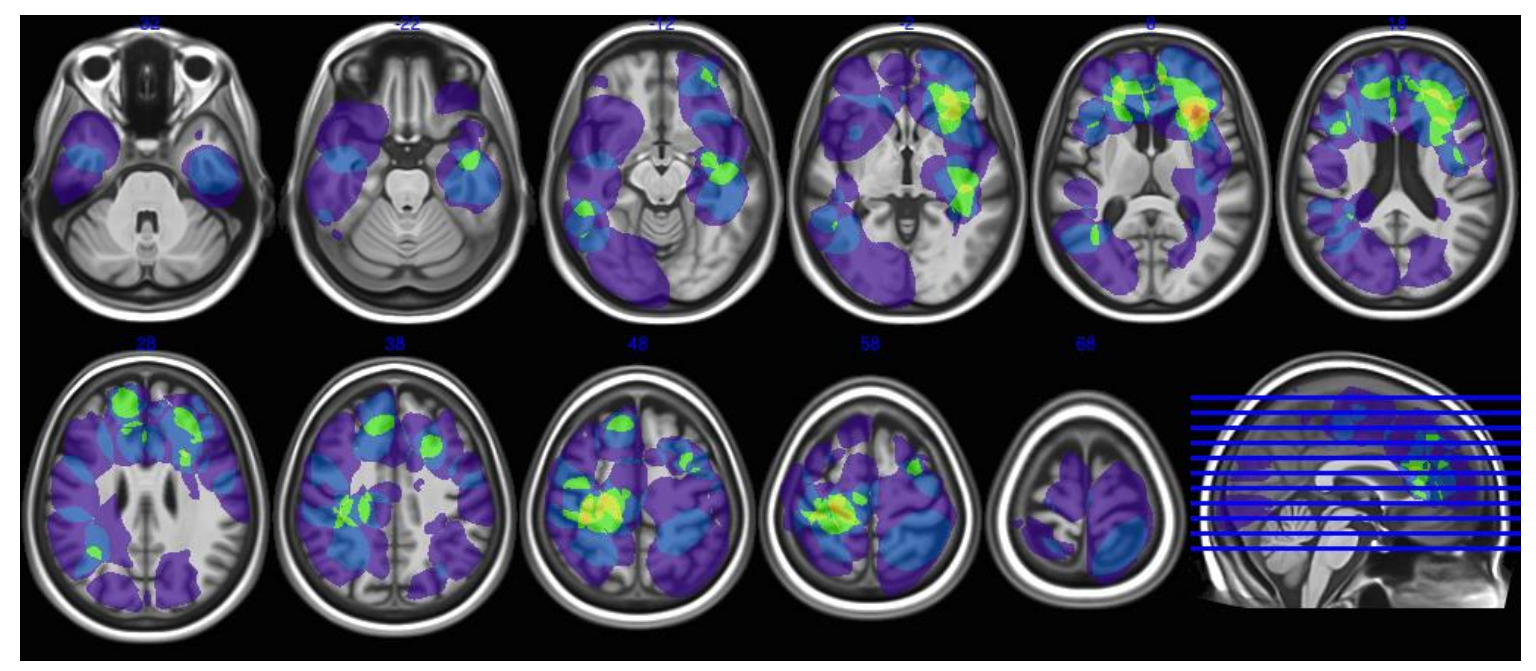

Figure 6.4 Overall Lesion overlap map for tumour patients showing axial slices on a standard Template (Rorden et al. 2012) at MNI Z coordinates =-32, -22, -12, -2, 8, 18, 28, 38, 48, 58, 68. Red = overlap between six individuals; orange = overlap between five individuals; yellow $=$ overalp between four; green $=$ overlap between three individuals; blue $=$ overlap between two individuals; purple $=$ no overlap, lesion is specific to only one individual. 


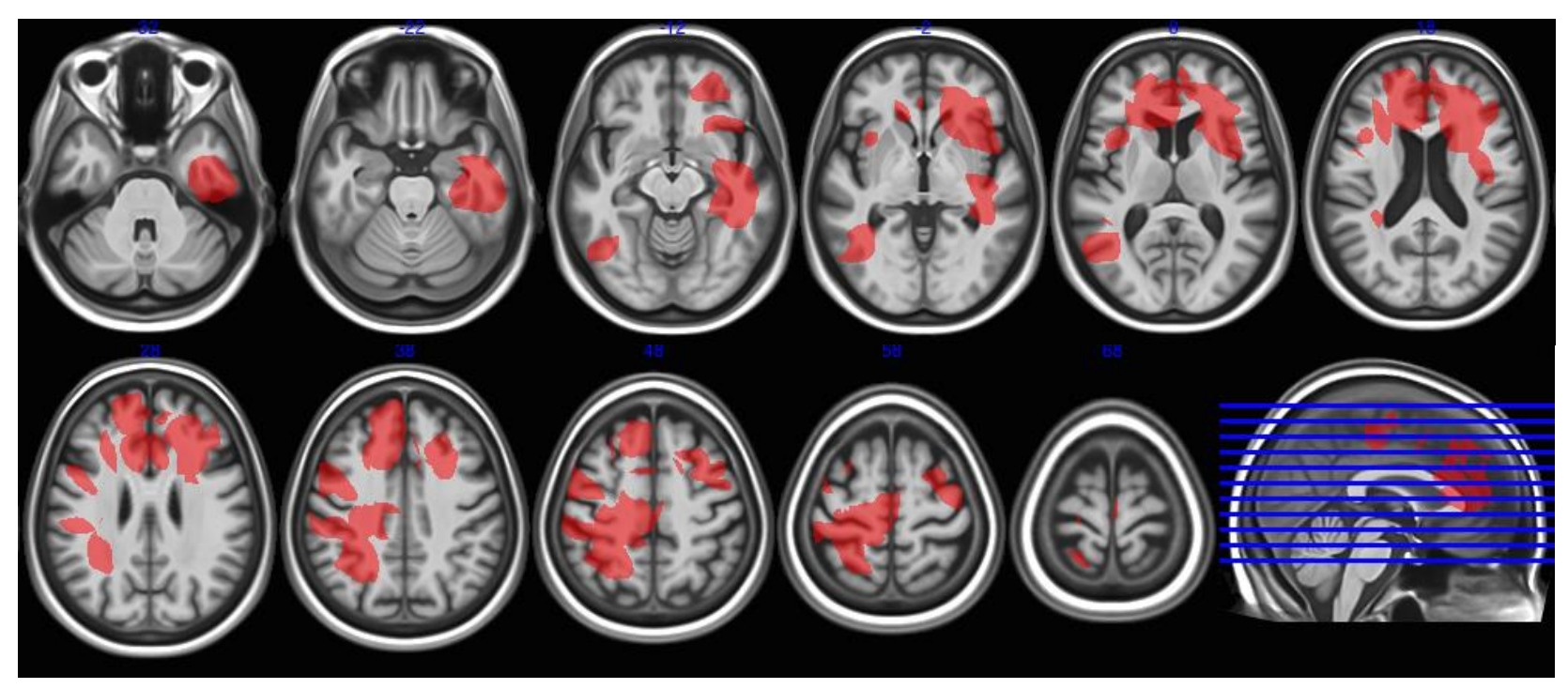

Figure 6.5.Power map for postoperative tumour patients showing axial slices on a standard Template (Rorden et al., 2012) at MNI Z coordinates =-32, -22, -12, -2, 8, 18, 28, 38, 48,58, 68. $\mathrm{Red}=$ brain regions with sufficient power to detect a significant effect at FDR $\mathrm{p}<.05$.

Table 6.3

The percentage of gyri with significant power to detect an effect at the FDR $p<.05$

\begin{tabular}{cccl}
\hline $\begin{array}{c}\text { FDR } \\
\text { Threshold }\end{array}$ & Hemisphere & $\begin{array}{c}\text { Percentage } \\
\text { of Gryi } \\
\text { Covered }\end{array}$ & \\
\hline$p<.05$ & Left & $<100 \%$ & Anterior cingulate \\
& $<60 \%$ & $\begin{array}{l}\text { Precentral gyrus; inferior parietal gyrus; } \\
\text { superior frontal gyrus; middle cingulate }\end{array}$ \\
& $<30 \%$ & $\begin{array}{l}\text { SMA; insula; inferior occipital; postcentral; L } \\
\text { superior parietal; supramarginal gyrus; middle } \\
\text { temporal gyrus }\end{array}$ \\
& $<10 \%$ & $\begin{array}{l}\text { Middle frontal gyrus; middle occipital gyrus, } \\
\text { fusiform gyrus; angular; gyrus inferior } \\
\text { temporal gyrus }\end{array}$ \\
& & Insula \\
& $<100 \%$ & Anterior cingulate \\
& $<60 \%$ & Middle, and inferior frontal gyrus; inferior \\
& & temporal gyrus
\end{tabular}




\begin{tabular}{|c|c|c|c|}
\hline $\begin{array}{c}\text { FDR } \\
\text { Threshold }\end{array}$ & Hemisphere & $\begin{array}{c}\text { Percentage } \\
\text { of Gryi } \\
\text { Covered }\end{array}$ & Brain Region \\
\hline
\end{tabular}

$<10 \% \quad$ Precentral gyrus; SMA; superior, and middle frontal gyrus; middle cingulate; superior, and middle temporal gyrus

SMA $=$ Supplementary Motor Area

\section{VLSM Results}

Figure 6.6 displays the VLSM maps of significant voxels for postoperative performance in the accessing semantic knowledge, phonological encoding, goal-driven response selection, and articulatory-motor planning. There was no significant brain region associated with behaviour at the family wise permutation level. However a number of significant brain regions were found at the false discovery rate threshold $(p<.01$ and $p<.05)$. Table 6.4 provides a detailed description of the percentage of significant lesion map/voxels at each specific brain regions detected at this threshold.. A number of left posterior brain regions were significantly associated with performance on accessing semantic knowledge, and phonological encoding. Most notably the middle and inferior temporal gyrus were significantly associated with performance on phonological encoding at the $p<.01$ FDR threshold. These brain regions were also associated with accessing semantic knowledge but at the $p<.05$ FDR threshold.

In contrast, a number of left frontal brain regions were significantly associated with performance in goal-driven response selection and articulatory motor planning. Most notably, the left superior frontal and left anterior cingulate were significantly associated with performance in goal-driven response selection, but at the $p<.01$ FDR threshold. Also the most notable brain regions significantly associated with articulatory-motor planning were the left superior frontal gyrus and the left middle frontal gyrus, but at the $p<.05$ FDR threshold. There were no significant voxels found at the FDR threshold for postoperative performance on verb retrieval, lexical selection and auditory word recognition. 
Table 6.4

The percentage of significant voxels at each significant brain region at the FDR $p<.05$

threshold for preoperative performance

\begin{tabular}{|c|c|c|c|c|}
\hline Cognitive Skill & FDR $p<.05$ & $\begin{array}{c}\text { Number of } \\
\text { Significant } \\
\text { Voxels }\end{array}$ & FDR $p<.01$ & $\begin{array}{c}\text { Number of } \\
\text { Significant } \\
\text { Voxels }\end{array}$ \\
\hline \multirow{5}{*}{$\begin{array}{c}\text { Accessing } \\
\text { Semantic } \\
\text { Knowledge }\end{array}$} & L Middle Temporal & 43460 & & \\
\hline & L Inferior Temporal & 16720 & & \\
\hline & L Inferior Occipital & 11380 & & \\
\hline & L Middle Occipital & 10780 & & \\
\hline & L Fusiform & 625 & & \\
\hline \multirow{5}{*}{$\begin{array}{c}\text { Phonological } \\
\text { Encoding }\end{array}$} & & & L Middle Temporal & 37520 \\
\hline & & & L Inferior Temporal & 16720 \\
\hline & & & L Inferior Occipital & 11380 \\
\hline & & & L Middle Occipital & 10770 \\
\hline & & & L Fusiform & 625 \\
\hline \multirow{3}{*}{$\begin{array}{c}\text { Goal-Driven } \\
\text { Response } \\
\text { Selection }\end{array}$} & & & L Anterior Cingulate & 17280 \\
\hline & & & L Superior Frontal & 362 \\
\hline & & & L Middle Frontal & 9 \\
\hline \multirow{3}{*}{$\begin{array}{c}\text { Articulatory- } \\
\text { Motor Planning }\end{array}$} & & & L Insula & 235 \\
\hline & & & L Inferior Frontal & 90 \\
\hline & & & L Middle Frontal & 10 \\
\hline
\end{tabular}


Accessing Semantic

Knowledge
Phonological

Encoding

\section{Goal-Driven}

Response

Selection
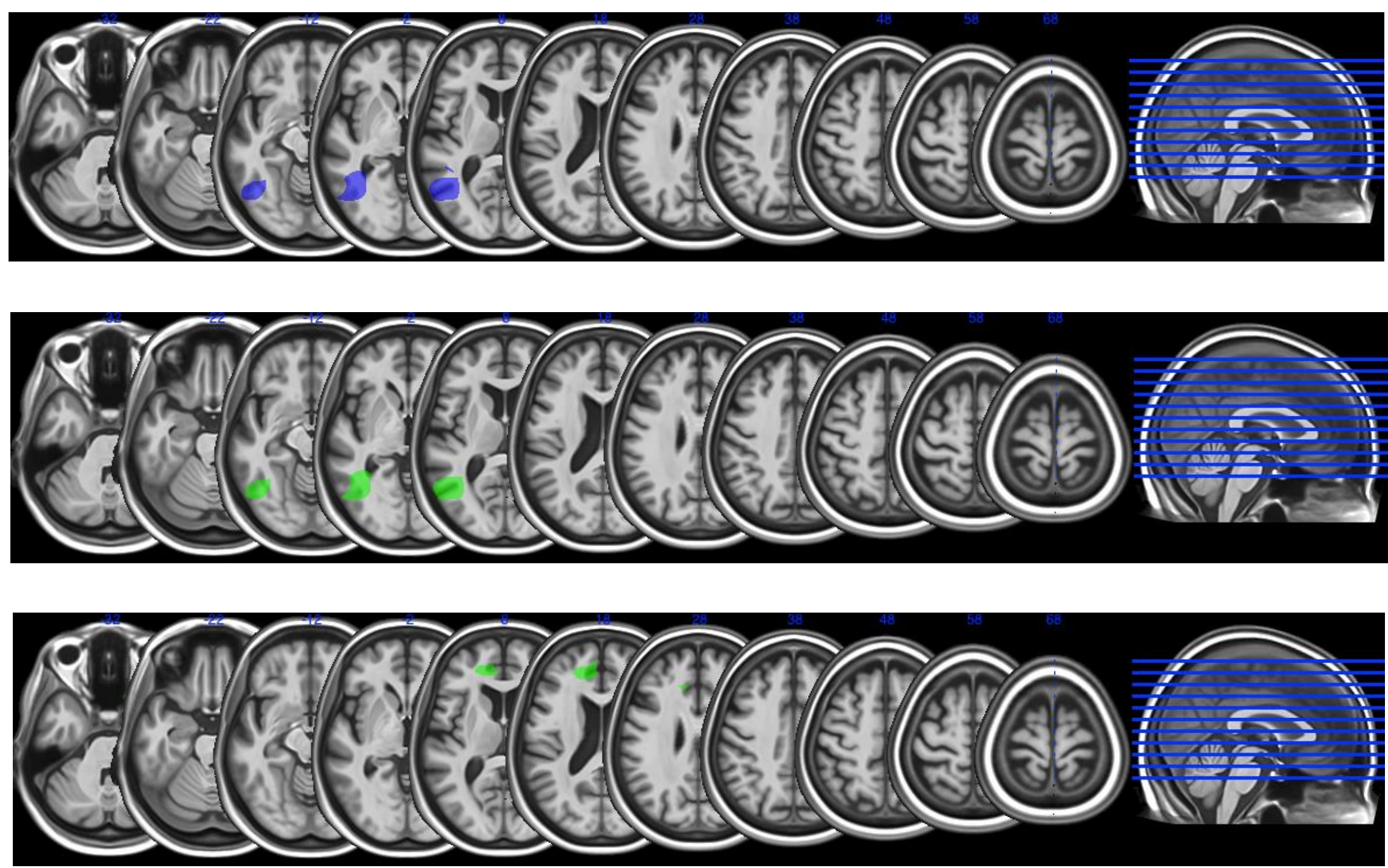


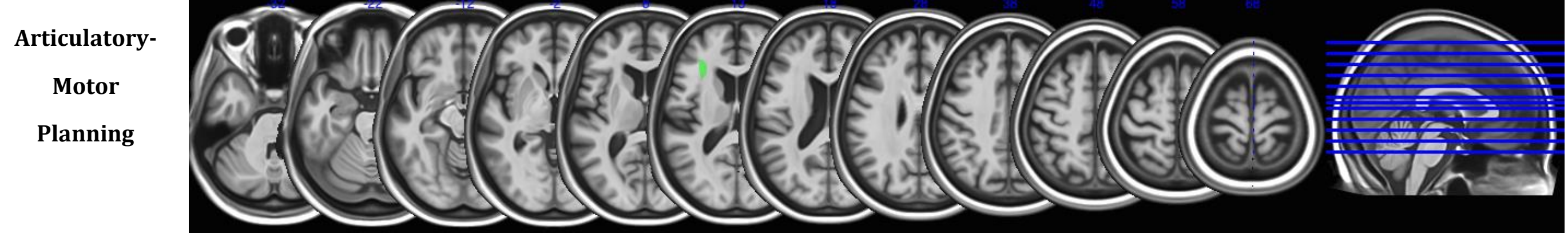

Figure 6.6. VLSM analysis for preoperative performance on accessing semantic knowledge, goal-driven response selection, verb retrieval and articulatory-motor planning showing axial slices on a standard Template (Rorden et al., 2012) at MNI Z coordinates $=-32,-22,-12,-2,8,18,28,38,48,58,68$. Green $=$ brain regions significant at FDR $\mathrm{p}<.01$; blue $=$ brain regions significant at FDR $\mathrm{p}<.05$ 


\section{General Comments: Voxel Lesion Symptom Mapping}

Before discussing the results of the VLSM analysis, it is important to note that we are extremely limited by power when conducting this type of analysis (for discussion see Kimberg, Coslett \& Schwartz, 2007). Only at voxels with high lesion overlap will there be sufficient power to possibly detect a significant association with a behaviour (see Figures 6.2 and 6.5 for power maps). Our sample size is relatively small when compared with VLSM analyses in the literature. Moreover, power maybe further limited by the tremendous variability in the localisation of lesions associated with tumour invasion - most likely considerably more so than with other aetiologies such as stroke or closed head injury, where localisation is constrained by more global factors associated with the aetiology (e.g., distribution of the vascular territory, susceptibility of different brain areas to damage associated with impact). Brain regions with high lesion overlap may therefore be even rarer than in other aetiologies. Any inconsistencies found between our findings and those of the literature should therefore be treated with caution, as this may be due to failure to find a result, rather than no result existing. As previously mentioned, data collection for this project is still ongoing and with a more appropriate sample size to reflect sufficient power, we hope to draw more conclusive inferences about the relationship between specific brain regions and the cognitive skills assessed by the BLAST.

So to summarise, the tentative results obtained from our VLSM analysis support the behavioural results regarding the broad anatomical specialisation of our core cognitive skills. Firstly, in partial support of our first hypothesis that left frontal lesions will be associated with lower scores in goal-driven response selection, articulatory-motor planning and verb retrieval, we found that the voxels significantly associated with these skills were confined to the left frontal lobe (with the exception of verb retrieval tested preoperatively, which failed to yield any significant voxels). In partial support of our second hypothesis that left posterior 
lesions will be associated with lower scores in accessing semantic knowledge, lexical selection and phonological encoding, we found that the voxels significantly associated with these skills were confined to the left temporal and posterior parietal lobes (these effects reached significance preoperatively for accessing semantic knowledge, and lexical selection, and postoperatively for accessing semantic knowledge and phonological encoding ).

Based on our review of the neuropsychological literature, we made some additional more precise predictions regarding the specific brain regions associated with performance on each of the cognitive skills assessed by the BLAST. Our tentative VLSM results do in fact support some of these predictions. Specifically, analyses of preoperative performance showed that low scores in: 1) lexical selection were associated with voxels in the left inferior temporal region; 2) goal-driven response selection in the left inferior frontal gyrus; and 3) articulatory-motor planning in the insula and precentral gyrus.

However, we also found a number of results that were inconsistent with our specific anatomical predictions. For one of our core skills, auditory word recognition, we failed to identify any voxels significantly associated with this skill, either pre- or postoperatively. Such a finding could possibly be due to the poor sensitivity of the measures used to operationalise this cognitive skill. Alternatively, a failure to find an effect for this cognitive skill could be a result of insufficient tumour invasion to the areas associated with this cognitive skill. Further, for several skills, voxels were identified outside the brain areas hypothesised to be associated with that skill. First, accessing semantic knowledge and phonological encoding were associated with significant voxels within the left inferior and middle temporal gyrus, left fusiform gyrus, and the left inferior and middle occipital gyrus (although for phonological encoding, significant effects were only obtained postoperatively). Verb retrieval was associated superior and posterior left frontal regions (including the middle cingulate gyrus, pre- and postcentral gyrus). And finally, in the postoperative analyses goal- 
driven response selection was associated with the left anterior cingulate, and the left middle and superior frontal gyrus.

These inconsistent findings may be a direct reflection of the small size of our sample. More specifically, one or two specific individuals who have large lesions, and who failed on multiple tasks, may create the impression that a large, undifferentiated region of the brain is critical for all those tasks (this is certainly evident in our left posterior group which only contained four patients, and of these patients, two had a relatively large tumour volume). With a larger and more diverse sample, such conflated effects may be differentiated. This further highlights the limits of this methodology when using the current sample and the importance of treating these results tentatively.

In this study, we used the patients' preoperative scans to identify the voxels impaired, but we examined both pre- and postoperative performance. In line with previous researchers (e.g., Buiatti, Skrap \& Shallice 2012), we found that postoperative scans were extremely difficult to read as a result of the impact of the surgical procedure. The marking of a lesion site using these scans would lead to high inaccuracies, and consequently the use of these scans may be potentially problematic. Nevertheless, it is important to note the limitations inherent in the use of preoperative scans for examining postoperative performance. Postoperative lesion sites may be larger, due to the resection of tissue that surrounds the tumour border. Consequently, critical lesion sites that have a direct impact on language functioning could be missed by using only preoperative scans to examine postoperative performance. Finally, it is also important to note that there were differences in power between the preoperative and postoperative analyses. Therefore, it is not possible to directly compare the results of analyses for these two surgical phases. However, despite the power difference results were in fact evident only postoperatively. For example, only significant voxels were detected for phonological encoding during this surgical phase. This finding may 
be due to postsurgical complications adherent postoperatively that impact specially on certain cognitive skills e.g., from tissue resection. This does highlight the importance of conducting postoperative analysis, but caution is needed when deriving conclusions as a result of the surgical phase.

Finally, these results highlight some additional considerations that need to be taken into account when conducting this type of analysis on a brain tumour population. Brain regions will only have significant voxels if they are invaded by the foreign mass and are therefore highlighted during the VLSM. However, this is not to say that the brain tumour does not affect surrounding brain regions. Specifically, a brain tumour can cause compression and displacement on these regions, which can result in a loss of functionality over time. Despite the fact these brain regions lie outside the area identified as lesioned using our method. This may mean that we need to take a more liberal approach when highlighting a brain tumour for a lesion analysis. More extensive research would need to be conducted to determine a standardised approach in order to ensure consistency across future investigations that adopt this methodology. 


\section{Chapter 7: General Discussion}

The objective of this study was to explore the linguistic capabilities of brain tumour patients using a newly developed assessment protocol (the BLAST). A sample of undifferentiated surgical brain tumour patients completed the protocol both pre- and postoperatively. First, we investigated patients' overall performance on each language task within the BLAST; and second, we used their patterns of performance to assess the integrity of a set of core cognitive language skills, derived from current cognitive theories of language. It was found that a substantial proportion of brain tumour patients failed on at least one language task. Further, a large proportion were also significantly impaired in at least one core cognitive skill, suggesting that language deficits are perhaps more prevalent in this population than has previously been assumed. Importantly, there was also a high degree of selectively in patients' performance across tasks: individuals were more likely to be impaired on one or two tasks and core cognitive skills, than to demonstrate a more globalised impairment. Finally, and consistent with the neuropsychological literature, lesion analysis both simple group based analyses and more complex voxel lesion symptom mapping (VLSM) - revealed that the anatomical location of their tumour was a key determiner of how a patient performed on most of the cognitive skills assessed by the BLAST.

The particular approach taken here, of using performance patterns to calculate scores on a set of core cognitive skills, had a number of advantages over more conventional, taskbased assessments. First, the methodology used to operationalise each cognitive skill - which frequently involved comparisons amongst tasks or amongst conditions within the same taskreduced the likelihood that performance would be confounded by nonlinguistic factors (e.g., fatigue), which would be expected to impact on all aspects of performance across the board. Second, a core skills approach appeared to be particularly effective at discriminating patients with different neurological profiles. A broad group analyses based on localisation of the 
lesion found that a number of core cognitive skills were associated with a specific anatomical region. In contrast, such a specialisation was rarely evident when only considering overall task performance. And third, this approach provides a rich linguistic profile whilst adhering to a brief administration time. A brief sensitive language assessment tool such as the BLAST could have potential clinical applications. When administered preoperatively, it can be used to guide surgery possibly lead to fewer postoperative linguistic complications. It can also aid in more specialised and effective postoperative rehabilitation, and in doing so, maximise the person's quality of life. Of course, at an individual level, the BLAST only provides the briefest glimpse of their possible linguistic disability. We hope, however that this work will catalyse additional investigations into language in this population, with the overall objective of designing the most effective language assessment protocol. The following discussion will outline in detail each of the major findings of this investigation, and consider the implications of these from both a clinical and theoretical viewpoint.

\section{The Incidence of Language Impairment in a Brain Tumour Population}

An important feature of this study is that, rather than selecting a subgroup of tumour patients with lesions in regions known to be associated with language, we assessed an undifferentiated sample of tumour surgery patients. The rationale behind this was based on neuropsychological evidence that has implicated an extensive network of cortical regions involved in language (e.g., Damasio et al., 2004; Foundas, 2001; Spitsyna et al., 2006). This aspect of our methodology also enabled us to obtain an estimate of the incidence and profile of impairment in this population more generally. In fact, given the nature of our sample, the incidence of impairments was surprising. Considering first their overall performance on the BLAST subtasks, $94 \%$ and $90 \%$ of preoperative and postoperative patients respectively, were significantly different to their respective healthy control group in at least one task. These rates are similar to, or higher than, those previously reported for test batteries that considered 
a much broader range of cognitive functions. For example, using a standardised psychometric testing procedure that measured various aspects of memory, attention, language and executive function, Tucha and colleagues (2000) found that $91 \%$ of patients with a frontal or temporal tumour were impaired in at least one of these cognitive domains (Tucha, Smely, Preier, \& Lange, 2000). Similarly, using a broad neuropsychological test battery which assessed intellectual function, executive function, memory, language, praxis, and gnosis, Talacchi, Santini, Savazzi and Gerosa (2011) found that 79\% of glioma patients had a deficit in at least one task preoperatively, and 76\% showed a deficit in at least one task postoperatively.

If we consider those studies that have focused just on language function, the reported prevalence of linguistic impairments in previous studies varies widely from study to study; this is due to differences in the definitions of language impairments used, and also the way patients were sampled and/or assessed. Studies that have defined language impairment according to the criteria set out in standard aphasia assessments have reported incidences ranging from $32 \%$ to $63 \%$ in samples of left hemisphere tumour patients (Davie, Hutcheson, Barringer, Weinberg, \& Lewin, 2009; Ilmberger et al., 2008; Recht, McCarthy, O’Donnell, Cohen \& Drachmann, 1989; Whittle, Pringle Taylor, 1998). Those that have defined language impairment based on patient self reports, have reported an incidence rate of $37 \%$ in an undifferentiated sample of high grade gliomas (Thomas, O’Connor \& Ashley, 1995). And finally, those that have defined language impairment as below-normal performance on one or more language tasks within a broader neuropsychological assessment battery have reported, incidence rates ranging from 36 to $67 \%$, in left hemisphere tumour samples (Papagno et al., 2012; Sanai, Mirzadeh \& Berger 2008; Thomas, O’Connor \& Ashley 1995). The approach in the current study was similar to that of the latter neuropsychological studies, we defined a language impairment as significantly impaired performance relative to healthy controls in at 
least one language task. This is a more liberal definition of language impairment and may explain our higher prevalence rates over studies that required a diagnosis of aphasia.

Interestingly though, considering our sample of undifferentiated tumour surgery patients, our incidence rate of language impairment is still higher than studies that have used a similar definition (Papagno et al., 2012; Sanai, Mirzadeh \& Berger 2008; Thomas, O’Connor \& Ashley 1995). The higher incidence of impairment could be due to the BLAST's effectiveness at detecting specific language impairments evident in this population. Each task contained in the BLAST was selected based on its sensitivity at assessing a set of core cognitive skills - skills that are likely to be necessary for everyday language function. Failure in a language task within the BLAST could therefore reflect a fundamental breakdown in at least one core language skill. In support of this, we also found a high incidence of impairment in at least one of the cognitive skills measured by the BLAST. Specifically, we found that preoperatively $65 \%$ of preoperative brain tumour patients, and $67 \%$ of postoperative brain tumour patients were significantly different to their respective control groups in at least one core cognitive skill.

Our current findings add to a growing body of evidence that there is a surprisingly high incidence of language impairment in this population. Although, this finding may not be that surprising, when we consider the pathological mechanisms of tumours compared with other lesions. Tumours are space-occupying lesions and therefore cause compression/displacement of surrounding neural tissue. For that reason, even though a tumour may not be encompassing a "language area", cortical regions crucial for effective language processing may lose their functionality due to the continual growth and subsequent compression effects elicited by the foreign mass nearby (Miceli et al., 2012). This contrasts to the pathological mechanisms of stroke in which neural tissue dysfunction is largely confined to brain regions supplied by the obstructed artery, whilst sparing other cortical regions. 
Our approach to the assessment of language differs from that taken in several previous studies, which have used more conventional aphasia assessments (for review see De White \& Mariën, 2013). These batteries are designed to diagnose language disorders as a result of cerebrovascular accidents, and mainly in the context of more "naturalistic" tasks, such as describing pictures or responding to simple commands or questions. Such an approach may not be optimal for assessing language in brain tumour patients. Consistent with the findings of Davie et al., (2009), as well as Anderson, Damasio, and Tranel (1990), our findings also support the view that language deficits in brain tumour patients are likely to be highly selective, affecting just one or two key cognitive skills, rather than the more globalised impairments that are more common in other aetiologies such as stroke. Conventional aphasia assessments evaluate a range of language skills at once, making it difficult to detect these more selective impairments, which may not, on their own, be sufficient to reduce performance significantly below the level of controls. Indeed, we are not the first researchers to find that more comprehensive neuropsychological test batteries are more effective at identifying language difficulties in this population than conventional aphasia assessments (see Miceli et al., 2012; Påhlson, Ek, Ahlström \& Smits, 2003). We have designed a test battery that assesses specific language skills that are derived from current cognitive theory, but additionally have been found to associated with very specific neural coordinates. This may be more advantageous over conventional aphasia assessments, as it will maximise the likelihood of detecting specific language impairments that appear to be evident in brain tumour patients. The use of various manipulations to specifically assess each cognitive skill may have the further advantage of limiting the patient's ability to behaviourally compensate for the skill in question, an ability that may be highly developed in pathologies involving a slow gradual onset, such as a tumour.

Interestingly, it is relatively uncommon for a patient with a brain tumour to present in 
a clinical setting with a language complaint, even despite their prevalence in this population. Our results suggest that this may not necessarily mean that language function is unaffected. Indeed, patients' awareness of their own cognitive function does not always align with performance measures. For example, Påhlson, Ek, Ahlstrom and Smits (2003) evaluated the difference between 24 low-grade glioma patients' self reports and their performance in a comprehensive neuropsychological assessment. They found a number of significant differences between patients' self-awareness of their cognitive abilities and their actual performance on this assessment. Further, even though the language deficits in tumour patients may be relatively selective, when compared with those of stroke patients, they are nonetheless likely to significantly impact on the patient's everyday functioning. For example, even a highly selective impairment in lexical selection could be responsible for a significant amount of word finding difficulty in everyday speech, and consequently, immense frustration. In fact, there is evidence that even mild linguistic deficits in tumour patients may have significant consequences for their everyday social functioning. In an interesting investigation, Maritz-Gasser, Herbet, Maldonado and Duffau (2012) found that lexical access latency (reaction times using the Boston Naming task) was significantly associated with the ability to return to previous professional activity. Specifically, in patients who had undergone glioma resection, it was found that naming times significantly increased in patients who were unable to return to work, compared to those who had returned to work following surgery. Of course, it may be that in at least some cases, a deficit in picture naming latency reflects a more generalised cognitive slowing, which may impact upon skills beyond language. However, the point still holds though that even very subtle abnormalities on highly specific measures of language function can have real life implications.

Although a high incidence of language impairments was detected in our sample, an alternative explanation could be that at least some of these impair 
ents may reflect a more generalised cognitive impairment, rather than one specific to language functioning. This is important because a number of tasks within the BLAST may recruit additional cognitive resources, not just language. For example, Davidson, Gao, Mason, Winocur and Anderson (2008) argue "one cannot know for certain whether a patient did poorly on verbal fluency because of trouble sustaining attention to the task, poor strategizing, or faulty search of the lexicon, among other reasons" (pp.28). Other examples of cognitive processes that may play a general role in language task performance include response monitoring, sustained attention, and more controversially, the ability to maintain a representation of an internal goal (Bunge, Kahn, Wallis, Miller, \& Wagner, 2003; Crosson et al., 2001; MacPherson, Turner, Bozzali, Cipolotti \& Shallice, 2010; Myachykov \& Posner, 2005; Novick, Kan, Trueswell \& Thompson-Schill, 2009; Shaywitz et al., 2001). In addition, the overall performance of tumour surgery patients may also be affected by a number of further nonlingustic factors: medication, fatigue, psychological stress, postsurgical complications such as inflammation, as well as the generalised compression of brain tissue from the tumour. In the preoperative phase in particular, a patient may be experiencing high levels of anxiety and emotional distress, due to their recent diagnosis and the prospect of a surgical intervention, with its associated risks. Postoperatively, fatigue is likely to be exacerbated, as well as the transient neurological complications associated with the surgery, such as postsurgical oedema/inflammation, transient retraction injury, initial displacement of neural structures, and neuroplastic mechanisms (Bello et al., 2007).

Fortunately though, our approach of operationalising core cognitive skills may actually mitigate the effects of some of these generalised factors on performance. This is because many of the operationalised cognitive skills compare performance across two or more tasks or between different stimulus properties within a task, thereby factoring out some of the more generalised effects. In support of this, we found that the incidence of impairment 
was lower for the core cognitive skills than for overall task performance (cognitive skills: preoperatively $65 \%$, and postoperatively $67 \%$; overall task performance: preoperatively $94 \%$ and postoperatively $90 \%$ ). The finding that a number of cognitive skills are associated with specific anatomical correlates adds weight to our argument that a core skills approach may be more effective than an overall task performance at specifically assessing language capabilities in brain tumour patients.

When considering the issue of how general cognitive factors impact upon language performance, the question arises as to what constitutes "language" function in the first place. Our assessment protocol could be criticised in that it measures skills not conventionally considered to be part of language function - such as those measured on the Stroop task. We would argue that the traditional dichotomy between language and non-language skills may itself need revising. Evidence has emerged recently that skills traditionally thought of as outside the domain of language function, such as working memory, are likely to be emergent proprieties of the language functioning system itself (Buchsbaum \& D’Esposito, 2008; Buchsbaum, Olsen, Koch \& Berman, 2005; Buchsbaum, et al., 2005; Martin \& Saffran, 1997; Martin \& Gupta, 2004). That is, rather than needing working memory to complete language tasks it might be the case that language supports effective working memory. For example, Martin and Saffran (1997) propose a framework in which the mental lexicon is conceptualized as a network containing layers of nodes representing different types of units (e.g. semantics, lexical, and phonological). It is proposed that the constant upward and downward flow of activation between these units can 'refresh' that activation, enabling representations to be mainlined for short periods (see also Martin \& Ayala, 2004; Martin \& Gupta, 2004). According to this theory, this bidirectional flow of activation within the lexical network plays an important role in verbal working memory tasks (see Buchsbaum \& D’Esposito, 2008; Buchsbaum, Olsen, Koch \& Berman, 2005; Buchsbaum, et al., 2005 for an 
alternative proposal that also views verbal working memory as an emergent property of the language system).

Furthermore, it has also been argued that rather than considering cognitive domains such as executive control and language, as distinct entities, it may be the case that there are domain-specific cognitive control processes which operate primarily on language (see Badre, 2008; Badre \& D'Esposito, 2007; Badre, Poldrack, Paré-Blagoev, Insler, \& Wagner, 2005;

Badre \& Wagner, 2007; Hamilton \& Martin 2005). For example, Hamilton and Martin (2005) provide evidence that impairments on Stroop-like inhibitory control tasks can be highly material-specific - for example, an individual can fail on the conventional Stroop but perform completely normally on the nonverbal antisaccade task. They argue that this dissociation provides evidence that there is a distinct component within "executive function" that may be specifically involved in language.

Nevertheless, it is to some extent an empirical question whether the types of skills measured in the BLAST - particularly those associated with anterior cortex - reflect processes that are genuinely specific to language, or whether they reflect the integrity of more domain-general cognitive functions. We aim to address this in a follow-up study, which is currently underway. Brain tumour patients in this study will complete the BLAST, as well as a more extensive neuropsychological assessment, at least three months post surgery. Patients' performance on key BLAST subtasks will be compared with that on a nonverbal analogue of that task (e.g., performance on the Stroop is compared with that an anti-saccade task). We hope that this type of investigation will better determine associations between specific language skills and other more general cognitive processes. 


\section{Effects of Tumour Location and Other Tumour Properties on Performance}

As well as exploring the linguistic capabilities of brain tumour patients generally, we further investigated the effects of tumour location, as well as the characteristics of the tumour (malignancy, the presence of oedema and tumour volume). Interestingly, when considering overall scores on each of our tasks across different subgroups of patients, we found few significant relationships between tumour characteristics (malignancy, oedema and size) and performance. The anatomical location of the tumour only had a significant effect on performance in letter and category fluency. Specifically we found that left posterior patients performed significantly more poorly preoperatively on the category fluency task than all other anatomical groups (but this difference failed to reach significance postoperatively). Conversely, postoperatively, patients with a left frontal lesion performed significantly more poorly on the letter fluency task than all other groups (this difference failed to reach significance preoperatively). These findings are consistent with those of Papagno and colleagues (2012), who found that patients with a left frontal tumour performed more poorly in letter fluency than those with tumours in other regions, both preoperatively and three months postsurgery. Patients with a left temporal tumour also performed more poorly in category fluency than all anatomical groups at least 3 months post surgery (Papagno, Casarotti, Comi, Gallucci, Riva, \& Bello, 2012). These were the only tasks within the BLAST that were found to have anatomical specificity.

Nevertheless, when we consider our cognitive skill measures, greater anatomical specificity emerged. The key results are summarised in Table 7.1. Specifically, both groupbased and VLSM analysis supported an association between left posterior tumours and phonological encoding, lexical selection, and accessing semantic knowledge either pre- or postoperatively, or both. A similar association was found between left frontal tumours and verb retrieval and articulatory-motor planning, and goal-driven response selection, either 
pre- or postoperatively or both. These results are encouraging considering the low power of the anatomical groups in this study. First, they confirm, to some extent, the validity of the measures we used to operationalise these cognitive skills. Second, these results indicate considerable promise for future investigations that adopt this methodology using a more exhaustive sample of brain tumour patients. This is important as studies that use a large sample size will be able to provide complementary data to other lesion modalities. And third, at a clinical level, a test battery that is sensitive to the localisation of the tumour will be advantageous during an awake craniotomy in order to monitor language function most appropriately.

Table 7.1

Key behavioural and VLSM findings from the anatomical localisation analysis of the core cognitive skills both pre- and postoperatively

\begin{tabular}{|c|c|c|c|c|}
\hline \multirow[b]{2}{*}{ Skill } & \multicolumn{2}{|c|}{ Preoperative } & \multicolumn{2}{|c|}{ Postoperative } \\
\hline & Group Effect & VLSM* & Group Effect & VLSM* \\
\hline $\begin{array}{c}\text { Accessing } \\
\text { Semantic } \\
\text { Knowledge }\end{array}$ & Left Posterior & $\begin{array}{c}\text { Middle and } \\
\text { inferior } \\
\text { temporal gyrus } \\
\text { Inferior and } \\
\text { middle occipital } \\
\text { gyrus } \\
\text { Fusiform gyrus }\end{array}$ & Left Posterior & $\begin{array}{c}\text { Middle and } \\
\text { inferior } \\
\text { temporal gyrus } \\
\text { Inferior and } \\
\text { middle occipital } \\
\text { gyrus } \\
\text { Fusiform gyrus }\end{array}$ \\
\hline $\begin{array}{l}\text { Lexical } \\
\text { Selection }\end{array}$ & Left Posterior & $\begin{array}{c}\text { Middle and } \\
\text { inferior } \\
\text { temporal gyrus } \\
\text { Inferior and } \\
\text { middle occipital } \\
\text { gyrus } \\
\text { Fusiform gyrus }\end{array}$ & Left Posterior & $\begin{array}{l}\text { No significant } \\
\text { voxels found }\end{array}$ \\
\hline $\begin{array}{c}\text { Phonological } \\
\text { Encoding }\end{array}$ & $\begin{array}{l}\text { No significant } \\
\text { group effect }\end{array}$ & $\begin{array}{l}\text { No significant } \\
\text { voxels found }\end{array}$ & Left Posterior & $\begin{array}{l}\text { Middle and } \\
\text { inferior } \\
\text { temporal gyrus } \\
\text { Inferior and } \\
\text { middle occipital } \\
\text { gyrus } \\
\text { Fusiform gyrus }\end{array}$ \\
\hline
\end{tabular}




\begin{tabular}{|c|c|c|c|c|}
\hline \multirow[b]{2}{*}{ Skill } & \multicolumn{2}{|c|}{ Preoperative } & \multicolumn{2}{|c|}{ Postoperative } \\
\hline & Group Effect & VLSM* & Skill & Group Effect \\
\hline $\begin{array}{l}\text { Auditory Word } \\
\text { Identification }\end{array}$ & $\begin{array}{l}\text { No significant } \\
\text { group effect }\end{array}$ & $\begin{array}{l}\text { No significant } \\
\text { voxels found }\end{array}$ & $\begin{array}{l}\text { No significant } \\
\text { group effect }\end{array}$ & $\begin{array}{l}\text { No significant } \\
\text { voxels found }\end{array}$ \\
\hline $\begin{array}{l}\text { Goal-Driven } \\
\text { Response } \\
\text { Selection }\end{array}$ & $\begin{array}{l}\text { No significant } \\
\text { group effect but } \\
\text { higher incidence } \\
\text { of impairment } \\
\text { for the left } \\
\text { frontal group }\end{array}$ & $\begin{array}{c}\text { Middle and } \\
\text { inferior frontal } \\
\text { gyrus and insula }\end{array}$ & $\begin{array}{l}\text { No significant } \\
\text { group effect but } \\
\text { higher incidence } \\
\text { of impairment } \\
\text { for the left } \\
\text { frontal group }\end{array}$ & $\begin{array}{l}\text { Superior and } \\
\text { middle frontal, } \\
\text { anterior } \\
\text { cingulate }\end{array}$ \\
\hline Verb Retrieval & Left frontal & $\begin{array}{l}\text { Superior frontal, } \\
\text { pre and } \\
\text { postcentral } \\
\text { gyrus, middle } \\
\text { cingulate and } \\
\text { superior parietal }\end{array}$ & $\begin{array}{l}\text { No significant } \\
\text { effect of group }\end{array}$ & $\begin{array}{l}\text { No significant } \\
\text { voxels found }\end{array}$ \\
\hline $\begin{array}{l}\text { Articulatory- } \\
\text { Motor Planning }\end{array}$ & Left frontal & $\begin{array}{l}\text { Superior, middle } \\
\text { and inferior } \\
\text { frontal gyrus, } \\
\text { middle } \\
\text { cingulate, } \\
\text { supplementary } \\
\text { motor area, } \\
\text { insula }\end{array}$ & Left frontal & $\begin{array}{c}\text { Superior, middle } \\
\text { and inferior } \\
\text { frontal gyrus, } \\
\text { middle } \\
\text { cingulate, } \\
\text { supplementary } \\
\text { motor area, } \\
\text { insula }\end{array}$ \\
\hline $\begin{array}{l}\text { Accessing } \\
\text { Semantic } \\
\text { Knowledge }\end{array}$ & Left Posterior & $\begin{array}{l}\text { Middle and } \\
\text { inferior } \\
\text { temporal gyrus } \\
\text { Inferior and } \\
\text { middle occipital } \\
\text { gyrus } \\
\text { Fusiform gyrus }\end{array}$ & Left Posterior & $\begin{array}{l}\text { Middle and } \\
\text { inferior } \\
\text { temporal gyrus } \\
\text { Inferior and } \\
\text { middle occipital } \\
\text { gyrus } \\
\text { Fusiform gyrus }\end{array}$ \\
\hline
\end{tabular}

* All brain regions are confined to the left hemisphere

\section{Advantages, Limitations, and Suggestions for Future Research}

The findings of this study hold considerable promise for the methodology we have adopted for the operationalisation of these cognitive skills. By adopting a cognitively motivated assessment protocol for the exploration of language in brain tumour patients, we hope to more effectively establish the prevalence and profile of language capabilities in this population. However, we need to ensure firstly that we are appropriately measuring each core 
cognitive skill. These cognitive skills are hypothetical entities, and in order to quantify each skill, we needed to draw upon evidence from a range sources. This is the first investigation that we are aware of that has embarked upon this endeavor, and it is imperative that we validate the methodology. The anatomical correlates of each of our skills provide just one source of validation. Other sources of cross validation would be to give the BLAST to stroke patients with known selective deficits, and to systematically compare our cognitive skills with alternate measures that arguably reflect the same cognitive skill. For example, we could compare scores on our measure of auditory word recognition with those on a phoneme discrimination task or an auditory lexical decision task (Blumstein, Baker \& Goodglass, 1977; Franklin 1989). If performance on these measures are consistent with those of our measure, and our cognitive skill is associated with anatomical regions consistent with the literature, this holds considerable promise that we are in fact measuring the skill most appropriately.

The BLAST was designed primarily as a research tool for examining the incidence of specific cognitively defined language impairments in this population. Because of its brevity, it has the potential to be part of a clinical assessment, perhaps embedded within a more general neuropsychological protocol that examines other aspects of cognitive functioning, such as attention, memory, etc. The finding that a core skills approach is able to determine unique language profiles based on the localisation of the lesion, allows for the possibility of selecting and administering tasks/manipulations that meet the neurological profile of the patient. This is the first step in the development of such an assessment protocol for use with brain tumour patients. We hope that the BLAST will catalyse the development of additional assessments that focus specifically on other cognitive domains e.g. memory, attention or executive functioning. By testing one cognitive domain extensively (in a similar manner to the methodology adopted in this thesis), future researchers may be able to identify those 
measures that are most sensitive at detecting impairment in brain tumour patients, and more specifically based on the localisation of the tumour.

A further potention advantage of an assessment tool of the kind we have developed here, is that it has the capacity to offer new terminology in the diagnosis of language impairment in this population. As previously discussed, the contrasting pathological differences between stroke and tumour lead to very different linguistic profiles. The use of similar labels to encapsulate language impairments across different aetiologies may not accurately capture the linguistic deficits in brain tumour patients. A new conceptualisation of language impairments in brain tumour patients seems needed. This study offers some suggestions as to how we might go about developing such a scheme. For example, if a patient had an impairment on one cognitive skill, then this could translate to a "selective tumourinduced language impairment". The use of consistent and 'tumour specific terminology' could aid in the effective assessment of language throughout all surgical phases, and could further be used to help guide selection of patients for awake craniotomy, and referral for neuropsychological rehabilitation postoperatively. In addition, the use of common terminology in research could help enhance consistency across differing investigations, which may help draw more definitive conclusions surrounding language capabilities and prevalence in this population.

Finally, although the BLAST was designed specifically for the assessment of language in brain tumour patients, the promising results of the current study provides a rationale to examine the BLAST on other etiologies. The assessment of stroke patients at an acute phase, but also those with chronic aphasia would be ideal candidates. If the BLAST were also able to detect specific deficits in core linguistic skills, this would provide a valuable source of information to aid in the most effective rehabilitation for this population. Also from a theoretical viewpoint, the specificity of the BLAST could allow for a better 
understanding of possible differences in linguistic profiles between stroke and brain tumours.

However, there are a number of limitations to the current study, as well as a number of results that were inconsistent with our predictions, which warrant further discussion. An important limitation of the BLAST concerns those functions and abilities that it does not measure. One of the big challenges with neuropsychological assessments in brain tumour patients is establishing the balance between brevity and comprehensiveness. In an ideal world, the best approach would be a comprehensive neuropsychological assessment that assesses multiple cognitive domains, followed by an extensive language assessment including both very specific and more naturalistic language tasks. However, this approach is unlikely to be feasible in most settings. In the current study, participants were tested in the context of a very busy and stressful three to four night stay in an acute hospital, so a longer assessment was not only practically difficult, but also arguably ethically inappropriate. We were unable to assess neuropsychological function more generally, nor were we able to administer a comprehensive aphasia assessment. Limitations of this kind are unlikely to be unique to our particular setting. All assessments need to be designed in order to gather the most important data within the briefest possible time frame. However, it is important to consider the specific consequences of the particular trade-offs we made between brevity and comprehensiveness in the context of the present study.

Furthermore, caution must be noted in the interpretation of language impairments postoperatively. This is due to the array of possible post surgical effects that could temporarily maintain or even exacerbate the incidence of language impairments. This includes postsurgical oedema, transient retraction injury, initial displacement of neural structures, and neuroplastic mechanisms (Bello et al., 2007). In fact there is a growing debate within the literature concerning whether or not postoperative linguistic impairments are in fact transient and are mostly recovered within 3 months (Finch \& Copland, 2014; Wu et al., 
2011), or that the sensitivity of assessment protocol is not accurately detecting these impairments (Ilmberger et al., 2008; Papagno et al., 2012). We are unable to comment on this debate based on the current study. However, in a follow up study we are investigating whether or not a patient's postoperative performance on the BLAST is predictive of their performance at least 3 months post surgery. Our current study provides the springboard in order to enable this future analysis to be performed.

An important concern that could be raised about the BLAST is that it restricts itself to the single word level. Although we assess cognitive skills often considered to be critical for sentence-level processing (e.g., verb retrieval, goal-driven response selection, verbal short term memory), there are currently no stimuli within the BLAST that utilise whole sentences. This may limit its ability to accurately assess some skills specifically involved in sentence level-processing. However, this limitation might not be as critical as it first appears. In fact, recent evidence has emerged that some deficits that appear to selectively affect sentence-level processing are evident in the individual's pattern of performance even in single word tasks (Biegler et al., 2008; Freedman et al., 2004; Hamilton \& Martin, 2005; Raymer \& Kohen, 2006; Schnur et al., 2009; Scott \& Wilshire, 2010; Wilshire \& McCarthy, 2002). For example, Scott and Wilshire (2010) report the case of JHM a nonfluent aphasia with severe sentence level processing deficits, who exhibited significant impairments in the Stroop task. Further both types of deficits appear to have common lesion correlates - most notably, left inferior frontal gyrus (Borovsky, Saygin, Bates \& Dronkers, 2007; Kling 2007; Schnur et al., 2009). We aim to address the precise relationship between performance on our assessment and sentence-level processing in a follow-up study currently underway. In this study, brain tumour patients are assessed using the BLAST at least three months post surgery, and at this time their sentence production and comprehension are assessed using the QPA (Berndt, 2000) the TROG test (Bishop, 2003) respectively. This will allow us to determine if an individual's 
profile on the BLAST is indeed predictive of their sentence-level processing abilities.

Language tasks within the BLAST also rely heavily on concrete stimuli. For that reason, a further limitation is that the BLAST does not contain any task that assesses the processing of more abstract conceptual material. The aphasia literature shows that some patients have specific deficits in the processing of abstract words, but show intact performance in tasks with concrete stimuli (see Franklin, Howard \& Patterson, 1994; 1995; Tyler, Moss \& Jennings, 1995). For that reason, the failure of the BLAST to assess this domain may mean that patients with a specific deficit in processing abstract concepts are missed. Further, Crutch and Warrington (2004) propose a dissociation in the semantic representation of abstract and concrete words. More specifically, the authors suggest that abstract concepts are organised by association (e.g., salute, army, general, respect), whereas concrete words have a categorical organisation (e.g., fruit: apple, strawberry, banana, etc. For further discussion see Shallice \& Cooper, 2013). Although these arguments do not amount to support for a separate and distinct cognitive skill being associated with the processing of these word types - which was the criterion for selection of the skills for the present protocol this issue is nonetheless one worth exploring in future investigations.

It is also important to note that we assessed verb production in a rather unconventional manner. Rather than using action-naming tasks, and comparing performance with that on a set of frequency and age of acquisition matched stimuli in an object-naming task, we used a verb generation task. This decision was made in order to minimise the number of tasks in the protocol; we considered that relevant information about verb production could be learned indirectly from performance on the high-response selection items in this task. However, this task does make rather different cognitive demands. For example, the stimulus noun might "cue" the appropriate verb response, making it much more readily retrievable than in the absence of such a cue (for example, when the stimulus is a pictured 
activity only). Also, there is no carefully matched object-production control task against which to directly compare verb production scores. The relative integrity of noun vs. verb production can only be inferred indirectly, using Z-scores based on control performance on the verb generation and picture object naming tasks, which are not equated in all other relevant respects.

Other potential limitations of our protocol concern the manner in which our various cores skills were operationalised. The core skills we measured in this study were those for which there is considerable supporting evidence in the literature and relatively high agreement concerning their existence. Nevertheless, we made certain arbitrary decisions regarding which aspects of performance we took into account when creating measures of these skills. This issue is particularly salient when considering the complex cognitive processes associated with anterior cortical areas. For example, there is debate within the literature as to whether anterior regions such as the left inferior frontal gyrus operate primarily to drive the lexical retrieval process in line with the current communication goal (a form of "effortful search"), or whether their primary role is to resolve competition (for discussion see Martin \& Cheng, 2006; Thompson-Schill \& Botvinick, 2006). One emerging view is that these processes may be two sides of the same coin (Novick et al., 2009; Novick, Trueswell \& Thompson-Schill, 2005; Novick, Trueswell \& Thompson-Schill, 2010; Thompson-Schill, Bedny \& Goldberg, 2005). Motivated by the latter argument, we operationalised a measure we called goal-driven response selection by combining measures that appear to assess effortful search (letter fluency), and those involved that appear to involve resolution of competition (e.g., congruency effect in the Stroop task). Both sets of cognitive processes have previously been associated with the left inferior frontal gyrus (Baldo, Schwartz, Wilkins \& Dronkers, 2006; Mirman \& Graziano, 2013; Thompson-Schill, D’Esposito, Aguirre, \& Farah, 1997; Tsuchida \& Fellows, 2012; Wagner, Sebastian, Lieb, 
Tüscher, \& Tadić, 2014;).

However, we also found that patients with a right hemisphere tumour also score poorly on goal-driven response selection. It is possible that our decision to combine this wide range of skills into a single score may have reduced the specificity of targeting the core cognitive process specifically involved in language. One way to address this potential problem in future studies is to separate our measures of selection and effortful search, and to determine if their neural correlates are the same or different. If common brain structures were found to be associated with each skill, then this would support our original operationalisation that they both reflect a common cognitive process.

Other potential limitations have arisen not within the theoretical characterisation of the various skills, but rather in the specific tasks that were chosen to measure them. For example, one of the main measures we used to operationalise auditory word identification was accuracy in single word repetition. When considering overall task performance, this task showed poor sensitivity. Given that single word repetition accuracy was at ceiling in most individuals, including the measure might have washed out the lower scores obtained on other measures, such as nonword repetition and picture-word verification involving phonological distractors. This issue of differences in the variability within each of the measures used to operationalize each core skills is a limitation that will need to be addressed, as it can also have a converse effect to that described above. That is, a measure with little variance can led to a large $\mathrm{z}$ score, even when patients have minor variations in performance. This could lead to an inaccurate reduction in the overall core skill measure. A way to overcome this in future would be to adjust the contribution that each measure has to the overall core skill; at present all measures have an equal weighting. Also as mentioned earlier, we strongly advocate that each of our measures is appropriately validated with other sorts of tasks that also assess these cognitive skills. This will not only ensure that we have appropriately conceptualized the skill 
at a theoretical level, but also that the measures we have used to operationalise the skill accurately depict the skill in question.

The final limitation that warrants some consideration arises from the unique difficulties associated with neuroanatomy in brain tumour populations. A debate has recently emerged within the literature concerning whether we can infer brain-behaviour relationships from brain tumour patients. Karnath and Steinbeck (2011) argued that using MRI techniques to infer the representation of human brain functions in tumour patients is "highly problematic" (pp. 1005). First, the authors argued that it is incorrect to treat brain regions as intact simply because they lie outside the tumour border. Evidence to support this view comes from studies suggesting that the diffuse spread of a tumour occurs beyond those areas visualised in an MRI (Burger et al., 1998; Kleihues et al., 2007; as cited in Karnath \& Steinbeck, 2011). Second, or reversely neural function may well be preserved within the tissue that has been infiltrated by a tumour or displaced from that region by the tumour. One final concern raised by these authors is that the brains of individuals with slow growing tumours may be qualitatively different from those of healthy brains as a consequence of functional reorganisation. In reply to these concerns, Shallice and Skrap (2011), acknowledged that many of these criticisms are not limited to the study of brain tumours. For example, the functional localisation of lesions in stroke patients is commonly reported based on standard MRI techniques, which may also fail to detect functional abnormalities in border regions. Instead, Shallice and Skrap (2011) argued that to make a convincing case against the use of tumours for the determinant of brain-behaviour relationships, quantitative evidence would be needed to show that the arguments proposed by Karnath and Steinbeck, (2011) are in an order of magnitude more serious than stroke populations.

We agree that at present there are limitations in using MRI techniques to infer brainbehaviour relationships. All results obtained through this analysis must be treated with 
caution. However, we would argue that despite its potential difficulties, evidence from tumour patients offers a unique insight into human brain functioning. In particular, tumours have a very heterogeneous localisation, and unlike strokes are not confined to regions supplied by the vascular network. They therefore offer a source of evidence that complements other existing sources. Also by examining a range of aetiologies, this in itself may help us to become better aware of the limitations of each, and consequently provide a richer understanding of the language system.

\section{Conclusion}

In conclusion, this study assessed language capabilities in an undifferentiated cohort of surgical brain tumour patients using a novel, cognitively motivated 'core skills' approach. This approach enables the researcher to identify and measure a number of key cognitive abilities that are likely to be necessary for effective language use, while keeping testing time to a minimum. The way in which the core skills were measured makes it unlikely that poor scores were simply due to more general cognitive deficits (such as cognitive slowing, etc.). This investigation found a high incidence of relatively selective language impairments in brain tumour patients both pre- and postoperatively, suggesting that language deficits are perhaps more prevalent in this population than has previously been assumed. It is therefore imperative that we have an effective language assessment tool that is accessible and can be used for this population. Moreover, the various different types of core skills impairments evident in our sample were also associated with distinct neuroanatomical correlates. This not only provides some independent evidence of the conceptual integrity of the core skills approach, but also suggests it may have some value in assessing 'at risk' language skills prior to surgery, and also those possibly impacted by the surgery itself.

Given the brevity and potential neuroanatomical selectively offered by the BLAST, this type of assessment approach has promise as a clinical tool, particularly in preoperative 
clinical assessment, where a brief tool for assessing neuroanatomically specific aspects of language may be of particular value, e.g., identifying candidates for awake craniotomy. This assessment approach also has the potential to be used postoperatively in order to guide cognitive rehabilitation within a time frame where improvements can be maximised. This is the first step in the development of a specific language assessment for brain tumour patients using a cognitively motivated approach. Our next steps are extending our patient pool and further exploring the integrity and validity of the various measures obtained by the BLAST, for example, by comparing them with those obtained using more conventional neuropsychological/aphasia assessments. This initial investigation has provided a useful insight into the linguistic functioning of brain tumour patients, and we hope this can be used to aid in more effective language assessment for this population. 
List of Appendix Tables

Table D.1 .203

Frequency and length of items in version 1 of the picture-naming task in order of appearance Table D.2 .204

Frequency and length of items in version 2 of the picture-naming task in order of appearance Table D.3 .206

Frequency and length of items in version 3 of the picture-naming task in order of appearance Table D.4

Selection strength ratios and frequencies for items in version 1 of the verb generation task in order of appearance

Table D.5 .208

Selection strength ratios and frequencies for items in version 2 of the verb generation task in order of appearance

Table D.6

Frequencies and syllable length for version 1 of the picture-word interference task in order of appearance

Table D.7

Frequencies and syllable length for version 2 of the picture-word interference task in order of appearance

Table D.8

Frequencies and imageability of the single word repetition task in order of appearance

Table D.9

List of items in the nonword repetition task, and their associated actual word in order of appearance. 
Table D.10.

Items, their associated colours and condition in the Stroop task in order of appearance

Table D.11

Items presented in order of appearance in the Articulatory Agility test

Table D.12

Frequency and imageability for section 1 of the reading test in order of appearance

Table D.13.

Frequency, imageability and regularity of section 2 of the reading task in order of appearance

Table D.14

Items presented in order of appearance for the nonword reading task 
Figure A.1. MRI scan of patient SM........................................... 173

Figure A.2. MRI scan of patient PM.......................................... 173

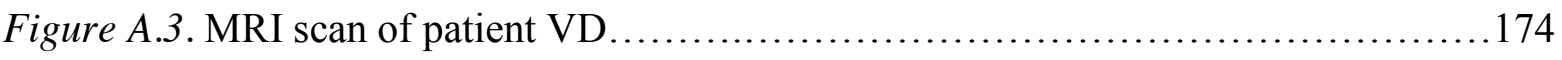

Figure A.4. MRI scan of patient DA.......................................... 174

Figure A.5 MRI scan of EH............................................... 175

Figure A.6. MRI scan of patient RF........................................... 176

Figure A.7. MRI scan of patient SO......................................... 176

Figure A.8. MRI scan of patient CG........................................ 177

Figure A.9. MRI scan of patient EMH......................................... 177

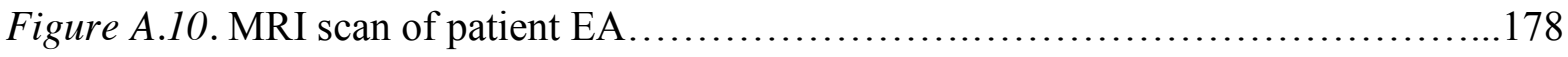

Figure A.11. MRI scan of patient CA.......................................... 178

Figure A.12. MRI scan of patient JM........................................ 179

Figure A.13. MRI scan of patient CR........................................ 179

Figure A.14. MRI scan of patient LC.......................................... 180

Figure A.15. MRI scan of patient DAP .......................................... 181

Figure A.16. MRI Scan of patient MR ......................................... 181

Figure A.17. MRI scan of patient TD............................................ 182

Figure A.18. MRI scan of patient TF.......................................... 182

Figure A.19. MRI scan of patient AVG.......................................... 183

Figure A.20. MRI scan of patient BCA......................................... 184

Figure A.21. MRI scan of patient AM........................................... 185

Figure A.22. MRI scan of patient RJ......................................... 185

Figure A.23. MRI scan of patient LA.......................................... 186

Figure A.24. MRI scan of patient CM........................................... 186 
Figure A.24. MRI Scan of patient LW ......................................... 187

Figure A.25. MRI scan of patient ES............................................ 188

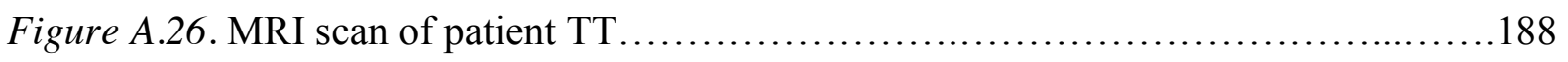

Figure A.27. MRI scan of patient SMC .......................................... 189

Figure A.28. MRI scan of patient DF.............................................

Figure A.29. MRI scan of patient GP .......................................... 190

Figure A.30. MRI scan of patient MRO ........................................... 190

Figure A.31. MRI scan of patient RG..........................................191

Figure A.32. MRI scan of patient TKH...................................... 191

Figure A.33. MRI scan of patient JAS ............................................ 192

Figure A.34. MRI scan of patient AEK ............................................ 192

Figure A.35 MRI scan of patient JB............................................ 193 


\section{Appendix A: Brain Tumour Patients' Case Descriptions}

The following appendix provides a brief case description for each brain tumour patient who participated in this study. The case descriptions have been categorised into the four broad anatomical categories used throughout this thesis. If a patient's MRI scan was available, they are presented here on a standard MRI template (Rorden, et al. 2012) at MNI coordinates $=-22,-12,-2,3,8,19,28,38,48,58,68$.

\section{Left Frontal Group}

\section{SM}

SM is a 49-year-old right-handed woman from a large rural town in the North Island. SM had a history of seizures. Her medical records showed that she experienced her first seizure at the age of 32 , immediately following the birth of her first daughter. Seizures persisted for 10 years, stopping at the age 42 , and leading to cessation of medication for seven years. In July 2009 SM began to experience episodes of a right-sided numbness in her right leg about once a week, and later developed similar episodes of numbness in her face and lips, as well as some confusion and word finding difficulties. A neurological examination in July 2009 found a reduced sensation for light touch in her right arm and leg, but not her face. There was no evidence of motor deficits, and the level of deep tendon reflexes was symmetrical. An MRI conducted at that time revealed a $1 \mathrm{~cm}$ paracentral parasaggital cavernoma in her left hemisphere. The paracentral lobule is located in the distal part of the superior frontal gyrus. SM presented with no significant language deficits. However, she did complain to a speech language therapist of minor word finding difficulties. SM underwent awake craniotomy for the removal of the cavernoma in August 2011. 


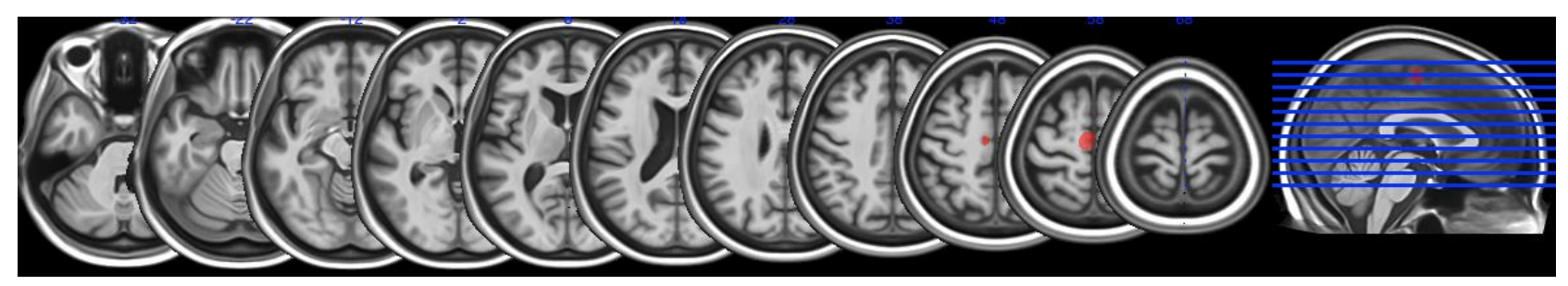

Figure A.1. MRI scan of patient SM

\section{PM}

PM is a 45-year-old right-handed male from suburban Wellington. Medical records show that PM experienced complex partial seizures in of August 2011, and was admitted to the Hutt Hospital Emergency Department. The seizures were described as starting with a funny sensation, following which the patient made strange noises, becoming stiff, biting his tongue and shaking his limbs. These seizures resolved spontaneously. Later the same month, PM was admitted to the Neurosurgery Unit for four days to achieve seizure control. During his stay, PM had four more simple partial seizures. A CT scan of the head demonstrated a hypodense left-sided frontal lesion in the region of the supplementary motor cortex. A further MRI investigation confirmed this as an oligodendroglioma. CT scans are presented in Figure $\mathrm{X}$ and MRI scans in Figure X. PM underwent awake craniotomy for debulking of the tumour in October 2011.

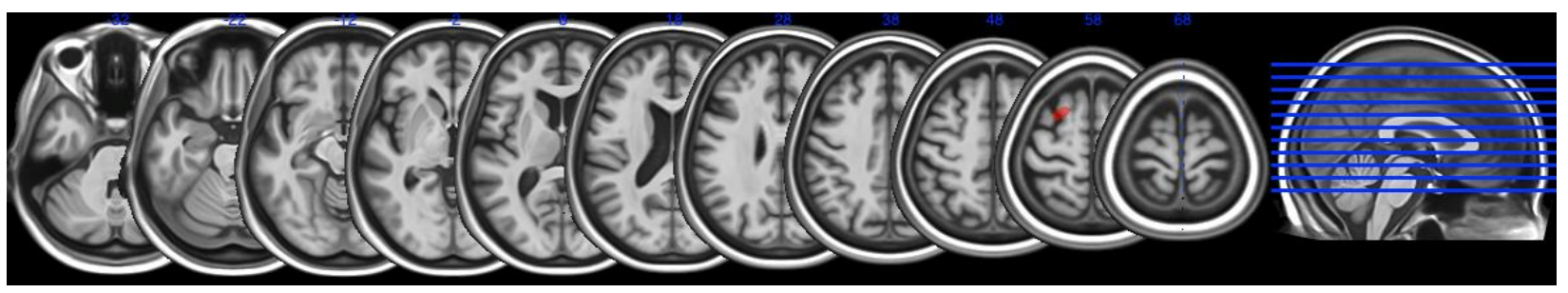

Figure A.2. MRI scan of patient PM

VD

VD is a 56-year-old female from a small rural town in the South Island. In August 2011 she complained of seizures and headaches, and a CT scan showed a large left parietal lesion and another smaller frontal lesion, consistent with cerebral metastases. A CT scan of the chest, abdomen and pelvis showed no evidence of malignant disease. VD underwent a 
macroscopic complete excision of the parietal lesion shortly after. The frontal lesion was not resected. VD made a good recovery postoperatively. On March 2012, VD presented with confusion and hemiparesis, which was relieved by steroids. A frontal craniotomy was subsequently performed to debulk the left frontal glioblastoma in March 2012.

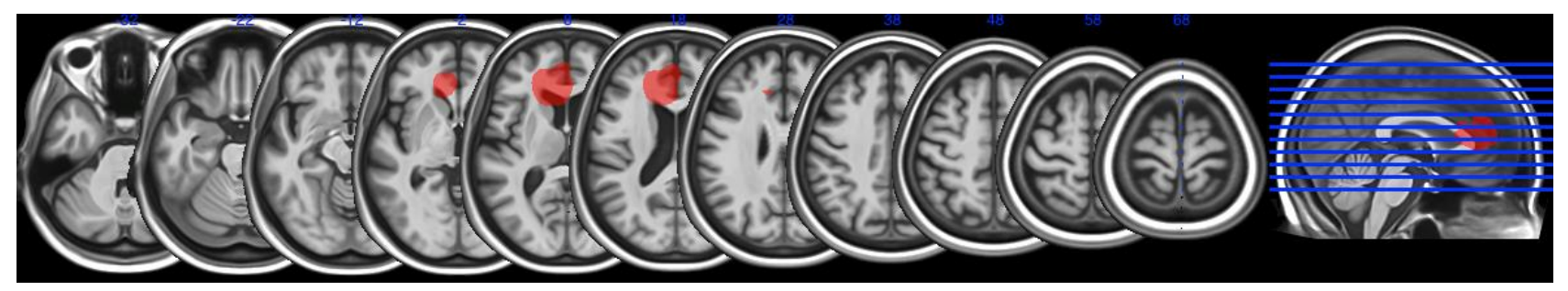

Figure A.3. MRI scan of patient VD

DA

DA is a 33-year-old male from Wellington. In February 2012 DA was examined by neurosurgeon Mr Andrew Parker, who established that there was no neurological deficit. He had no papilloedema or any new cognitive symptoms or changes in limb function. DA reported no symptoms of headache or disturbances of vision, and regarded himself as being normally well. He is an insulin-treated diabetic with low testosterone. DA had a history of leukaemia at age five with a relapse at age eight, where he developed a transient hemiparesis. This required total body radiation and a bone marrow transplant. An MRI performed at this time revealed a relatively large left frontal parasagittal meningioma. Surgery to debulk the tumour was undertaken in April 2012.

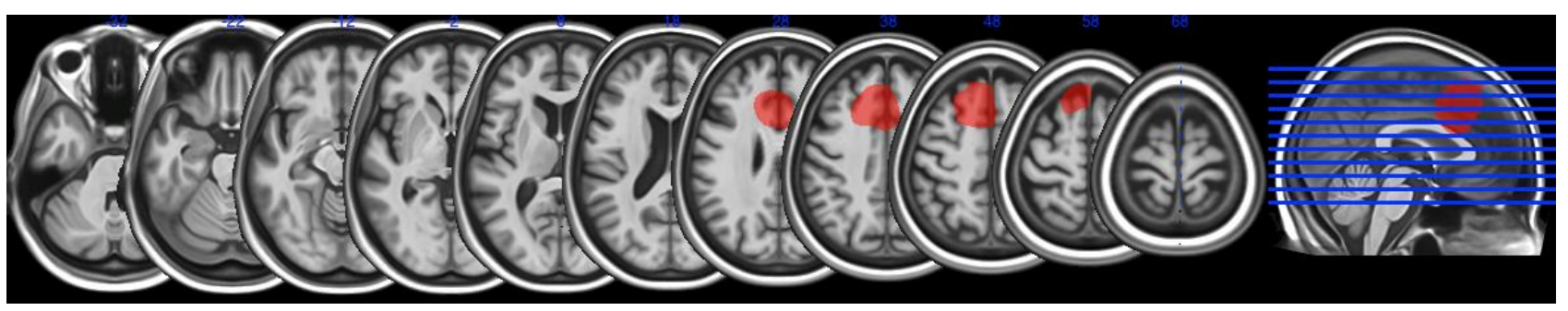

Figure A.4. MRI scan of patient DA 


\section{EH}

EH is a 39-year-old female from a small rural town in the North Island. When seen at her local hospital in November 2011, EH complained of frequent seizures during the past three months. EH indicated that overall she had suffered from five grand mal seizures, the last of these being approximately four weeks prior (there was a decrease in the frequency of these seizures, which coincided with Epilim administration). EH also complained of a left retro-occipital headache, which had been present for several months. There was no particular pattern to this pain, which could come on at almost any time. Neurological examination by Mr Andrew Parker revealed that her vision, cognitive function and motor function were all normal. A CT scan and subsequent MRI demonstrated an enhancing extra-axial left frontal lesion attached to the convexity dura and extending into the falx. There appeared to be some erosion of the frontal bone, but the tumour margins did not overlap with the frontal sinus. There also appeared to be significant sinus disease both in the frontal and maxillary sinuses, more prominent on the left. There was also thickened dural tail - all appearances consistent with a $3 \mathrm{~cm}$ meningioma. The tumour was diagnosed not long after EH had given birth, so it was decided to defer EH's surgery until her child had grown and was a little more robust.

Resection of the meningioma occurred in May 2012.

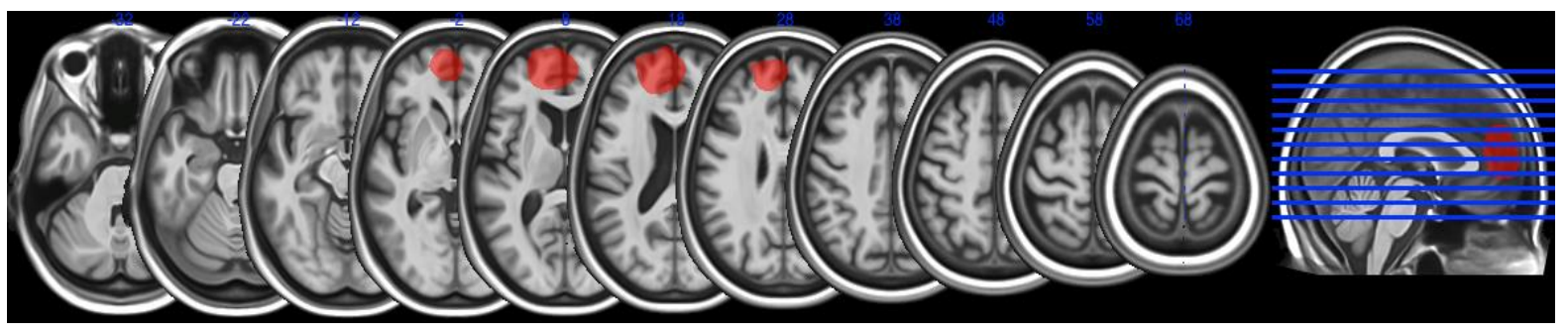

Figure A.5. MRI scan of EH

RF

$\mathrm{RF}$ is a 65-year-old male from a small costal town in the South Island. RF was admitted to Wellington Hospital in July 2012, two weeks after he had experienced a partial seizure at home that caused a loss of consciousness. Subsequent medical examination 
revealed that RF had a mass in the left parafalcine, posterior frontal lobe that was consistent with a meningioma. RF's surgery was performed in early July 2012.

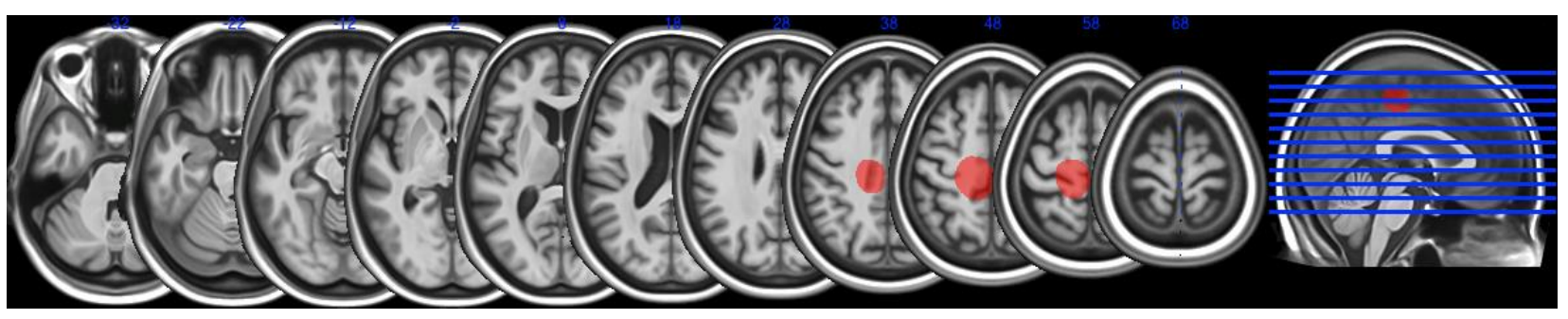

Figure A.6. MRI scan of patient RF

SO

SO is a 58-year-old man from suburban Wellington. He had experienced a three-week history of deteriorating right upper limb function. An MRI scan in August revealed an irregular heterogeneously enhancing tumour just right to the anterior motor strip in the left hemisphere. Craniotomy for debulking the tumour occurred in late August 2012.

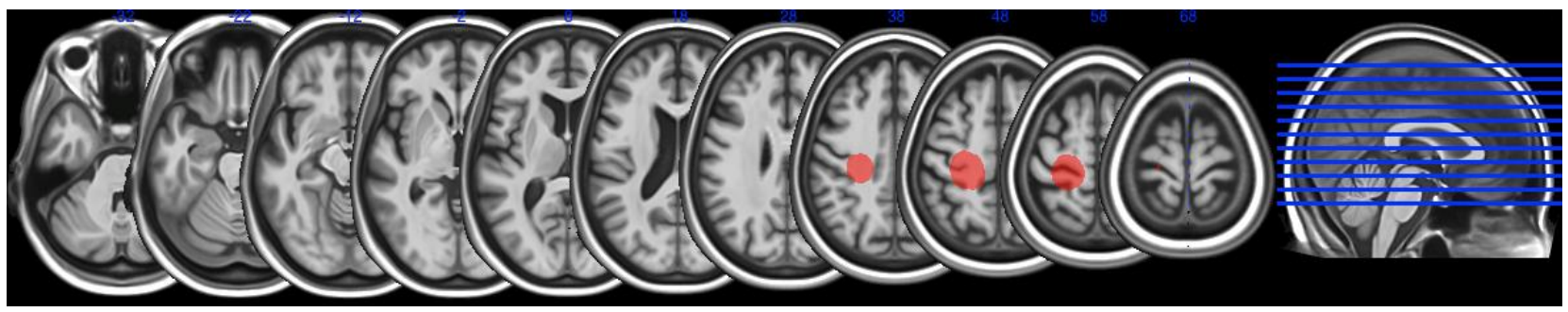

Figure A.7. MRI scan of patient SO

CG

CG is a 46-year-old female from a small rural town in the North Island. CG had a history of breast cancer three years ago, including a bilateral mastectomy, and sustained a left frontoparietal haemorrhage. At that point, she presented with a dense right-sided hemiplegia, but this was improving. Of note she also had a previous lung lesion that was $1.4 \mathrm{~cm}$ in size, which had increased to $6 \mathrm{~cm}$ in size. Initially it was planned to perform serial MRI scans for her whilst at the rural hospital, but unfortunately she sustained another large left posterior/frontal haemorrhage including intraventricular extension with a dense right-sided hemiplegia. She was subsequently transferred to Wellington Hospital for surgery for 
resection of the clot underlying the posterior/frontal tumour. Surgery occurred in November 2012.

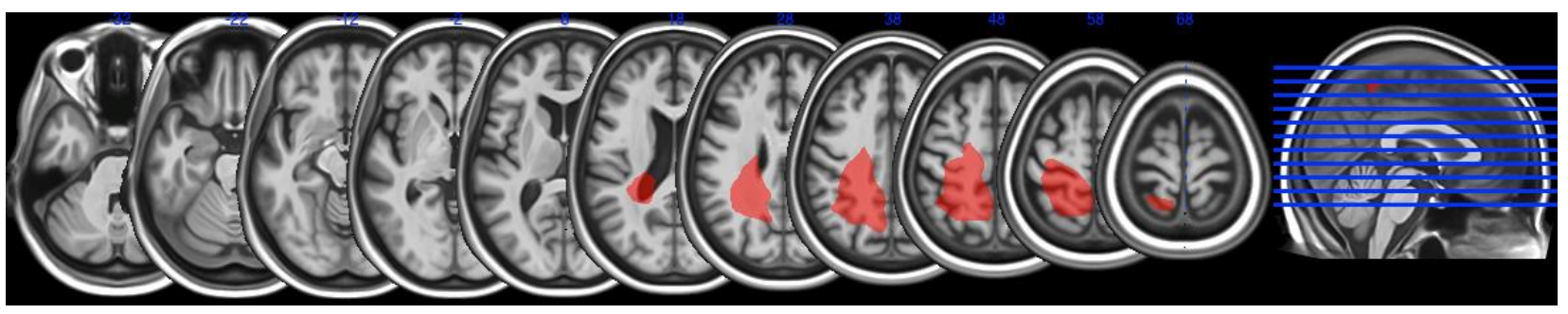

Figure A.8. MRI scan of patient CG

\section{EMH}

EMH is a 75 -year-old female from a large rural town in the North Island. In

December 2012, EMH presented at her local hospital complaining of slurred speech, facial droop and headaches. A CT scan revealed a small ring-enhancing lesion in the left posterior/frontal cortex. An MRI scan demonstrated that lesion to be $2.3 \mathrm{~cm}$ by $2.1 \mathrm{~cm}$. EMH had a background of lung cancer that had been treated with chemotherapy and radiation. A CT of her chest, abdomen and pelvis showed radiation related pneumonitis (inflammation of lung tissue) with no obvious tumour. On the $12^{\text {th }}$ of December, 2012, EMH underwent left frontal craniotomy and complete resection of sub central gyrus metastasis. Unfortunately, EMH was discharged from Wellington Hospital before postoperative testing could commence.

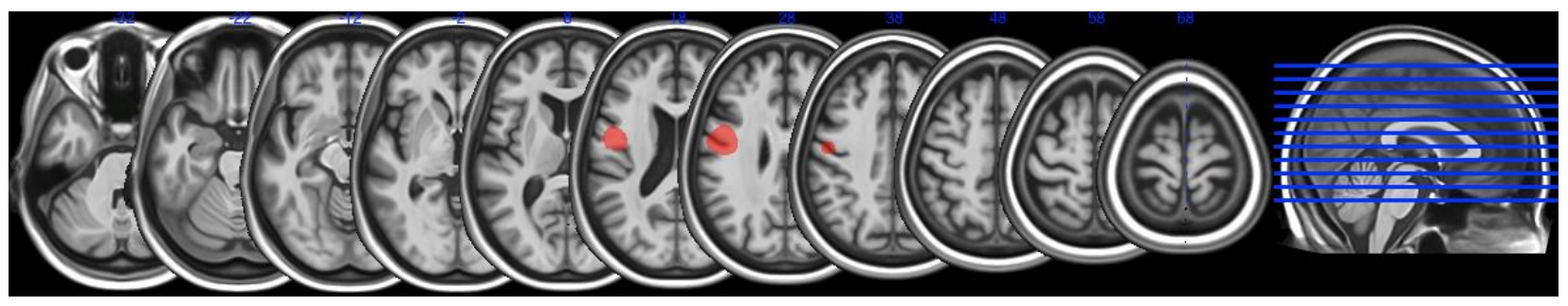

Figure A.9. MRI scan of patient EMH

EA

EA is a 70-year-old female from suburban Wellington. In January 2013 she was admitted to the Neurosurgery Department of Wellington Hospital with an incidentally 
discovered left frontal convexity meningioma. An MRI revealed that the mass measured approximately $3 \mathrm{~cm}$ in maximum AP diameter by $2.6 \mathrm{~cm}$ transversely. There was a small dural tail and slight mass effect on the anterior falx. EA underwent craniotomy in January 2013.

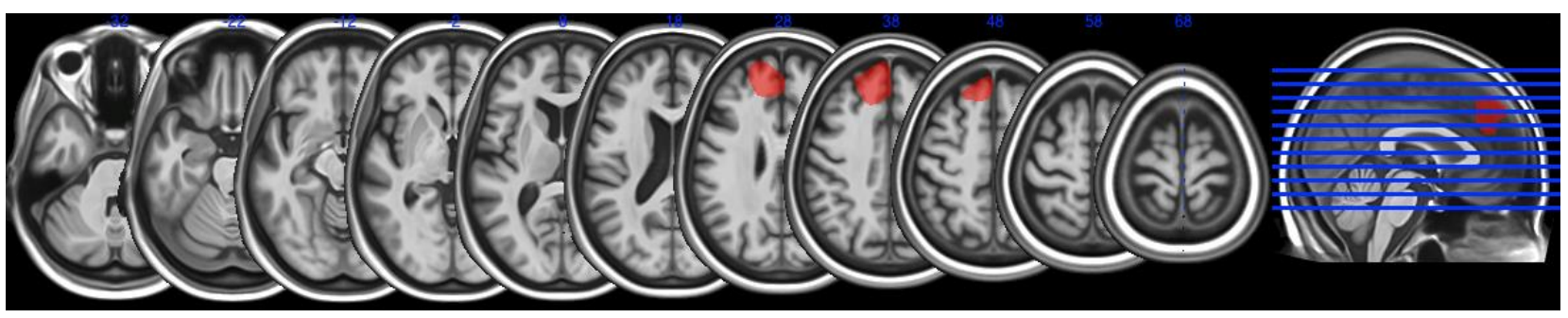

Figure A.10. MRI scan of patient EA

CA

CA is a 47-year-old female from a suburban town in the North Island. In February 2013 she presented at her local hospital with symptoms of headache and deteriorating cognitive function. Imaging demonstrated a large left sphenoid wing meningioma causing considerable midline shift and extensive central oedema. She was commenced on Dexamethasone, and improved rapidly. CA underwent craniotomy and tumour resection in early March 2013.

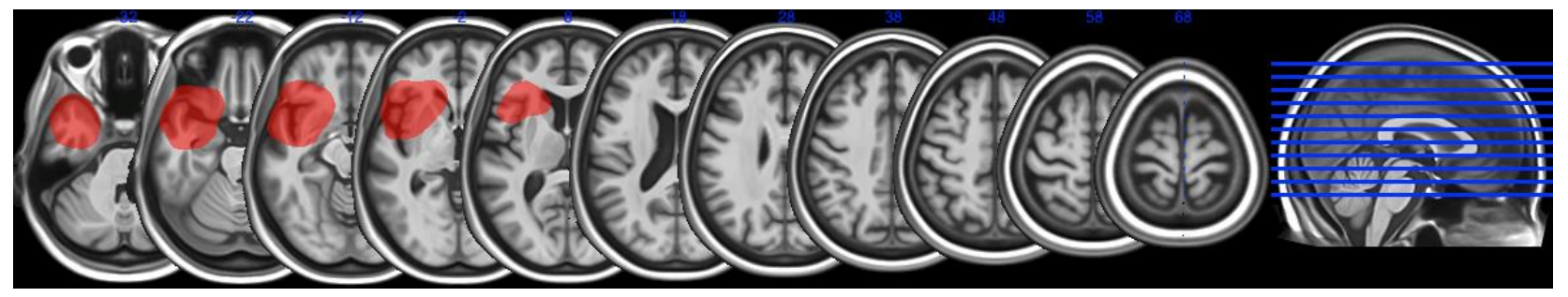

Figure A.11. MRI scan of patient CA

JM

$\mathrm{JM}$ is a 71-year-old female from a small rural town in the North Island. She presented in the Neurosurgical Ward in February with a three-to-four-week history of difficulty finding words, as well as poor comprehension. The dysphasia was resolved with Dexamethasone. She had no other focal neurological deficit and had no headaches. A CT and subsequent MRI report confirmed an irregular ring-enhancing left inferior frontal lesion and surrounding 
oedema. The appearance was consistent with a metastatic disease. JM also had a functional MRI to show the area of motor speech. This was due to the fact that the lesion was in eloquent brain and there was a moderate risk of postoperative speech disturbance. JM underwent craniotomy and exploration in March 2013.

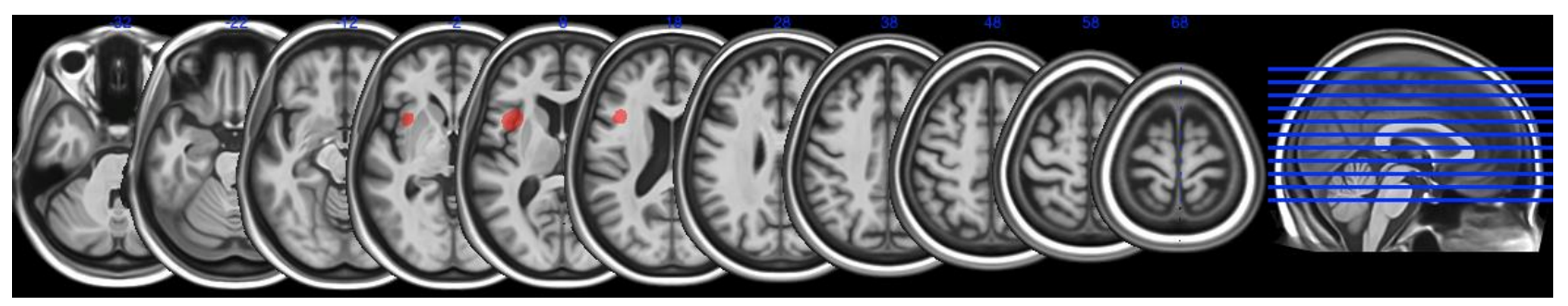

Figure A.12. MRI scan of patient JM

CR

CR is a 75-year-old female from suburban Wellington. She presented with difficulty writing and holding objects due to weakness of her right hand. She has intermittent episodes where there was tightness under the chin and a stiff mouth, which sometimes lasted for 10 minutes. There was no loss of awareness or loss of consciousness. She mostly had trouble writing and using the keyboard and mouse on a computer. There had been occasional dribbling on the right side. There had been no disturbance of speech, expression or compression. These symptoms improved once commencing Levetiracetam. An MRI scan in April showed a $4.5 \mathrm{~cm}$ left posterior frontal tumour with homogenous enhancement suggestive of a meningioma. Craniotomy occurred in May 2013.

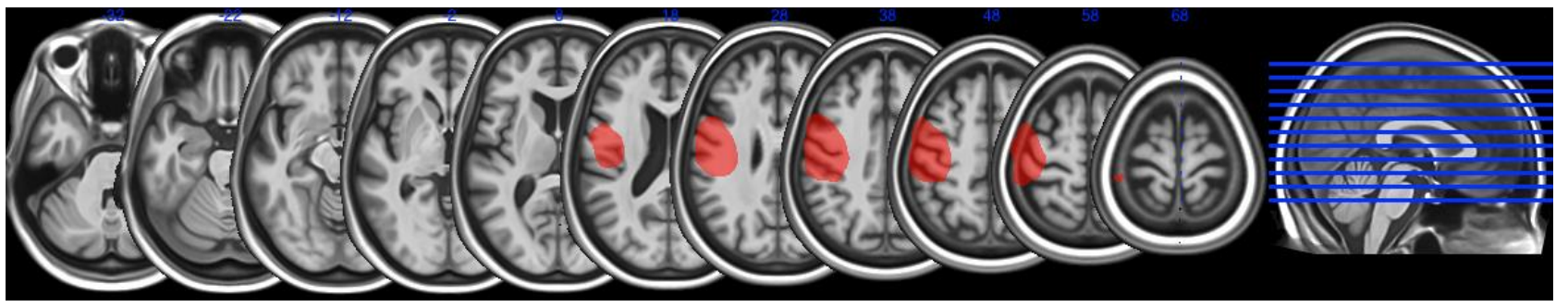

Figure A.13. MRI scan of patient CR 


\section{LC}

LC is a 43-year-old female from central Wellington. She presented with radicular pain, which had been presented over the last few months. On admission into the Neurosurgical Ward in September 2013, she remained asymptomatic expect for occasional headaches. Her neurological examination was normal expect for reduced sensation on her right foot. She was still experiencing radicular pain in her right leg. An MRI scan on admission revealed a lowgrade astrocytoma in the left posterior frontal lobe. Awake craniotomy was performed for resection of the tumour.

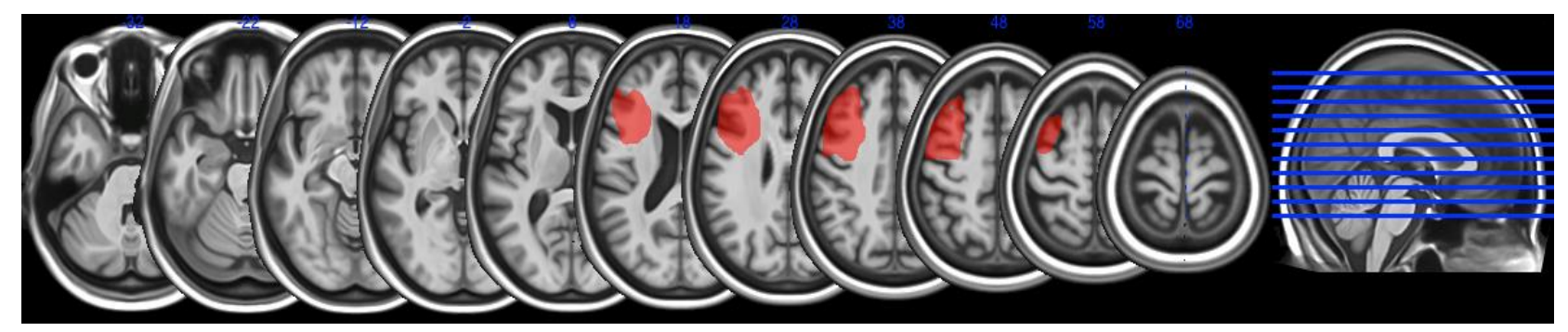

Figure A.14. MRI scan of patient LC

\section{DAP}

DAP is a 45-year-old male from a small rural town in the North Island. He presented at his local hospital a month before surgical admission due to a single epileptic fit. He also reported progressive deterioration in right leg function and headaches. An MRI revealed a tumour in the left parietal lobe adjacent to the motor strip. This had the characteristics of a low-grade glioma in the process of transforming to a higher-grade tumour. Craniotomy and resection occurred in September 2013. DAP had a seizure postoperatively, and consequently postoperative testing was not conducted. 


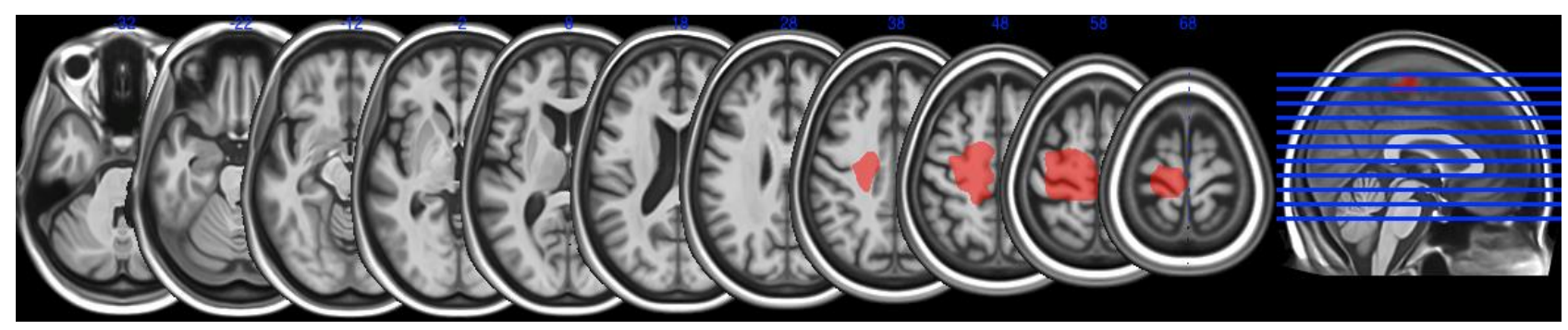

Figure A.15. MRI scan of patient DAP

MR

MR is a 68-year-old female from a small rural town in the North Island.

Approximately 12 years ago she underwent resection of a cutaneous malignant melanoma.

She had remained well since. However, in the weeks prior to her hospital admission, she had developed a profound affective disorder with very low mood. A CT scan was performed, demonstrating a left frontal and right thalamic enhancing lesion (in the right basal ganglia and marked compresses in the right frontal horn). Craniotomy occurred for resection of the left frontal lesion in September 2013. MR declined to participate in postoperative testing.

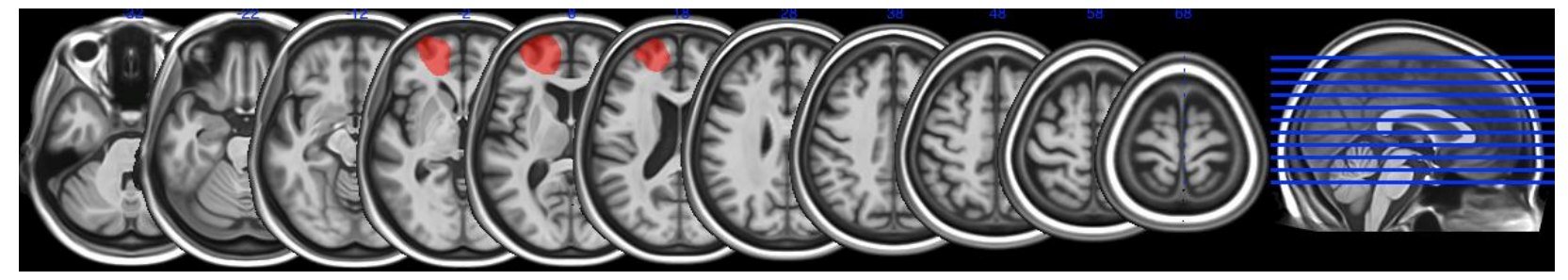

Figure A.16. MRI Scan of patient MR

TD

TD is a 30-year-old male from central Wellington. He was referred with a threemonth history of right-sided focal seizures. An MRI scan revealed a large generally nonenhancing mass in the left frontal region extending across the corpus callosum. The appearance was suggestive of an oligdendroglioma. Craniotomy occurred for debulking of the tumour in September 2013. 


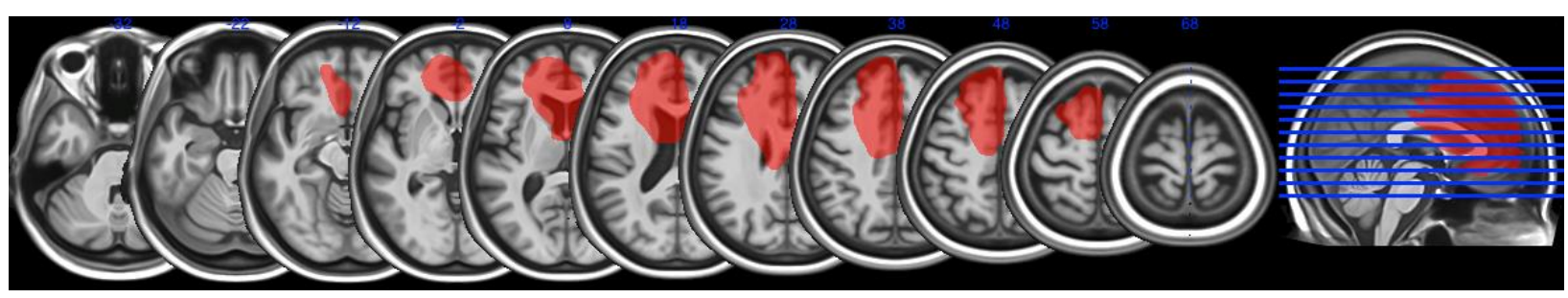

Figure A.17. MRI scan of patient TD

TF

$\mathrm{TF}$ is a 40-year-old female from a small rural town in the North Island. She presented with a gradual decline over the last few months with worsening headaches and personality change. On further questioning to her sister-in-law, they felt that her decline may have been going on for the last two years or so. She had had a strange sensation in her right leg, which she was unable to describe. Her behaviour became quite erratic, to a degree where she was admitted to hospital. She also had a few episodes of falls. Her CT and subsequent MRI scan showed a left frontal tumour. It was suggested that this tumour could either be a lymphoma or glioma. Craniotomy occurred for debulking in October 2013.

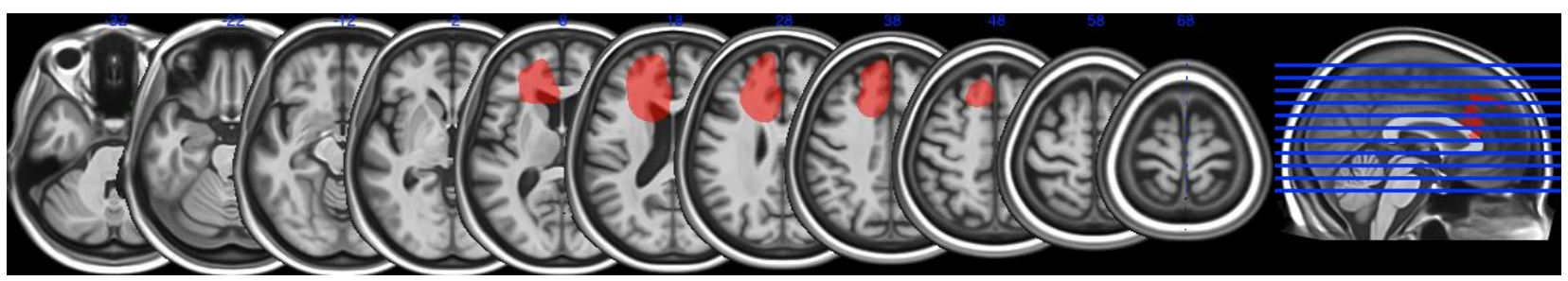

Figure A.18. MRI scan of patient TF

\section{Left Posterior Group}

AVG

AVG is a 42-year-old female from suburban Wellington. On the $21^{\text {st }}$ of March she presented with sudden deterioration in her right hand function and a degree of dysphasia. AVG also reported some difficulties with word finding and hand coordination over recent months, but was not $100 \%$ certain of this. Examination by Mr Andrew Parker revealed that she was fully conscious and oriented. Her cranial nerves appeared normal, she had no pronator drift, her speech has fluent and her gait was normal. A CT and MRI scan confirmed 
the presence of a large durally based mass in the left convexity. Bony changes surrounding the lesion were suggestive of a meningioma. Further investigation into AVG's acute dysphasia by the primary investigator revealed that she experienced sudden reading difficulties. During her interview with the primary investigator, she complained of reading difficulties; she explained that she could see individual letters within a word but could not decipher the overall word. This was relieved with Dexamethasone. Surgery was performed ten days later to remove the bulk of the tumour.

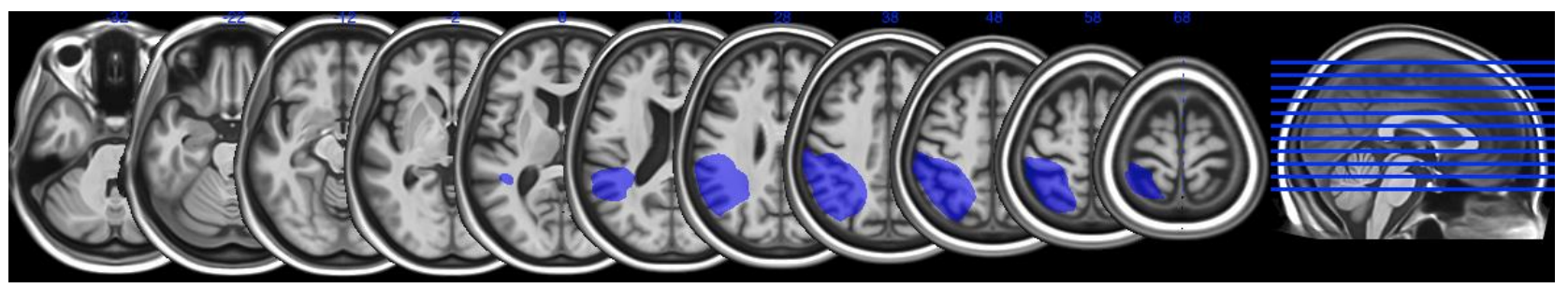

Figure A.19. MRI scan of patient AVG

\section{BCA}

BCA is a 56-year-old male from Wellington. BCA presented at the Neurosurgery Clinic at Wellington Hospital in April 2012. BCA underwent debulking of a left occipital glioblastoma in April 2011, which was followed by radiation. He remained relatively well until early 2012, when he complained of increasing forgetfulness, difficulties with reading and intermittent episodes of dysphasia. He also reported recent headaches, which became worse after lying down for a nap. There was no nausea, vomiting or seizure. BCA was seen by Mr Andrew Parker in February 2012. He presented with a right homonymous hemianopia, probably dating from the time of his surgery, but the acuity in his reaming visual fields had remained intact. An MRI scan performed at that time revealed a recurrence of the glioblastoma around the site of his previous surgery with oedema extending into the left temporal lobe. Surgery was performed in April 2012, in order to attempt further debulking. 


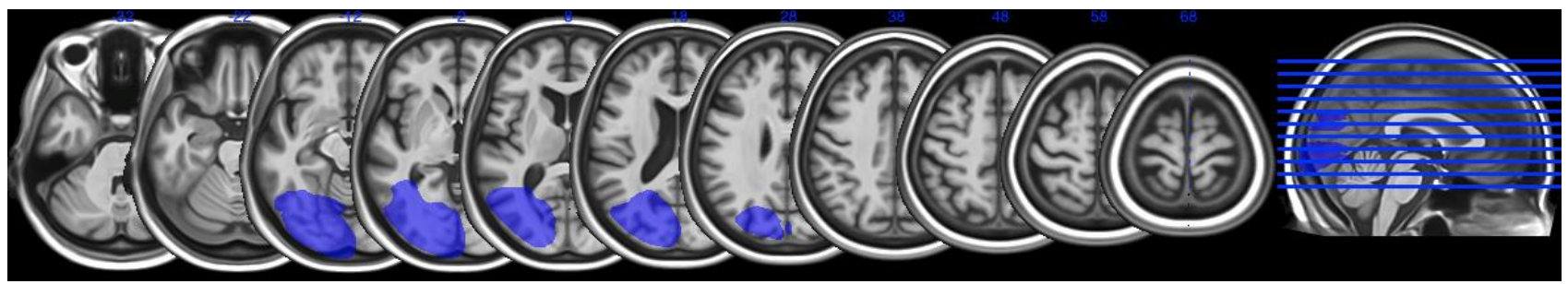

Figure A.20. MRI scan of patient BCA

BD

BD is a 62-year-old female from a small rural town in the North Island. She presented at the Neurosurgery Ward in March 2013. Here she described symptoms of discomfort in the proximal right lower limb. These had been present for over a year, and she increasingly seemed to drag this leg. She had longstanding numbness over the lateral aspect of the left calf. Her balance had been subtly affected. She also described a "full feeling" in her head that had been present since at least September 2012. She also reported intermittent numbness over the left side of her face and occasional headaches. Her husband reported some subtle changes in her mood, that she was sleeping far more than she used to, and subtle lapses in memory. On examination, cranial nerves where normal with the exception of early papilledema in the left eye. She exhibited normal tone, power and co-ordination of all her limbs. Her CT scan demonstrated a $3 \times 4 \mathrm{~cm}$ meningioma arising from the superior sagittal sinus. Craniotomy and resection occurred in March 2013. Unfortunately, BD’s MRI scan was unavailable.

AM

AM is a 38-year-old female from Wellington. In May 2012, AM presented at Wellington Hospital complaining of a sudden inability to read and spell certain words, and word finding difficulties. She also exhibited right lower facial weakness. A CT scan revealed an irregular mass lesion measuring up to $33 \mathrm{~mm}$ in diameter in the posterior left parietal lobe, suggestive of a glioblastoma. Surgery was performed in late May 2012. 


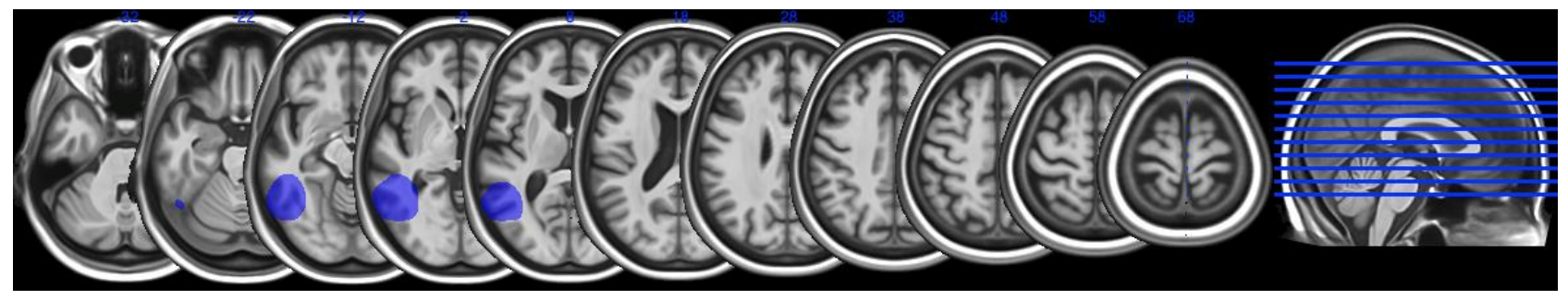

Figure A.21. MRI scan of patient AM

\section{RJ}

$\mathrm{RJ}$ is a 64-year-old female from a small rural town in the North Island. She presented with a fall and right leg weakness. She experienced a four-to-five-week history of episodic twitching in her arms. A CT scan showed a left parietal lobe tumour (most likely metastasis from a mass in her right lung). Upon admission to the Neurosurgical Ward, she declined surgery, and therefore no postoperative testing was conducted.

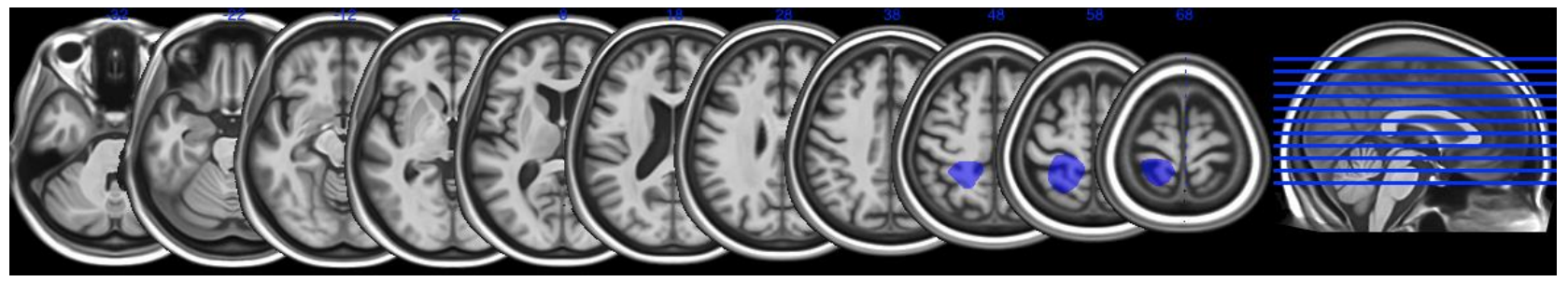

Figure A.22. MRI scan of patient RJ

\section{Right Frontal Group}

\section{LA}

LA is a 63-year-old female from a rural town in the North Island. Due to the fact that all of LA's consultations occurred in her hometown, there was no medical history available at Wellington Hospital. However, her neurologist described one week of progressive left arm weakness that resolved with steroids. A CT and MRI scan performed at Wellington Hospital in April 2012 revealed a right frontal tumour, suggestive of a glioblastoma. Craniotomy was performed the following day. 


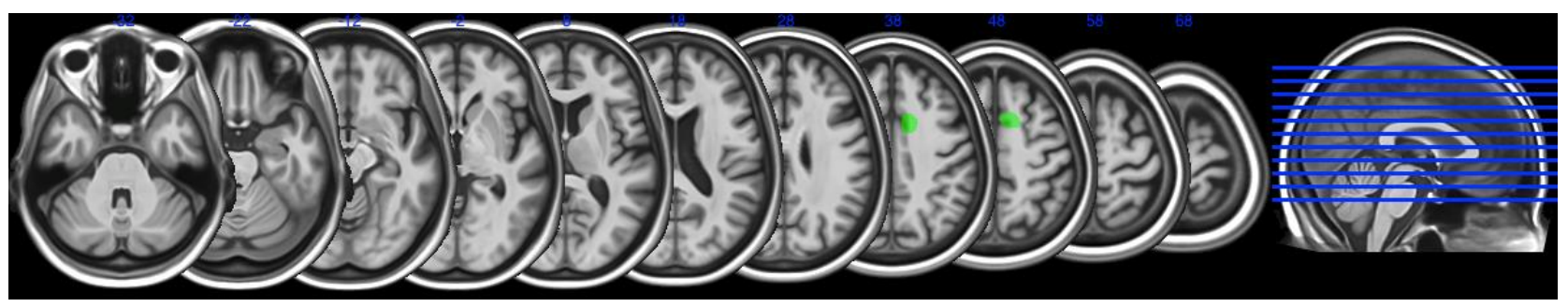

Figure A.23. MRI scan of patient LA

\section{CM}

CM is a 57-year-old male from Wellington. When seen in March 2011, CM described a possible seizure, which occurred the previous March whilst he was cycling around the bays. He was picked up by an ambulance. He was apparently seen by the security monitors near the tunnel at the sound end of the airport, and seen to wobble and fall to the ground. CM also reported an episode of sudden severe pain in his head about a year and a half ago, lasting 60 seconds. Recently he had also experienced two episodes of hot flushes. A CT performed at that time, and an MRI performed the following May, showed an ill-defined enhancing mass within the anterior right frontal lobe in a parafalcine location. Surrounding the mass there was a modest degree of vasogenic oedema within the right frontal lobe, which extended towards the corpus callosum but did not cross through into the left hemisphere. This presentation suggested a primary tumour, most likely an oligodendroglioma. Surgery was performed in June 2012.

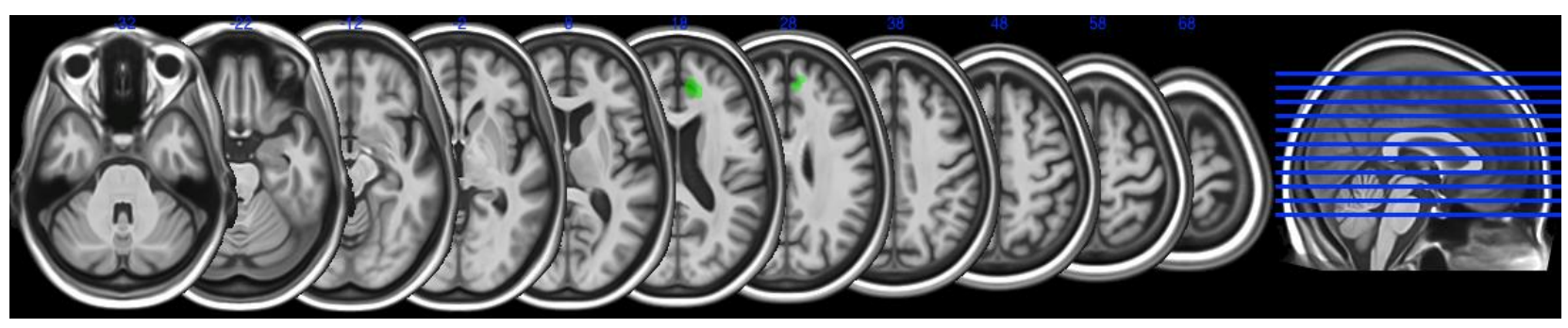

Figure A.24. MRI scan of patient CM 


\section{LW}

LW is a 66-year-old female from Hastings. Since October 2011 she has suffered from right-sided headache, which was initially severe but had reduces to manageable levels. In July 2012, LW was examined by Mr Parker. On examination, she presented as alert and orientated. She was slightly short of breath on minimal exertion. No focal neurological defects were detected. An MRI performed in June 2012 showed an extra axial durally based lesion in the right posterior frontal convexity region. It had a well-defined border but a somewhat lobulated appearance. There was a small amount of associated oedema. Diagnostic possibilities included meningioma or possible dural metastasis. Excision of the tumour was performed in August 2012.

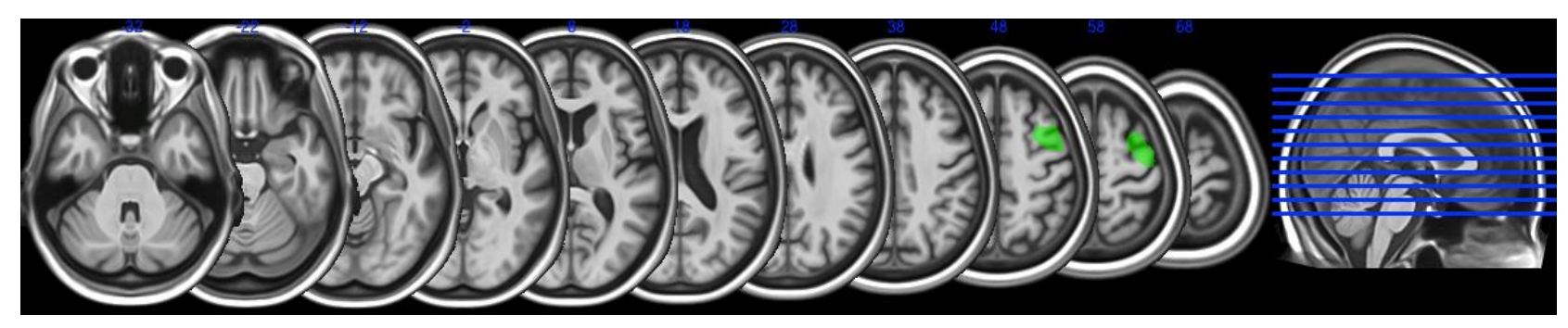

Figure A.25. MRI Scan of patient LW

KB

$\mathrm{KB}$ is a 30 -year-old female from a suburban town in the North Island. KB presented at her local hospital with a seven-month history of headache and associated periorbital pain and slight proptosis. This was associated with blurred vision and diplopia in particular on upward gaze. CT and MRI reports confirmed a sphenoid wing meningioma on the right side with mass effect on the hemisphere and midline shift. There was an associated hyperostosis of the roof and lateral wall of the right orbit, causing mild proptosis. Craniotomy and tumour resection occurred on the $14^{\text {th }}$ of March. Unfortunately, post surgery KB had eyelid oedema, and she was only able to open her eyes for a short period of time. Consequently, postoperative testing was cancelled. The MRI scan for patient KB was unavailable. 
ES

ES is a 78-year-old male from a small rural town in the North Island. He presented with a two-week history of progressively worsening confusion and unsteadiness of gait. A CT and MRI scan of his brain demonstrated a right frontal heterogeneously enhancing lesion on the periphery with central hypointense region suggestive of neurosis with focal and generalised mass effect. Craniotomy for resection occurred in December 2012.

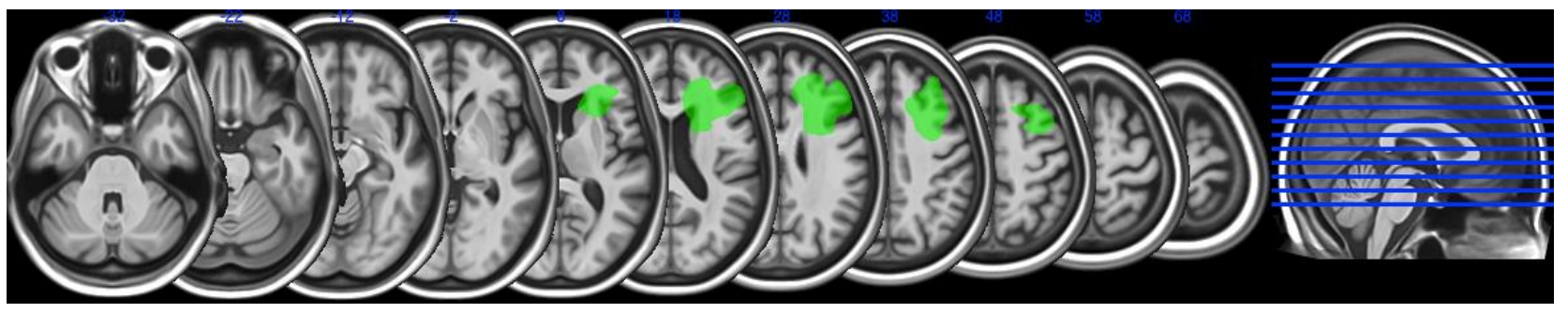

Figure A.25. MRI scan of patient ES

TT

TT is a 36-year-old female from suburban Wellington. She presented with slow recurrence of her posterior frontal diffuse astrocytoma (grade 2). This was previously resected in September 2006. She had been under surveillance with slow increase in the bulk in the right posterior frontal region. There had been an increase in seizures over the past six months. In particular over the last two months she has had seizures up to once a week. An MRI scan in February 2013 showed the increase in bulk of the tumour with a hint of enhancement, which was confirmed on the stealth MRI. Craniotomy and macroscopic resection occurred in May 2013.

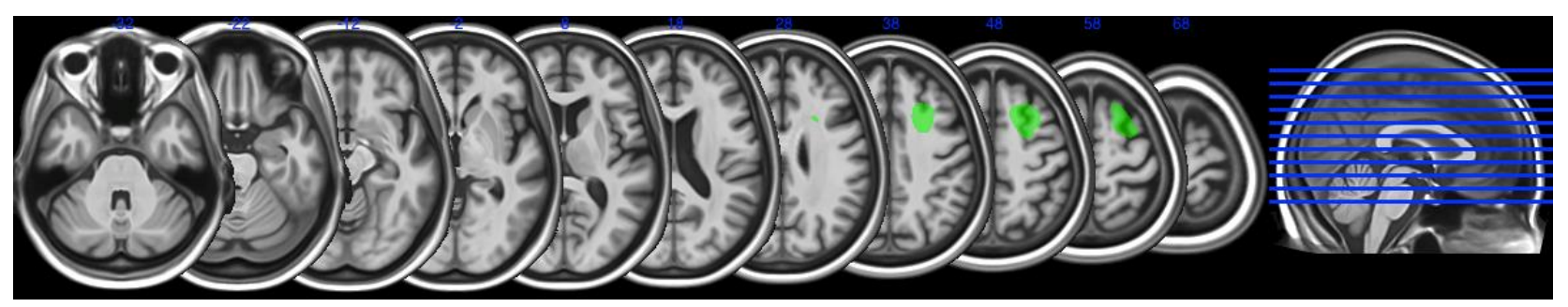

Figure A.26. MRI scan of patient TT 
SMC

SMC is a 63-year-old male from a small rural town in the South Island. He presented with personality changes and impaired memory over the past few weeks. Investigations with MRI showed a large right frontal cystic mass with rim enhancement causing mass effect consistent with a cystic glioblastoma. Craniotomy and tumour removal occurred in September 2013.

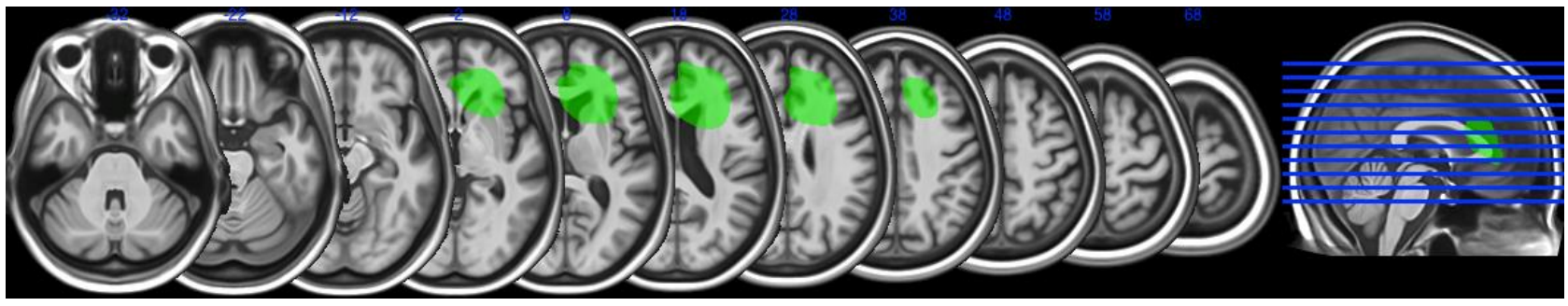

Figure A.27. MRI scan of patient SMC

DF

DF is a 40-year-old male from central Wellington. He had a history of several focal seizures where he had sat up shaking, but there was no loss of consciousness. An MRI in May 2013 revealed a low-grade astrocytoma in the opercular, frontal and temporal region extending into the insular cortex, just shy of the external capsule. Craniotomy occurred for debulking in November 2013.

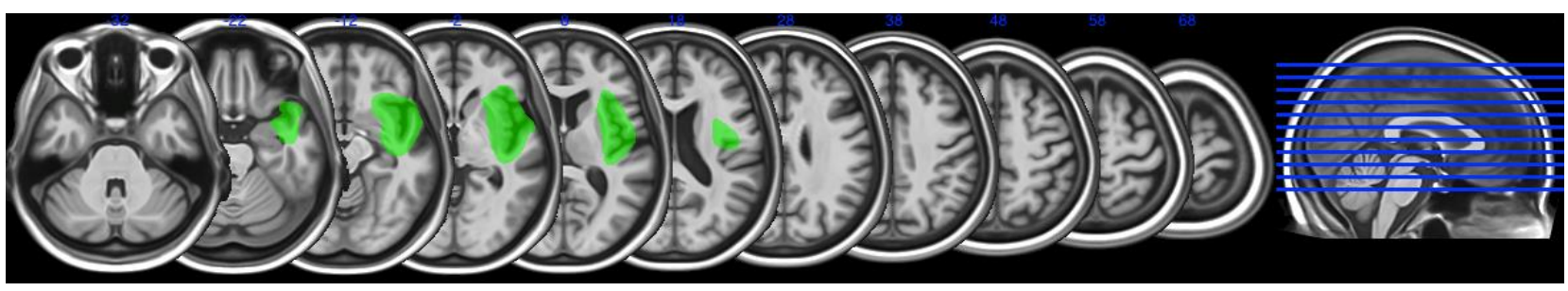

Figure A.28. MRI scan of patient DF

GP

GP is a 73-year-old male from a small rural town in the North Island. Eighteen years ago he underwent resection of a right frontal meningioma. He presented in November 2013 with a several-month history of lethargy, disturbed gait and impaired coordination. His 
imaging demonstrated a large right frontal tumour with considerable oedema and associated midline shift. Appearances were consistent with a large recurrent meningioma. Craniotomy occurred one week later for resection of the meningioma.

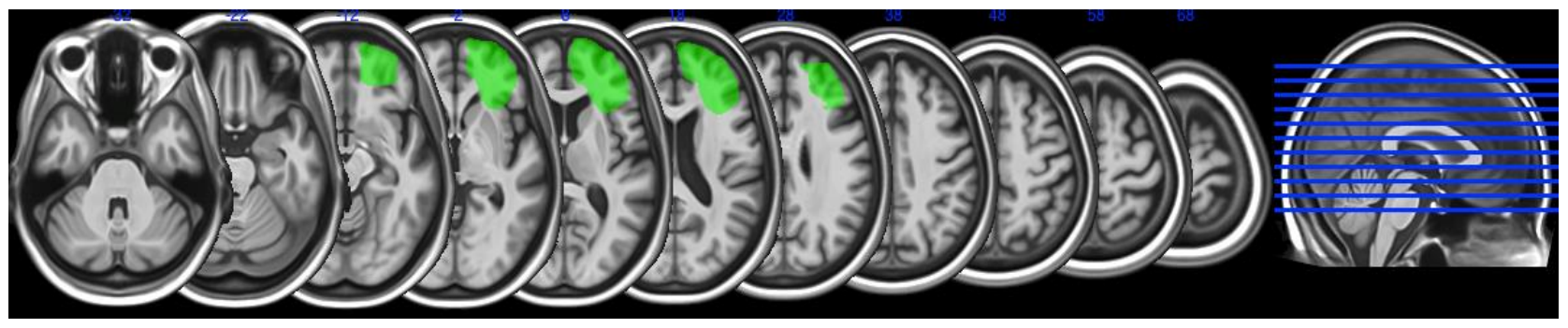

Figure A.29. MRI scan of patient GP

\section{MRO}

MR is a 60-year-old male from a small rural town in the north of the South Island. He had a two-year history of weakness of his left foot and increased difficulty walking over the last six months with foot drop, and associated with headaches. CT and MRI scan confirmed a large frontoparietal convexity parasagittal tumour with homogeneous enhancement consistent with probable meningioma. There was a mass effect on the right lateral ventricle with midline shift. Craniotomy for removal of the meningioma occurred in September 2013.

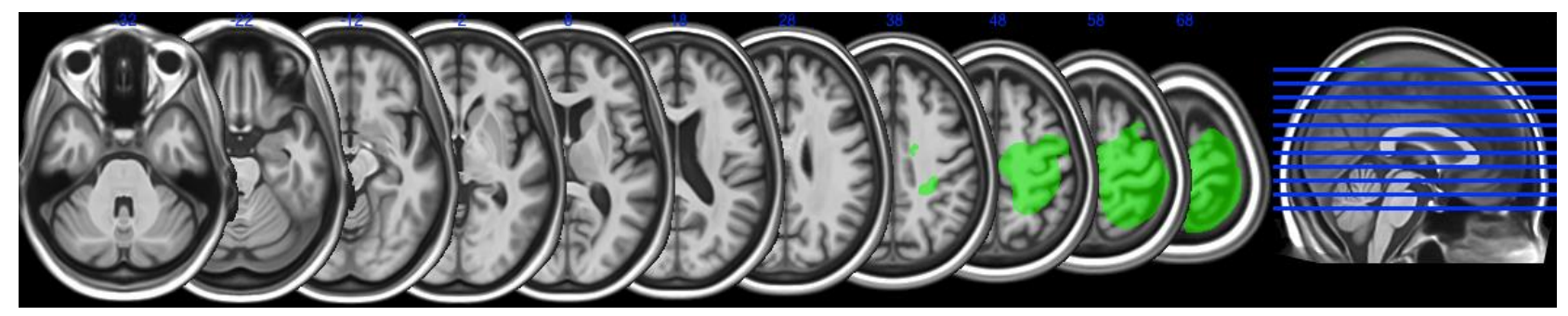

Figure A.30. MRI scan of patient MRO

\section{Right Posterior Group}

RG

RG is a 56-year-old male from a rural town in the North Island. In March 2011, RG underwent surgery for debulking of a right gliosarcoma in his right occipital lobe. This was further treated by radiation therapy, which was completed in early June 2011, and adjuvant 
Temozolomide for six cycles, which was completed in November 2011. RG had an MRI scan in January 2012. In addition to the expected postsurgical changes, it showed there was evidence that the tumour had not been completely removed. Surgery was performed to debulk the tumour in June 2012.

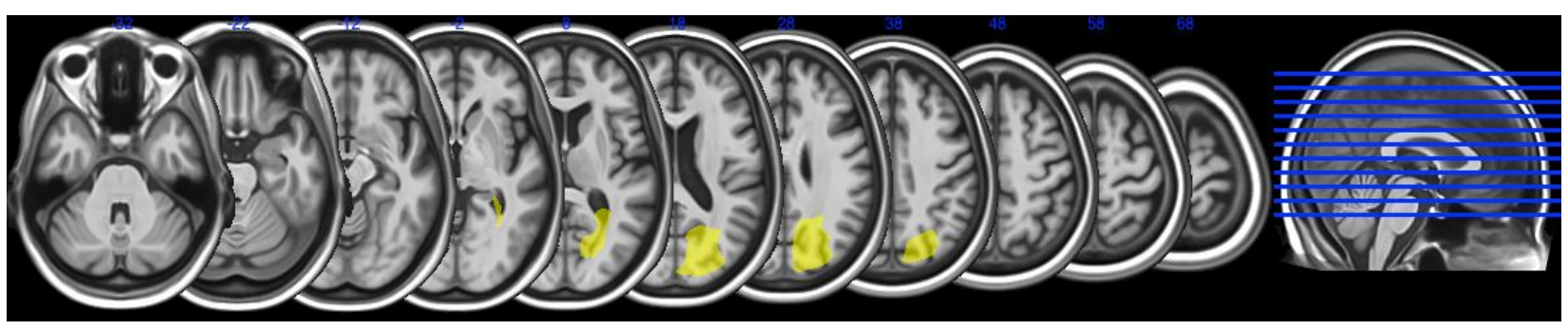

Figure A.31. MRI scan of patient RG

\section{TKH}

TKH is a 60-year-old male from a large town in the North Island. He was seen in August 2012. He had complained of severe headaches over the past few weeks. Neurological examination revealed no major neurological deficit, although there was some suggestion that his vision may have deteriorated subtly. An MRI revealed a tumour in the right temporal lobe consistent with glioblastoma. Surgery was conducted in late August 2012.

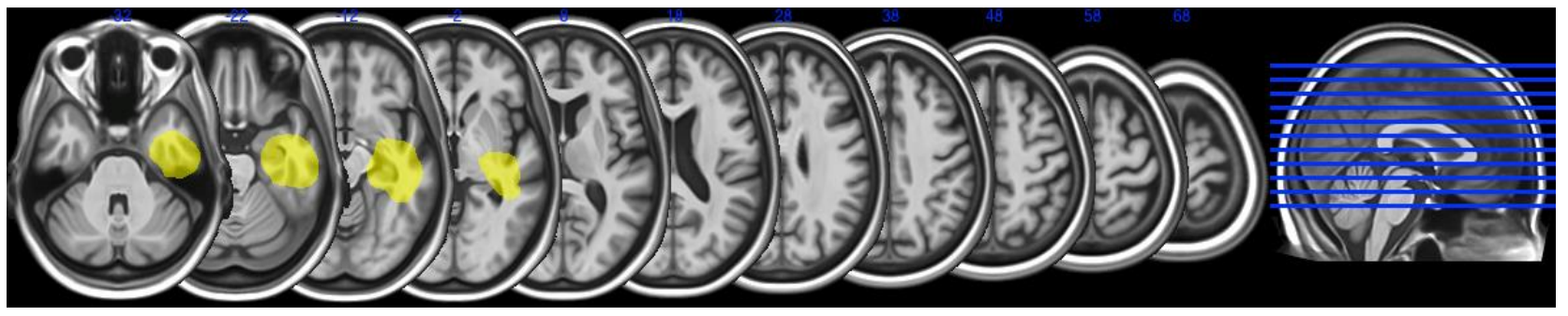

Figure A.32. MRI scan of patient RG

\section{JAS}

JAS is a 55-year-old male from a large rural town in the North Island. In September 2012, at his local hospital, he complained of headaches to vertex lasting 30 minutes, which had increased over the past week in severity and frequency, and now included nausea and visual disturbances lasting 15 minutes. A radiologist revealed that JAS had a right temporal 
tumour with mass effect on the temporal horn, lateral ventricle and midline, as well as on the brain stem which is compressed to the left. Surgery occurred on the $20^{\text {th }}$ of September.

Unfortunately, JAS was discharged from Wellington Hospital before postoperative assessment could occur.

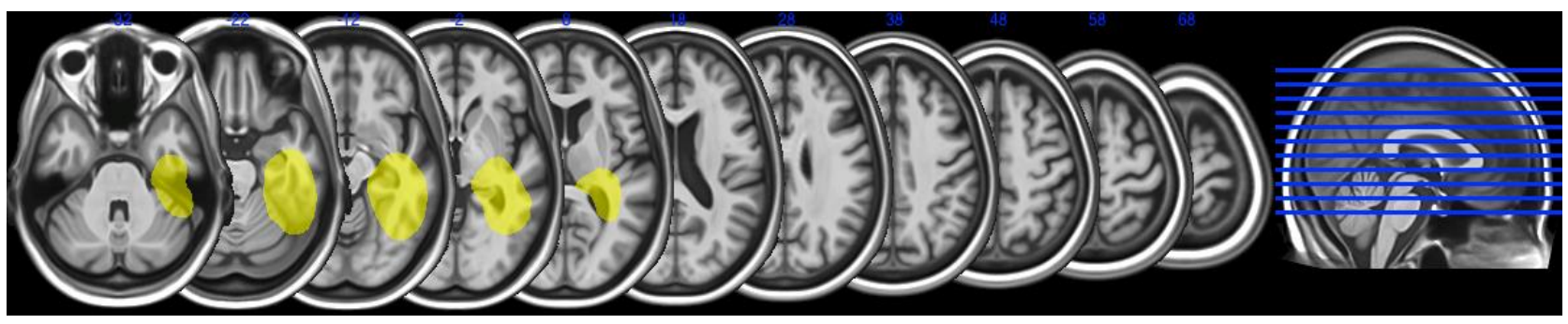

Figure A.33. MRI scan of patient JAS

AEK

AEK is a 52-year-old female from central Wellington. She presented at Wellington Hospital with left facial drop. She had minimal headaches but complained of lethargy for the last few months. A CT and subsequent MRI revealed a right posterior temporal tumour suggestive of malignant glioma. AEK underwent craniotomy and resection of the right fusiform gyrus malignant glioma in March 2013.

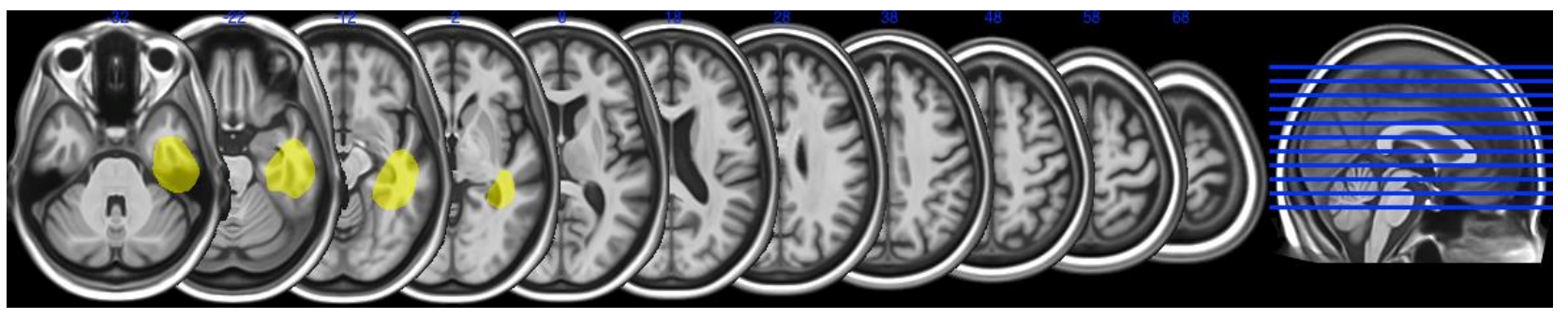

Figure A.34. MRI scan of patient AEK

JB

JB is a 47-year-old female from a large rural town in the North Island. JB was referred from Masterton Medical centre, and consequently there was no medical records. Personal correspondence with neurosurgeon Mr Andrew Parker revealed that JB had a right temporal epidermoid lesion, and this was confirmed with her MRI scan. Her surgery was conducted in May 2012. 


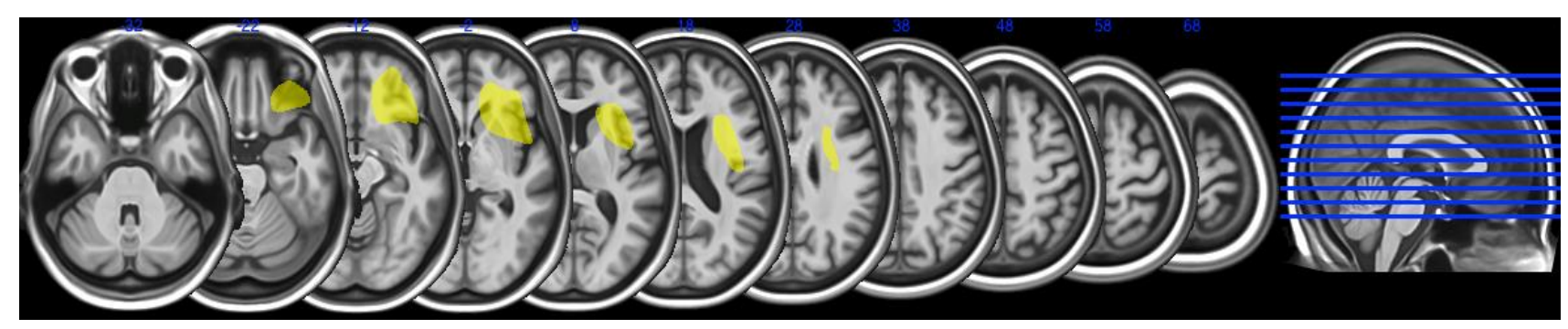

Figure A.35 MRI scan of patient JB

\section{Multiple Lesions}

\section{PAJ}

PAJ is a 65-year-old male from Wellington. In early July he complained of wordfinding difficulties extending back around two months, and facial spasms accompanied by slurred speech and left facial weakness, which occurred more recently. These facial spasms were suggestive of seizures. An MRI scan of PAJ's head showed a right posterior inferior frontal tumour in the area of the pars opercularis/subcentral gyrus (histology confirmed a glioblastoma) and a posterior left temporal ring-enhancing lesion. Two surgeries were performed in July 2012: one to resect the frontal lesion, and another one week later to resect the left temporal tumour. Preoperative and postoperative testing was conducted for the first surgery only.

$\mathbf{A E}$

$\mathrm{AE}$ is a 46-year-old man from central Wellington. He presented with complex partial seizures. An EG showed focus at the left temporal region. He was known to have multiple intracranial cavernous haemangiomas. There was also a right posterior frontal meningioma. A left posterofrontal lesion had increased in size, and was therefore removed by craniotomy in April 2013. 
Appendix B: Information Sheet for Healthy Controls

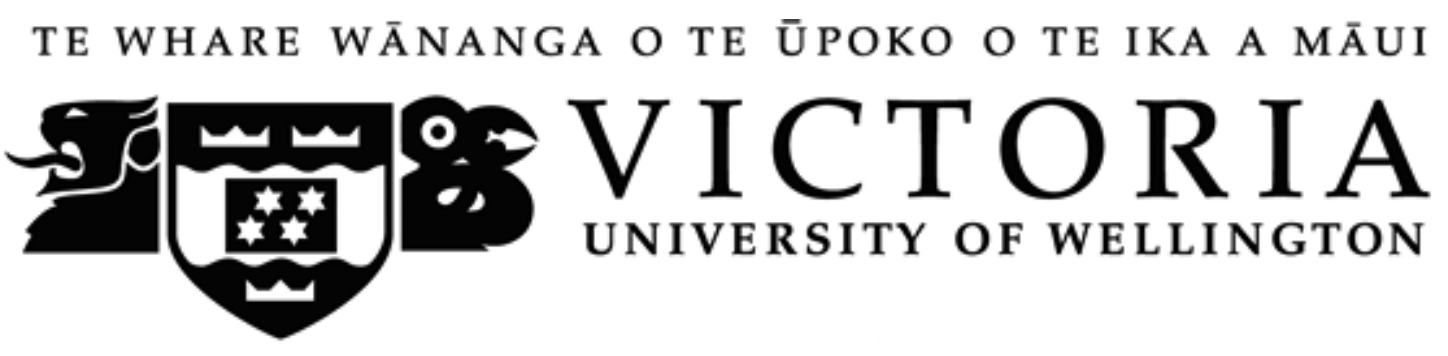

Information Sheet

Study: A New Test Battery for Examining Language in Individuals Undergoing
Neurosurgery

Josh Faulkner

PhD Candidate

joshua.faulkner@vuw.ac.nz

0274240624
Dr Carolyn Wilshire, PhD

Supervisor

Carolyn.wilshire@vuw.ac.nz

(04)463 6036

\section{What is the purpose of this research?}

- This research will allow us to assess the effectiveness of different kinds of language tasks for assessing language before, and after neurosurgery for the removal of a brain tumour. The results obtained may help clinicians to assess language more effectively in this context. In order to determine the effectiveness of these tests we also need to examine test performance in individuals with no neurological impairments. These results can then be compared with individuals undergoing neurosurgery to determine the extent of their possible language deficits.

\section{Who is conducting the research?}

- We are researchers in the School of Psychology at Victoria University of Wellington. Dr Wilshire is supervising the project. This research has been approved by the School of Psychology Human Ethics Committee under delegated authority of Victoria University of Wellington's Human Ethics Committee.

\section{What is involved if you agree to participate?}

- If you agree to participate in this study you will partake in one testing sessions. During each session, you'll be asked to do a range of simple language tasks that aim to test a range of language abilities.

- The language tests will involve you naming pictures, giving a list of words that start with a particular letter of the alphabet and category, repeating words presented by the examiner, giving an action word that is associated with an object, determining if a heard word matches that of a picture and naming the colour of written words.

- We anticipate that your total involvement will take no more than one hour.

- During the research, you are free to withdraw at any point before your data has been collected. You may participate in any or all testing phases: it's up to you. 


\section{Privacy and Confidentiality}

- We will keep your consent forms and data until the research project has been completed and the findings are published.

- You will never be identified in this research project or in any other presentation or publication. The information you provide will be coded by number or initials.

- In accordance with the requirements of some scientific journals and organizations, your coded data may be shared with other competent researchers.

- Your coded data may be used in other, related studies.

- A copy of the coded data will remain in the custody of Dr Wilshire.

\section{What happens to the information that you provide?}

- The data you provide may be used for one or more of the following purposes:

- The overall findings may be submitted for publication in a scientific journal, or presented at scientific conferences.

- The overall findings will form part of a PhD thesis, which, will be submitted for assessment.

If you would like to know the results of this study, they will be available approximately in December 2012 from the following sources:

- Information posted/emailed to you upon request

If you have any further questions regarding this study please contact any one of us above. 


\section{Statement of Consent}

I have read the information about this research and any questions I wanted to ask have been answered to my satisfaction.

I agree to participate in this research. I understand that I can withdraw my consent at any time, prior to the end of my participation.

Name:

Signature:

Date:

I would like to receive a copy of the results: Yes $\square$ No

Email:

Copy to:

[a] participant,

[b] researcher (initial both copies below) 


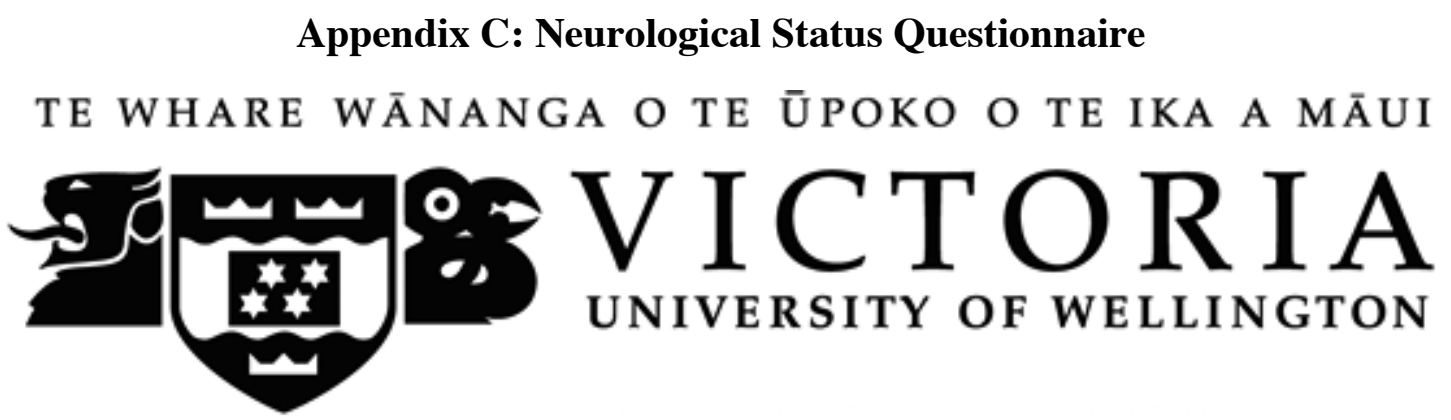

Neurological Status Questionnaire

Study: A New Test Battery for Examining Language in Individuals Undergoing Neurosurgery

\section{Principal Investigator: Josh Faulkner, School of Psychology}

It would help us with our research if you were able to provide the following additional information. Please note that completion of the following questions is entirely optional and confidential.
Age:
Sex: M/F
Handedness: L/R

Highest level of education obtained:

Have you ever suffered from any visual and/or hearing impairments? (please specify)

Have you ever experienced a neurological event such as a stroke, or other brain injury? (please specify) 
Appendix D: Information and Consent Form for Brain Tumour Patients

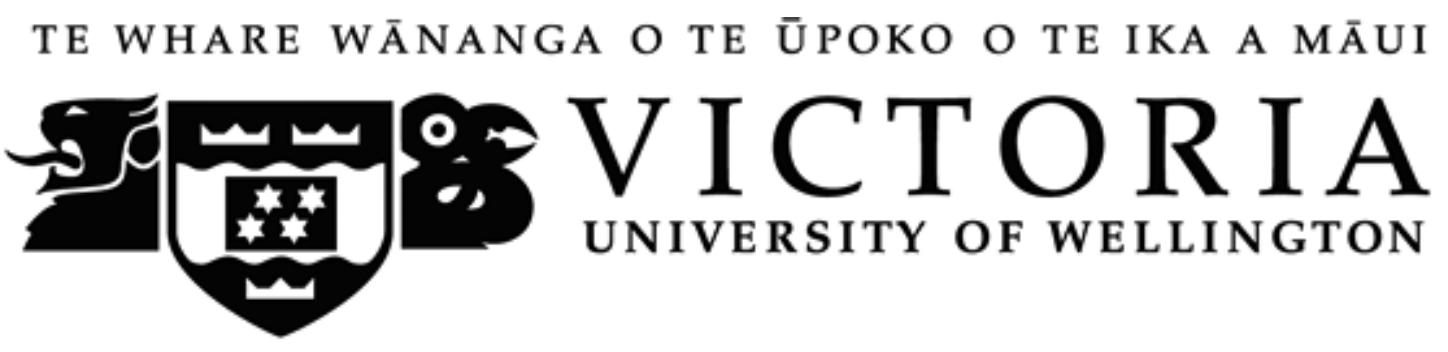

Information Sheet

Study: The assessment of language before, and after neurosurgery.

Josh Faulkner

PhD Candidate

joshua.faulkner@vuw.ac.nz

0274240624
Dr Carolyn Wilshire, PhD

Primary Supervisor

carolyn.wilshire@vuw.ac.nz

(04) 4636036
Kay Cunningham, MA, DipClinPsyc Clinical Supervisor

kay.cunningham@xtra.co.nz

You are invited to take part in a research project for Victoria University of Wellington.

Please take your time to read through the information sheet. Your participation is entirely voluntary (your choice). You do not have to take part in this study, and if you choose not to take part you will receive the standard treatment/care available. Participation in this study will be stopped should any harmful effect appear or if the doctor feels it is not in your best interest to continue

\section{What is the purpose of this research?}

- This research will allow us to assess the effectiveness of various different kinds of language tasks for assessing language before, and after neurosurgery for the removal of a brain tumor. The results obtained may help clinicians to assess language more effectively in this context. The information we gain may also provide us with useful insights into human language and the way it is organised in the brain.

\section{Who is selected for the study?}

- All patients undertaking neurosurgery for the removal of a brain tumor in Wellington hospital are invited to participate in this study

$\bullet$

Where will the study take place?

- Testing before and after will take place at the Neurology department in Wellington hospital. Participants may also be asked if they would be willing to participate in a follow-up visit at their homes 6-8 weeks following surgery.

What is involved if you agree to participate?

- If you agree to participate in this study you will partake in two testing sessions; before surgery, and after surgery. During each session, you'll be asked to do a range of simple language tasks that aim to test a range of language abilities. 
- The language tests will involve you naming pictures, giving a list of words that start with a particular letter of the alphabet and category, repeating words presented by the examiner, giving an action word that is associated with an object and reading coloured words whilst ignoring their colour.

- For the before- and after-surgery testing, we will visit you in your hospital ward at a time that suits you.

- We anticipate that your total involvement will take no more than one hour per session.

- During the research, you are free to withdraw at any point before your data has been collected. You may participate in any or all testing phases, it's up to you.

\section{Who is conducting the research?}

- Josh Faulkner is a PhD student at Victoria University and is undertaking this research as part of his doctorate thesis. Ms Cunningham, a registered Clinical Neuropsychologist may assist in administering language tests. Dr. Wilshire, a Senior Lecturer at the School of Psychology at Victoria University of Wellington is the primary supervisor for this project.

\section{Privacy and Confidentiality}

- We will keep your consent forms and data until the research project has been completed and the findings are published.

- You will never be identified in this research project or in any other presentation or publication. The information you provide will be coded by number or initials.

- In accordance with the requirements of some scientific journals and organizations, your coded data may be shared with other competent researchers.

- Your coded data may be used in other, related studies.

- A copy of the coded data will remain in the custody of Dr. Wilshire.

\section{What happens to the information that you provide?}

- The data you provide may be used for one or more of the following purposes:

- The overall findings may be submitted for publication in a scientific journal, or presented at scientific conferences.

- The overall findings will form part of $\mathrm{PhD}$ thesis, that will be submitted for assessment.

\section{What are the benefits of this study?}

- This study will allow participants to obtain detailed feedback about their specific strengths and weaknesses

- Given that the language evaluation will be more extensive than is normally given in these cases, the information we gain may be more useful for other health practitioners

\section{What are the risks of this study?}

- Participants may be experiencing fatigue and possible distress during testing before and after brain surgery. To minimize any potential harm, testing sessions are kept as short as possible and remember, you are free to stop at any time for any reason. 


\section{What are the inclusion and exclusion criteria for this study?}

- People invited to participate in this study will be those who:

- Have been admitted to Wellington Hospital for neurosurgery

- Are at least 18 years of age

- Whose surgery will focus on a key brain region involved in language

- Those not eligible are:

- Those whose native language is not English

- Anyone who, in the opinion of the individual's surgical team, may find the testing unduly stressful.

\section{Results}

- If you would like to know the results of this study, they will be available approximately in December 2015 from the following sources:

- Information posted/emailed to you upon request

\section{Statement of Approval}

- This study has received ethical approval from The New Zealand Health and Disability Ethics Committee, ethics reference number CEN/11/07/037

If you have any queries or concerns regarding your rights as a participant in this study, you may wish to contact an independent health and disability advocate:

Free phone: 0800555050

Free fax: 08002 SUPPORT (0800 2787 7678)

Email: advocacy@hdc.org.nz

If you have any further questions regarding this study please contact any one of us above. 


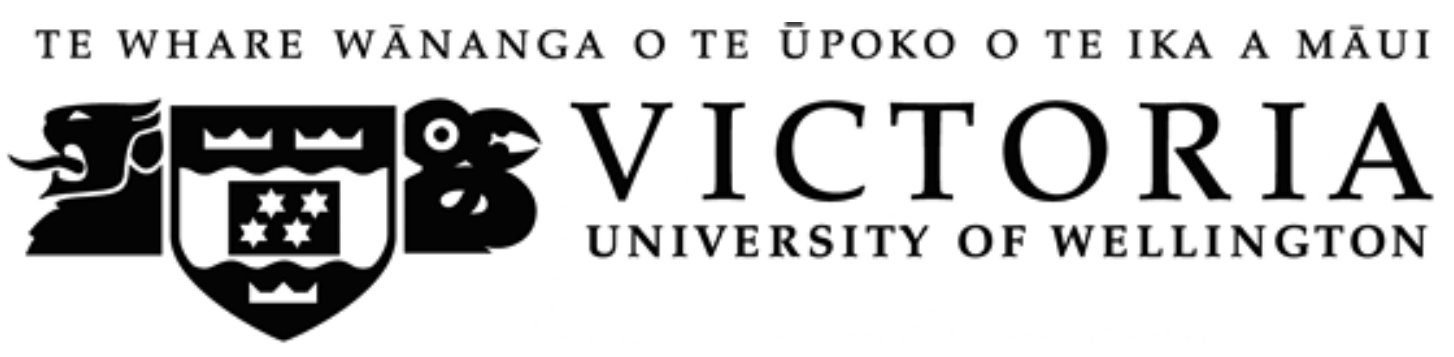

Consent Form

Study: The assessment of language before and after neurosurgery.

I have read and I understand the information sheeted dated for volunteers taking part in the study designed to test specific language functioning before and after neurosurgery.

I have had the opportunity use whānau support or a friend to help me ask questions and understand the study.

I understand that taking part in this study is voluntary (my choice) and that I may withdraw from the study at any time, and this will in no way affect my future health care and academic progress.

I understand that my participation in this study is confidential and that no material that could identify me will be used in any reports on this study.

I have had time to consider whether to take part in the study

I know whom to contact if I have side-effects from the study

I know whom to contact if I have any questions about the study in general

I consent to my interview being audiotaped

Yes

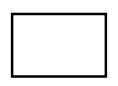

No

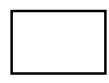

I wish to receive a copy of the results

Yes

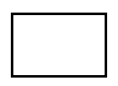

No

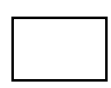


Date:

Signature:

Full names of researchers:

Contact phone number for researchers:

Project explained by:

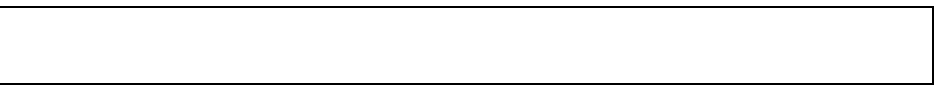

Project role:

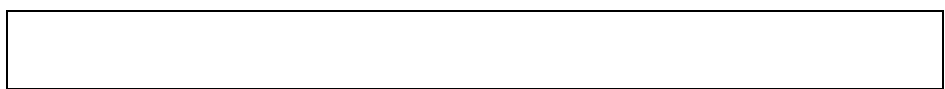

Signature:

Date: 
Appendix E: List of BLAST Stimuli

Table D.1

Frequency and Length of Items in Version 1 of the picture naming task in order of appearance

\begin{tabular}{|c|c|c|c|}
\hline Item & $\begin{array}{l}\text { CELEX Lemma } \\
\text { Frequency }\end{array}$ & $\begin{array}{c}\text { Frequency } \\
\text { Category }\end{array}$ & Syllable Length \\
\hline watch & 710 & hi & mono \\
\hline camel & 449 & hi & mono \\
\hline hamburger & 86 & med & poly \\
\hline dinosaur & 93 & med & poly \\
\hline apple & 546 & hi & mono \\
\hline strawberry & 115 & med & poly \\
\hline Kilt & 34 & lo & mono \\
\hline Saw & 62 & lo & mono \\
\hline monkey & 324 & hi & bi \\
\hline carrot & 144 & med & bi \\
\hline balloon & 112 & med & bi \\
\hline parachute & 7 & lo & poly \\
\hline ladder & 287 & hi & bi \\
\hline hospital & 2300 & hi & poly \\
\hline clown & 65 & lo & mono \\
\hline Nest & 304 & hi & mono \\
\hline cucumber & 85 & med & poly \\
\hline butter & 490 & hi & $\mathrm{bi}$ \\
\hline wheelbarrow & 22 & lo & poly \\
\hline pyramid & 123 & med & poly \\
\hline binoculars & 93 & med & poly \\
\hline hoof & 137 & med & mono \\
\hline guitar & 119 & med & bi \\
\hline shark & 357 & hi & mono \\
\hline scarecrow & 18 & lo & bi \\
\hline cigarette & 1274 & hi & poly \\
\hline astronaut & 50 & lo & poly \\
\hline crutch & 73 & lo & mono \\
\hline vegetables & 1050 & hi & poly \\
\hline tongs & 29 & lo & mono \\
\hline Crab & 170 & med & mono \\
\hline lipstick & 129 & med & bi \\
\hline chicken & 734 & hi & bi \\
\hline apron & 164 & med & bi \\
\hline caterpillar & 58 & lo & poly \\
\hline Owl & 128 & med & mono \\
\hline coconut & 51 & lo & poly \\
\hline finger & 2212 & hi & $\mathrm{bi}$ \\
\hline genie & 16 & lo & bi \\
\hline telescope & 142 & med & poly \\
\hline
\end{tabular}




\begin{tabular}{cccc}
\hline Item & $\begin{array}{c}\text { CELEX Lemma } \\
\text { Frequency }\end{array}$ & $\begin{array}{c}\text { Frequency } \\
\text { Category }\end{array}$ & Syllable Length \\
\hline whale & 199 & med & mono \\
tomatoes & 255 & hi & poly \\
cake & 610 & hi & mono \\
drill & 141 & med & mono \\
sandwich & 247 & hi & bi \\
reins & 13 & lo & mono \\
hippopotamus & 24 & lo & poly \\
envelope & 439 & hi & poly \\
Raft & 69 & lo & mono \\
banjo & 8 & lo & bi \\
well & 165 & med & mono \\
submarine & 311 & hi & poly \\
lighthouse & 50 & lo & bi \\
necklace & 71 & lo & bi \\
chair & 2441 & hi & mono \\
rhinoceros & 30 & lo & poly \\
cannon & 109 & med & bi \\
skirt & 522 & hi & mono \\
goat & 506 & hi & mono \\
igloo & 14 & lo & bi \\
\hline
\end{tabular}

Table D.2

Frequency and length of items in version 2 of the picture-naming task in order of appearance

\begin{tabular}{|c|c|c|c|}
\hline Item & $\begin{array}{l}\text { CELEX Lemma } \\
\text { Frequency }\end{array}$ & $\begin{array}{l}\text { Frequency } \\
\text { Category }\end{array}$ & Syllable Length \\
\hline flower & 1674 & hi & bi \\
\hline pocket & 1343 & hi & bi \\
\hline pillow & 344 & hi & bi \\
\hline stilts & 18 & lo & mono \\
\hline potato & 639 & hi & poly \\
\hline Waterfall* & 137 & med & poly \\
\hline refrigerator & 187 & med & poly \\
\hline typewritter & 2300 & hi & poly \\
\hline elephant & 429 & hi & poly \\
\hline pear & 112 & med & mono \\
\hline penguin & 90 & med & bi \\
\hline ambulance & 162 & med & poly \\
\hline library & 1113 & hi & poly \\
\hline pipe & 558 & hi & mono \\
\hline hammer & 197 & med & bi \\
\hline scissors & 79 & med & bi \\
\hline pencil & 332 & hi & bi \\
\hline volcano & 102 & med & poly \\
\hline wreath & 63 & lo & mono \\
\hline bottle & 2079 & hi & bi \\
\hline
\end{tabular}




\begin{tabular}{|c|c|c|c|}
\hline Item & $\begin{array}{l}\text { CELEX Lemma } \\
\text { Frequency }\end{array}$ & $\begin{array}{l}\text { Frequency } \\
\text { Category }\end{array}$ & Syllable Length \\
\hline cauliflower & 43 & lo & poly \\
\hline pumpkin & 38 & lo & $\mathrm{bi}$ \\
\hline jockey & 95 & med & bi \\
\hline veil & 166 & med & mono \\
\hline comb & 159 & med & mono \\
\hline stethoscope & 16 & lo & poly \\
\hline hammock & 19 & lo & bi \\
\hline swan & 134 & med & mono \\
\hline magnet & 52 & lo & bi \\
\hline sink & 892 & hi & mono \\
\hline funnel & 40 & lo & bi \\
\hline accordion & 18 & lo & poly \\
\hline sling & 63 & lo & mono \\
\hline microscope & 135 & med & poly \\
\hline ball & 1996 & hi & mono \\
\hline button & 468 & hi & bi \\
\hline earring & 59 & lo & bi \\
\hline desk & 1633 & hi & mono \\
\hline nun & 187 & med & mono \\
\hline banana & 151 & med & poly \\
\hline handkerchief & 351 & hi & poly \\
\hline dolphin & 54 & lo & $\mathrm{bi}$ \\
\hline flag & 461 & hi & mono \\
\hline calendar & 151 & med & poly \\
\hline helicopter & 281 & hi & poly \\
\hline snake & 412 & hi & mono \\
\hline whistle & 165 & med & bi \\
\hline buoy & 12 & hi & mono \\
\hline zip & 32 & lo & mono \\
\hline spider & 126 & med & bi \\
\hline asparagus & 38 & lo & poly \\
\hline octopus & 27 & lo & poly \\
\hline pendulum & 71 & lo & poly \\
\hline mop & 49 & lo & mono \\
\hline tusk & 33 & lo & mono \\
\hline handcuffs & 34 & lo & bi \\
\hline peg & 71 & med & mono \\
\hline triangle & 131 & med & poly \\
\hline mushroom & 227 & hi & $\mathrm{bi}$ \\
\hline vase & 127 & med & mono \\
\hline
\end{tabular}


Table D. 3

Frequency and length of items in version 3 of the picture-naming task in order of appearance

\begin{tabular}{|c|c|c|c|}
\hline Item & $\begin{array}{c}\text { CELEX Lemma } \\
\text { Frequency }\end{array}$ & $\begin{array}{l}\text { Frequency } \\
\text { Category }\end{array}$ & Syllable Length \\
\hline castle & 485 & hi & bi \\
\hline butcher & 112 & med & bi \\
\hline tent & 785 & hi & mono \\
\hline thermometer & 116 & med & poly \\
\hline net & 290 & hi & mono \\
\hline barrel & 379 & hi & bi \\
\hline giraffe & 28 & lo & bi \\
\hline cork & 98 & med & mono \\
\hline furniture & 696 & hi & poly \\
\hline butterfly & 183 & med & poly \\
\hline scarf & 219 & hi & mono \\
\hline pyjamas & 146 & med & poly \\
\hline buckle & 34 & lo & $\mathrm{bi}$ \\
\hline stool & 222 & hi & mono \\
\hline glasses & 571 & hi & bi \\
\hline trumpet & 140 & med & bi \\
\hline microphone & 152 & med & poly \\
\hline anchor & 102 & med & bi \\
\hline tail & 640 & hi & mono \\
\hline peacock & 69 & lo & bi \\
\hline tambourine & 13 & lo & poly \\
\hline spaghetti & 82 & med & poly \\
\hline mirror & 880 & hi & $\mathrm{bi}$ \\
\hline harp & 50 & lo & mono \\
\hline escalator & 30 & lo & poly \\
\hline zebra & 34 & lo & $\mathrm{bi}$ \\
\hline corn & 434 & hi & mono \\
\hline platypus & 22 & lo & poly \\
\hline reflection & 450 & hi & poly \\
\hline frog & 168 & med & mono \\
\hline calculator & 89 & med & poly \\
\hline dice & 38 & lo & mono \\
\hline umbrella & 245 & hi & poly \\
\hline star & 1804 & hi & mono \\
\hline feather & 379 & hi & bi \\
\hline skeleton & 210 & hi & poly \\
\hline turtle & 67 & lo & bi \\
\hline hose & 72 & lo & mono \\
\hline computer & 1683 & hi & poly \\
\hline newspaper & 2176 & hi & poly \\
\hline canoe & 101 & med & $\mathrm{bi}$ \\
\hline tripod & 25 & lo & bi \\
\hline shadow & 929 & hi & bi \\
\hline windmill & 159 & med & bi \\
\hline
\end{tabular}




\begin{tabular}{cccc}
\hline Item & $\begin{array}{c}\text { CELEX Lemma } \\
\text { Frequency }\end{array}$ & $\begin{array}{c}\text { Frequency } \\
\text { Category }\end{array}$ & Syllable Length \\
rake & 33 & lo & mono \\
cherry & 132 & med & bi \\
Eskimo & 31 & lo & poly \\
saddle & 177 & med & bi \\
dart & 57 & lo & mono \\
pineapple & 53 & lo & poly \\
ostrich & 48 & lo & bi \\
snail & 80 & med & mono \\
television & 2043 & hi & poly \\
skunk & 4 & lo & mono \\
gorilla & 54 & lo & poly \\
plug & 170 & med & mono \\
kangaroo & 48 & lo & poly \\
safe & 127 & med & mono \\
kite & 83 & med & mono \\
hood & 106 & med & mono \\
\hline
\end{tabular}

Table D.4

Selection strength ratios and frequencies for items in version 1 of the verb generation task in order of appearance

\begin{tabular}{ccccc}
\hline Item & $\begin{array}{c}\text { Selection } \\
\text { Strength Ratio }\end{array}$ & $\begin{array}{c}\text { Selection } \\
\text { Strength } \\
\text { Category }\end{array}$ & $\begin{array}{c}\text { LEMMA } \\
\text { Frequency } \\
\text { Value }\end{array}$ & $\begin{array}{c}\text { Frequency } \\
\text { Category }\end{array}$ \\
\hline barbeque & 1.38 & weak & 39 & lo \\
van & 10 & strong & 1034 & hi \\
crane & 10 & strong & 71 & lo \\
tail & 2.17 & weak & 640 & hi \\
razor & 1.8 & weak & 156 & lo \\
stethoscope & 6.4 & strong & 16 & lo \\
penny & 2 & weak & 476 & hi \\
curtains & 2 & weak & 784 & hi \\
baby & 1.6 & weak & 4620 & hi \\
heart & 2.43 & weak & 2937 & hi \\
ice & 1.21 & weak & 944 & hi \\
nun & 41 & strong & 187 & hi \\
bed & 10.33 & strong & 4831 & lo \\
shark & 1.21 & weak & 357 & lo \\
axe & 1.05 & weak & 153 & lo \\
yacht & 18.5 & strong & 108 & lo \\
mosquito & 7.75 & strong & 96 & lo \\
wool & 11 & strong & 384 & hi \\
piano & 40 & strong & 488 & lo \\
ladder & 40 & strong & 287 & hi \\
stomach & 5.4 & strong & 769 &
\end{tabular}




\begin{tabular}{|c|c|c|c|c|}
\hline Item & $\begin{array}{c}\text { Selection } \\
\text { Strength Ratio }\end{array}$ & $\begin{array}{l}\text { Selection } \\
\text { Strength } \\
\text { Category }\end{array}$ & $\begin{array}{c}\text { LEMMA } \\
\text { Frequency } \\
\text { Value }\end{array}$ & $\begin{array}{l}\text { Frequency } \\
\text { Category }\end{array}$ \\
\hline duck & 2.17 & weak & 248 & 10 \\
\hline hinge & 2.67 & weak & 64 & lo \\
\hline ear & 2.07 & weak & 1570 & hi \\
\hline worm & 2.8 & weak & 302 & lo \\
\hline lion & 39 & strong & 454 & hi \\
\hline boat & 1.38 & weak & 1386 & hi \\
\hline fire & 8 & strong & 2905 & hi \\
\hline ball & 4.6 & strong & 1996 & hi \\
\hline pool & 13.5 & strong & 733 & hi \\
\hline towel & 40 & strong & 392 & lo \\
\hline trapeze & 5 & strong & 9 & lo \\
\hline tongue & 1.44 & weak & 715 & hi \\
\hline airplane & 12.67 & strong & 102 & lo \\
\hline bell & 11.67 & strong & 745 & hi \\
\hline fence & 1.2 & weak & 537 & hi \\
\hline sparrow & 1.5 & weak & 79 & lo \\
\hline kettle & 19.5 & strong & 216 & lo \\
\hline sugar & 6.75 & strong & 1015 & hi \\
\hline crab & 1.11 & weak & 170 & lo \\
\hline chair & 11.33 & strong & 2441 & hi \\
\hline picture & 5.2 & strong & 3113 & hi \\
\hline feet & 1.73 & weak & 5857 & hi \\
\hline caravan & 1.8 & weak & 179 & lo \\
\hline leg & 1.36 & weak & 3140 & hi \\
\hline radio & 1.64 & weak & 1582 & hi \\
\hline
\end{tabular}

Table D.5

Selection strength ratios and frequencies for items in version 2 of the verb generation task in order of appearance

\begin{tabular}{ccccc}
\hline Item & $\begin{array}{c}\text { Selection } \\
\text { Strength Ratio }\end{array}$ & $\begin{array}{c}\text { Selection } \\
\text { Strength } \\
\text { Category }\end{array}$ & $\begin{array}{c}\text { LEMMA } \\
\text { Frequency } \\
\text { Value }\end{array}$ & $\begin{array}{c}\text { Frequency } \\
\text { Category }\end{array}$ \\
\hline elbow & 17.5 & strong & 466 & $\mathrm{hi}$ \\
church & 10 & strong & 3287 & $\mathrm{hi}$ \\
arrow & 12 & strong & 264 & lo \\
telephone & 18 & strong & 1876 & $\mathrm{hi}$ \\
scissors & $100 \%$ response & strong & 79 & lo \\
pills & agreement & weak & 507 & $\mathrm{hi}$ \\
stove & 1.21 & strong & 364 & lo \\
road & 19.5 & weak & 4458 & $\mathrm{hi}$ \\
daisy & 1.14 & weak & 568 & $\mathrm{hi}$ \\
watch & 1.23 & weak & 710 & $\mathrm{hi}$
\end{tabular}




\begin{tabular}{|c|c|c|c|c|}
\hline Item & $\begin{array}{c}\text { Selection } \\
\text { Strength Ratio }\end{array}$ & $\begin{array}{l}\text { Selection } \\
\text { Strength } \\
\text { Category }\end{array}$ & $\begin{array}{c}\text { LEMMA } \\
\text { Frequency } \\
\text { Value }\end{array}$ & $\begin{array}{c}\text { Frequency } \\
\text { Category }\end{array}$ \\
\hline basket & 13 & strong & 428 & 10 \\
\hline key & 1.5 & weak & 1544 & hi \\
\hline sun & 9.67 & strong & 2728 & hi \\
\hline rope & 5.2 & strong & 745 & hi \\
\hline pipe & 20 & strong & 558 & hi \\
\hline alligator & 2.78 & weak & 28 & lo \\
\hline shovel & $\begin{array}{c}100 \% \text { response } \\
\text { agreement }\end{array}$ & strong & 76 & lo \\
\hline dice & 6.8 & strong & 16 & lo \\
\hline binoculars & 2.1 & weak & 9 & 10 \\
\hline can & 11 & strong & 166 & lo \\
\hline teeth & 18 & strong & 56 & lo \\
\hline pan & 2.78 & strong & 489 & hi \\
\hline hawk & 5.25 & strong & 109 & lo \\
\hline scales & 6.4 & strong & 1479 & hi \\
\hline frog & 1 & weak & 8 & 10 \\
\hline cigarette & 2.89 & weak & 1274 & hi \\
\hline package & 1.14 & weak & 357 & lo \\
\hline needle & 1.83 & weak & 294 & lo \\
\hline tiger & 2.5 & weak & 214 & lo \\
\hline horse & 7.33 & strong & 2372 & hi \\
\hline straw & 1.8 & weak & 461 & hi \\
\hline envelope & 1.1 & weak & 83 & lo \\
\hline ghost & 5.75 & strong & 554 & hi \\
\hline broom & 13 & strong & 140 & lo \\
\hline seesaw & 1.8 & weak & 12 & lo \\
\hline soldier & 1.15 & weak & 1488 & hi \\
\hline candle & 1.38 & weak & 294 & lo \\
\hline lips & 1.78 & weak & 1401 & hi \\
\hline towel & 40 & strong & 392 & lo \\
\hline moon & 2.57 & weak & 1058 & hi \\
\hline priest & 17.5 & strong & 873 & hi \\
\hline carnation & 2.13 & weak & 28 & lo \\
\hline snow & 6.5 & strong & 1102 & hi \\
\hline basin & 9 & strong & 341 & lo \\
\hline suitcase & 1.5 & weak & 334 & lo \\
\hline
\end{tabular}


Table D.6

Frequencies and syllable length for version 1 of the picture-word verification task in order of appearance

\begin{tabular}{|c|c|c|c|c|}
\hline Target & Distractor & $\begin{array}{c}\text { Distractor } \\
\text { Type }\end{array}$ & $\begin{array}{c}\text { Frequency } \\
\text { (Log CELEX } \\
\text { lemma } \\
\text { frequencies) }\end{array}$ & Syllable Length \\
\hline scissors & curler & UR & 0.78 & 2 \\
\hline cannon & cattle & Phon & 2.83 & 2 \\
\hline bread & toast & Sem & 3.23 & 1 \\
\hline scissors & scissors & Rel & 2.53 & 2 \\
\hline cat & can & Phon & 5.43 & 1 \\
\hline spider & spiral & Phon & 1.96 & 2 \\
\hline cannon & cannon & Rel & 2.65 & 2 \\
\hline spoon & file & UR & 3.35 & 1 \\
\hline rabbit & cradle & UR & 2.16 & 2 \\
\hline salad & satin & Phon & 2.13 & 2 \\
\hline pencil & pencil & Rel & 2.70 & 2 \\
\hline salad & vessel & UR & 2.68 & 2 \\
\hline candle & whisker & UR & 1.15 & 2 \\
\hline spoon & spear & Phon & 2.37 & 1 \\
\hline drum & drip & Phon & 2.42 & 1 \\
\hline pencil & chalk & Sem & 2.26 & 1 \\
\hline cannon & pistol & Sem & 2.71 & 2 \\
\hline drum & corn & UR & 2.86 & 1 \\
\hline bread & bread & Rel & 3.16 & 1 \\
\hline candle & torch & Sem & 4.98 & 1 \\
\hline rabbit & rabbit & Rel & 3.01 & 2 \\
\hline spider & gherkin & UR & 0.70 & 2 \\
\hline scissors & dagger & Sem & 4.92 & 2 \\
\hline pencil & pendant & Phon & 1.67 & 2 \\
\hline pizza & peeler & Phon & 1.20 & 2 \\
\hline spoon & mug & Sem & 6.54 & 1 \\
\hline drum & bass & Sem & 2.59 & 1 \\
\hline bread & form & UR & 3.34 & 1 \\
\hline cannon & chin & UR & 2.81 & 1 \\
\hline cat & cat & Rel & 3.53 & 1 \\
\hline spider & spider & Rel & 2.71 & 2 \\
\hline candle & canvas & Phon & 2.34 & 2 \\
\hline rabbit & beaver & Sem & 2.39 & 2 \\
\hline salad & pasta & Sem & 2.34 & 2 \\
\hline pizza & burger & Sem & 2.72 & 2 \\
\hline pencil & drama & UR & 3.01 & 2 \\
\hline cat & frost & UR & 2.39 & 1 \\
\hline bread & brain & Phon & 3.59 & 1 \\
\hline pizza & gecko & UR & 1.3 & 2 \\
\hline rabbit & rabbi & Phon & 2.54 & 1 \\
\hline
\end{tabular}




\begin{tabular}{ccccc}
\hline Target & Distractor & $\begin{array}{c}\text { Distractor } \\
\text { Type }\end{array}$ & $\begin{array}{c}\text { Frequency } \\
\text { (Log CELEX } \\
\text { lemma } \\
\text { frequencies) }\end{array}$ & Syllable Length \\
\hline salad & salad & Rel & 2.94 & 2 \\
spoon & spoon & Rel & 2.59 & 1 \\
drum & drum & Rel & 2.64 & 1 \\
scissors & syrup & Phon & 5.1 & 2 \\
spider & cockroach & Sem & 2.24 & 2 \\
pizza & pizza & Rel & 3.23 & 1 \\
candle & candle & Rel & 2.61 & 2 \\
cat & lamb & Sem & 2.73 & 1 \\
\hline
\end{tabular}

Table D.7

Frequencies and syllable length for version 2 of the picture-word verification task in order of appearance

\begin{tabular}{|c|c|c|c|c|}
\hline Target & Distractor & $\begin{array}{c}\text { Distractor } \\
\text { Type }\end{array}$ & $\begin{array}{c}\text { Frequency } \\
\text { (Log CELEX } \\
\text { lemma } \\
\text { frequencies }\end{array}$ & Syllable Length \\
\hline trumpet & violin & Sem & 2.39 & 2 \\
\hline grapes & grease & Phon & 2.55 & 1 \\
\hline hammer & pearl & UR & 2.90 & 1 \\
\hline knife & $\operatorname{limb}$ & UR & 2.38 & 1 \\
\hline trumpet & truffle & Phon & 1.38 & 2 \\
\hline horse & deer & Sem & 2.65 & 1 \\
\hline chair & stool & Sem & 2.26 & 1 \\
\hline lemon & leather & Phon & 2.84 & 2 \\
\hline grapes & puzzle & UR & 2.57 & 2 \\
\hline razor & perfume & UR & 2.77 & 2 \\
\hline turkey & turkey & Rel & 3.06 & 2 \\
\hline knife & fork & Sem & 2.65 & 1 \\
\hline carrot & spinach & Sem & 2.12 & 2 \\
\hline monkey & monkey & Rel & 3.23 & 2 \\
\hline lemon & lemon & Rel & 2.79 & 2 \\
\hline trumpet & denim & UR & 1.53 & 2 \\
\hline carrot & carrot & Rel & 2.29 & 2 \\
\hline trumpet & trumpet & Rel & 2.32 & 2 \\
\hline horse & linen & UR & 2.18 & 2 \\
\hline arrow & torch & UR & 2.41 & 1 \\
\hline chair & chair & Rel & 3.40 & 1 \\
\hline razor & radar & Phon & 3.26 & 2 \\
\hline monkey & emerald & UR & 2.12 & 3 \\
\hline arrow & Arab & Phon & 2.24 & 1 \\
\hline chair & nickel & UR & 2.64 & 2 \\
\hline turkey & star & UR & 3.62 & 1 \\
\hline
\end{tabular}




\begin{tabular}{ccccc}
\hline Target & Distractor & $\begin{array}{c}\text { Distractor } \\
\text { Type }\end{array}$ & $\begin{array}{c}\text { Frequency } \\
\text { (Log CELEX } \\
\text { lemma }\end{array}$ & Syllable Length \\
frequencies & \\
\hline grapes & peach & Sem & 2.51 & 1 \\
horse & horse & Rel & 3.68 & 1 \\
lemon & scarf & UR & 2.38 & 1 \\
monkey & panda & Sem & 2.04 & 2 \\
razor & razor & Rel & 2.54 & 2 \\
arrow & arrow & Rel & 2.60 & 2 \\
hammer & axe & Sem & 2.4 & 1 \\
chair & check & Phon & 4.15 & 1 \\
monkey & mustard & Phon & 2.52 & 2 \\
turkey & duck & Sem & 3.1 & 1 \\
knife & knife & Rel & 3.38 & 2 \\
turkey & turban & Phon & 1.83 & 2 \\
lemon & orange & Sem & 3.06 & 1 \\
knife & nine & Phon & 3.54 & 1 \\
grapes & grapes & Rel & 2.31 & 2 \\
hammer & hamlet & Phon & 2.37 & 1 \\
arrow & pipe & UR & 3.00 & 1 \\
carrot & chasm & Phon & 1.28 & 1 \\
horse & haunt & Phon & 2.26 & 2 \\
hammer & hammer & Rel & 2.80 & \\
\hline
\end{tabular}

Table D.8

Frequencies and imageability of the single word repetition task in order of appearance

\begin{tabular}{cccccc}
\hline Item & Freq_KF & LogFreq_KF & $\begin{array}{c}\text { Freq } \\
\text { Category }\end{array}$ & Imageability & $\begin{array}{c}\text { Imageability } \\
\text { Category }\end{array}$ \\
\hline episode & 12 & 1.08 & Lo & 370 & Lo \\
theory & 129 & 2.11 & Hi & 317 & Lo \\
potato & 15 & 1.18 & Lo & 617 & Hi \\
church & 348 & 2.54 & Hi & 616 & Hi \\
folly & 10 & 1.00 & Lo & 326 & Lo \\
irony & 12 & 1.08 & Lo & 293 & Lo \\
battle & 87 & 1.94 & Hi & 597 & Hi \\
concept & 85 & 1.93 & Hi & 258 & Lo \\
spider & 2 & 0.30 & Lo & 597 & Hi \\
village & 72 & 1.86 & Hi & 578 & Hi \\
deed & 8 & 0.90 & Lo & 390 & Lo \\
gravy & 4 & 0.60 & Lo & 594 & Hi \\
dogma & 4 & 0.60 & Lo & 327 & Lo \\
alcohol & 13 & 1.11 & Lo & 598 & Hi \\
picture & 162 & 2.21 & Hi & 581 & Hi \\
radio & 120 & 2.08 & Hi & 613 & Hi
\end{tabular}




\begin{tabular}{|c|c|c|c|c|c|}
\hline Item & Freq_KF & LogFreq_KF & $\begin{array}{c}\text { Freq } \\
\text { Category }\end{array}$ & Imageability & $\begin{array}{c}\text { Imageability } \\
\text { Category }\end{array}$ \\
\hline onion & 15 & 1.18 & Lo & 617 & $\mathrm{Hi}$ \\
\hline purpose & 149 & 2.17 & Hi & 280 & Lo \\
\hline quality & 114 & 2.06 & $\mathrm{Hi}$ & 349 & Lo \\
\hline school & 492 & 2.69 & $\mathrm{Hi}$ & 599 & $\mathrm{Hi}$ \\
\hline system & 416 & 2.62 & $\mathrm{Hi}$ & 340 & Lo \\
\hline elephant & 7 & 0.85 & Lo & 616 & $\mathrm{Hi}$ \\
\hline pig & 8 & 0.90 & Lo & 635 & $\mathrm{Hi}$ \\
\hline night & 411 & 2.61 & $\mathrm{Hi}$ & 607 & Hi \\
\hline marriage & 95 & 1.98 & $\mathrm{Hi}$ & 556 & $\mathrm{Hi}$ \\
\hline thing & 333 & 2.52 & $\mathrm{Hi}$ & 358 & Lo \\
\hline bonus & 2 & 0.30 & Lo & 397 & Lo \\
\hline opinion & 96 & 1.98 & $\mathrm{Hi}$ & 359 & Lo \\
\hline analogy & 13 & 1.11 & Lo & 267 & Lo \\
\hline hand & 431 & 2.63 & $\mathrm{Hi}$ & 598 & $\mathrm{Hi}$ \\
\hline woe & 5 & 0.70 & Lo & 348 & Lo \\
\hline character & 118 & 2.07 & $\mathrm{Hi}$ & 372 & Lo \\
\hline wheat & 9 & 0.95 & Lo & 577 & $\mathrm{Hi}$ \\
\hline effort & 145 & 2.16 & $\mathrm{Hi}$ & 367 & Lo \\
\hline tribute & 24 & 1.38 & Lo & 386 & Lo \\
\hline fact & 447 & 2.65 & $\mathrm{Hi}$ & 302 & Lo \\
\hline valour & N/A & - & Lo & - & Lo \\
\hline idea & 195 & 2.29 & Hi & 319 & Lo \\
\hline axe & 6 & 0.78 & $\mathrm{Hi}$ & 597 & $\mathrm{Hi}$ \\
\hline funnel & 1 & 0.00 & Lo & - & $\mathrm{Hi}$ \\
\hline tractor & 24 & 1.38 & Lo & 585 & $\mathrm{Hi}$ \\
\hline length & 116 & 2.06 & $\mathrm{Hi}$ & 395 & Lo \\
\hline plea & 11 & 1.04 & Lo & 347 & Lo \\
\hline monkey & 9 & 0.95 & Lo & 588 & $\mathrm{Hi}$ \\
\hline manner & 124 & 2.09 & $\mathrm{Hi}$ & 342 & Lo \\
\hline satire & 9 & 0.95 & Lo & 370 & Lo \\
\hline drum & 11 & 1.04 & Lo & 599 & $\mathrm{Hi}$ \\
\hline cart & 5 & 0.70 & Lo & 597 & $\mathrm{Hi}$ \\
\hline miracle & 16 & 1.20 & Lo & 367 & Lo \\
\hline hospital & 110 & 2.04 & $\mathrm{Hi}$ & 60 & $\mathrm{Hi}$ \\
\hline audience & 115 & 2.06 & $\mathrm{Hi}$ & 555 & $\mathrm{Hi}$ \\
\hline attitude & 8 & 0.90 & Lo & 321 & Lo \\
\hline letter & 145 & 2.16 & Hi & 595 & Hi \\
\hline tobacco & 19 & 1.28 & Lo & 601 & $\mathrm{Hi}$ \\
\hline principle & 109 & 2.04 & $\mathrm{Hi}$ & 305 & Lo \\
\hline plane & 114 & 2.06 & $\mathrm{Hi}$ & 556 & $\mathrm{Hi}$ \\
\hline moment & 246 & 2.39 & Hi & 334 & Lo \\
\hline
\end{tabular}




\begin{tabular}{cccccc}
\hline Item & Freq_KF & LogFreq_KF & $\begin{array}{c}\text { Freq } \\
\text { Category }\end{array}$ & Imageability & $\begin{array}{c}\text { Imageability } \\
\text { Category }\end{array}$ \\
\hline summer & 134 & 2.13 & Hi & 618 & Hi \\
feather & 6 & 0.78 & Lo & - & Hi \\
pact & 5 & 0.70 & Lo & 364 & Lo \\
\hline
\end{tabular}

Table D.9

List of items in the nonword repetition task and their associated actual word, in order of appearance.

\begin{tabular}{cc}
\hline Nonword & Associated Word \\
\hline biffle & battle \\
ragio & radio \\
clee & deed \\
otion & onion \\
drim & drum \\
sping & thing \\
slurch & student \\
plen & plea \\
atalogy & analogy \\
parpise & purpose \\
loment & moment \\
lutter & letter \\
hend & hand \\
trantor & tractor \\
voe & woe \\
merly & mercy \\
baranter & character \\
affort & effort \\
gramy & gravy \\
spunder & spider \\
\hline
\end{tabular}

Table D.10

Items, their associated colours and condition in the Stroop task in order of appearance

\begin{tabular}{ccc}
\hline Item & Colour & Condition \\
\hline pink & pink & Congruent \\
brown & grey & Incongruent \\
red & red & Congruent \\
purple & yellow & Incongruent \\
green & orange & Incongruent \\
blue & purple & Incongruent
\end{tabular}




\begin{tabular}{ccc}
\hline Item & Colour & Condition \\
\hline orange & orange & Congruent \\
yellow & blue & Incongruent \\
purple & red & Incongruent \\
blue & blue & Congruent \\
red & green & Incongruent \\
green & yellow & Incongruent \\
blue & green & Incongruent \\
orange & purple & Incongruent \\
yellow & yellow & Congruent \\
red & blue & Incongruent \\
purple & purple & Congruent \\
orange & red & Incongruent \\
green & green & Congruent \\
yellow & orange & Incongruent \\
\hline
\end{tabular}

Table D.11

Items presented in order of appearance in the Articulatory Agility test

\begin{tabular}{cc}
\hline Item \\
\hline mamma \\
tip-top \\
fifty-fifty \\
thanks \\
huckleberry \\
baseball player \\
caterpillar \\
\hline
\end{tabular}

Table D.12

Frequency and imageability for section 1 of the reading test in order of appearance

\begin{tabular}{cccc}
\hline Item & Block & LogFreq_KF & Imageability \\
\hline key & 1 & 2.44 & 6.39 \\
fox & 1 & 1.11 & 6.27 \\
car & 1 & 1.94 & 6.49 \\
ship & 2 & 1.91 & 6.35 \\
book & 2 & 1.91 & 6.35 \\
bird & 2 & 2.14 & 6.00 \\
dress & 3 & 1.83 & 6.08
\end{tabular}




\begin{tabular}{cccc}
\hline Item & Block & LogFreq_KF & Imageability \\
\hline smoke & 3 & 1.53 & 6.41 \\
heart & 3 & 2.24 & 6.24 \\
bridge & 4 & 1.99 & 6.14 \\
letter & 4 & 2.16 & 6.35 \\
square & 4 & 1.52 & 6.16 \\
\hline
\end{tabular}

Table D.13

Frequency, imageability and regularity of section 2 of the reading task in order of appearance

\begin{tabular}{cccccc}
\hline Item & Frequency & Freq_KF & Imageability & $\begin{array}{c}\text { Imageability } \\
\text { Rating }\end{array}$ & Regularity \\
\hline worm & lo & 4 & hi & 578 & Irregular \\
fraud & lo & 5 & lo & 381 & Regular \\
yacht & lo & 4 & hi & 624 & Irregular \\
hand & hi & 431 & hi & 598 & Regular \\
horse & hi & 117 & hi & 624 & Regular \\
trout & lo & 4 & hi & 617 & Regular \\
break & hi & 88 & lo & 398 & Irregular \\
vest & lo & 4 & hi & 581 & Regular \\
cause & hi & 130 & lo & 282 & Regular \\
side & hi & 380 & lo & 386 & Regular \\
give & hi & 391 & lo & 383 & Irregular \\
plead & lo & 5 & lo & 393 & Regular \\
head & hi & 424 & hi & 593 & Irregular \\
shone & lo & 5 & lo & 384 & Irregular \\
dread & lo & 9 & lo & 378 & Irregular \\
blood & hi & 121 & hi & 620 & Irregular \\
\hline
\end{tabular}


Table D.14

Items Presented in Order of Appearance for the Nonword Reading Task

\section{Item}

ked

nar

fon

shid

doop

dusp

snite

hoach

glope

dringe

churse

shoave 


\section{References}

“Brain Cancer: Gliobastoma multiforme (GBM)." (2013) Retrieved April 18, 2014, from http://www.cancernz.co.nz/brain-cancer/

Adlam, A., L. R., Patterson, K., Rogers, T. T., Nestor, P. J., Salmond, C. H., AcostaCabronero, J., \& Hodges, J. R. (2006). Semantic dementia and fluent primary progressive aphasia: two sides of the same coin? Brain : A Journal of Neurology, 129(11), 3066-3080. doi:10.1016/s0513-5117(08)79049-5

Adlam, A.L. R., Patterson, K., Rogers, T. T., Nestor, P. J., Salmond, C. H., AcostaCabronero, J., \& Hodges, J. R. (2006). Semantic dementia and fluent primary progressive aphasia: two sides of the same coin? Brain : A Journal of Neurology, 129(11), 3066-3080. doi:10.1016/s0513-5117(08)79049-5

Ahrens, K. (2003). Verbal integration: The interaction of participant roles and sentential arguments. Journal of Psycholinguistic Research, 32(5), 497-516. doi:10.1023/A:1025452814385

Ali, M. Z., Fadel, N. A., \& Abouldahab, H. A. (2009). Awake craniotomy versus general anaesthesia for managing eloquent cortex low-grade gliomas. Neurosciences (Riyadh, Saudi Arabia), 14(3), 263-272. Doi:10.1227/neu.0b013e31820c02a3

Anderson, S. W., Damasio, H., \& Tranel, D. (1990). Neuropsychological impairments associated with lesions caused by tumor or stroke. Archives of Neurology, 47(4), 397405. doi:10.1001/archneur.1990.00530040039017

Andreetta, S., Cantagallo, A., \& Marini, A. (2012). Narrative discourse in anomic aphasia. Neuropsychologia, 50(8), 1787-1793. doi:10.1016/j.neuropsychologia.2012.04.003

Arber, A., Faithfull, S., Plaskota, M., Lucas, C., \& de Vries, K. (2010). A study of patients with a primary malignant brain tumour and their carers: symptoms and access to 
services. International Journal of Palliative Nursing, 16(1), 24-30.

doi:10.12968/ijpn.2010.16.1.46180

Archibald, L. M. D., \& Gathercole, S. E. (2007). Nonword repetition and serial recall: Equivalent measures of verbal short-term memory? Applied PsychoLinguistics, 28(04), 587-606. doi:10.1017/s0142716407070324

Ash, S., Moore, P., Antani, S., McCawley, G., Work, M., \& Grossman, M. (2006). Trying to tell a tale: discourse impairments in progressive aphasia and frontotemporal dementia. Neurology, 66(9), 1405-1413. doi:10.1212/01.wnl.0000210435.72614.38

Ashburner, J., \& Friston, K. J. (2000). Voxel-based morphometry - the methods. Neuroimage, 11(6), 805-821. doi:10.1006/nimg.2000.0582

Avila, C., Lambon Ralph, M. A., Parcet, M. A., Geffner, D., \& Gonzalez-Darder, J. M. (2001). Implicit word cues facilitate impaired naming performance: Evidence from a case of anomia. Brain and Language, 79(2), 185-200. doi:10.1006/brln.2001.2472

Baayen, R. H., Piepenbrock, R., \& van H, R. (1993). The \{CELEX\} lexical database on $\{\mathrm{CD}-\mathrm{ROM}\}$.

Baddeley, A., Lewis, V., \& Vallar, G. (1984). Exploring the articulatory loop. The Quarterly journal of experimental psychology, 36(2), 233-252.

doi:10.1080/14640748408402157

Badre, D. (2008). Cognitive control, hierarchy, and the rostro-caudal organization of the frontal lobes. Trends in cognitive sciences, 12(5), 193-200. doi:

10.1016/j.tics.2008.02.004

Badre, D., \& D'Esposito, M. (2007). Functional magnetic resonance imaging evidence for a hierarchical organization of the prefrontal cortex. Cognitive Neuroscience, Journal of, 19(12), 2082-2099. doi: 10.1162/jocn.2007.91201 
Badre, D., \& Wagner, A. D. (2007). Left ventrolateral prefrontal cortex and the cognitive control of memory. Neuropsychologia, 45(13), 2883-2901. doi:

10.1016/j.neuropsychologia.2007.06.015

Badre, D., Poldrack, R. A., Paré-Blagoev, E. J., Insler, R. Z., \& Wagner, A. D. (2005). Dissociable controlled retrieval and generalized selection mechanisms in ventrolateral prefrontal cortex. Neuron, 47(6), 907-918. doi: 10.1016/j.neuron.2005.07.023

Baldo, J. V, \& Shimamura, A. P. (1998). Letter and category fluency in patients with frontal lobe lesions. Neuropsychology, 12(2), 259-267. doi:10.1037/0894-4105.12.2.259

Baldo, J. V, Katseff, S., \& Dronkers, N. F. (2012). Brain regions underlying repetition and auditory-verbal short-term memory deficits in aphasia: Evidence from voxel-based lesion symptom mapping. Aphasiology, 26(3-4), 338-354.

doi:10.1080/02687038.2011.602391

Baldo, J. V, Schwartz, S., Wilkins, D., \& Dronkers, N. F. (2006). Role of frontal versus temporal cortex in verbal fluency as revealed by voxel-based lesion symptom mapping. Journal of the International Neuropsychological Society JINS, 12(6), 896900. doi:10.1017/s1355617706061078

Baldo, J. V., Arévalo, A., Patterson, J. P., \& Dronkers, N. F. (2013). Grey and white matter correlates of picture naming: evidence from a voxel-based lesion analysis of the Boston Naming Test. Cortex, 49(3), 658-667. doi:10.1016/j.cortex.2012.03.001

Baldo, J. V., Wilson, S. M., \& Dronkers, N. F. (2012). Uncovering the neural substrates of language: A voxel-based lesion symptom mapping approach. Advances in the Neural Substrates of Language: Toward a Synthesis of Basic Science and Clinical Research. Oxford: Wiley-Blackwell. 
Balota, D. A., Yap, M. J., Hutchison, K. A., Cortese, M. J., Kessler, B., Loftis, B., ... \& Treiman, R. (2007). The English lexicon project. Behavior Research Methods, 39(3), 445-459. Retrieved from http://elexicon.wustl.edu/userguide.pdf

Barton J. J. S., Hanif H. M., Björnström L. E., \& Hills C. (2014). The word length effect in reading: A review. Cognitive Neuropsychology, 31, 378-412. doi: $10.1080 / 02643294.2014 .895314$

Basso, A., Casati, G., \& Vignolo, L. A. (1977). Phonemic identification defect in aphasia. Cortex; a Journal Devoted to the Study of the Nervous System and Behavior, 13(1), 85-95. doi:10.1016/S0010-9452(77)80057-9

Bates, E., Wilson, S. M., Saygin, A. P., Dick, F., Sereno, M. I., Knight, R. T., \& Dronkers, N. F. (2003). Voxel-based lesion-symptom mapping. Nature Neuroscience, 6(5), 448450. doi:10.1038/nn1050

Baum, S. R. (2002). Sensitivity to sub-syllabic constituents in brain-damaged patients: Evidence from word games. Brain and Language, 83(2), 237-248. doi:10.1016/s0093-934x(02)00034-2

Beauvois, M. F., \& Derouesne, J. (1979). Phonological alexia: three dissociations. Journal of Neurology, Neurosurgery \& Psychiatry, 42(12), 1115-1124. doi:10.1093/neucas/1.3.251

Behin, A., Hoang-Xuan, K., Carpentier, A., \& Delattre, J. (2003). Primary brain tumours in adults. Lancet, 361(9354), 323-331. Retrieved from http://www.ncbi.nlm.nih.gov/entrez/query.fcgi? cmd $=$ Retrieve $\& d b=$ PubMed\&dopt $=C$ itation\&list_uids $=12559880$

Behrmann, M., \& Bub, D. (1992). Surface dyslexia and dysgraphia: Dual routes, single lexicon. Cognitive Neuropsychology, 9(3), 209-251. doi:10.1080/02643299208252059 
Behrmann, M., Black, S. E., \& Bub, D. (1990). The evolution of pure alexia: a longitudinal study of recovery. Brain and language, 39(3), 405-427. doi:10.1016/0093934X(90)90148-A

Behrmann, M., Shomstein, S. S., Black, S. E., \& Barton, J. J. (2001). The eye movements of pure alexic patients during reading and nonreading tasks. Neuropsychologia, 39(9), 983-1002. doi:10.1016/s0028-3932(01)00021-5

Bello, L., Gallucci, M., Fava, M., Carrabba, G., Giussani, C., Acerbi, F., ... Gaini, S. M. (2007). Intraoperative subcortical language tract mapping guides surgical removal of gliomas involving speech areas. Neurosurgery, 60(1), 67-80. doi:10.1227/00006123200608000-00140

Benton, A. L. (1969). Development of a multilingual aphasia battery: Progress and problems. Journal of the neurological sciences, 9(1), 39-48. doi:10.1016/0022-510x(69)90057-4

Benton, A. L. (Ed.). (1994). Contributions to neuropsychological assessment: A clinical manual. Oxford University Press.

Berndt, R. S. (2000) Quantitative production analysis: A training manual for the analysis of aphasic sentence production. Psychology Press, 2000.

Berthier, M. L. (2005). Poststroke aphasia. Drugs \& aging, 22(2), 163-182. Retrieved from http://www.sciencemag.org/content/310/5755/1797.short

Biegler, K. A., Crowther, J. E., \& Martin, R. C. (2008). Consequences of an inhibition deficit for word production and comprehension: evidence from the semantic blocking paradigm. Cognitive Neuropsychology, 25(4), 493-527. doi:10.1080/02643290701862316

Bishop, D. V. M. (2003). The test for Reception for Grammar, version 2 (TROG-2). London: Psychological Corporation. 
Black, M., \& Chiat, S. (2003). Noun-verb dissociations: A multi-faceted phenomenon. Journal of Neurolinguistic, 16(2), 231-250. doi:10.1016/s0911-6044(02)00017-9

Blumstein, S. E., Baker, E., \& Goodglass, H. (1977). Phonological factors in auditory comprehension in aphasia. Neuropsychologia, 15(1), 19-30. doi:10.1016/00283932(77)90111-7

Borovsky, A., Saygin, A. P., Bates, E., \& Dronkers, N. (2007). Lesion correlates of conversational speech production deficits. Neuropsychologia, 45(11), 2525-2533. doi: 10.1016/j.neuropsychologia.2007.03.023

Bosman, F., Carneiro, F., Hruban, R., \& Theise, N. (2010). WHO Classification of Tumours of the Digestive System. (F. T. Bosman, F. Carneiro, R. H. Hruban, \& N. D. Theise, Eds.) World Health Organization classification of tumours (p. 417 p.). World Health Organization. Retrieved from http://www.cabdirect.org/abstracts/20113051318.html

Bozeat, S., Lambon Ralph, M. A., Patterson, K., Garrard, P., \& Hodges, J. R. (2000). Nonverbal semantic impairment in semantic dementia. Neuropsychologia, 38(9), 12071215. doi:10.1016/s0028-3932(00)00034-8

Breese, E. L., \& Hillis, A. E. (2004). Auditory comprehension: Is multiple choice really good enough? Brain and Language, 89(1), 3-8. doi:10.1016/s0093-934x(03)00412-7

Buchsbaum, B. R., \& D’Esposito, M. (2008). The search for the phonological store: From loop to convolution. Journal of Cognitive Neuroscience 20(5), 762-778. doi: 10.1162/jocn.2008.20501

Buchsbaum, B. R., Baldo, J., Okada, K., Berman, K. F., Dronkers, N., D’Esposito, M., \& Hickok, G. (2011). Conduction aphasia, sensory-motor integration, and phonological short-term memory - An aggregate analysis of lesion and fMRI data. Brain and Language, 119(3), 119-128. doi:10.1016/j.bandl.2010.12.001 
Buchsbaum, B. R., Olsen, R. K., Koch, P. F., Kohn, P., Kippenhan, J. S., \& Berman, K. F. (2005). Reading, hearing, and the planum temporale. NeuroImage, 24, 444-454. doi: 10.1016/j.neuroimage.2004.08.025

Buchsbaum, B. R., Olsen, R. K., Koch, P., \& Berman, K. F. (2005). Human dorsal and ventral auditory streams subserve rehearsal-based and echoic processes during verbal working memory. Neuron, 48, 687-697. doi: 10.1016/j.neuron.2005.09.029

Buiatti, T., Mussoni, A., Toraldo, A., Skrap, M., \& Shallice, T. (2011). Two qualitatively different impairments in making rotation operations. Cortex, 47(2), 166-179. doi: 10.1016/j.cortex.2009.10.006

Buiatti, T., Skrap, M., \& Shallice, T. (2012). Left- and right-hemisphere forms of phonological alexia. COGNITIVE NEUROPSYCHOLOGY, 29(7-8), 531-549. doi:10.1080/02643294.2013.771773

Bunge, S. A., Kahn, I., Wallis, J. D., Miller, E. K., \& Wagner, A. D. (2003). Neural circuits subserving the retrieval and maintenance of abstract rules. Journal of neurophysiology, 90(5), 3419-3428. doi: 10.1152/jn.00910.2002

Butterworth, B. (1992). Disorders of phonological encoding. Cognition, 42(1-3), 261-286. doi:10.1016/0010-0277(92)90045-j

Cahill, J., LoBiondo-Wood, G., Bergstrom, N., \& Armstrong, T. (2012). Brain tumor symptoms as antecedents to uncertainty: an integrative review. Journal of Nursing Scholarship : An Official Publication of Sigma Theta Tau International Honor Society of Nursing / Sigma Theta Tau, 44(2), 145-55. doi:10.1111/j.1547-5069.2012.01445.x

Cameron-Jones, C. M. L. (2008). Lexical Competition Effects in Aphasia: A Thesis Submitted to the Victoria University of Wellington in Fulfilment of the Requirements for the Degree of Doctor of Philosophy in Psychology (Doctoral dissertation, Victoria University of Wellington). 
Campanella, F., Mondani, M., Skrap, M., \& Shallice, T. (2009). Semantic access dysphasia resulting from left temporal lobe tumours. Brain: A journal of neurology, 132(1), 87102. doi: 10.1093/brain/awn302

Canter, G. J., Trost, J. E., \& Burns, M. S. (1985). Contrasting speech patterns in apraxia of speech and phonemic paraphasia. Brain and Language, 24(2), 204-222. doi:10.1016/0093-934X(85)90131-2

Caplan, D., \& Waters, G. (1999). Verbal working memory and sentence comprehension. Behavioural and Brain Sciences, 22(1), 77-94. doi:10.1017/s0140525x99001788

Caplan, D., Gow, D., \& Makris, N. (1995). Analysis of lesions by MRI in stroke patients with acoustic- phonetic processing deficits. Neurology, 45(2), 293-29. doi:10.1212/wnl.45.2.293

Caplan, D., Vanier, M., \& Baker, C. (1986). A case study of reproduction conduction aphasia I: Word production. Cognitive Neuropsychology, 3(1), 99-128. doi:10.1080/02643298608252671

Caramazza, A. (1997). How Many Levels of Processing Are There in Lexical Access? Cognitive Neuropsychology, 14(1), 177-208. doi:10.1080/026432997381664

Caramazza, A., Basili, A. G., Koller, J. J., \& Berndt, R. S. (1981). An investigation of repetition and language processing in a case of conduction aphasia. Brain and language, 14(2), 235-271. doi:10.1016/0093-934X(81)90078-X

Chang, F., Dell, G. S., \& Bock, J. K. (2006). Becoming syntactic. Psychological review, 113(2), 234. doi: 10.1037/0033-295X.113.2.234

Cloutman, L. L., Newhart, M., Davis, C. L., Heidler-Gary, J., \& Hillis, A. E. (2011). Neuroanatomical correlates of oral reading in acute left hemispheric stroke. Brain and Language, 116(1), 14-21. doi:10.1016/j.band1.2010.09.002 
Cohen, J. D., MacWhinney, B., Flatt, M., \& Provost, J. (1993). PsyScope: A new graphic interactive environment for designing psychology experiments. Behavioral Research Methods, Instruments, and Computers, 25(2), 257-271. doi:10.3758/bf03204507

Cole, R. A., \& Jakimik, J. (1980). A model of speech perception. In R. Cole (Ed.), Perception and production of fluent speech. Hillsdale, NJ: Erlbaum.

Collins, A. M., \& Loftus, E. F. (1975). A spreading-activation theory of semantic processing. Psychological review, 82(6), 407. doi:10.1016/b978-1-4832-1446-7.50015-7

Coltheart, M. (1985). In defence of dual-route models of reading. Behavioral and Brain Sciences, 8(04), 709-710. doi:10.1017/s0140525x0004574x

Coltheart, M., \& Rastle, K. (1994). Serial processing in reading aloud: Evidence for dualroute models of reading. Journal of Experimental Psychology: Human Perception and Performance. doi:10.1037/0096-1523.20.6.1197

Coltheart, M., Curtis, B., Atkins, P., \& Haller, M. (1993). Models of reading aloud: Dualroute and parallel-distributed-processing approaches. Psychological review, 100(4), 589. oi:10.1037/0033-295x.100.4.589

Coltheart, M., Rastle, K., Perry, C., Langdon, R., \& Ziegler, J. (2001). DRC: a dual route cascaded model of visual word recognition and reading aloud. Psychological Review, 108(1), 204-256. doi:10.1037/0033-295X.108.1.204

Corbett, F., Jefferies, E., Ehsan, S., \& Lambon Ralph, M. A. (2009). Different impairments of semantic cognition in semantic dementia and semantic aphasia: evidence from the non-verbal domain. Brain : A Journal of Neurology, 132(9), 2593-2608. doi:10.1093/brain/awp146

Crawford, J. R., Howell, D. C., \& Garthwaite, P. H. (1998). Payne and Jones Revisited: Estimating the Abnormality of Test Score Differences Using a Modified Paired 
Samples t Test. Journal of Clinical and Experimental Neuropsychology, 20(6), 898905. doi:10.1076/jcen.20.6.898.1112

Crosson, B., Sadek, J. R., Maron, L., Gökçay, D., Mohr, C. M., Auerbach, E. J., Freeman, A. J., et al. (2001). Relative shift in activity from medial to lateral frontal cortex during internally versus externally guided word generation. Journal of cognitive neuroscience, 13(2), 272-283. doi: 10.1162/089892901564225

Dahan, D., Magnuson, J. S., Tanenhaus, M. K., \& Hogan, E. M. (2001). Subcategorical mismatches and the time course of lexical access: Evidence for lexical competition. Language and Cognitive Processes. doi:10.1080/01690960143000074

Damasio, H., Grabowski, T. J., Tranel, D., Hichwa, R. D., \& Damasio, A. R. (1996). A neural basis for lexical retrieval. Nature, 381(6585), 810-810. Doi: 10.1038/381810b0

Damasio, H., Tranel, D., Grabowski, T., Adolphs, R., \& Damasio, A. (2004). Neural systems behind word and concept retrieval. Cognition, 92(1-2), 179-229. doi:10.1016/j.cognition.2002.07.001

Darley, F. L. (1982). Aphasia. New York: Saunders.

Darley, F. L., Aronson, A. E. \& Brown, J. R. (1975) Motor Speech Disorders Saunders, Philadelphia, 1975.

Davidson, P. S. R., Gao, F. Q., Mason, W. P., Winocur, G., \& Anderson, N. D. (2008). Verbal fluency, trail making, and Wisconsin Card Sorting Test performance following right frontal lobe tumor resection. Journal of clinical and experimental neuropsychology, 30(1), 18-32. doi:10.1080/13803390601161166

Davie, G. L., Hutcheson, K. A., Barringer, D. A., Weinberg, J. S., \& Lewin, J. S. (2009). Aphasia in patients after brain tumour resection. Aphasiology, 23(9), 1196-1206. doi:10.1080/02687030802436900 
Davies, E., \& Clarke, C. (2004). Early symptoms of brain tumours. Journal of Neurology, Neurosurgery \& Psychiatry, 75(8), 1205-1206. doi:10.1136/jnnp.2003.033308

De Benedictis, A., Moritz-Gasser, S., \& Duffau, H. (2010). Awake mapping optimizes the extent of resection for low-grade gliomas in eloquent areas. Neurosurgery, 66(6), 1074-1084; discussion 1084. doi: 10.1227/01.NEU.0000369514.74284.78.

De Witte, E., \& Mariën, P. (2013). The neurolinguistic approach to awake surgery reviewed. Clinical neurology and neurosurgery, 115(2), 127-145. doi:10.1016/j.clineuro.2012.09.015

DeAngelis, L. M. (2001). Brain tumors. New England Journal of Medicine, 344(2), 114-123. . Retrieved from http://www.nejm.org/doi/full/10.1056/NEJM200101113440207

Dehaene, S., \& Cohen, L. (2011). The unique role of the visual word form area in reading. Trends in cognitive sciences, 15(6), 254-262. doi:10.1016/j.tics.2011.04.003

DeLeon, J., Gottesman, R. F., Kleinman, J. T., Newhart, M., Davis, C., Heidler-Gary, J., ... Hillis, A. E. (2007). Neural regions essential for distinct cognitive processes underlying picture naming. Brain: A Journal of Neurology, 130(5), 1408-1422. doi:10.1093/brain/awm011

Dell, G. S. (1986). A spreading-activation theory of retrieval in sentence production. Psychological Review, 93(3), 283-321. doi:10.1037/0033-295x.93.3.283

Dell, G. S., Lawler, E. N., Harris, H. D., \& Gordon, J. K. (2004). Models of errors of omission in aphasic naming. Cognitive Neuropsychology, 21(2), 125-145. doi:10.1080/02643290342000320

Dell, G. S., Schwartz, M. F., Martin, N., Saffran, E. M., \& Gagnon, D. A. (1997). Lexical access in aphasic and nonaphasic speakers. Psychological review, 104(4), 801. doi:10.1037/0033-295x.104.4.801 
Dell, G. S., Schwartz, M. F., Nozari, N., Faseyitan, O., \& Branch Coslett, H. (2013). Voxelbased lesion-parameter mapping: Identifying the neural correlates of a computational model of word production. Cognition, 128(3), 380-396.

doi:10.1016/j.cognition.2013.05.007

DeMarco, A., Rising, K., Wilson, S. M., Rapcsak, S. Z. \& Beeson, P. M. (2012, October 28). The neural substrates of improved phonological processing following successful treatment in a case of phonological alexia and agraphia. Presented at the $50^{\text {th }}$ Annual Meeting of the Academy of Aphasia, San Francisco, CA.

Dronkers, N. F. (1996). A new brain region for coordinating speech articulation. Nature, 384(6605), 159-161. doi:10.1038/384159a0

Duffau, H. (2007). Contribution of cortical and subcortical electrostimulation in brain glioma surgery: Methodological and functional considerations. Neurophysiologie Clinique, 37(6), 373-382. doi: 10.1016/j.neucli.2007.09.003

Duffau, H. (2007). Contribution of cortical and subcortical electrostimulation in brain glioma surgery: methodological and functional considerations. Neurophysiologie Clinique/Clinical Neurophysiology, 37(6), 373-382. doi:10.1016/j.neucli.2007.09.003

Duffau, H., Peggy Gatignol, S. T., Mandonnet, E., Capelle, L., \& Taillandier, L. (2008). Intraoperative subcortical stimulation mapping of language pathways in a consecutive series of 115 patients with Grade II glioma in the left dominant hemisphere. Journal of Neurosurgery, 109(3), 461-471. doi:10.3171/jns/2008/109/9/0461

Ellis, A. W. (1980). Errors in speech and short-term memory: The effects of phonemic similarity and syllable position. Journal of Verbal Learning and Verbal Behavior. doi:10.1016/S0022-5371(80)90672-6

Farah, M. J., \& Wallace, M. A. (1991). Pure Alexia as a Visual Impairment: A Reconsideration. Cognitive Neuropsychology. doi:10.1080/02643299108253376 
Finch, E. \& Copland D. A. (2014). Language outcomes following neurosurgery for brain tumours: A systematic review. NeuroRehabilitation, 34, 499-514. doi: 10.3233/NRE141053

Forsyth, P. A., \& Posner, J. B. (1993). Headaches in patients with brain tumors: A study of 111 patients. Neurology, 43(9), 1678-1678. doi:10.1212/wnl.43.9.1678

Foundas, A. L. (2001). The Anatomical Basis of Language. Topics in Language Disorders. 21(3), 1-19. doi:10.1097/00011363-200121030-00003

Franklin, S. (1989). Dissociations in auditory word comprehension; evidence from nine fluent aphasic patients. Aphasiology, 3(3), 189-207. doi: $10.1080 / 02687038908248991$

Franklin, S., Howard, D., \& Patterson, K. (1995). Abstract word anomia. Cognitive Neuropsychology, 12(5), 549-566. doi:10.1080/02643299508252007

Frey, R. T., Woods, D. L., Knight, R. T., Scabini, D., \& Clayworth, C. (1987). Defining functional areas with averaged CT scans. In Society for Neuroscience Abstracts (Vol. 13 , p. 1266).

Friederici, A. D. (2014). White-matter pathways for speech and language processing. Handbook of clinical neurology, 129, 177-186.

Friedmann, N., Biran, M., \& Dotan, D. (2013). Lexical retrieval and breakdown in aphasia and developmental language impairment. The Cambridge handbook of biolinguistics, $350-374$.

Funnell, E. (1983). Phonological processes in reading: New evidence from acquired dyslexia. British Journal of Psychology, 74(2), 159-180. doi:10.1093/neucas/1.3.251-g

Garrard, P., Perry, R., \& Hodges, J. R. (1997). Disorders of semantic memory. Journal of neurology, neurosurgery, and psychiatry, 62(5), 431. Retrieved from http://www.ncbi.nlm.nih.gov/pmc/articles/PMC486839/ 
Garrett, M. F. (1975). The analysis of sentence production. Psychology of learning and motivation, 9, 133-177. doi:10.1016/s0079-7421(08)60270-4

Garrett, M. F. (1976). Syntactic processes in sentence production. In R. J. Wales \& E. Walker (Eds.), New approaches to language mechanisms, (pp. 231-256). Amsterdam: NorthHolland Linguistic Series, 30.

Garrett, M. F. (1980). Levels of processing in sentence production. In B. Butterworth (Ed.), Language production (pp. 177-220). London: Academic Press.

Gathercole, S. E., \& Baddeley, A. D. (1989). Evaluation of the role of phonological STM in the development of vocabulary in children: A longitudinal study. Journal of Memory and Language, 28(2), 200-213. doi:10.1016/0749-596X(89)90044-2

Gathercole, S. E., Willis, C. S., Emslie, H., \& Baddeley, A. D. (1992). Phonological memory and vocabulary development during the early school years: A longitudinal study. Developmental Psychology. doi:10.1037/0012-1649.28.5.887

Gladsjo, J. A., Miller, S. W., \& Heaton, R. K. (1999). Norms for Letter and Category Fluency: Demographic Corrections for Age, Education, and Ethnicity. Psychological Assessment Resources, Inc, Odessa.

Glaser, W. R., \& Düngelhoff, F. J. (1984). The time course of picture-word interference. Journal of Experimental Psychology: Human Perception and Performance, 10(5), 640-654. doi:10.1037/0096-1523.10.5.640

Gonon, M. A., Bruckert, R., \& Michel, F. (1989). Lexicalization in an anomic patient. Neuropsychologia, 27(4), 391-407. doi:10.1016/0028-3932(89)90047-x

Goodglass, H., \& Kaplan, E. (1983). Boston diagnostic aphasia examination booklet. Lea \& Febiger.

Goodglass, H., Kaplan, E., \& Barresi, B. (2001). The assessment of aphasia and related disorders. Lippincott Williams \& Wilkins. 
Graham, K. S., \& Hodges, J. R. (1997). Differentiating the roles of the hippocampal complex and the neocortex in long-term memory storage: evidence from the study of semantic dementia and Alzheimer's disease. Neuropsychology, 11(1), 77-89. doi 10.1037//0894-4105.11.1.77

Gupta, D. K., Chandra, P. S., Ojha, B. K., Sharma, B. S., Mahapatra, A. K., \& Mehta, V. S. (2007). Awake craniotomy versus surgery under general anesthesia for resection of intrinsic lesions of eloquent cortex-A prospective randomised study. Clinical Neurology and Neurosurgery, 109(4), 335-343. doi:10.1016/j.clineuro.2007.01.008

Gupta, P. (2003). Examining the relationship between word learning, nonword repetition, and immediate serial recall in adults. The Quarterly Journal of Experimental Psychology. A, Human Experimental Psychology, 56(7), 1213-1236.

doi:10.1080/02724980343000071

Gupta, P., MacWhinney, B., Feldman, H. M., \& Sacco, K. (2003). Phonological memory and vocabulary learning in children with focal lesions. Brain and Language, 87(2), 241252. doi:10.1016/S0093-934X(03)00094-4

Gutin, P. H., \& Posner, J. B. (2000). Neuro-oncology: Diagnosis and management of cerebral gliomas-past, present, and future. Neurosurgery, 47(1), 1-8. doi:10.1097/00006123200007000-00001

Gvion, A., \& Friedmann, N. (2012). Does phonological working memory impairment affect sentence comprehension? A study of conduction aphasia. Aphasiology. doi:10.1080/02687038.2011.647893

Haglund, M. M., Berger, M. S., Shamseldin, M., Lettich, E., \& Ojemann, G. A. (1994). Cortical localization of temporal lobe language sites in patients with gliomas. Neurosurgery, 34(4), 567-576. doi:10.1097/00006123-199404000-00001 
Hamilton, A. C., \& Martin, R. C. (2005). Dissociations among tasks involving inhibition: A single-case study. Cognitive, Affective, \& Behavioral Neuroscience, 5(1), 1-13. doi:10.3758/CABN.5.1.1

Hanley, J. R., \& Kay, J. (1996). Reading speed in pure alexia. Neuropsychologia, 34(12), 1165-1174. doi:10.1016/0028-3932(96)00039-5

Harm, M. W., \& Seidenberg, M. S. (1999). Phonology, reading acquisition, and dyslexia: insights from connectionist models. Psychological Review, 106(3), 491-528. doi:10.1037/0033-295X.106.3.491

Harm, M. W., \& Seidenberg, M. S. (2004). Computing the meanings of words in reading: cooperative division of labour between visual and phonological processes. Psychological Review, 111(3), 662-720. doi:10.1037/0033-295x.111.3.662

Heimans, J. J., \& Reijneveld, J. C. (2012). Factors affecting the cerebral network in brain tumor patients. Journal of neuro-oncology, 108(2), 231-237. doi:10.1007/s11060012-0814-7

Henry, J. D., \& Crawford, J. R. (2004). A meta-analytic review of verbal fluency performance following focal cortical lesions. Neuropsychology, 18(2), 284-295. doi:10.1037/0894-4105.18.2.284

Henry, M. L., Beeson, P. M., Stark, A. J., \& Rapcsak, S. Z. (2007). The role of left perisylvian cortical regions in spelling. Brain and Language, 100(1), 44-52. doi:10.1016/j.band1.2006.06.011

Henseler, I., Regenbrecht, F., \& Obrig, H. (2014). Lesion correlates of patholinguistic profiles in chronic aphasia: comparisons of syndrome-, modality- and symptom-level assessment. Brain, 137(3), 918-930. doi:10.1093/brain/awt374

Hickok, G., \& Poeppel, D. (2007). Opinion - The cortical organization of speech processing. Nature Reviews Neuroscience, 8(5), 393-402. doi:10.1038/nrn2113 
Hillis, A. E., Kleinman, J. T., Newhart, M., Heidler-Gary, J., Gottesman, R., Barker, P. B., Aldrich, E., et al. (2006). Restoring cerebral blood flow reveals neural regions critical for naming. Journal of Neuroscience, 26(31), 8069-8073. doi: 10.1523/jneurosci.2088-06.2006

Hodges, J. R., \& Patterson, K. (2007). Semantic dementia: a unique clinicopathological syndrome. The Lancet Neurology, 6(11), 1004-1014. doi:10.1016/s14744422(07)70266-1

Hodges, J. R., Graham, N., \& Patterson, K. (1995). Charting the progression in semantic dementia: Implications for the organization of semantic memory. Memory, 3(3), 463495. doi:10.1080/09658219508253161

Hodges, J. R., Patterson, K., Oxbury, S., \& Funnell, E. (1992). Semantic dementia progressive fluent aphasia with temporal lobe atrophy. Brain, 115(6), 1783-1806. doi:10.1093/brain/115.6.1783

Howard, D. (1995). Lexical anomia: Or the case of the missing lexical entries. The Quarterly Journal of Experimental Psychology, 48(4), 999-1023.. doi:10.1080/14640749508401426

Huber, W., Poeck, K., \& Willmes, K. (1983). The Aachen Aphasia Test. Advances in neurology, 42, 291-303.

Ilmberger, J., Ruge, M., Kreth, F. W., Briegel, J., Reulen, H. J., \& Tonn, J. C. (2008). Intraoperative mapping of language functions: a longitudinal neurolinguistic analysis. Journal of Neurosurgery, 109(4), 583-592. doi:10.3171/jns/2008/109/10/0583

Indefrey, P., \& Levelt, W. J. M. (2004). The spatial and temporal signatures of word production components. Cognition, 92(1-2), 101-144. doi:10.1016/j.cognition.2002.06.001 
Jacobs, M., Singer, N., \& Miozzo, M. (2004). The representation of homophones: Evidence from anomia. Cognitive Neuropsychology, 21(8), 840-866. doi:

10.1162/jocn.2008.21179

January, D., Trueswell, J. C., \& Thompson-Schill, S. L. (2009). Co-localization of stroop and syntactic ambiguity resolution in Broca's area: implications for the neural basis of sentence processing. Journal of Cognitive Neuroscience, 21(12), 2434-2444.

Jefferies, E., \& Lambon Ralph, M. A. (2006). Semantic impairment in stroke aphasia versus semantic dementia: a case-series comparison. Brain : A Journal of Neurology, 129(8), 2132-2147. doi:10.1093/brain/awl153

Jellison, B. J., Field, A. S., Medow, J., Lazar, M., Salamat, M. S., \& Alexander, A. L. (2004). Diffusion tensor imaging of cerebral white matter: a pictorial review of physics, fiber tract anatomy, and tumor imaging patterns. American Journal of Neuroradiology, 25(3), 356-369. doi: 10.1016/S0030-6657(09)70151- 9

Jobard, G., Crivello, F., \& Tzourio-Mazoyer, N. (2003). Evaluation of the dual route theory of reading: A metanalysis of 35 neuroimaging studies. NeuroImage, 20(2), 693-712. doi:10.1016/s1053-8119(03)00343-4

Johns, D. F., \& Darley, F. L. (1970). Phonemic variability in apraxia of speech. Journal of Speech, Language, and Hearing Research, 13(3), 556-583. doi:10.1044/jshr.1303.556

Kauhanen, M. L., Korpelainen, J. T., Hiltunen, P., Määttä, R., Mononen, H., Brusin, E., ... Myllylä, V. V. (2000). Aphasia, depression, and non-verbal cognitive impairment in ischaemic stroke. Cerebrovascular Diseases Basel Switzerland, 10(6), 455-461. doi:10.1159/000016107

Kay, J., Lesser, R., \& Coltheart, M. (1996). Psycholinguistic assessments of language processing in aphasia (PALPA): An introduction. Aphasiology, 10(2), 159-180. doi:10.1080/02687039608248403 
Kent, R. D., \& Rosenbek, J. C. (1983). Acoustic patterns of apraxia of speech. Journal of Speech, Language, and Hearing Research, 26(2), 231-249. doi:10.1044/jshr.2602.231

Kertesz, A. (1982). Western aphasia battery test manual. Psychological Corp.

Kertesz, A., \& Sheppard, A. (1981). The epidemiology of aphasic and cognitive impairment in stroke: age, sex, aphasia type and laterality differences. Brain: A Journal of Neurology, 104(1), 117-128. doi:10.1093/brain/104.1.117

Kerzel, D., \& Bekkering, H. (2000). Motor activation from visible speech: evidence from stimulus response compatibility. Journal of Experimental Psychology. Human Perception and Performance, 26(2), 634-647. doi:10.1037/0096-1523.26.2.634

Kimberg, D. Y., Coslett, H. B., \& Schwartz, M. F. (2007). Power in Voxel-based lesionsymptom mapping. Journal of cognitive neuroscience, 19(7), 1067-1080. doi: 10.1162/jocn.2007.19.7.1067

Kleihues, P., \& Sobin, L. H. (2000). World Health Organization classification of tumors. Cancer, 88(12), 2887-2887. doi: 10.1002/1097-0142(20000615)88:12<2887::AIDCNCR32>3.0.CO;2-F

Klein, M., Heimans, J. J., Aaronson, N. K., Van Der Ploeg, H. M., Grit, J., Muller, M., \& Taphoorn, M. J. B. (2002). Effect of radiotherapy and other treatment-related factors on mid-term to long-term cognitive sequelae in low-grade gliomas: a comparative study. Lance, 360(9343), 1361-1368. doi:10.1016/s0140-6736(02)11398-5

Kling, T. D. (2007). A voxel-based approach to identifying lesion sites in aphasia: Comprehension and production deficits in syntax, semantics and phonology (Unpublished doctorial dissertation). University of Mayland.

Kohn, S. E., \& Goodglass, H. (1985). Picture-naming in aphasia. Brain and Language, 24(2), 266-283. doi:10.1016/0093-934X(85)90135-X 
Kohn, S. E., \& Smith, K. L. (1993). Lexical-phonological processing of functors: Evidence from fluent aphasia. Cortex, 29(1), 53-64. doi:10.1016/s0010-9452(13)80211-0

Kucera, F. WN, 1967. Computational Analysis of Present-Day American English. Providence Brown UP.

Laine, M., Kujala, P., Niemi, J., \& Uusipaikka, E. (1992). On the nature of naming difficulties in aphasia. Cortex; a Journal Devoted to the Study of the Nervous System and Behavior, 28(4), 537-554. doi:10.1016/S0010-9452(13)80226-2

Lambon Ralph, M. A., Sage, K., \& Roberts, J. (2000). Classical anomia: A neuropsychological perspective on speech production. Neuropsychologia, 38(2), 186202. doi:10.1016/s0028-3932(99)00056-1

Levelt, W. (1999). Models of word production. Trends in Cognitive Sciences, 3(6), 223-232. doi:10.1016/S1364-6613(99)01319-4

Levelt, W. J. (1992). Accessing words in speech production: Stages, processes and representations. Cognition, 42(1), 1-22. doi:10.1016/0010-0277(92)90038-j

Levelt, W. J. M. (1989) Speaking: From intention to articulation. Bradford, Cambridge, MA.

Levelt, W. J., \& Wheeldon, L. (1994). Do speakers have access to a mental syllabary? Cognition, 50(1), 239-269. doi:10.1016/0010-0277(94)90030-2

Lezak, M. D. (1982). Neuropsychological Assessment, $2^{\text {nd }}$ Edition. Oxford University Press. MacLeod, C. M. (1991). Half a century of research on the Stroop effect: an integrative review. Psychological Bulletin, 109(2), 163-203. doi:10.1037/0033-2909.109.2.163

MacPherson, S. E., Turner, M. S., Bozzali, M., Cipolotti, L., \& Shallice, T. (2010). Frontal subregions mediating Elevator Counting task performance.

Neuropsychologia, 48(12), 3679-3682. doi: 10.1016/j.neuropsychologia.2010.07.033

Marsh, H. (2009). Brain tumours. Surgery, 27(3), 135-138. Retrieved from http://www.surgeryjournal.co.uk/article/S0263-9319(09)00054-4/abstract 
Marshall, J., Pring, T., \& Chiat, S. (1998). Verb retrieval and sentence production in aphasia. Brain and Language, 63(2), 159-183. doi:10.1006/brln.1998.1949

Marslen-Wilson, W. D. (1987). Functional parallelism in spoken word-recognition. Cognition, 25(1), 71-102. doi:10.1016/0010-0277(87)90005-9

Marslen-Wilson, W. D., \& Welsh, A. (1978). Processing interactions and lexical access during word recognition in continuous speech. Cognitive Psychology, 10(1), 29-63. doi:10.1016/0010-0285(78)90018-X

Marslen-Wilson, W., \& Tyler, L. K. (1980). The temporal structure of spoken language understanding. Cognition, 8(1), 1-71. doi:10.1016/0010-0277(80)90015-3

Martin, N., \& Gupta, P. (2004). Exploring the relationship between word processing and verbal short term memory: Evidence from association and dissociation. Cognitive Neuropsychology, 21(2-4), 213-228. doi: 10.1080/02643290342000447

Martin, N., \& Saffran, E. M. (1997). Language and auditory-verbal short-term memory impairments: Evidence for common underlying processes. Cognitive Neuropsychology, 14, 641-682. doi: 10.1080/026432997381402

Martin, N., Schwartz, M., \& Kohen, F. P. (2006). Assessment of the ability to process semantic and phonological aspects of words in aphasia: A multi-measurement approach. Aphasiology, 20(2-4), 154-166. doi:10.1080/02687030500472520

Martin, R. C., \& Cheng, Y. (2006). Selection demands versus association strength in the verb generation task. Psychonomic Bulletin Review, 13(3), 396-401. doi:10.3758/bf03193859

Martin, R. C., \& Feher, E. (1990). The consequences of reduced memory span for the comprehension of semantic versus syntactic information. Brain and Language, 38(1), 1-20. oi:10.1016/0093-934x(90)90099-3 
Martin, R. C., \& Romani, C. (1994). Verbal working memory and sentence comprehension: A multiple-components view. Neuropsychology. doi:10.1037/0894-4105.8.4.506

Masson, M. E. (1991). A distributed memory mode of contact effects in word identification In D. Besner \& G. W. Humphreys (Eds.), Basic processes in reading: Visual word recognition, 233-263. Hillsdale, NJ: Erlbaum.

Masson, M. E. (1995). A distributed memory model of semantic priming. Journal of Experimental Psychology: Learning, Memory, and Cognition, 21(1), 3. doi:10.1037/0278-7393.21.1.3

Mätzig, S., Druks, J., Masterson, J., \& Vigliocco, G. (2009). Noun and verb differences in picture naming: Past studies and new evidence. Cortex, 45(6), 738-758. doi:10.1016/j.cortex.2008.10.003

McCarthy, R. A., \& Kartsounis, L. D. (2000). Wobbly words: Refractory anomia with preserved semantics. Neurocase, 6(6), 487-497. doi:10.1093/neucas/6.6.497

McCarthy, R., \& Warrington, E. K. (1984). A two-route model of speech production. Brain, Mcclelland, J. L., Elman, J. L., \& Diego, S. (1986). The TRACE Model of Speech Perception. Cognitive Psychology, 18(1), 1-86. doi:10.1016/0010-0285(86)90015-0

McGirt, M. J., Mukherjee, D., Chaichana, K. L., Than, K. D., Weingart, J. D., \& QuinonesHinojosa, A. (2009). Association of surgically acquired motor and language deficits on overall survival after resection of glioblastoma multiforme. Neurosurgery, 65(3), 463-469. doi:10.1227/01.neu.0000349763.42238.e

McNeil, M. R., Odell, K., \& Tseng, C. H. (1991). Toward the integration of resource allocation into a general theory of aphasia. Clinical Aphasiology, 20(21-39). Retrieved from http://aphasiology.pitt.edu/archive/00000128/

Medina, J., Kimberg, D. Y., Chatterjee, A., \& Coslett, H. (2010). Inappropriate usage of the Brunner-Munzel test in recent voxel-based lesion-symptom mapping 
studies. Neuropsychologia, 48(1), 341-343.doi:

10.1016/j.neuropsychologia.2009.09.016

Meyers, C. A., \& Brown, P. D. (2006). Role and relevance of neurocognitive assessment in clinical trials of patients with CNS tumours. J Clin Oncol, 24(8), 1305-1309. doi:10.1200/jco.2005.04.6086

Meyers, C. A., Hess, K. R., Yung, W. A., \& Levin, V. A. (2000). Cognitive function as a predictor of survival in patients with recurrent malignant glioma. Journal of Clinical Oncology, 18(3), 646-646. Retrieved fro, http://jco.ascopubs.org/content/18/3/646.short

Miceli, G., Amitrano, A., Capasso, R., \& Caramazza, A. (1996). The treatment of anomia resulting from output lexical damage: Analysis of two cases. Brain and Language, 52(1), 150-174. doi:10.1006/brln.1996.0008

Miceli, G., Capasso, R., Monti, A., Santini, B., \& Talacchi, A. (2012). Language testing in brain tumor patients. Journal of neuro-oncology, 108(2), 247252.doi:10.1007/s11060-012-0810-y

Miceli, G., Gainotti, G., Caltagirone, C., \& Masullo, C. (1980). Some aspects of phonological impairment in aphasia. Brain and Language, 11(1), 159-169. doi:10.1016/0093$934 \mathrm{X}(80) 90117-0$

Miller, L. S., \& Rohling, M. L. (2001). A statistical interpretive method for neuropsychological test data. Neuropsychology Review, 11(3), 143-169. doi: 10.1023/A:1016602708066

Mirman, D., \& Graziano, K. M. (2013). The Neural Basis of Inhibitory Effects of Semantic and Phonological Neighbors in Spoken Word Production. Journal of Cognitive Neuroscience, 25(9), 1504-1516. doi:10.1162/jocn 
Mitrushina, M. N., Boone, K B., \& D’Elia (1999). Handbook of normative data for neuropsychological assessment. New York: Oxford University Press.

Moritz-Gasser, S., Herbet, G., Maldonado, I. L., \& Duffau, H. (2012). Lexical access speed is significantly correlated with the return to professional activities after awake surgery for low-grade gliomas. Journal of Neurooncology, 107(3), 633-41. doi:10.1007/s11060-011-0789-9

Mummery, C. J., Patterson, K., Price, C. J., Ashburner, J., Frackowiak, R. S. J., \& Hodges, J. R. (2000). A voxel-based morphometry study of semantic dementia: relationship between temporal lobe atrophy and semantic memory. Annals of neurology, 47(1), 36-45. doi: 10.1002/1531-8249(200001)47:1<36::aid-ana8>3.3.co;2-c

Murray, K. J., Scott, C., Zachariah, B., Michalski, J. M., Demas, W., Vora, N. L., \& Movsas, B. (2000). Importance of the mini-mental status examination in the treatment of patients with brain metastases: a report from the Radiation Therapy Oncology Group protocol 91-04. International Journal of Radiation Oncology, Biology, Physics, 48(3), 59-64. doi:10.1016/s0360-3016(00)00600-3

Myachykov, A. \& Posner M. I. (2005). “Attention in Language”. In Itti, L., Rees, G., \& Tsotsos, J. K. (Eds.) Neurobiology of attention. Academic Press. 324-329.

Neely, J. H. (1997). Semantic priming and retrieval from lexical memory: Roles of inhibition less spreading activation and limited-capacity attention. Journal of experimental psychology: general 106(3) 226. doi:10.3758/bf03213230

Nickels, L. (2002). Theoretical and methodological issues in the cognitive neuropsychology of spoken word production. Aphasiology, 16(1-2), 3-19. doi:10.1080/02687040143000645

Nickels, L., \& Howard, D. (2004). Dissociating Effects of Number of Phonemes, Number of Syllables, and Syllabic Complexity on Word Production in Aphasia: It's the Number 
of Phonemes that Counts. Cognitive Neuropsychology, 21(1), 57-78.

doi:10.1080/02643290442000221

Norris, D., McQueen, J. M., \& Cutler, A. (2000). Merging information in speech recognition: feedback is never necessary. Behavioral and Brain Sciences, 23(3), 299-325. doi:10.1017/s0140525x00003241

Novick, J. M., Kan, I. P., Trueswell, J. C., \& Thompson-Schill, S. L. (2009). A case for conflict across multiple domains: memory and language impairments following damage to ventrolateral prefrontal cortex. Cognitive Neuropsychology, 26(6), 527567. doi: $10.1080 / 02643290903519367$

Novick, J. M., Trueswell, J. C., \& Thompson-Schill, S. L. (2005). Cognitive control and parsing: Reexamining the role of Broca's area in sentence comprehension. Cognitive, Affective, \& Behavioral Neuroscience, 5(3), 263-281. doi: 10.3758/cabn.5.3.263

Novick, J. M., Trueswell, J. C., \& Thompson- Schill, S. L. (2010). Broca’s area and language processing: Evidence for the cognitive control connection. Language and Linguistics Compass, 4(10), 906-924. doi: 10.1111/j.1749-818x.2010.00244.x

Ogar, J. M., Dronkers, N. F., Brambati, S. M., Miller, B. L., \& Gorno-Tempini, M. L. (2007). Progressive Nonfluent Aphasia and Its Characteristic Motor Speech Deficits. Alzheimer Disease \& Associated Disorders, 21(4), S23-S30. doi:10.1097/wad.0b013e31815d19fe

Ogar, J., \& Gorno-Tempini, M. L. (2009). Progressive non-fluent aphasia. The Behavioral Neurology of Dementia, 279-287. doi:10.1017/cbo9780511581410.019

Ogar, J., Slama, H., Dronkers, N., Amici, S., \& Luisa Gorno-Tempini, M. (2005). Apraxia of speech: an overview. Neurocase, 11(6), 427-432. doi:10.1080/13554790500263529 
Omuro, A. M., Leite, C. C., Mokhtari, K., \& Delattre, J.-Y. (2006). Pitfalls in the diagnosis of brain tumours. Lancet Neurology, 5(11), 937-948. doi:10.1016/s14744422(06)70597-x

Påhlson, A., Ek, L., Ahlström, G., \& Smits, A. (2003). Pitfalls in the assessment of disability in individuals with low-grade gliomas. Journal of Neuro-Oncology, 65(2), 149-158. doi:10.1023/b:neon.0000003727.09448.dd

Papagno, C., Casarotti, A., Comi, A., Gallucci, M., Riva, M., \& Bello, L. (2012). Measuring clinical outcomes in neuro-oncology. A battery to evaluate low-grade gliomas (LGG). J Neurooncol, 108, 269-275. doi:10.1007/s11060-012-0824-5

Park, B.J, Kim, H. K., Sade, B., Lee, J. H. (2009). “Epidemiology”. In Lee J. H. Meningiomas: Diagnosis, Treatment and Outcome. Springer. p. 11. ISBN 978-184882-910-7.

Pashek, G. V, \& Holland, A. L. (1988). Evolution of aphasia in the first year post-onset. Cortex, 24(3), 411-423. doi:10.1016/s0010-9452(88)80004-2

Pate, D. S., Saffran, E. M., \& Martin, N. (1987). Specifying the nature of the production impairment in a conduction aphasic: A case study. Language and Cognitive Processes, 2(1), 43-84. doi:10.1080/01690968708406351

Patterson, K., \& Shewell, C. (1987). Speak and spell: Dissociations and word-class effects. In M. G. Coltheart, G. Sartori, \& R. Job (Eds.), The cognitive neuropsychology of language (273-294). Erlbaum.

Pendleton, M. G., Heaton, R. K., Lehman, R. A., \& Hulihan, D. (1982). Diagnostic utility of the Thurstone Word Fluency Test in neuropsychological evaluations. Journal of Clinical Neuropsychology, 4(4), 307-317. doi:10.1080/01688638208401139 
Perret, E. (1974). The left frontal lobe of man and the suppression of habitual responses in verbal categorical behaviour. Neuropsychologia, 12(3), 323-330. doi:10.1016/00283932(74)90047-5

Perry, C., Ziegler, J. C., \& Zorzi, M. (2007). Nested incremental modeling in the development of computational theories: the CDP + model of reading aloud. Psychological review, 114(2), 273. doi:10.1037/0033-295x.114.2.273

Peruzzi, P., Bergese, S. D., Viloria, A., Puente, E. G., Abdel-Rasoul, M., \& Chiocca, E. A. (2011). A retrospective cohort-matched comparison of conscious sedation versus general anesthesia for supratentorial glioma resection. Clinical article. Journal of Neurosurgery, 114(3), 633-639. doi:10.3171/2010.5.JNS1041

Pettorini, B. L., Park, Y.-S., Caldarelli, M., Massimi, L., Tamburrini, G., \& Di Rocco, C. (2008). Radiation-induced brain tumours after central nervous system irradiation in childhood: a review. Child's nervous system : ChNS : official journal of the International Society for Pediatric Neurosurgery 793-805 doi:10.1007/s00381-008$0631-7$

Pijnenburg, Y. A. L., Gillissen, F., Jonker, C., \& Scheltens, P. (2004). Initial complaints in frontotemporal lobar degeneration. Dementia and Geriatric Cognitive Disorders, 17(4), 302-306. doi:10.1159/000077159

Piras, F., \& Marangolo, P. (2007). Noun-verb naming in aphasia: a voxel-based lesionsymptom mapping study. Neuroreport, 18(14), 1455-1458.

doi:10.1097/WNR.0b013e3282ef6fc9

Piras, F., \& Marangolo, P. (2010). When "Crack walnuts” lies in different brain regions: evidence from a voxel-based lesion-symptom mapping study. Journal of the International Neuropsychological Society : JINS, 16(3), 433-442. doi:10.1017/S1355617710000068 
Plaut, D. C., McClelland, J. L., Seidenberg, M. S., \& Patterson, K. (1996). Understanding normal and impaired word reading: computational principles in quasi-regular domains. Psychological Review, 103(1), 56-115. doi:10.1037/0033-295X.103.1.56

Plaza, M., Gatignol, P., Leroy, M., \& Duffau, H. (2009). Speaking without Broca's area after tumor resection. Neurocase, 15(4), 294-310. doi: 10.1080/13554790902729473

Porter, G., \& Howard, D. (2004). CAT: comprehensive aphasia test. Psychology Press.

Porter, K. R., McCarthy, B. J., Freels, S., Kim, Y., \& Davis, F. G. (2010). Prevalence estimates for primary brain tumors in the United States by age, gender, behavior, and histology. Neuro-Oncology, 12(6), 520-527. doi: 10.1093/neuonc/nop066

Pradat-Diehl, P., Tessier, C., Vallat, C., Mailhan, L., Mazevet, D., Lauriot-Prevost, M. C., \& Bergego, C. (2001). [Conduction aphasia and phonemic disorder]. Revue neurologique, 157(10), 1245-1252. Retrieved from http://europepmc.org/abstract/med/11885517

Rapp, B., \& Goldrick, M. (2000). Discreteness and interactivity in spoken word production. Psychological review, 107(3), 460. doi=10.1037/0033-295X.107.3.460

Rapp, B., \& Goldrick, M. (2000). Discreteness and interactivity in spoken word production. Psychological review, 107(3), 460. doi:10.1037/0894-4105.21.1.20

Rascovsky, K., Salmon, D. P., Hansen, L. A., Thal, L. J., \& Galasko, D. (2007). Disparate letter and semantic category fluency deficits in autopsy-confirmed frontotemporal dementia and Alzheimer's disease. Neuropsychology, 21(1), 20. doi: 10.1037/08944105.21.1.20

Raymer, A. M., Foundas, A. L., Maher, L. M., Greenwald, M. L., Morris, M., Rothi, L. J. G., \& Heilman, K. M. (1997). Cognitive neuropsychological analysis and neuroanatomic correlates in a case of acute anomia. Brain and language, 58(1), 137-156. doi:10.1006/brln.1997.1786 
Raymer, A., \& Kohen, F. (2006). Word-retrieval treatment in aphasia: Effects of sentence context. Journal of rehabilitation research and development, 43(3), 367. doi: 10.1682/jrrd.2005.01.0028

Rayner, K., \& Reichle, E. D. (2010). Models of the reading process. Wiley Interdisciplinary Reviews. Cognitive Science, 1(6), 787-799. doi:10.1002/wcs.68

Recht, L. D., McCarthy, K., O'Donnell, B. F., Cohen, R., \& Drachman, D. A. (1989). Tumor- associated aphasia in left hemisphere primary brain tumors The importance of age and tumor grade. Neurology, 39(1), 48-48. doi: 10.1212/wnl.39.1.48

Ricard, D., Idbaih, A., Ducray, F., Lahutte, M., Hoang-Xuan, K., \& Delattre, J.-Y. (2012). Primary brain tumours in adults. Lancet, 379(9830), 1984-96. doi:10.1016/S0140$6736(11) 61346-9$

Ripamonti, E., Aggujaro, S., Molteni, F., Zonca, G., Frustaci, M., \& Luzzatti, C. (2014). The anatomical foundations of acquired reading disorders: A neuropsychological verification of the dual-route model of reading. Brain and Language, 134, 44-67. doi:10.1016/j.bandl.2014.04.001

Roberts, D. J., Woollams, A. M., Kim, E., Beeson, P. M., Rapcsak, S. Z., \& Lambon Ralph, M. A. (2012). Efficient Visual Object and Word Recognition Relies on High Spatial Frequency Coding in the Left Posterior Fusiform Gyrus: Evidence from a Case-Series of Patients with Ventral Occipito-Temporal Cortex Damage. Cerebral Cortex, 23(11), 2568-2580. doi:10.1093/cercor/bhs224

Robinson, G., Shallice, T., Bozzali, M., \& Cipolotti, L. (2010). Conceptual proposition selection and the LIFG: Neuropsychological evidence from a focal frontal group. Neuropsychologia, 48(6), 1652-1663. doi:10.1016/j.neuropsychologia.2010.02.010

Robinson, G., Shallice, T., Bozzali, M., \& Cipolotti, L. (2012). The differing roles of the frontal cortex in fluency tests. Brain, 135(7), 2202-2214. doi:10.1093/brain/aws142 
Robson, H., Grube, M., Lambon Ralph, M. A., Griffiths, T. D., \& Sage, K. (2013). Fundamental deficits of auditory perception in wernicke's aphasia. Cortex, 49(7), 1802-1822. Doi: 10.1016/j.cortex.2012.11.012

Robson, H., Sage, K., \& Lambon Ralph, M. A. (2012). Wernicke's aphasia reflects a combination of acoustic-phonological and semantic control deficits: a case-series comparison of Wernicke's aphasia, semantic dementia and semantic aphasia. Neuropsychologia, 50(2), 266-275. doi: 10.1016/j.neuropsychologia.2011.11.021

Roelofs, A. (1992). A spreading-activation theory of lemma retrieval in speaking. Cognition, 42(1-3), 107-142. doi:10.1016/0010-0277(92)90041-f

Roelofs, A. (2004). Comprehension-Based Versus Production-Internal Feedback in Planning Spoken Words: A Rejoinder to Rapp and Goldrick (2004). Psychological Review, 111(2), 579-580. doi:10.1037/0033-295X.111.2.579

Roeltgen, D. P., Sevush, S., \& Heilman, K. M. (1983). Phonological agraphia Writing by the lexical- semantic route. Neurology, 33(6), 755-755. doi:10.1212/WNL.33.6.755

Rogers, R. D., Sahakian, B. J., Hodges, J. R., Polkey, C. E., Kennard, C., \& Robbins, T. W. (1998). Dissociating executive mechanisms of task control following frontal lobe damage and Parkinson's disease. Brain : A Journal of Neurology, 121(5), 815-842. doi:10.1093/brain/121.5.815

Romani, C., \& Galluzzi, C. (2005). Effects of syllabic complexity in predicting accuracy of repetition and direction of errors in patients with articulatory and phonological difficulties. Cognitive Neuropsychology, 22(7), 817-850. doi:10.1080/02643290442000365

Romani, C., Olson, A., Semenza, C., \& Granà, A. (2002). Patterns of phonological errors as a function of a phonological versus an articulatory locus of impairment. Cortex, 38(4), 541-567. doi:10.1016/s0010-9452(08)70022-4 
Rorden, C., \& Karnath, H. O. (2004). Using human brain lesions to infer function: a relic from a past era in the fMRI age? Nature Reviews Neuroscience, 5(10), 812-819.doi: $10.1038 / \operatorname{nrn} 1521$

Rorden, C., Bonilha, L., Fridriksson, J., Bender, B., \& Karnath, H. O. (2012). Age-specific CT and MRI templates for spatial normalization. Neuroimage, 61(4), 957-965. doi:10.1016/j.neuroimage.2012.03.020

Rorden, C., Karnath, H. O., \& Bonilha, L. (2007). Improving lesion-symptom mapping. Journal of cognitive neuroscience, 19(7), 1081-1088. doi: 10.1162/jocn.2007.19.7.1081

Rossion, B., \& Pourtois, G. (2004). Revisiting Snodgrass and Vanderwart's object pictorial set: The role of surface detail in basic-level object recognition. PERCEPTIONLONDON-, 33(2), 217-236. doi: 10.1068/p5117

Rudrauf, D., Mehta, S., Bruss, J., Tranel, D., Damasio, H., \& Grabowski, T. J. (2008). Thresholding lesion overlap difference maps: Application to category-related naming and recognition deficits. Neuroimage, 41(3), 970-984. doi: 10.1016/j.neuroimage.2007.12.033

Ruml, W., Caramazza, A., Capasso, R., \& Miceli, G. (2005). Interactivity and continuity in normal and aphasic language production. Cognitive Neuropsychology, 22(2), 131-168. doi $=10.1080 / 02643290442000031$

Sacko, O., Lauwers-Cances, V., Brauge, D., Sesay, M., Brenner, A., \& Roux, F. E. (2011). Awake craniotomy vs surgery under general anesthesia for resection of supratentorial lesions. Neurosurgery, 68(5), 1192-1199. doi:10.1227/neu.0b013e31820c02a3

Sanai, N., Mirzadeh, Z., \& Berger, M. S. (2008). Functional outcome after language mapping for glioma resection. The New England Journal of Medicine, 358(1), 18-27. doi:10.1056/NEJMoa067819 
Scheibel, R. S., Meyers, C. A., \& Levin, V. A. (1996). Cognitive dysfunction following surgery for intracerebral glioma: influence of histopathology, lesion location, and treatment. Journal of neuro-oncology, 30(1), 61-69. doi:10.1007/bf00177444

Schmidt, M. (1996): Rey Auditory Verbal and Learning Test. A Handbook. Western Psychological Services, Los Angeles.

Schnur, T. T., Schwartz, M. F., Brecher, A. R., \& Hodgson, C. (2006). Semantic interference during blocked-cyclic naming: Evidence from aphasia. Journal of Memory and Language, 54(2), 199-227. doi:10.1016/j.jml.2005.10.002

Schnur, T. T., Schwartz, M. F., Kimberg, D. Y., Hirshorn, E., Coslett, H. B., \& ThompsonSchill, S. L. (2009). Localizing interference during naming: Convergent neuroimaging and neuropsychological evidence for the function of Broca's area. Proceedings of the National Academy of Sciences, 106(1), 322-327. doi: 10.1073/pnas.0805874106

Schriefers, H., Meyer, A. S., \& Levelt, W. J. M. (1990). Exploring the time course of lexical access in language production: Picture-word interference studies. Journal of Memory and Language, 29(1), 86-102. doi:10.1016/0749-596X(90)90011-N

Schwartz, M. F., \& Hodgson, C. (2002). A new multiword naming deficit: Evidence and interpretation. Cognitive Neuropsychology, 19(3), 263-288.

doi:10.1080/02643290143000187

Schwartz, M. F., Faseyitan, O., Kim, J., \& Coslett, H. B. (2012). The dorsal stream contribution to phonological retrieval in object naming. Brain, 135(12), 3799-3814. doi:10.1093/brain/aws300

Schwartz, M. F., Kimberg, D. Y., Walker, G. M., Faseyitan, O., Brecher, A., Dell, G. S., \& Coslett, H. B. (2009). Anterior temporal involvement in semantic word retrieval: voxel-based lesion-symptom mapping evidence from aphasia. Brain a Journal of Neurology, 132(Pt 12), 3411-3427. doi:10.1093/brain/awp284 
Schwartz, M., Dell, G., Martin, N., Gahl, S., \& Sobel, P. (2006). A case-series test of the interactive two-step model of lexical access: Evidence from picture naming. Journal of Memory and Language, 54(2), 228-264. doi:10.1016/j.jml.2005.10.001

Schwartz, S., \& Baldo, J. (2001). Distinct patterns of word retrieval in right and left frontal lobe patients: A multidimensional perspective. Neuropsychologia, 39(11), 1209-1217. doi:10.1016/s0028-3932(01)00053-7

Scott, R. M., \& Wilshire, C. E. (2010). Lexical competition for production in a case of nonfluent aphasia: Converging evidence from four different tasks. Cognitive neuropsychology, 27(6), 505-538. doi:10.1080/02643294.2011.598853

Seidenberg, M. S., \& McClelland, J. L. (1989). A distributed, developmental model of word recognition and naming. Psychological Review, 96(4), 523-568. doi:10.1037/0033295X.96.4.523

Shafi, N., \& Carozza, L (2011). Treating Cancer-Related Aphasia.

Shallice, T. (1981). Phonological agraphia and the lexical route in writing. Brain: a journal of neurology, 104(3), 413-429. doi:10.1093/brain/104.3.413

Shallice, T. (1988). From neuropsychology to mental structure. Cambridge University Press.

Shallice, T., \& Cooper, R. P. (2013). Is there a semantic system for abstract words?. Frontiers in human neuroscience, 7. doi: 10.3389/fnhum.2013.00175

Shallice, T., Mussoni, A., D’Agostino, S., \& Skrap, M. (2010). Right posterior cortical functions in a tumour patient series. Cortex, 46(9), 1178-1188. doi: 10.1016/j.cortex.2010.04.005

Shapiro, L. P., \& Levine, B. A. (1990). Verb processing during sentence comprehension in aphasia. Brain and Language, 38(1), 21-47. doi: 10.1016/0093-934X(90)90100-U 
Shapiro, L. P., Zurif, E., \& Grimshaw, J. (1987). Sentence processing and the mental representation of verbs. Cognition, 27(3), 219-246. doi: 10.1016/S00100277(87)80010-0

Shaywitz, B. A., Shaywitz, S. E., Pugh, K. R., Fulbright, R. K., Skudlarski, P., Mencl, W. E., Constable, R. T., et al. (2001). The functional neural architecture of components of attention in language-processing tasks. NeuroImage, 13(4), 601-612. doi: 10.1006/nimg.2000.0726

Sloan Berndt, R., Haendiges, A. N., Mitchum, C. C., \& Sandson, J. (1997b). Verb retrieval in aphasia. 2. Relationship to sentence processing. Brain and Language, 56(1), 107-137. doi:10.1006/brln.1997.1728

Sloan Berndt, R., Mitchum, C. C., Haendiges, A. N., \& Sandson, J. (1997a). Verb retrieval in aphasia. 1. Characterizing single word impairments. Brain and Language, 56(1), 68106. doi:10.1006/brln.1997.1727

Smith, A. (1982) Symbol Digit Modalities Test. Manual. Western Psychological Services, Los Angeles,

Speer, P., \& Wilshire, C. E. (2013). What's in a sentence? The crucial role of lexical content in sentence production in nonfluent aphasia. Cognitive neuropsychology, 30(7-8), 507-543. doi: 10.1080/02643294.2013.876398

Spitsyna, G., Warren, J. E., Scott, S. K., Turkheimer, F. E., \& Wise, R. J. S. (2006). Converging language streams in the human temporal lobe. The Journal of neuroscience, 26(28), 7328-7336. 10.1523/jneurosci.0559-06.2006

Spreen, O. (1998). A compendium of neuropsychological tests: Administration, norms, and commentary. Oxford University Press.

Squires, R. H. (1989). Vomiting as a Presenting Sign A Gastroenterologist's Perspective. Clinical pediatrics, 28(8), 351-354. doi:10.1177/000992288902800803 
Starreveld, P. A., \& La Heij, W. (1995). Semantic interference, orthographic facilitation, and their interaction in naming tasks. Journal Of Experimental Psychology. Learning Memory And Cognition, 21(3), 686-698. doi:10.1037/0278-7393.21.3.686

Starreveld, P. A., \& La Heij, W. (1996). Time-course analysis of semantic and orthographic context effects in picture naming. Journal Of Experimental Psychology. Learning Memory And Cognition, 22(4), 896-918. doi:10.1037/0278-7393.22.4.896

Starrfelt, R., Habekost, T., \& Leff, A. P. (2009). Too Little, Too Late: Reduced Visual Span and Speed Characterize Pure Alexia. Cerebral Cortex, 19(12), 2880-2890. doi:10.1093/cercor/bhp059

Stemberger, J. P. (1985). An interactive activation model of language production. Progress in the psychology of language, 1, 143-186.

Stroop, J. R. (1935). Studies of interference in serial verbal reactions. Journal of Experimental Psychology, 18(6), 643-662. doi:10.1037/h0054651

Strub, R. L., \& Gardner, H. (1974). The repetition defect in conduction aphasia: Mnestic or linguistic?. Brain and Language, 1(3), 241-255. doi:10.1016/0093-934x(74)90039-x

Stuss, D. T., Alexander, M. P., Hamer, L., Palumbo, C., Dempster, R., Binns, M., ... \& Izukawa, D. (1998). The effects of focal anterior and posterior brain lesions on verbal fluency. Journal of the International Neuropsychological Society, 4(03), 265-278. doi:10.1016/s0028-3932(97)00152-8

Swinburn, K., Porter, G., \& Howard, D. (2005) The comprehensive Aphasia Test. Hove, UK: Psychology Press.

Taft, M., \& Hambly, G. (1986). Exploring the Cohort Model of spoken word recognition. Cognition, 22(3), 259-282. doi:10.1016/0010-0277(86)90017-X 
Talacchi, A., Santini, B., Savazzi, S., \& Gerosa, M. (2011). Cognitive effects of tumour and surgical treatment in glioma patients. Journal of Neurooncology, 103(3), 541-549. doi:10.1007/s11060-010-0417-0

Tallal, P., \& Newcombe, F. (1978). Impairment of auditory perception and language comprehension in dysphasia. Brain and Language, 5(1), 13-34. doi:10.1016/0093$934 X(78) 90003-2$

Tandon, P., \& Mahapatra, A. K. (1993). Operations on gliomas involving speech centres (pp. 67-71). Springer Vienna.

Taphoorn, M. J. B., \& Klein, M. (2004). Review Cognitive deficits in adult patients with brain tumours. The Lancet, 31(0), 159-168. doi:10.1016/S1474-4422(04)00680-5

Taylor, B. V., Buckner, J. C., Cascino, T. L., O'Fallon, J. R., Schaefer, P. L., Dinapoli, R. P., \& Schomberg, P. (1998). Effects of radiation and chemotherapy on cognitive function in patients with high-grade glioma. Journal of clinical oncology, 16(6), 2195-2201. doi:10.1200/jco.2006.08.5605

Thomas, R., O’Connor, A. M., \& Ashley, S. (1995). Speech and language disorders in patients with high grade glioma and its influence on prognosis. Journal of Neurooncology, 23(3), 265-270. doi:10.1007/bf01059960

Thompson-Schill, S. L., \& Botvinick, M. M. (2006). Resolving conflict: A response to Martin and Cheng (2006). Psychonomic Bulletin \& Review, 13(3), 402-408. doi: $10.3758 / \mathrm{bf03} 193860$

Thompson-Schill, S. L., Bedny, M., \& Goldberg, R. F. (2005). The frontal lobes and the regulation of mental activity. Current opinion in neurobiology, 15(2), 219-224. doi: 10.1016/j.conb.2005.03.006 
Thompson-Schill, S. L., D’Esposito, M., \& Kan, I. P. (1999). Effects of repetition and competition on activity in left prefrontal cortex during word generation. Neuron, 23(3), 513-522. doi:10.1016/s0896-6273(00)80804-1

Thompson-Schill, S. L., D’Esposito, M., Aguirre, G. K., \& Farah, M. J. (1997). Role of left inferior prefrontal cortex in retrieval of semantic knowledge: A re-evaluation. Proceedings of the National Academy of Sciences of the United States of America, 94(26), 14792-14797. doi:10.1073/pnas.94.26.14792

Thompson, C. K., Lange, K. L., Schneider, S. L., \& Shapiro, L. P. (1997). Agrammatic and non-brain-damaged subjects' verb and verb argument structure production. Aphasiology, 11(4-5), 473-490.

Thompson, C. K., Shapiro, L. P., Li, L., \& Schendel, L. (1995). Analysis of verbs and verbargument structure: A method for quantification of aphasic language production. Clinical aphasiology, 23, 121-140. Retrieved from http://aphasiology.pitt.edu/archive/00000195/

Thompson, S. A., Patterson, K., \& Hodges, J. R. (2003). Left/right asymmetry of atrophy in semantic dementia Behavioral-cognitive implications. Neurology, 61(9), 1196-1203. doi:10.1212/01.wnl.0000091868.28557.b8

Trueswell, J. C., \& Kim, A. E. (1998). How to prune a graden path by nipping it in the bud: Fast priming of verb argument structure. Journal of Memory and Language, 39(1), 102-123. doi: 10.1006/jmla.1998.2565

Tsuchida, A., \& Fellows, L. K. (2012). Are core component processes of executive function dissociable within the frontal lobes? Evidence from humans with focal prefrontal damage. Cortex. doi:10.1016/j.cortex.2012.10.014 
Tucha, O., Smely, C., Preier, M., \& Lange, K. W. (2000). Cognitive deficits before treatment among patients with brain tumors. Neurosurgery, 47(2), 324-334.doi:

$10.1097 / 00006123-200008000-00011$

Tyler, L. K. (1984). The structure of the initial cohort: evidence from gating. Perception \& Psychophysics, 36(5), 417-427. doi:10.3758/BF03207496

Vallar, G., \& Baddeley, A. D. (1984). Fractionation of working memory: Neuropsychological evidence for a phonological short-term store. Journal of Verbal Learning and Verbal Behavior, 23(2), 151-161. doi:10.1016/s0022-5371(84)90104-х

Vitevitch, M. S., \& Luce, P. A. (1999). Probabilistic Phonotactics and Neighborhood Activation in Spoken Word Recognition. Journal of Memory and Language, 40(3), 374-408. doi:10.1006/jmla.1998.2618

Vitevitch, M. S., \& Luce, P. A. (2005). Increases in phonotactic probability facilitate spoken nonword repetition. Journal of Memory and Language, 52(2), 193-204. doi:10.1016/j.jml.2004.10.003

Wagner, S., Sebastian, A., Lieb, K., Tüscher, O., \& Tadić, A. (2014). A coordinate-based ALE functional MRI meta-analysis of brain activation during verbal fluency tasks in healthy control subjects. BMC Neuroscience, 15, 19. doi:10.1186/1471-2202-15-19

Walker, G. M., Schwartz, M. F., Kimberg, D. Y., Faseyitan, O., Brecher, A., Dell, G. S., \& Coslett, H. B. (2011). Support for anterior temporal involvement in semantic error production in aphasia: New evidence from VLSM. Brain and Language, 117(3), 110122. doi:10.1016/j.bandl.2010.09.008

Waters, G., Caplan, D., \& Hildebrandt, N. (1991). On the Structure of Verbal Short-term Memory and its Functional Role in Sentence Comprehension: Evidence from Neuropsychology. Cognitive Neuropsychology. doi:10.1080/02643299108253368 
Webster, J., \& Whitworth, A. (2012). Treating verbs in aphasia: exploring the impact of therapy at the single word and sentence levels. International Journal of Language Communication Disorders Royal College of Speech Language Therapists, 47(6), 61936. doi:10.1111/j.1460-6984.2012.00174.x

Wechsler, D. (1981) WAIS-R. Manual. Manual for Wechsler Adult Intelligence Scale-Revised. The Psychological Corporation, San Antonio.

White, J. G., Merrick, M., \& Harbison, J. J. M. (1969). Williams Scale for the Measurement of Memory: Test reliability and validity in a psychiatric population. British Journal of Social and Clinical Psychology, 8(2), 141-151. doi:10.1111/j.20448260.1969.tb00599.x

Whittle, I. R., Pringle, A. M., \& Taylor, R. (1998). Effects of resective surgery for left-sided intracranial tumours on language function: A prospective study. Lancet, 351(9108), 1014-1018. doi:10.1016/s0140-6736(97)08295-0

Williams, S. E., \& Canter, G. J. (1982). The influence of situational context on naming performance in aphasic syndromes. Brain and Language, 17(1), 92-106. doi:10.1016/0093-934x(82)90007-4

Wilshire, C. E. (2002). Where do aphasic phonological errors come from? Evidence from phoneme movement errors in picture naming. Aphasiology, 16(1-2), 169-197. doi: $10.1080 / 02687040143000528$

Wilshire, C. E. (2014) Cognitive Neuropsychology: Exploring the Mind Through Brain Dysfunction (in prep).

Wilshire, C. E. W., \& McCarthy, R. A. (2002). Evidence for a context-sensitive word retrieval disorder in a case of nonfluent aphasia. Cognitive Neuropsychology, 19(2), 165-186. doi:10.1080/02643290143000169 
Wilshire, C. E., \& McCarthy, R. A. (1996). Experimental investigations of an impairment in phonological encoding. Cognitive Neuropsychology, 13(7), 1059-1098. doi:10.1080/026432996381782

Wilshire, C. E., Keall, L. M., Stuart, E. J., \& O’Donnell, D. J. (2007). Exploring the dynamics of aphasic word production using the picture-word interference task: A case study. Neuropsychologia, 45(5), 939-953. doi:10.1016/j.band1.2005.07.057

Wu, C., Pu, S., Lin, Y., Wang, Y., Jiang, T., Xie, J., ... Wang, X. (2008). Fractionated resection on low grade gliomas involving Broca's area and insights to brain plasticity. Chinese Medical Journal, 121(20), 2026-2030. Retrieved from http://www.ncbi.nlm.nih.gov/pubmed/19080269

Zorzi, M., Houghton, G., \& Butterworth, B. (1998). The development of spelling-sound relationships in a model of phonological reading. Language and Cognitive Processes, 13(2-3), 337-371. doi:10.1080/016909698386555 